\title{
Monte Carlo Simulation for LINAC Standoff Interrogation of Nuclear Material
}

June 2007

Prepared by

Shaun Clarke

Marek Flaska

Thomas Miller

Vladimir Protopopescu

Sara Pozzi 


\title{
DOCUMENT AVAILABILITY
}

Reports produced after January 1, 1996, are generally available free via the U.S. Department of Energy (DOE) Information Bridge.

Web site http://www.osti.gov/bridge

Reports produced before January 1, 1996, may be purchased by members of the public from the following source.

National Technical Information Service

5285 Port Royal Road

Springfield, VA 22161

Telephone 703-605-6000 (1-800-553-6847)

TDD 703-487-4639

Fax 703-605-6900

E-mail info@ntis.gov

Web site http://www.ntis.gov/support/ordernowabout.htm

Reports are available to DOE employees, DOE contractors, Energy Technology Data Exchange (ETDE) representatives, and International Nuclear Information System (INIS) representatives from the following source.

Office of Scientific and Technical Information

P.O. Box 62

Oak Ridge, TN 37831

Telephone 865-576-8401

Fax 865-576-5728

E-mail reports@osti.gov

Web site http://www.osti.gov/contact.html

\begin{abstract}
This report was prepared as an account of work sponsored by an agency of the United States Government. Neither the United States government nor any agency thereof, nor any of their employees, makes any warranty, express or implied, or assumes any legal liability or responsibility for the accuracy, completeness, or usefulness of any information, apparatus, product, or process disclosed, or represents that its use would not infringe privately owned rights. Reference herein to any specific commercial product, process, or service by trade name, trademark, manufacturer, or otherwise, does not necessarily constitute or imply its endorsement, recommendation, or favoring by the United States Government or any agency thereof. The views and opinions of authors expressed herein do not necessarily state or reflect those of the United States Government or any agency thereof.
\end{abstract}


Nuclear Science and Technology Division

\title{
MONTE CARLO SIMULATION FOR LINAC STANDOFF INTERROGATION OF NUCLEAR MATERIAL
}

\author{
S.D. Clarke ${ }^{*}, 1$, M. Flaska ${ }^{2}$, T.M. Miller ${ }^{2}$, V. Protopopescu² ${ }^{2}$ and S.A. Pozzi ${ }^{2}$ \\ ${ }^{1}$ School of Nuclear Engineering, Purdue University, West Lafayette, IN 47907 \\ ${ }^{2}$ Oak Ridge National Laboratory, Oak Ridge, TN 37831
}

Date Published: June 2007

\author{
Prepared by \\ OAK RIDGE NATIONAL LABORATORY \\ P.O. Box 2008 \\ Oak Ridge, Tennessee 37831-6285 \\ managed by \\ UT-BATTELLE, LLC \\ for the \\ U.S. DEPARTMENT OF ENERGY \\ under contract DE-AC05-00OR22725
}

* On research assignment at Oak Ridge National Laboratory. 



\section{CONTENTS}

Page

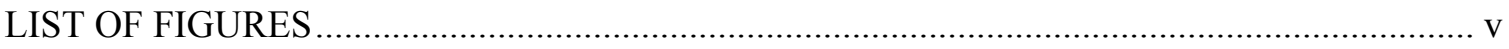

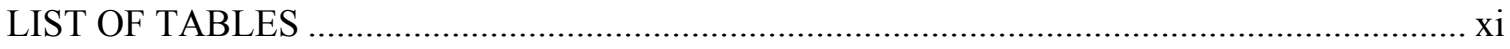

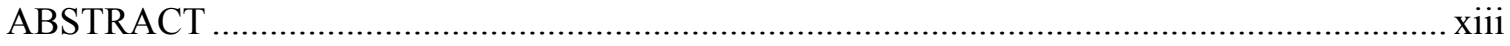

1. INTRODUCTION

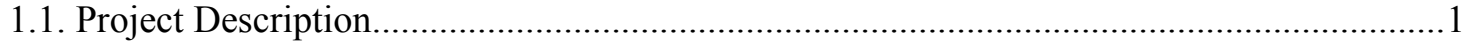

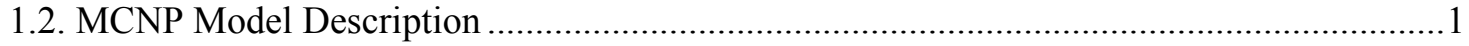

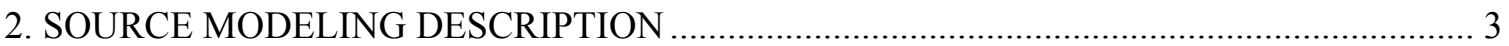

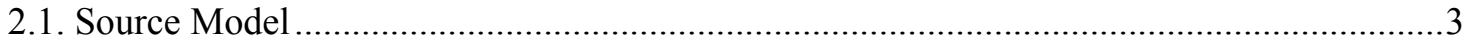

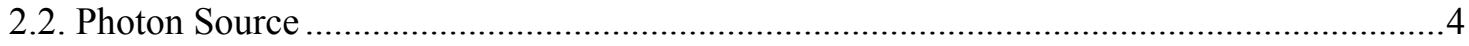

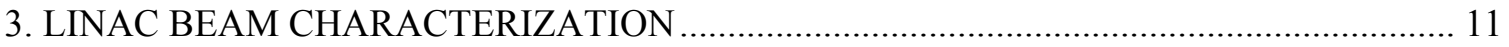

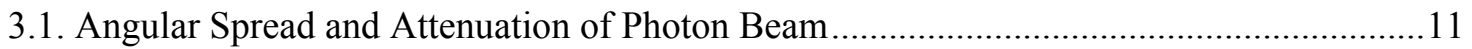

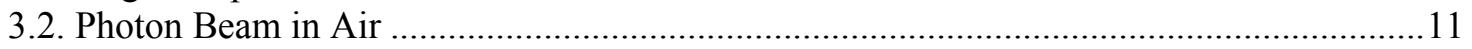

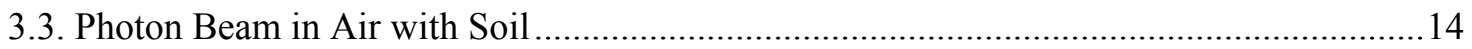

4. NEUTRON AND PHOTON FLUX MAPS IN MODEL GEOMETRY …............................... 18

5. TIME AND ENERGY DEPENDENCE OF NEUTRON AND PHOTON FLUX ................... 25

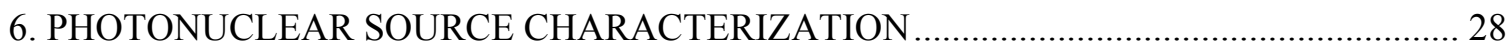

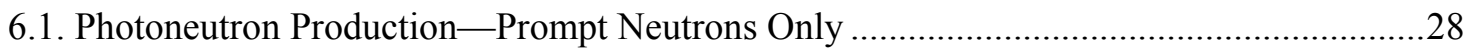

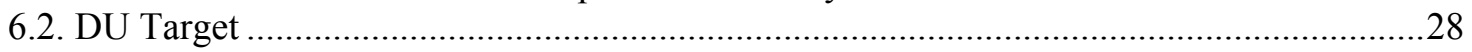

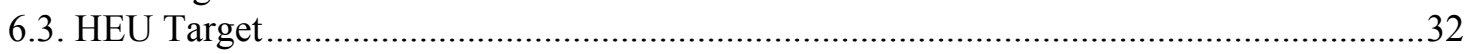

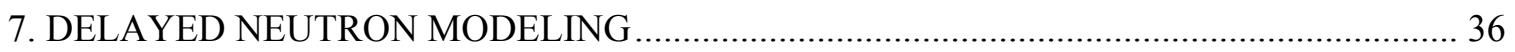

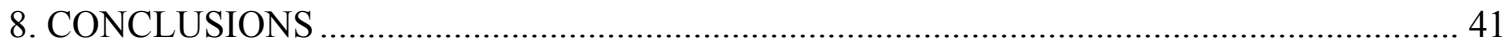

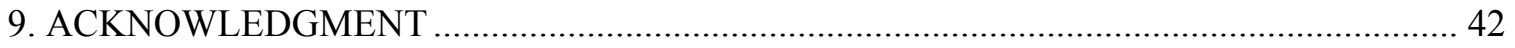

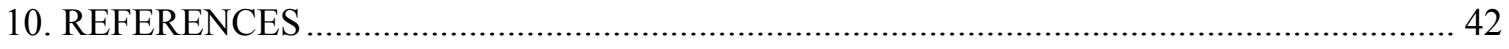

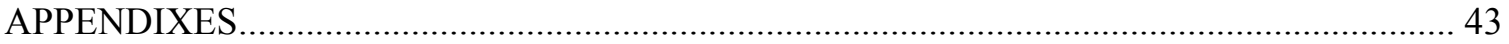

Appendix A. MCNPX Input File for Source Chracterization........................................ A-3

Appendix B. MCNPX Input Files for LINAC Beam Characterization ....................................

Appendix C. MCNPX Input Files for Neutron and Photon Flux Maps ....................................

Appendix D. Neutron and Photon Fluence Map Errors …................................................... D-3

Appendix E. MCNPX Input File for Time-Energy Dependence ........................................... E-3

Appendix F. MCNP-PoliMi Input Files for Photonuclear Sources …….............................. F-3

Appendix G. MCNPX Input File for the Delayed Neutron Source ..................................... G-3

Appendix H. Relative Errors on Delayed Neutron Fluence Maps .......................................... H-3 



\section{LIST OF FIGURES}

\section{$\begin{array}{lll}\text { Figure } & \text { Page }\end{array}$}

1. Illustration of the conceptual system including the LINAC and containment vehicle....... 2

2. MCNP model illustration showing the location of the $x-y$ plane tally regions.................. 3

3. Photon source current and flux vs. radial distance from beam axis. ............................... 8

4. Photon source current for the first radial region $(\mathrm{R}<0.15875 \mathrm{~cm})$ vs. energy............... 8

5. Photon source current and flux for the first radial region $(\mathrm{R}<0.15875 \mathrm{~cm})$ and first energy group $(4 \mathrm{MeV}<\mathrm{E}<4.25 \mathrm{MeV})$ vs. degrees from the beam axis. 9

6. Photon source current and flux for the first radial region $(\mathrm{R}<0.15875 \mathrm{~cm})$ and $64^{\text {th }}$ energy group $(19.75 \mathrm{MeV}<\mathrm{E}<20 \mathrm{MeV})$ vs. degrees from the beam axis

7. Photon source current and flux for the first radial region $(\mathrm{R}<0.15875 \mathrm{~cm})$ and first energy group ( $4 \mathrm{MeV}<\mathrm{E}<4.25 \mathrm{MeV}$ ) vs. degrees from the beam axis, 0 to 10 degrees.

8. Photon source current and flux for the first radial region $(\mathrm{R}<0.15875 \mathrm{~cm})$ and $64^{\text {th }}$ energy group $(19.75 \mathrm{MeV}<\mathrm{E}<20 \mathrm{MeV}$ ) vs. degrees from the beam axis, 0 to 10 degrees.

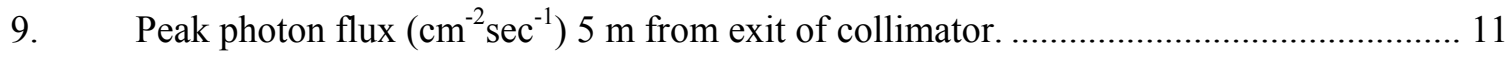

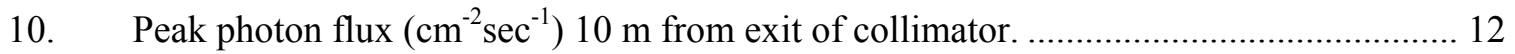

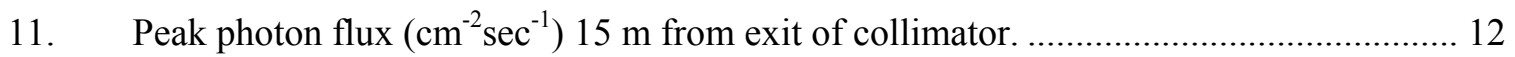

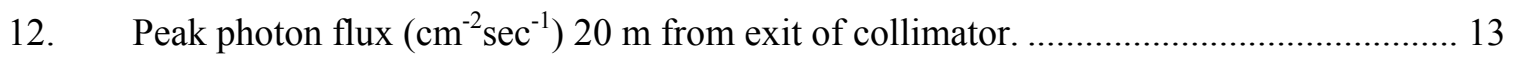

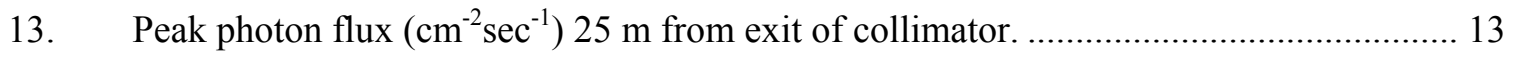

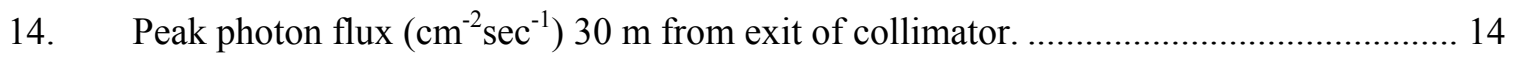

15. Peak photon flux $\left(\mathrm{cm}^{-2} \mathrm{sec}^{-1}\right)$ above the soil $5 \mathrm{~m}$ from exit of collimator. ....................... 15

16. Peak Photon flux $\left(\mathrm{cm}^{-2} \mathrm{sec}^{-1}\right)$ above the soil $10 \mathrm{~m}$ from exit of collimator...................... 16

17. Peak photon flux $\left(\mathrm{cm}^{-2} \mathrm{sec}^{-1}\right)$ above the soil $15 \mathrm{~m}$ from exit of collimator. ...................... 16

18. Peak Photon flux $\left(\mathrm{cm}^{-2} \mathrm{sec}^{-1}\right)$ above the soil $20 \mathrm{~m}$ from exit of collimator....................... 17

19. Peak photon flux $\left(\mathrm{cm}^{-2} \mathrm{sec}^{-1}\right)$ above the soil $25 \mathrm{~m}$ from exit of collimator. ..................... 17

20. Peak photon flux $\left(\mathrm{cm}^{-2} \mathrm{sec}^{-1}\right)$ above the soil $30 \mathrm{~m}$ from exit of collimator. ..................... 18 
21. Neutron fluence per source electron $\left(\mathrm{cm}^{-2}\right)$ over horizontal planes at an elevation of $24 \mathrm{~cm}$.

22. Neutron fluence per source electron $\left(\mathrm{cm}^{-2}\right)$ over horizontal planes at an elevation of $0 \mathrm{~cm}$.

23. Neutron fluence per source electron $\left(\mathrm{cm}^{-2}\right)$ over horizontal planes at an elevation of $-24 \mathrm{~cm}$

24. Neutron fluence per source electron $\left(\mathrm{cm}^{-2}\right)$ over horizontal planes at an elevation of $-33.62 \mathrm{~cm}$

25. Neutron fluence per source electron $\left(\mathrm{cm}^{-2}\right)$ over horizontal planes at an elevation of $-62 \mathrm{~cm}$.

26. Neutron fluence per source electron $\left(\mathrm{cm}^{-2}\right)$ over horizontal planes at an elevation of $-90.44 \mathrm{~cm}$.

27. Neutron fluence per source electron $\left(\mathrm{cm}^{-2}\right)$ over horizontal planes at an elevation of $-149 \mathrm{~cm}$.

28. Gamma ray fluence per source electron $\left(\mathrm{cm}^{-2}\right)$ over horizontal planes at an elevation of $0 \mathrm{~cm}$.

29. Gamma ray fluence per source electron $\left(\mathrm{cm}^{-2}\right)$ over horizontal planes at an elevation of $-20 \mathrm{~cm}$.

30. Gamma ray fluence per source electron $\left(\mathrm{cm}^{-2}\right)$ over horizontal planes at an elevation of $-37.62 \mathrm{~cm}$

31. Gamma ray fluence per source electron $\left(\mathrm{cm}^{-2}\right)$ over horizontal planes at an elevation of $-146 \mathrm{~cm}$.

32. Number of neutrons crossing the vehicle wall located vertically along the beamline for times between 20 and $50 \mathrm{~ns}$.

33. Number of neutrons crossing the vehicle wall located vertically along the beam line between 50 and $80 \mathrm{~ns}$.

34. Number of neutrons crossing the vehicle wall located vertically along the beam line between 100 and $400 \mathrm{~ns}$.

35. Number of neutrons crossing the vehicle wall located vertically, perpendicular to the beamline in the forward direction, for times between 3.25 and $10 \mathrm{~ns}$.

36. Number of neutrons crossing the vehicle wall located vertically, perpendicular to the beamline in the forward direction, for times between 10 and $40 \mathrm{~ns}$. 
37. Number of neutrons crossing the vehicle wall located vertically, perpendicular to the beamline in the forward direction, for times between 40 and $70 \mathrm{~ns}$.

38. Depleted uranium prompt photoneutron flux $\left(\mathrm{cm}^{-2} \mathrm{sec}^{-1}\right) 5 \mathrm{~m}$ from exit of the collimator (at target).

39. Depleted uranium prompt photoneutron flux $\left(\mathrm{cm}^{-2} \mathrm{sec}^{-1}\right) 3 \mathrm{~m}$ from exit of the collimator.

40. Depleted uranium prompt photoneutron flux $\left(\mathrm{cm}^{-2} \mathrm{sec}^{-1}\right) 6.5 \mathrm{~cm}$ from exit of the collimator.

41. Depleted uranium prompt photoneutron flux $\left(\mathrm{cm}^{-2} \mathrm{sec}^{-1}\right)$ on the side of the vehicle $4 \mathrm{~cm}$ downstream from the converter..

42. Depleted uranium prompt photoneutron flux $\left(\mathrm{cm}^{-2} \mathrm{sec}^{-1}\right)$ on the side of the vehicle $56 \mathrm{~cm}$ upstream from the converter..

43. Depleted uranium prompt photoneutron flux $\left(\mathrm{cm}^{-2} \mathrm{sec}^{-1}\right)$ on the side of the vehicle $96 \mathrm{~cm}$ upstream from the converter..

44. Highly enriched uranium prompt photoneutron flux $\left(\mathrm{cm}^{-2} \mathrm{sec}^{-1}\right) 5 \mathrm{~m}$ from exit of the collimator (at target).

45. Highly enriched uranium prompt photoneutron flux $\left(\mathrm{cm}^{-2} \mathrm{sec}^{-1}\right) 3 \mathrm{~m}$ from exit of the collimator.

46. Highly enriched uranium prompt photoneutron flux $\left(\mathrm{cm}^{-2} \mathrm{sec}^{-1}\right) 6.5 \mathrm{~cm}$ from exit of the collimator.

47. Highly enriched uranium prompt photoneutron flux $\left(\mathrm{cm}^{-2} \mathrm{sec}^{-1}\right)$ along the side of the vehicle $4 \mathrm{~cm}$ downstream from the converter.

48. Highly enriched uranium prompt photoneutron flux $\left(\mathrm{cm}^{-2} \mathrm{sec}^{-1}\right)$ along the side of the vehicle $56 \mathrm{~cm}$ upstream from the converter (flux on the other side of the vehicle is symmetric)....

49. Highly enriched uranium prompt photoneutron flux $\left(\mathrm{cm}^{-2} \mathrm{sec}^{-1}\right)$ along the side of the vehicle $96 \mathrm{~cm}$ upstream from the converter (flux on the other side of the vehicle is symmetric)....

50. Maxwellian energy spectrum (400 keV) used to approximate the delayed neutron source. The flux is tallied on the surface of the target per source delayed neutron; three-sigma error bars are shown.

51. Delayed neutron fluence per source delayed neutron $\left(\mathrm{cm}^{-2}\right)$ in the $x$ - $z$ plane centered about $\mathrm{y}=0 \mathrm{~cm}$; this is the center-plane of the geometry. 
52. Delayed neutron fluence per source delayed neutron $\left(\mathrm{cm}^{-2}\right)$ in the $x$-z plane centered

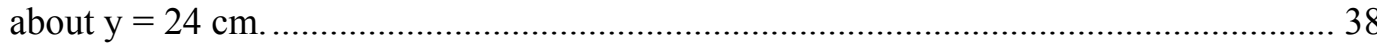

53. Delayed neutron fluence per source delayed neutron $\left(\mathrm{cm}^{-2}\right)$ in the $x$ - $z$ plane centered about $\mathrm{y}=-24 \mathrm{~cm}$

54. Delayed neutron fluence per source delayed neutron $\left(\mathrm{cm}^{-2}\right)$ in the $x-z$ plane centered about $\mathrm{y}=-33.62 \mathrm{~cm}$.

55. Delayed neutron fluence per source delayed neutron $\left(\mathrm{cm}^{-2}\right)$ in the $x-z$ plane centered about $\mathrm{y}=-62 \mathrm{~cm}$

56. Delayed neutron fluence per source delayed neutron $\left(\mathrm{cm}^{-2}\right)$ in the $x-z$ plane centered about $\mathrm{y}=-90.44 \mathrm{~cm}$.

57. Delayed neutron fluence per source delayed neutron $\left(\mathrm{cm}^{-2}\right)$ in the $x$ - $z$ plane centered about $\mathrm{y}=-149 \mathrm{~cm}$.

58. Delayed neutron fluence in the $x$-z-plane centered about $\mathrm{y}=0$-cm scaled to reflect production per source electron.

D-1. Relative errors for the neutron fluence map shown in Figure 18............................... D-3

D-2. Relative errors for the neutron fluence map shown in Figure 19.............................. D-3

D-3. Relative errors for the neutron fluence map shown in Figure 20 ............................. D-4

D-4. Relative errors for the neutron fluence map shown in Figure 21 .............................. D-4

D-5. Relative errors for the neutron fluence map shown in Figure 22.............................. D-5

D-6. Relative errors for the neutron fluence map shown in Figure 23............................. D-5

D-7. Relative errors for the neutron fluence map shown in Figure 24 ............................. D-6

D-8. Relative errors for the gamma ray fluence map shown in Figure 25........................ D-6

D-9. Relative errors for the gamma ray fluence map shown in Figure 26.......................... D-7

D-10. Relative errors for the gamma ray fluence map shown in Figure 27.......................... D-7

D-11. Relative errors for the gamma ray fluence map shown in Figure 28......................... D-8

H-1. Relative errors for the delayed neutron fluence map shown in Figure 48..................... H-3

H-2. Relative errors for the delayed neutron fluence map shown in Figure 49.................... H-3

H-3. Relative errors for the delayed neutron fluence map shown in Figure 50..................... H-4 
H-4. Relative errors for the delayed neutron fluence map shown in Figure 51 ..................... H-4

H-5. Relative errors for the delayed neutron fluence map shown in Figure 52 .................... H-5

H-6. Relative errors for the delayed neutron fluence map shown in Figure 53 ..................... H-5

H-7. Relative errors for the delayed neutron fluence map shown in Figure 54 ..................... H-6 



\section{LIST OF TABLES}

Table

Page

1. First comparison of photon source to electron source .................................................. 6

2. Second comparison of photon source to electron source. ............................................. 6

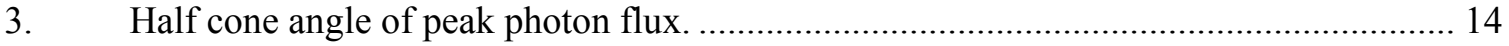





\begin{abstract}
The development of new techniques for the interrogation of shielded nuclear materials relies on the use of Monte Carlo codes to accurately simulate the entire system, including the interrogation source, the fissile target and the detection environment. The objective of this modeling effort is to develop analysis tools and methods - based on a relevant scenario - which may be applied to the design of future systems for active interrogation at a standoff. For the specific scenario considered here, the analysis will focus on providing the information needed to determine the type and optimum position of the detectors. This report describes the results of simulations for a detection system employing gamma rays to interrogate fissile and nonfissile targets. The simulations were performed using specialized versions of the codes MCNPX and MCNP-PoliMi. Both prompt neutron and gamma ray and delayed neutron fluxes have been mapped in three dimensions. The time dependence of the prompt neutrons in the system has also been characterized

For this particular scenario, the flux maps generated with the Monte Carlo model indicate that the detectors should be placed approximately $50 \mathrm{~cm}$ behind the exit of the accelerator, $40 \mathrm{~cm}$ away from the vehicle, and $150 \mathrm{~cm}$ above the ground. This position minimizes the number of neutrons coming from the accelerator structure and also receives the maximum flux of prompt neutrons coming from the source. The lead shielding around the accelerator minimizes the gamma-ray background from the accelerator in this area. The number of delayed neutrons emitted from the target is approximately seven orders of magnitude less than the prompt neutrons emitted from the system. Therefore, in order to possibly detect the delayed neutrons, the detectors should be active only after all prompt neutrons have scattered out of the system. Preliminary results have shown this time to be greater than $5 \mu$ s after the accelerator pulse.

This type of system is illustrative of a host of real-world scenarios of interest to nonproliferation and homeland security. Due to the multistep procedure of the MCNPX/MCNP-PoliMi code system, the analysis of somewhat modular - meaning that changing details such as the detector type, position, or surroundings does not require a re-calculation of the source-target interactions. This feature allows for efficient parametric analysis of numerous system parameters without recomputing the constant source-target behavior. Such efficient analysis mechanisms could prove invaluable in the design and future deployment of an active interrogation detection system.
\end{abstract}





\section{INTRODUCTION}

\subsection{Project Description}

Active measurements based on the use of photon sources from accelerators to induce fission show promise in many applications in nuclear nonproliferation, international safeguards, nuclear material control and accountability, national security, and counter-terrorism programs. A complete active interrogation system may be understood as a series of the following steps:

1. A linear electron accelerator (LINAC) produces an electron beam.

2. The electron beam strikes a target producing photons. Secondary neutrons are also produced.

3. The photons are collimated into a beam which strikes the target to be interrogated producing neutrons and gamma rays.

4. This radiation is transported to the detectors.

5. A specific detector response is produced through interactions within the detector material.

6. The timing and energy of the detected particles may be used to discriminate radiation created in the target and other sources of background radiation.

This report describes the results for steps 1 through 3 performed with specialized versions of the codes MCNP-X [1] and MCNP-PoliMi [2]. The objective of these simulations was to study the prompt and delayed response of unshielded fissile material to interrogation with a portable linear electron accelerator (LINAC) source of high energy photons. The LINAC source is assumed to be transportable on a small vehicle, and photon collimation is obtained with a portable lead collimator. This type of system is illustrative of a host of real-world scenarios of interest to nonproliferation and homeland security, namely active interrogation at a standoff.

Numerous simulations were performed to compute the neutron and photon flux inside the vehicle, fissile material, and surroundings. Initial simulations were performed to determine the bremsstrahlung photon source in energy and angular distribution using electrons as source particles. Subsequent simulations were used to determine the distribution and type of photonuclear events occurring in the target. Prompt, or "beam on," neutron and photon fluxes and delayed, or "beam off," neutron fluxes were then simulated for the system under consideration. These results are useful for the selection and/or development of a neutron and gamma ray detector system that could be used to detect prompt and delayed radiation emissions following the interrogation of a fissile sample.

The analysis has been carried out in four primary steps: (1) LINAC source modeling and characterization, (2) steady-state mapping of the neutron and photon fields, (3) time-dependant behavior of the neutron and photon fields, and (4) evaluation of the delayed neutron source.

\subsection{MCNP Model Description}

These analyses were performed using the MCNPX/MCNP-PoliMi code system [2]. Figure 1 shows the conceptual system with the LINAC being contained inside a vehicle and the detectors mounted on the sides (hypothetically). The objective of this modeling effort is to develop analysis tools and methods based on a relevant scenario which may be applied to the design of future systems for active interrogation at a standoff. For the specific scenario considered here, the 
analysis will focus on providing the information needed to determine the type, and optimum position of the detectors.

Several assumptions were necessary to create a MCNP model of this system. The walls of the vehicle were assumed to be 0.63 -cm-thick stainless steel. The LINAC was assumed to occupy an air-filled volume of $150 \times 50 \times 50 \mathrm{~cm}$. The shielding around the LINAC was $7.62 \mathrm{~cm}$ of natural lead on all sides except for the front, which was $15.24 \mathrm{~cm}$. The collimator opening was conical in shape with its diameter decreasing from $2.54 \mathrm{~cm}$ to $1.905 \mathrm{~cm}$. All air was modeled at a relative humidity of $45 \%$ and the soil contained $20 \%$ water. The target material was a $5-\mathrm{kg}(3.9979-\mathrm{cm}$ radius) sphere of depleted uranium $\left(100 \%{ }^{238} \mathrm{U}\right)$ placed $5 \mathrm{~m}$ from the LINAC along the beamline, $1.5 \mathrm{~m}$ above the ground. This target size and shape is the design standard used in active interrogation systems.

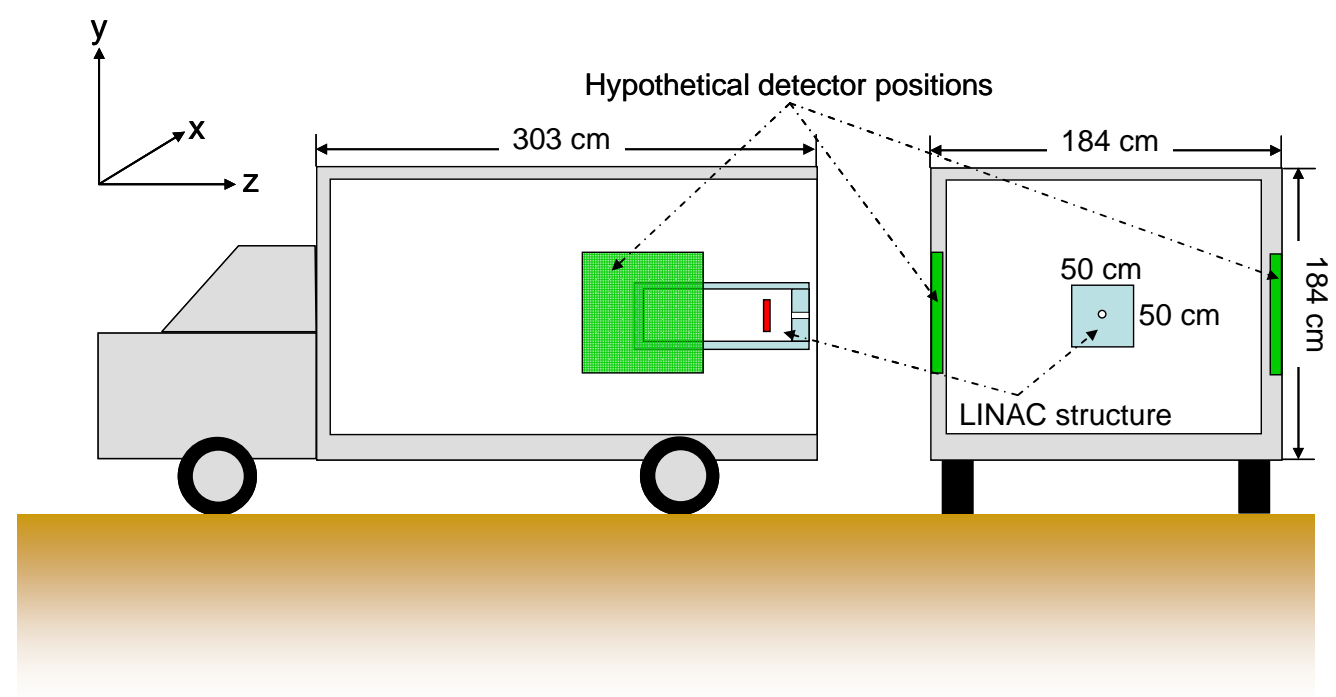

Figure 1. Illustration of the conceptual system including the LINAC and containment vehicle. The detectors shown were not modeled and are for illustrative purposes only.

The model was used to calculate flux maps in the $x-y$ and $x$-z planes at various $z$ and $y$ locations, respectively. In order to compute the fluxes in the $x-y$ plane, a series of tally volumes was added to the model; each of the planes shown extends $3 \mathrm{~m}$ in both the $\pm x$ and $\pm z$ directions. A fixed grid of $10 \times 10 \times 10 \mathrm{~cm}^{3}$ was used to compute the volume-averaged flux in the $x-y$ plane. The space behind the LINAC was the primary region of interest in the model — based on both physics and geometric considerations - and is the most likely location for the detectors. Therefore, a higher concentration of tally volumes was applied in this area. Figure 2 shows these regions. 


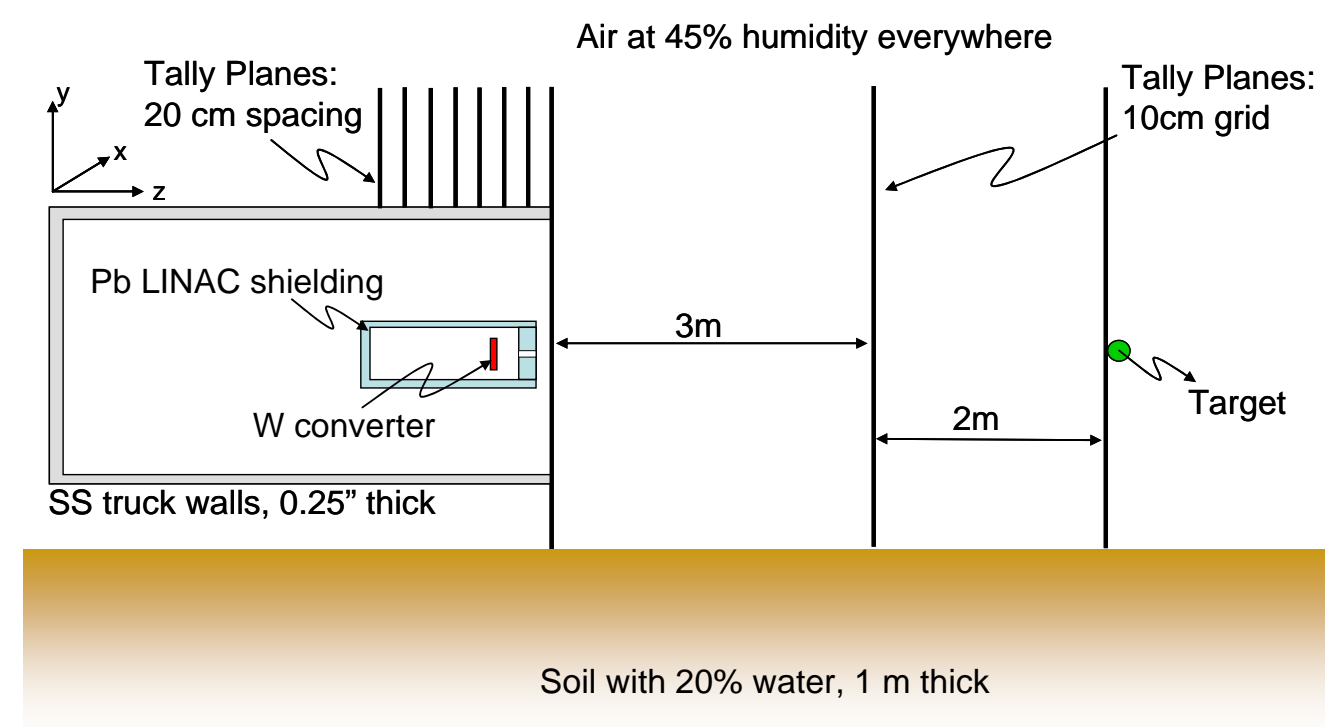

Figure 2. MCNP model illustration showing the location of the $x-y$ plane tally regions.

The flux in the $x-z$ plane was computed using a $2 \times 2 \times 2-\mathrm{cm}^{3}$ grid at several positions of interest in the $y$-direction:

1. below the vehicle $(y=-149 \mathrm{~cm})$

2. just inside the vehicle $(y=-90.44 \mathrm{~cm})$

3. half-way between the shielding and vehicle wall $(y=-62 \mathrm{~cm})$

4. just outside the LINAC shielding $(y=-33.62)$

5. just inside the LINAC shielding $(y= \pm 24 \mathrm{~cm})$

6. the geometric mid-plane $(y=0 \mathrm{~cm})$

The remainder of this report is organized as follows: Section 2 describes the specification of a photon source generated by the LINAC. Section 3 presents the results from the attenuation and angular spread of the photon beam. Section 4 describes the neutron and photon fluxes in the model geometry. Section 5 describes the time and energy-dependence of the neutron flux distributions. Section 6 presents the modeling of photonuclear events in the target material. Section 7 describes the delayed neutron production. Finally, Section 8 summarizes our results to date and indicates possible research directions. Appendixes A-H contain input files and map error data.

\section{SOURCE MODELING DESCRIPTION}

\subsection{Source Model}

The proposed source of this active interrogation system is a compact portable LINAC. The characteristics of this accelerator were assumed:

- Electron energy of $25 \mathrm{MeV} \pm 5 \%$

- Repetition rate of $120 \mathrm{~Hz}$

- Pulse time width between 500 and $2500 \mathrm{~ns}$ 
- Charge per pulse of $25 \mathrm{nC}$

These characteristics result in approximately $1.872 \mathrm{e} 13$ electrons per second:

$$
\frac{25 n C}{\text { pulse }} \cdot \frac{120 \text { pulse }}{\mathrm{s}} \cdot \frac{\mathrm{C}}{10^{9} \mathrm{nC}} \cdot \frac{\text { electron }}{1.602 \cdot 10^{-19} \mathrm{C}}=1.872 e 13 \text { electrons } / \mathrm{s}
$$

In order to make the source model more realistic, variations in three of the beam parameters were simulated using typical values seen at electron accelerator facilities. First, the electron energy was sampled with a variation of plus-or-minus 5\%. Secondly, the beam direction was sampled with a variation of $\pm 1^{\circ}$ with respect to the beam axis. Finally, the electron position was sampled inside of a $0.25-\mathrm{cm}$ radius around the beam axis. In all simulations utilizing an electron source (input file shown in Appendix A), these three parameters were sampled uniformly between the maximum and minimum allowable values, the particles always start on the upstream side of the tungsten converter, and the beam axis passes through the radial center of the opening in the beam collimator as shown in Figure 1.

\subsection{Photon Source}

Beginning the simulations with a bremsstrahlung photon source directly, instead of an electron source, significantly reduces the computational time required for each calculation. Charged particle transport in Monte Carlo is computationally inefficient due to the continuous slowing down model that is applied; transporting only photons and electrons is an inherently simpler problem. By performing one coupled neutron-electron-photon simulation of the bremsstrahlung spectrum exiting the electron accelerator, a position-, energy-, and angular-dependent photon spectrum may be generated. This source - which is valid only for the specific set of LINAC properties applied here - can then be used directly as a source in subsequent calculations of the photonuclear spectrum in the target.

There are two possible methods to calculate the desired bremsstrahlung spectrum. The most accurate method is to have MCNPX write a surface crossing file that can be read as a source by other MCNPX calculations. The second is to create a surface tally in MCNPX that has a sufficiently large number of energy, angular, and spatial bins to accurately represent the photons leaking from the accelerator shielding. The second method was chosen because the first requires writing a binary file, which can become very large and also be platform- and/or compilerdependent.

The surface tally that was created in MCNPX tallied all photons crossing the plane on the outside surface of the accelerator shielding. This surface is the closest to the target area and perpendicular to the beam axis as shown in Figure 1. The position, energy, and angular segmentation of the tally are listed below:

- The surface was divided into 27 radial rings centered on the beam axis. The outside radii of each ring (the first being a disc not a ring) in centimeters are $0.47625,0.9525$ (this corresponds to the radius at the exit of the beam collimator), 1.27 (this corresponds to the radius at the entrance of the beam collimator), 2.27 to 5.27 by steps of $1 ; 7.27,9.27$ to 18.27 by steps of $3 ; 20.27,22.86$ to 46.86 by steps of 2 , and 50 . The area outside of the $50-\mathrm{cm}$ radius was tallied as one large bin. 
- The energy range was divided into 104 equally spaced bins from $4 \mathrm{MeV}$ to $30 \mathrm{MeV}$ in steps of $0.25 \mathrm{MeV}$.

- The angles were divided into 45 bins, where the direction parallel to the beam axis moving from the accelerator towards the target was the $0^{\circ}$ angle. The upper angles of these bins in degrees are: 0.1 to 3 in steps of $0.1,5,7.5 ; 10$ to 40 in steps of $5 ; 50$ to 90 in steps of 10; and 180 .

In order to test the equivalence of this position, energy, and angular-dependent photon source, tally results in the target were compared to those obtained directly using the electron source. The current of photons entering a $5-\mathrm{cm}$ radius spherical target and with zero importance was tallied in the MCNPX calculation. The importance was set to zero inside the sphere to be certain that only photons entering the sphere were counted. The photon current was calculated with the sphere in three different locations along the beam axis: $1 \mathrm{~m}, 5 \mathrm{~m}$, and $10 \mathrm{~m}$ from the beam collimator. Table 1 shows a comparison of the photon current on this sphere for the electron and photon sources at these locations. 
Table 1. First comparison of photon source to electron source

\begin{tabular}{|c|c|c|c||}
\hline $\begin{array}{c}\text { Distance of sphere } \\
\text { from collimator }(\mathrm{m})\end{array}$ & $\begin{array}{c}\text { Photon current with } \\
\text { electron source }(\gamma / \mathrm{s})\end{array}$ & $\begin{array}{c}\text { Photon current with } \\
\text { photon source }(\gamma / \mathrm{s})\end{array}$ & $\begin{array}{c}\text { Ratio of photon source } \\
\text { to electron source }\end{array}$ \\
\hline \hline 1 & $1.2410 \mathrm{e} 11$ & $1.2392 \mathrm{e} 11$ & 0.9985 \\
5 & $1.9552 \mathrm{e} 10$ & $2.3082 \mathrm{e} 10$ & 1.1805 \\
10 & $5.2819 \mathrm{e} 9$ & $5.7262 \mathrm{e} 9$ & 1.0841 \\
\hline
\end{tabular}

The results in Table 1 are encouraging; however, it is unexpected that the comparison at $10 \mathrm{~m}$ is better than at $5 \mathrm{~m}$. One possibility is that the current at $10 \mathrm{~m}$, relative to the current at $5 \mathrm{~m}$, consists of photons closer to the beam axis with very small scattering angles. The areas closest to the beam axis and the smallest scattering angles have been modeled with the finest detail. Thus, if the current at $5 \mathrm{~m}$ is dominated by photons in coarse position or angular bins, then a larger difference is expected. With this in mind, the tally bins were modified as follows:

- The surface was divided into 45 rings where the outside radius of each in centimeters is 0.15875 to 1.27 in steps of $0.15875 ; 1.77$ to 7.27 in steps of $0.5 ; 8.27$ to 14.27 in steps of $1 ; 16.27$ to 48.27 in steps of 2 ; and 50. Again, the area outside of the $50-\mathrm{cm}$ radius was tallied as one large bin.

- The energy bins were unchanged.

- The angles were divided into 112 bins where the upper angle of each bin in degrees is 0.1 to 10 in steps of $0.1 ; 15$ to 40 in steps of $5 ; 50$ to 90 in steps of 10 ; and 180 .

Unfortunately, this increase in the spatial and angular resolution had no significant effect on the comparison of the photon current tallies. Table 2 shows the comparison of the photon current tallies with the electron source and this new photon source.

Table 2. Second comparison of photon source to electron source

\begin{tabular}{|c|c|c|c||}
\hline $\begin{array}{c}\text { Distance of sphere } \\
\text { from collimator }(\mathrm{m})\end{array}$ & $\begin{array}{c}\text { Photon current with } \\
\text { electron source }(\gamma / \mathrm{s})\end{array}$ & $\begin{array}{c}\text { Photon current with } \\
\text { photon source }(\gamma / \mathrm{s})\end{array}$ & $\begin{array}{c}\text { Ratio of photon source } \\
\text { to electron source }\end{array}$ \\
\hline \hline 1 & $1.2410 \mathrm{e} 11$ & $1.2399 \mathrm{e} 11$ & 0.9991 \\
5 & $1.9552 \mathrm{e} 10$ & $2.3044 \mathrm{e} 10$ & 1.1786 \\
10 & $5.2819 \mathrm{e} 9$ & $5.7319 \mathrm{e} 9$ & 1.0852 \\
\hline
\end{tabular}

At this point, there are two options for improving the agreement of the photons source: either the MCNPX surface crossing file can be used or even finer resolution is needed in the photon current tally bins. Finer resolution is most likely not needed for the energy or spatial bins, but it may be needed in the angular bins. However, the exact region of the angular distribution requiring the finer resolution is not known. Three possibilities exists.

1. The bin size above $10^{\circ}$ needs to be decreased.

2. The bin size below $10^{\circ}$ needs to be decreased.

3. Both regions require finer resolution.

In the future, each of these possibilities should be considered in detail. However, considering that the majority of the photons crossing the tally surface pass through the collimator opening, it seems likely that the angular resolution needs to be refined for angles less than $10^{\circ}$. Finer resolution is probably only needed for angles less than a few degrees. It is important to note that 
at some point, the resolution can become so fine that it becomes difficult to obtain convergence of the statistical tally solution in MCNPX.

The following six figures (Figures 3-8) display the photon current tally results used to construct a picture of what the photon source looks like. Figure 3 shows the current and flux as a function of radius from the beam axis. The blue curve in Figure 3 is the current in each radial zone and the red curve is the flux or the current normalized to the area of each radial zone. Since the size of the radial zones varies, nonphysical increases in the current are present. However, the flux, which has been normalized to the area of each radial zone, does not show any of these nonphysical increases. The increase in the flux in Figure 3 around $32 \mathrm{~cm}$ occurs because this is the edge of the LINAC shielding. Figure 4 shows the current in the inner-most spatial tally region (circle) as a function of energy. The current is only shown in Figure 4 because all the energy tally bins were $0.25-\mathrm{MeV}$ wide. Figure 5 shows the current in the inner-most spatial tally region and energy group from $4 \mathrm{MeV}$ to $4.25 \mathrm{MeV}$ as a function of angle. Figure 6 shows the current as a function of angle for this same inner-most spatial tally region, but for the energy group from $19.75 \mathrm{MeV}$ to $20 \mathrm{MeV}$. Like Figure 3, Figure 5 and Figure 6 show the current with a blue curve and the flux or the current normalized to the width of the angular bins with a red curve. Figure 7 and Figure 8 are the same as Figure 5 and Figure 6, respectively, but show more detail from 0 to $10^{\circ}$ (in Figure 7) and from 0 to $1^{\circ}$ (in Figure 8). However, note that in Figure 7 and Figure 8 only the flux is shown along with the one-sigma standard deviation. In Figure 5 and Figure 6 the peak flux is less than $1^{\circ}$. This peak is due to the photons created in the tungsten converter that pass directly through the collimator opening. In Figure 5, the number of photons at broader angles increases due to photon build up in the LINAC shielding. In Figure 6, there is no photon build up because the energy is close to the electron beam energy (19.75 to $20 \mathrm{MeV})$. In Figure 7, the flux minimum between about 0.7 and $4.2^{\circ}$ is suffering from poor statistical convergence. In fact, some parts of the curves are missing (i.e., there were no tallies in that angular bin), and in some cases, the minus-one-sigma curve only is missing, which is due to a relative error of 1.0 . 


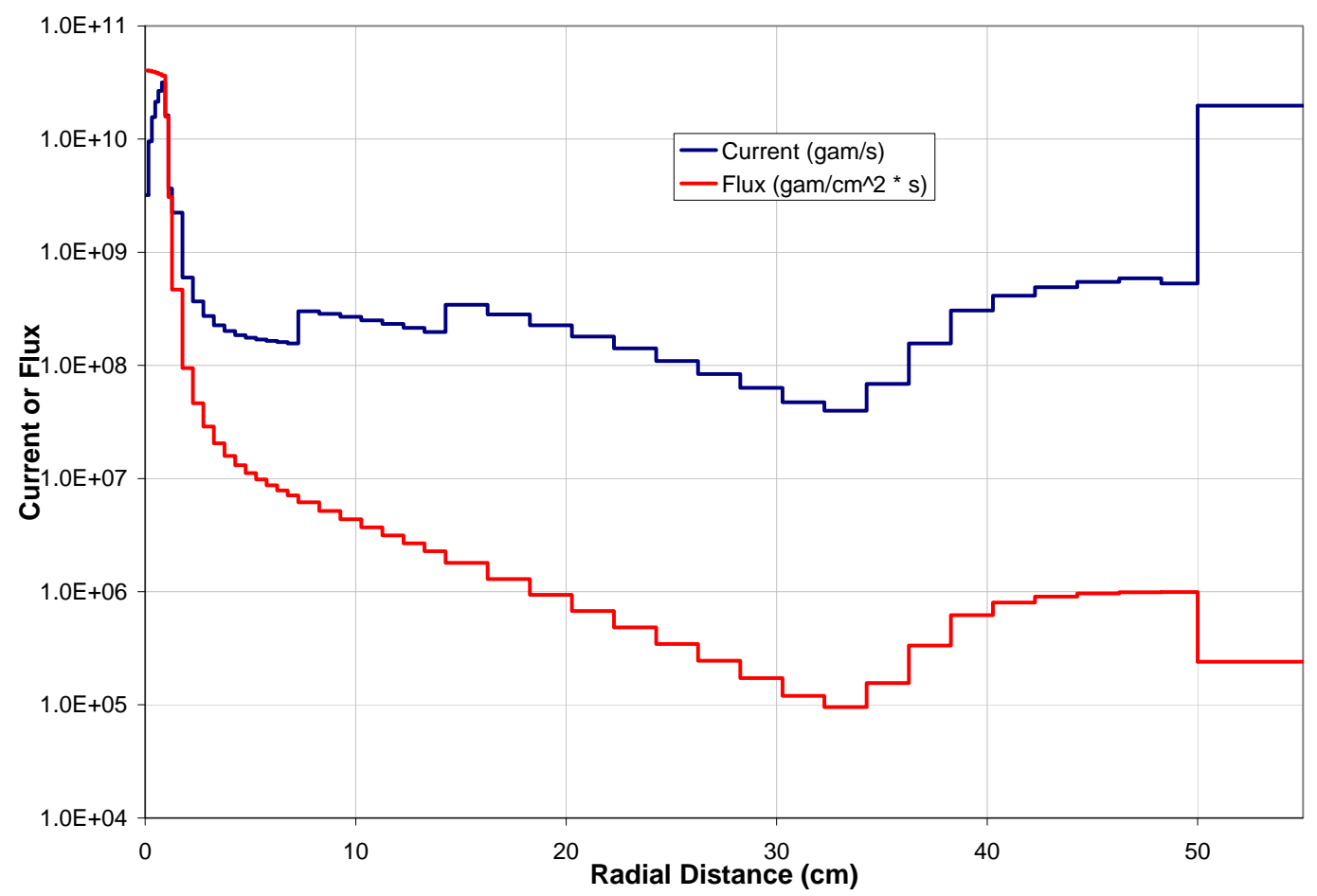

Figure 3. Photon source intensity and flux vs. radial distance from beam axis.

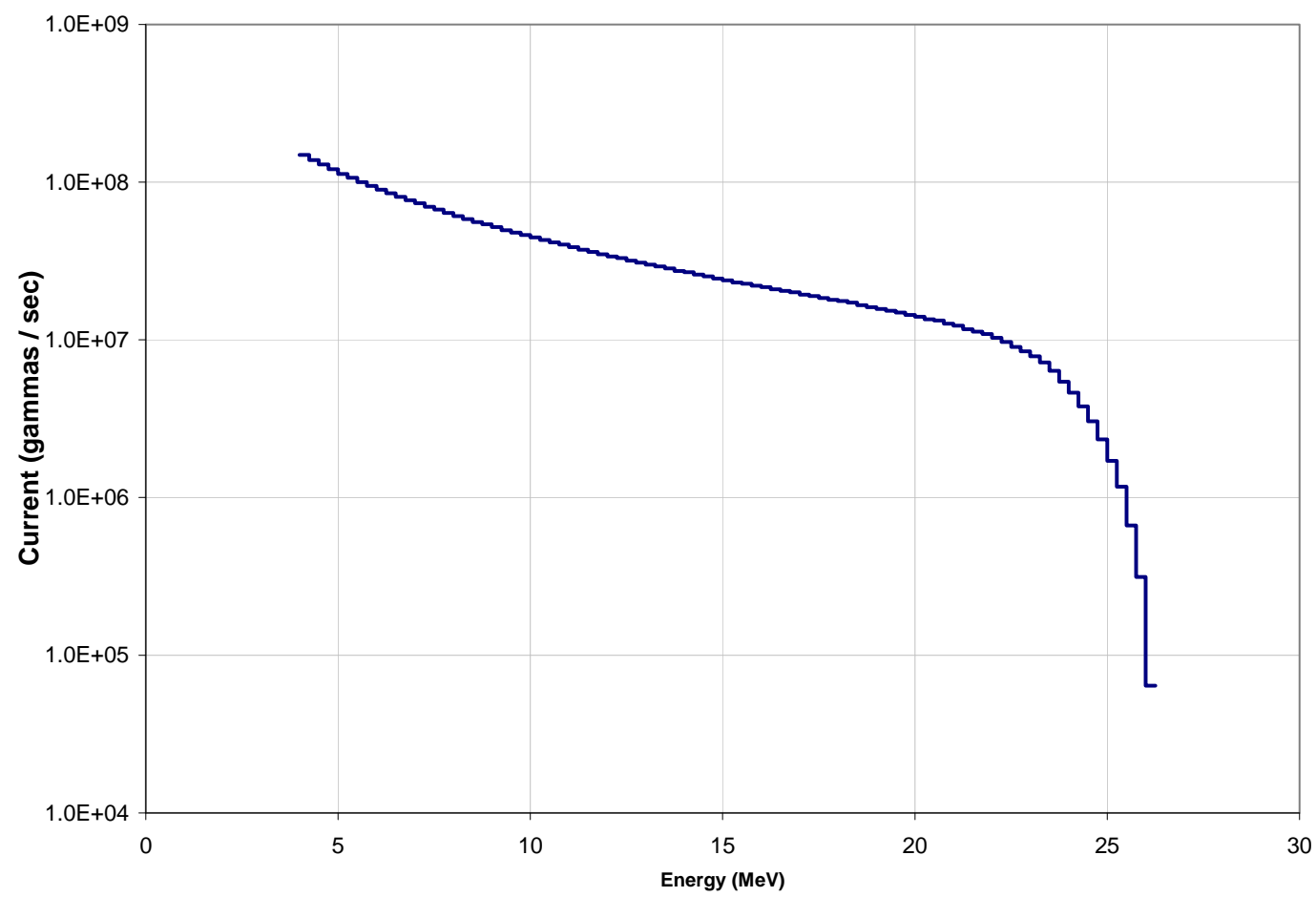

Figure 4. Photon source current for the first radial region $(\mathrm{R}<0.15875 \mathrm{~cm})$ vs. energy. 


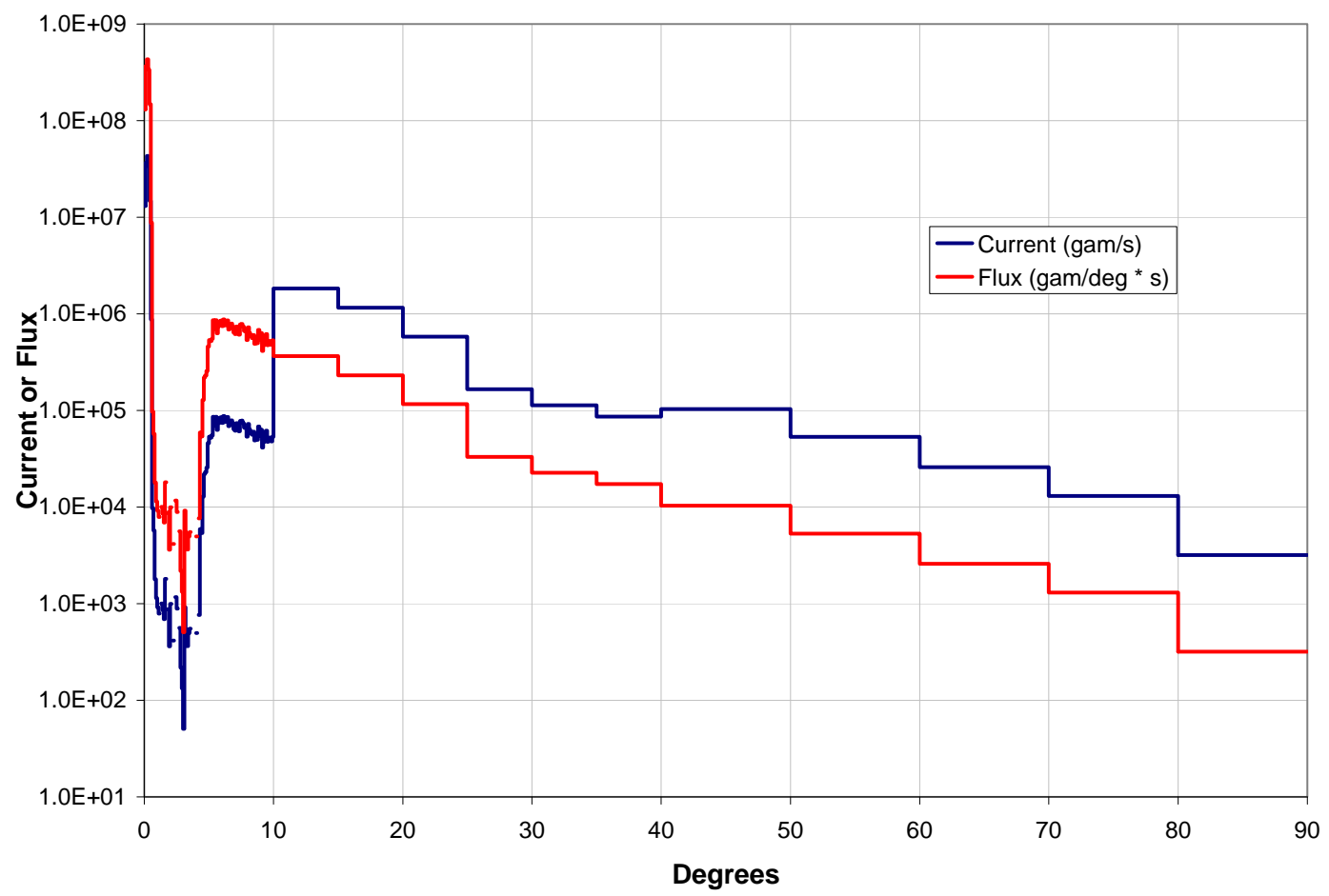

Figure 5. Photon source current and flux for the first radial region $(\mathrm{R}<0.15875 \mathrm{~cm})$ and first energy group (4 MeV $<\mathrm{E}<4.25 \mathrm{MeV}$ ) vs. degrees from the beam axis.

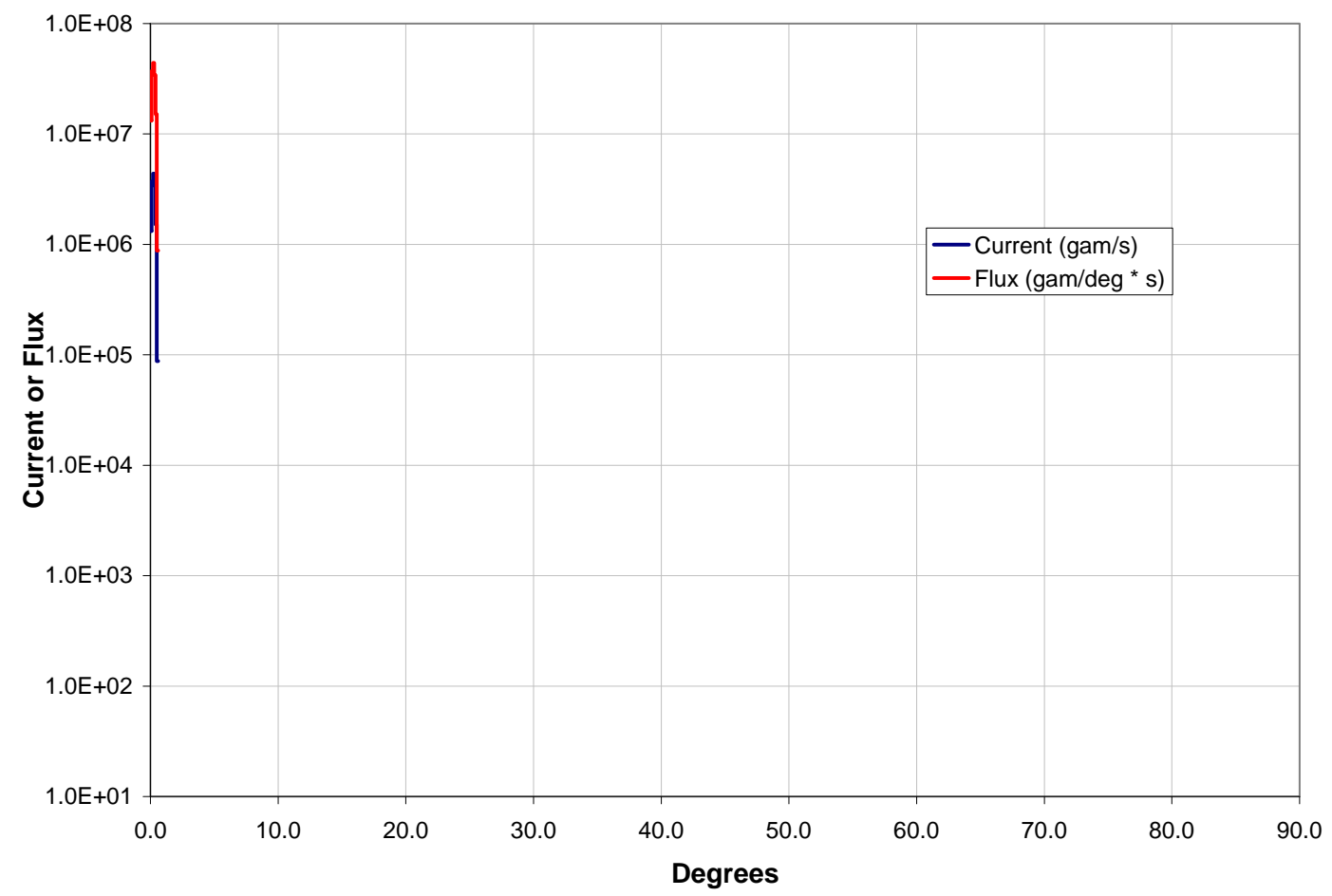

Figure 6. Photon source current and flux for the first radial region $(\mathrm{R}<0.15875 \mathrm{~cm})$ and $64^{\text {th }}$ energy group $(19.75 \mathrm{MeV}<\mathrm{E}<20 \mathrm{MeV})$ vs. degrees from the beam axis. 


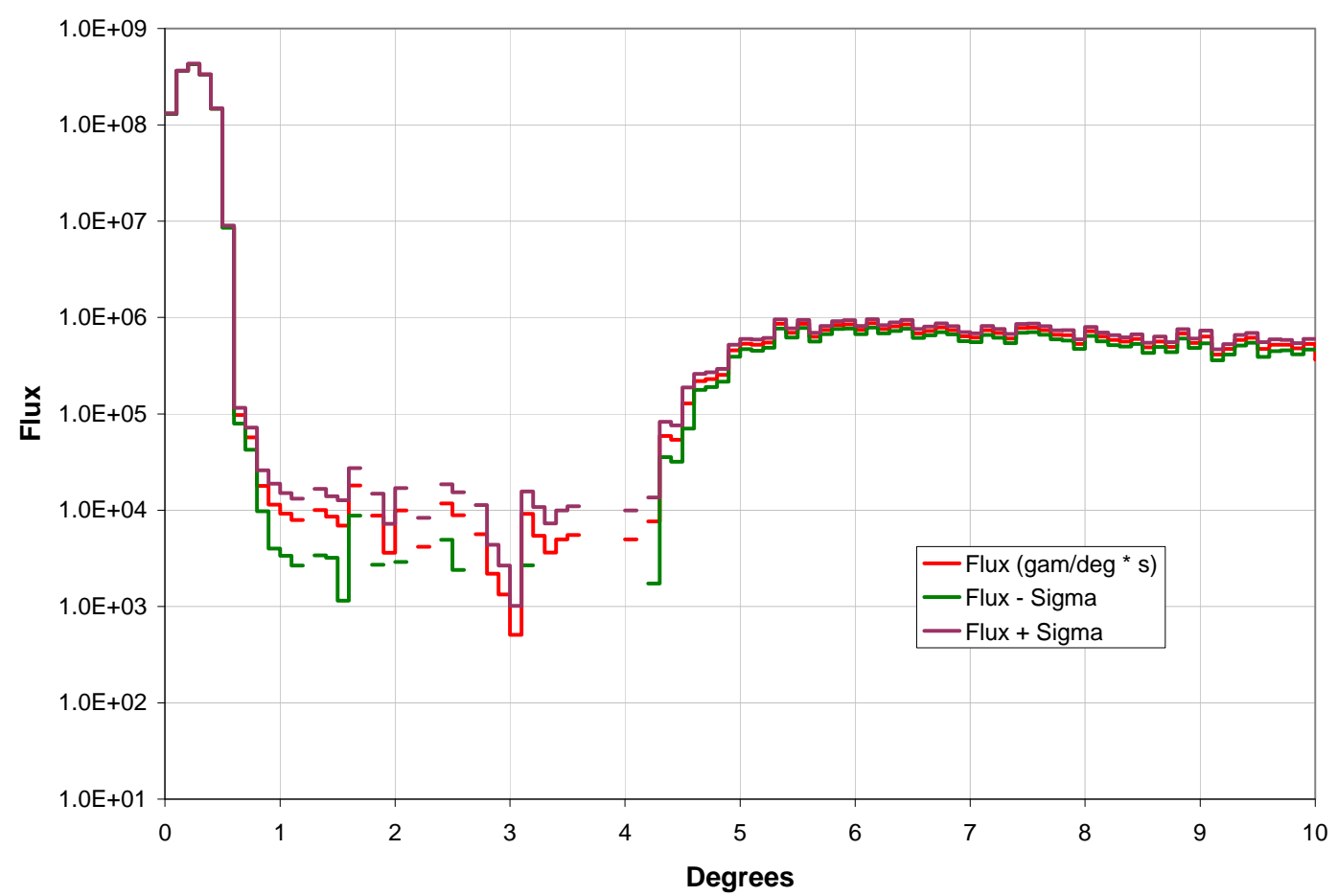

Figure 7. Photon source current and flux for the first radial region $(\mathrm{R}<0.15875 \mathrm{~cm})$ and first energy group ( $4 \mathrm{MeV}<\mathrm{E}<4.25 \mathrm{MeV}$ ) vs. degrees from the beam axis, 0 to $10^{\circ}$.

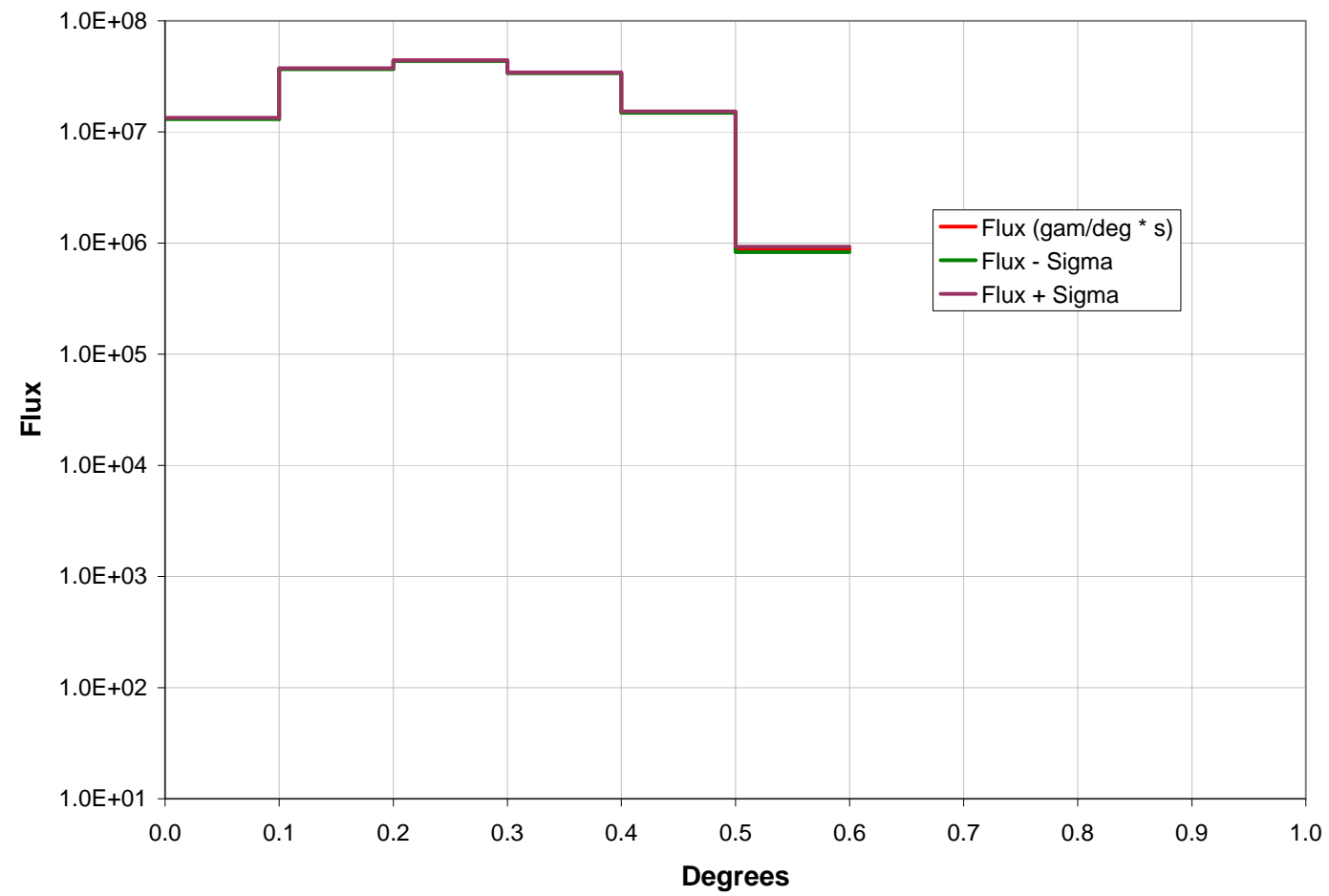

Figure 8. Photon source current and flux for the first radial region $(R<0.15875 \mathrm{~cm})$ and $64^{\text {th }}$ energy group (19.75 $\mathrm{MeV}<\mathrm{E}<20 \mathrm{MeV})$ vs. degrees from the beam axis, 0 to $10^{\circ}$. 


\section{LINAC BEAM CHARACTERIZATION}

\subsection{Angular Spread and Attenuation of Photon Beam}

The photon beam discussed in the previous section was modeled in air both with and without the ground. Then the flux of photons at different distances from the accelerator was calculated to determine how many photons would enter the target at a given location. Using this flux, and the photonuclear cross sections, one can estimate the number of photonuclear interactions that would occur in the target as well as calculate the photoneutron production rate.

\subsection{Photon Beam in Air}

The angular spread and attenuation of the photon beam was modeled in air only (the input file is shown in Appendix B). Tallies in MCNPX are used to compute the photon flux at six planes, from 5 to $30 \mathrm{~m}$ in steps of $5 \mathrm{~m}$, all of which were downstream from the collimator and perpendicular to the beam. This is similar to the geometry shown in Figure 2; however, the system was extended to $30 \mathrm{~m}$, and the vehicle and ground were not modeled. The tally at each plane was segmented into 20 different radial regions (i.e., rings - the first region is simply a disc). The outside radii of the rings in centimeters are 10 to 100 by steps of 10 , and 120 to 300 by steps of 20. The peak of the photon fluxes on these six planes can be seen in Figure 9 through Figure 14. The relative errors for Figure 9 through Figure 14 are all less than 0.05 .
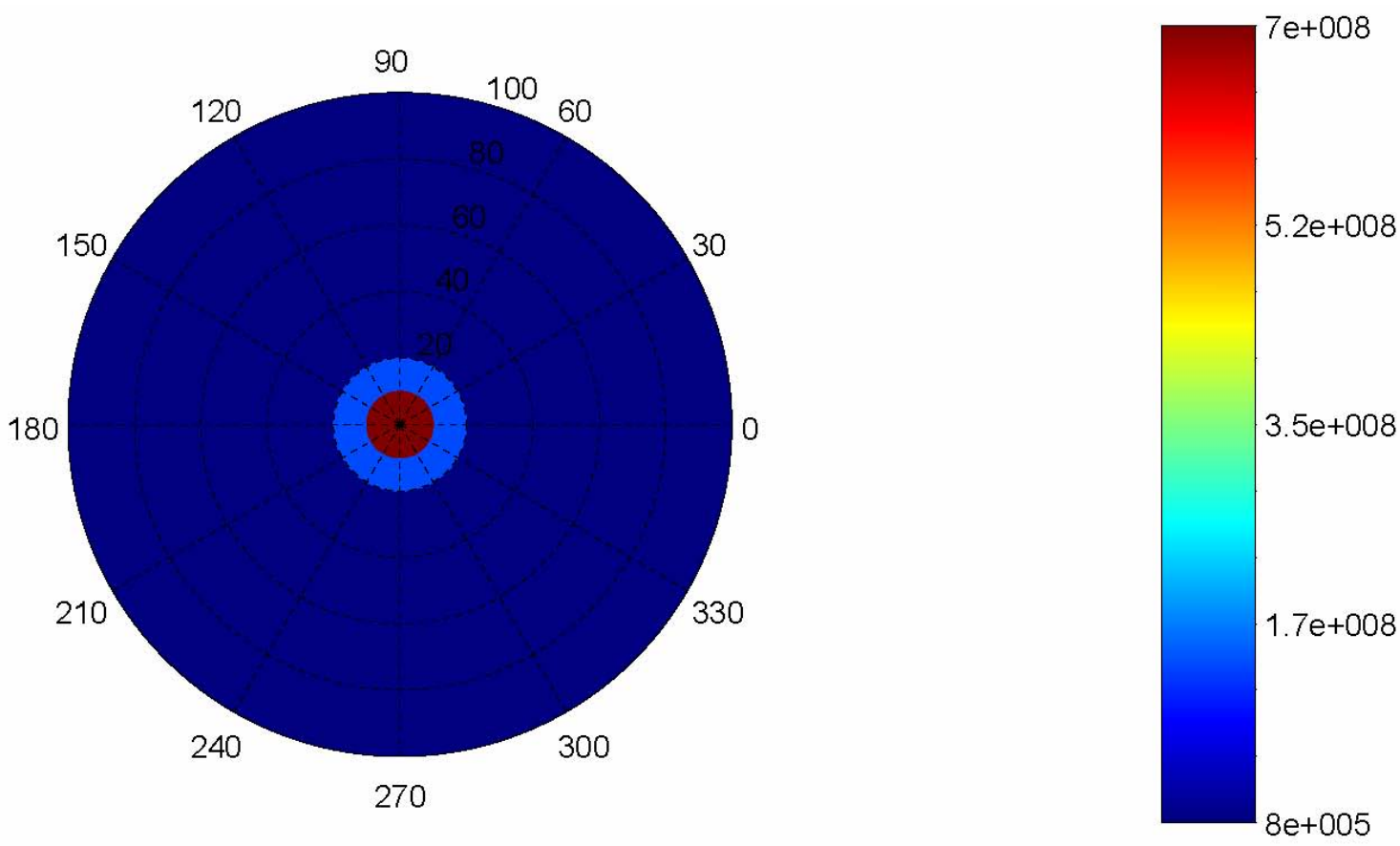

Figure 9. Peak photon flux $\left(\mathrm{cm}^{-2} \mathrm{sec}^{-1}\right) 5 \mathrm{~m}$ from exit of collimator. 

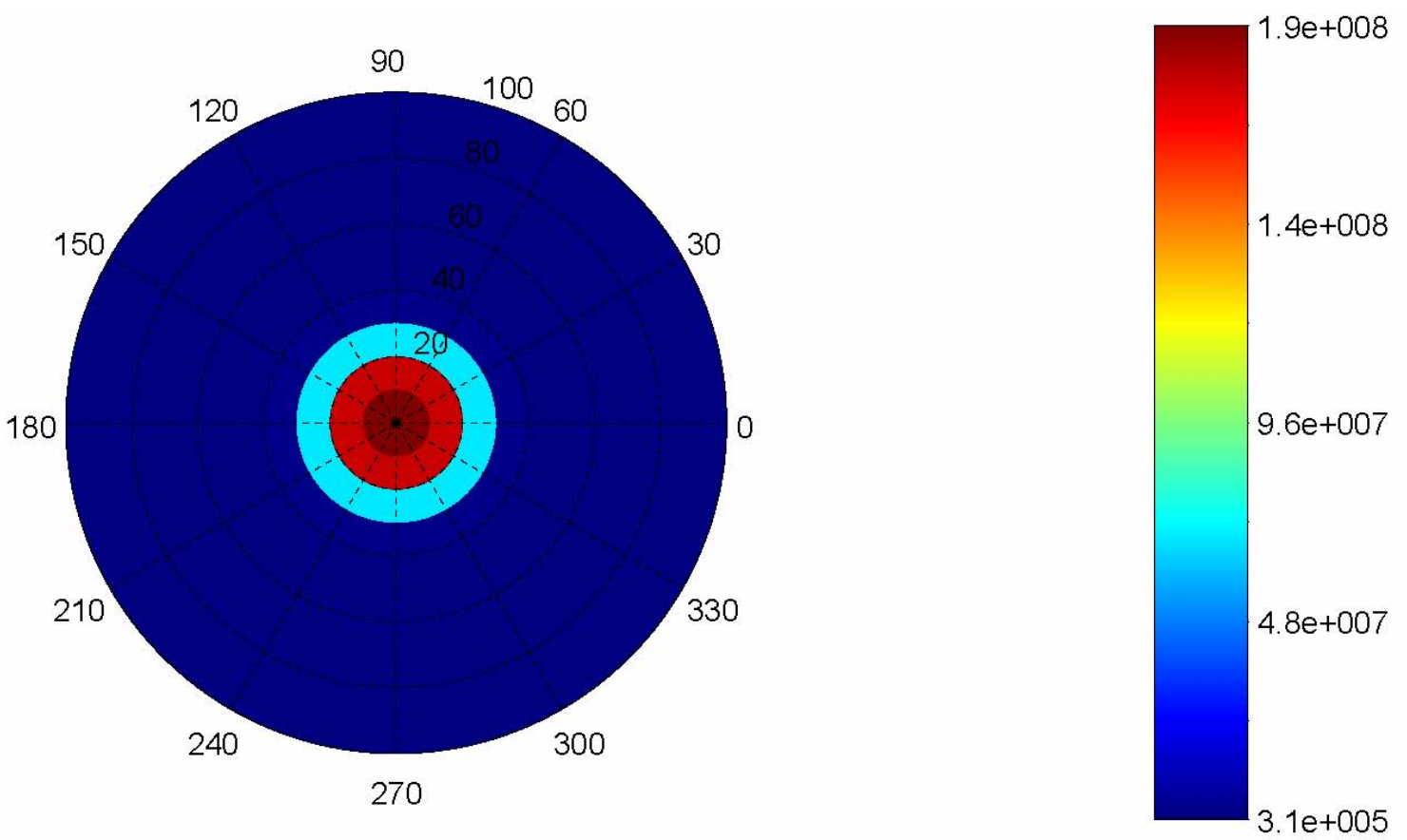

Figure 10. Peak photon flux $\left(\mathrm{cm}^{-2} \mathrm{sec}^{-1}\right) 10 \mathrm{~m}$ from exit of collimator.
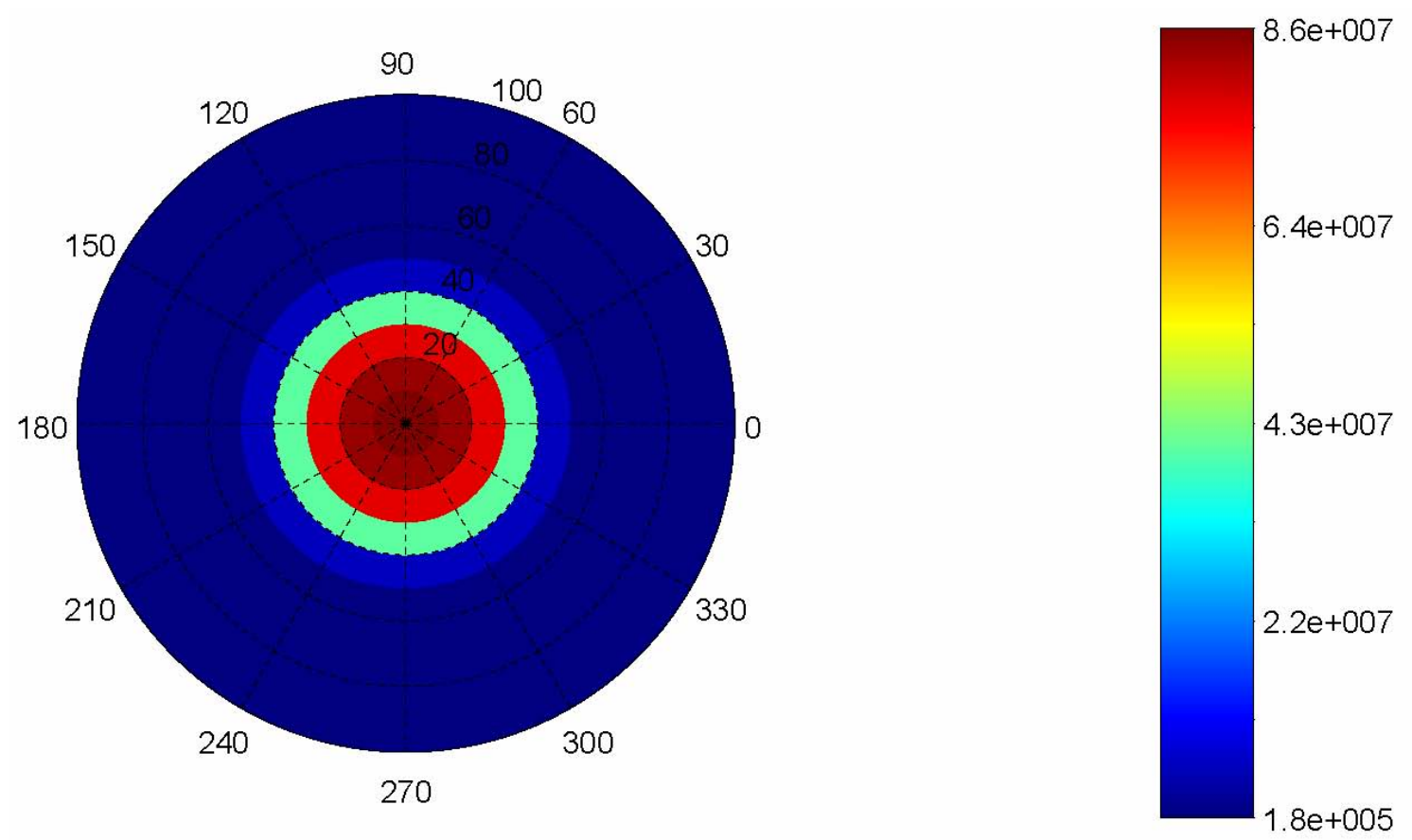

Figure 11. Peak photon flux $\left(\mathrm{cm}^{-2} \mathrm{sec}^{-1}\right) 15 \mathrm{~m}$ from exit of collimator. 

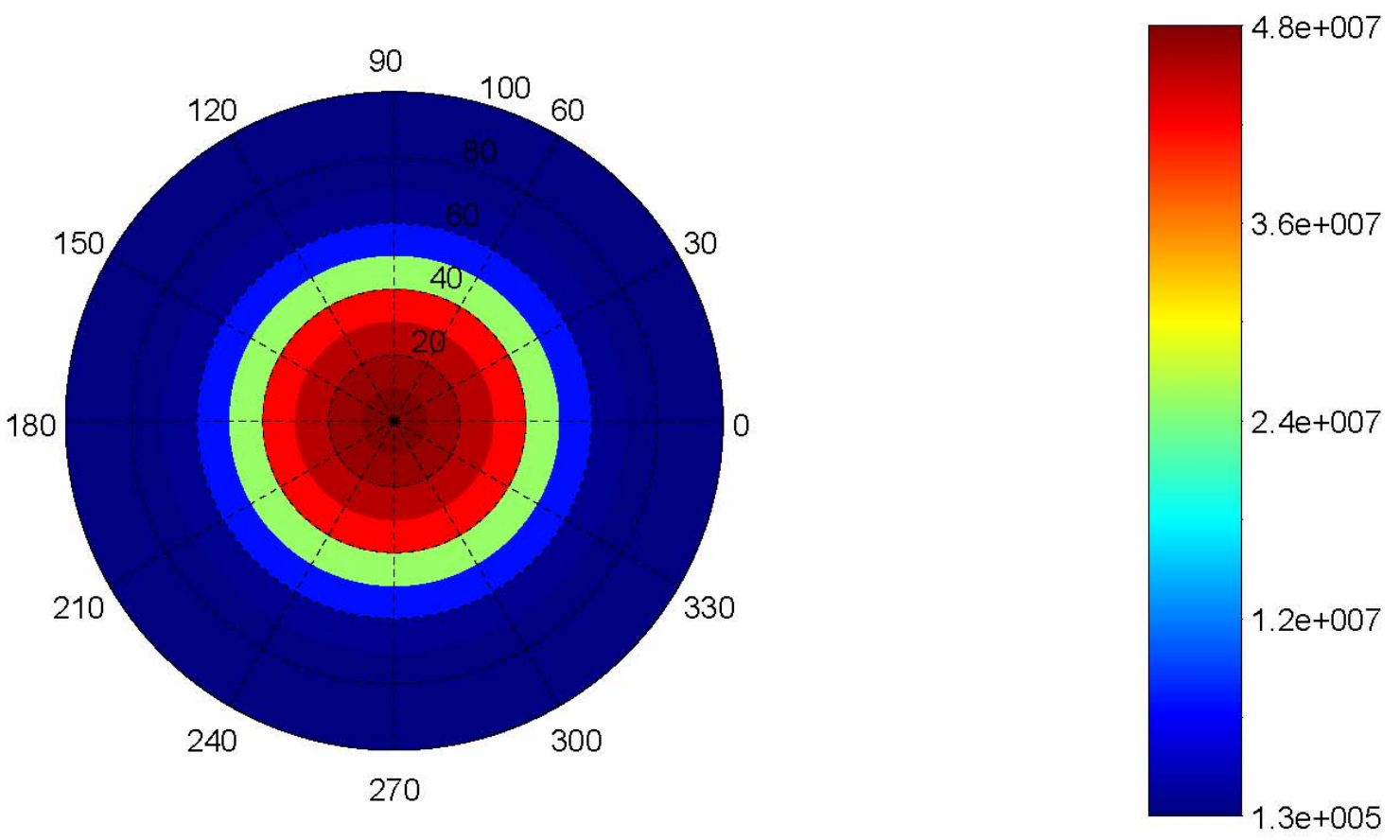

Figure 12. Peak photon flux $\left(\mathrm{cm}^{-2} \mathrm{sec}^{-1}\right) 20 \mathrm{~m}$ from exit of collimator.
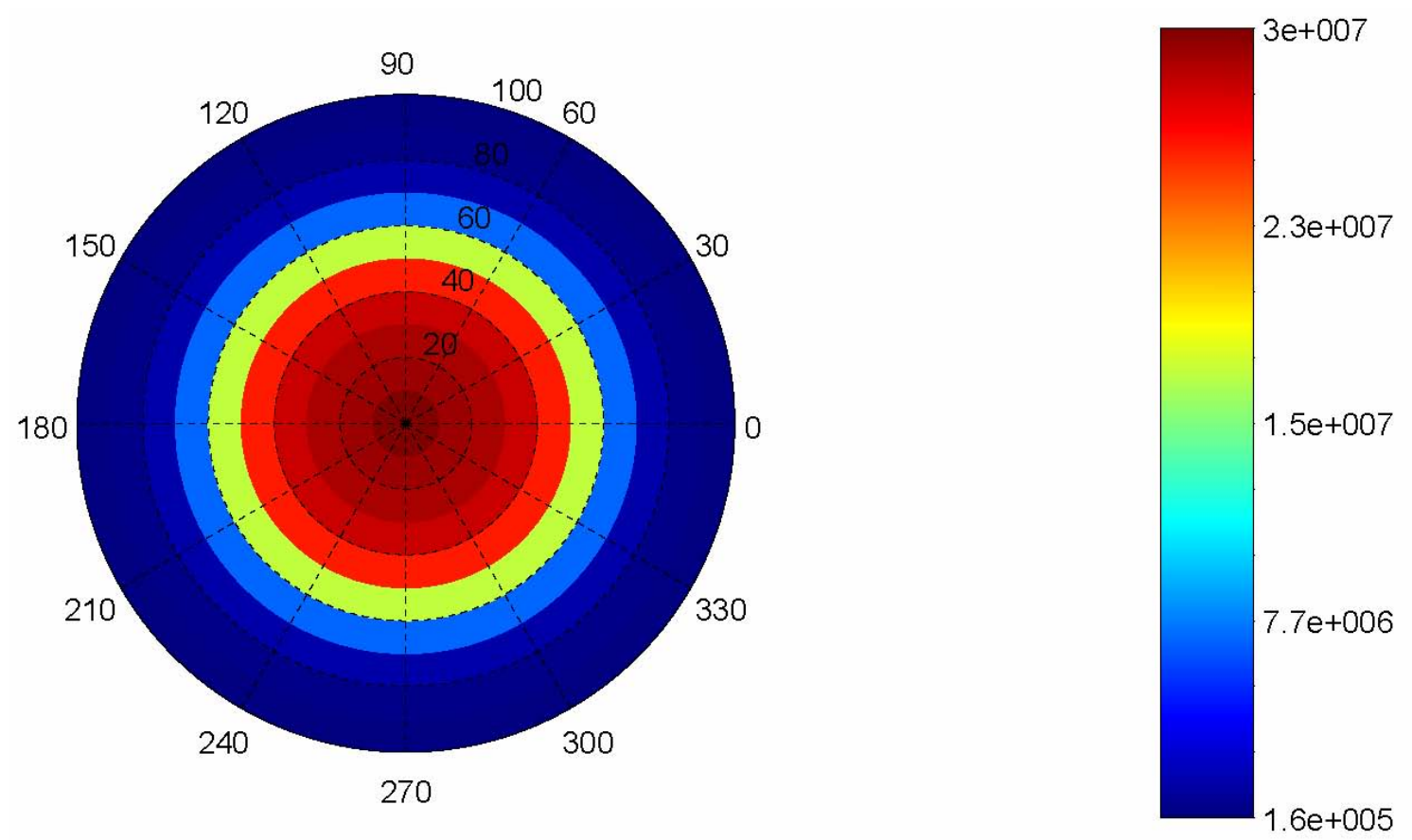

Figure 13. Peak photon flux $\left(\mathrm{cm}^{-2} \mathrm{sec}^{-1}\right) 25 \mathrm{~m}$ from exit of collimator. 

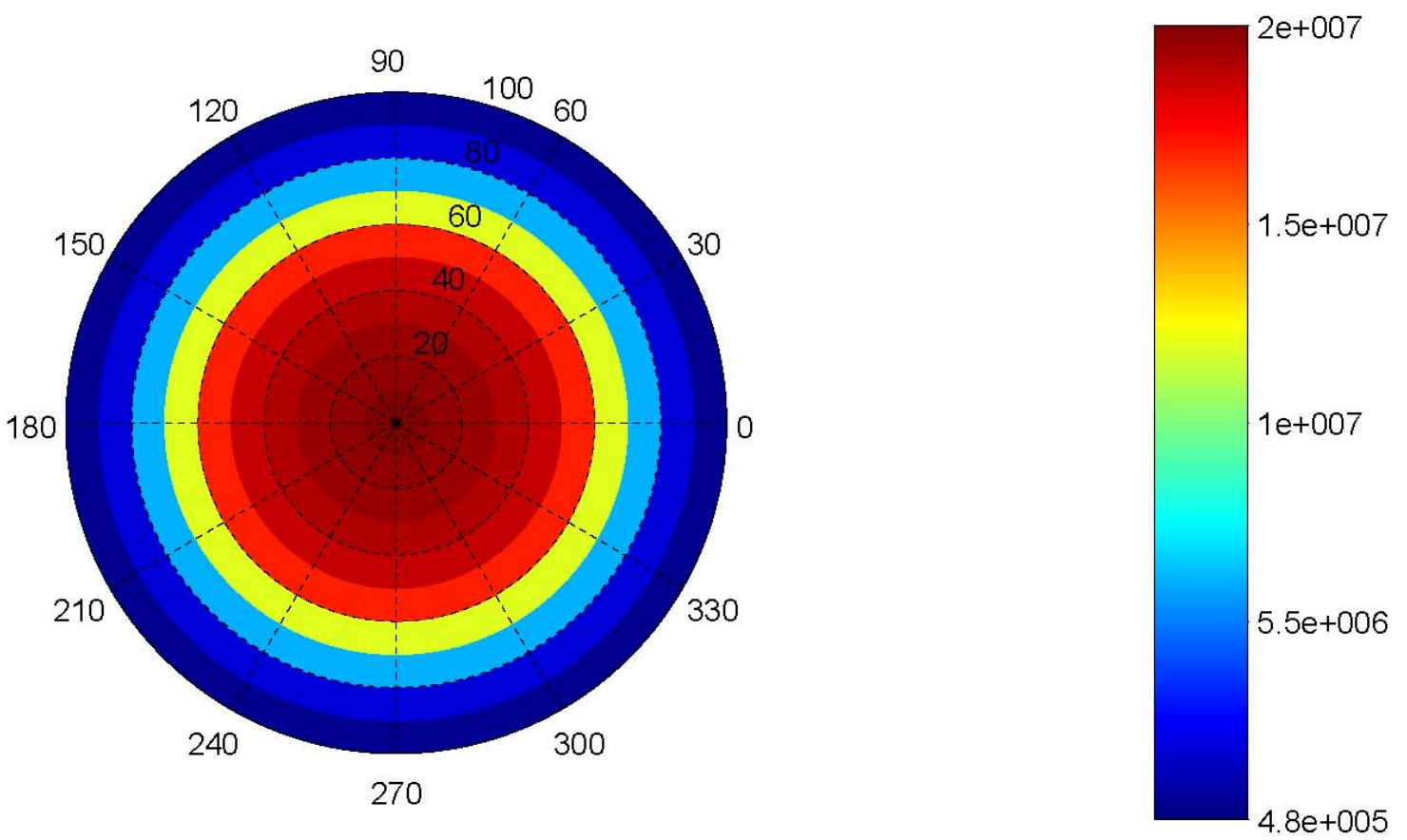

Figure 14. Peak photon flux $\left(\mathrm{cm}^{-2} \mathrm{sec}^{-1}\right) 30 \mathrm{~m}$ from exit of collimator.

The peak photon flux downstream from the collimator shown in Figure 9 through Figure 14 is inside a cone that has a half angle between 1.75 and 2.25 degrees. As a result, a target can be placed at some arbitrary distance from the collimator, and the location of the photon peak with respect to the beam axis is well defined. Table 3 shows the half cone angle of the photon peak at the six tally locations. The exact edge of the photon peak is obscured by the segmenting of the tally flux plane.

Table 3. Half cone angle of peak photon flux

\begin{tabular}{|c|c|c|}
\hline $\begin{array}{c}\text { Distance from } \\
\text { collimator }(\mathrm{cm})\end{array}$ & $\begin{array}{c}\text { Radius of peak } \\
\text { photon flux }(\mathrm{cm})\end{array}$ & $\begin{array}{c}\text { Half cone angle } \\
\text { (degrees) }\end{array}$ \\
\hline 500 & 20 & 2.29 \\
1000 & 30 & 1.72 \\
1500 & 50 & 1.91 \\
2000 & 60 & 1.72 \\
2500 & 80 & 1.83 \\
3000 & 90 & 1.72 \\
\hline
\end{tabular}

\subsection{Photon Beam in Air with Soil}

Next, the angular spread and attenuation of the photon beam was modeled in air and above 1-mthick soil (the input file is shown in Appendix B). The beam axis of the electron accelerator was $1.5 \mathrm{~m}$ above the soil. Unlike the previous calculations, this geometry is not symmetric due to the soil. Therefore, the tallies in MCNPX were segmented into rectangular regions. The photon flux was calculated at the same six planes. The tally at each plane was segmented into 1400 different rectangular regions. Along the $x$-axis (from left to right) the tally had mesh lines from $-300 \mathrm{~cm}$ to 
$-100 \mathrm{~cm}$ by steps of $20 \mathrm{~cm}$; from -100 to 100 by steps of $10 \mathrm{~cm}$; and from 100 to $300 \mathrm{~cm}$ by steps of $20 \mathrm{~cm}$. Along the $y$-axis (bottom to top) the tally had mesh lines from -150 to $100 \mathrm{~cm}$ by steps of $10 \mathrm{~cm}$ and from 100 to $300 \mathrm{~cm}$ by steps of $20 \mathrm{~cm}$. The peak of the photon fluxes on these six planes can be seen below in Figure 15 through Figure 20. The relative errors for Figure 15 through Figure 20 are less than 0.1 in the cells where the photon peak is located and in the cells surrounding the photon peak. Outside the area immediately surrounding the photon peak the relative errors can be as large as 0.8 close to the boundaries of the tally but most are less than 0.2 . The occurrence of these large relative errors explains why the minimum flux labeled in Figures 15 and 16 is approximately $7 \mathrm{\gamma} / \mathrm{cm}^{2} / \mathrm{sec}$. This value is far less than the other minimum flux levels in Figures 12, 13, 14, and 17 and is nonphysical. The minimum flux values in Figures 15 and 16 should be on the order of $1 \mathrm{e} 3$.

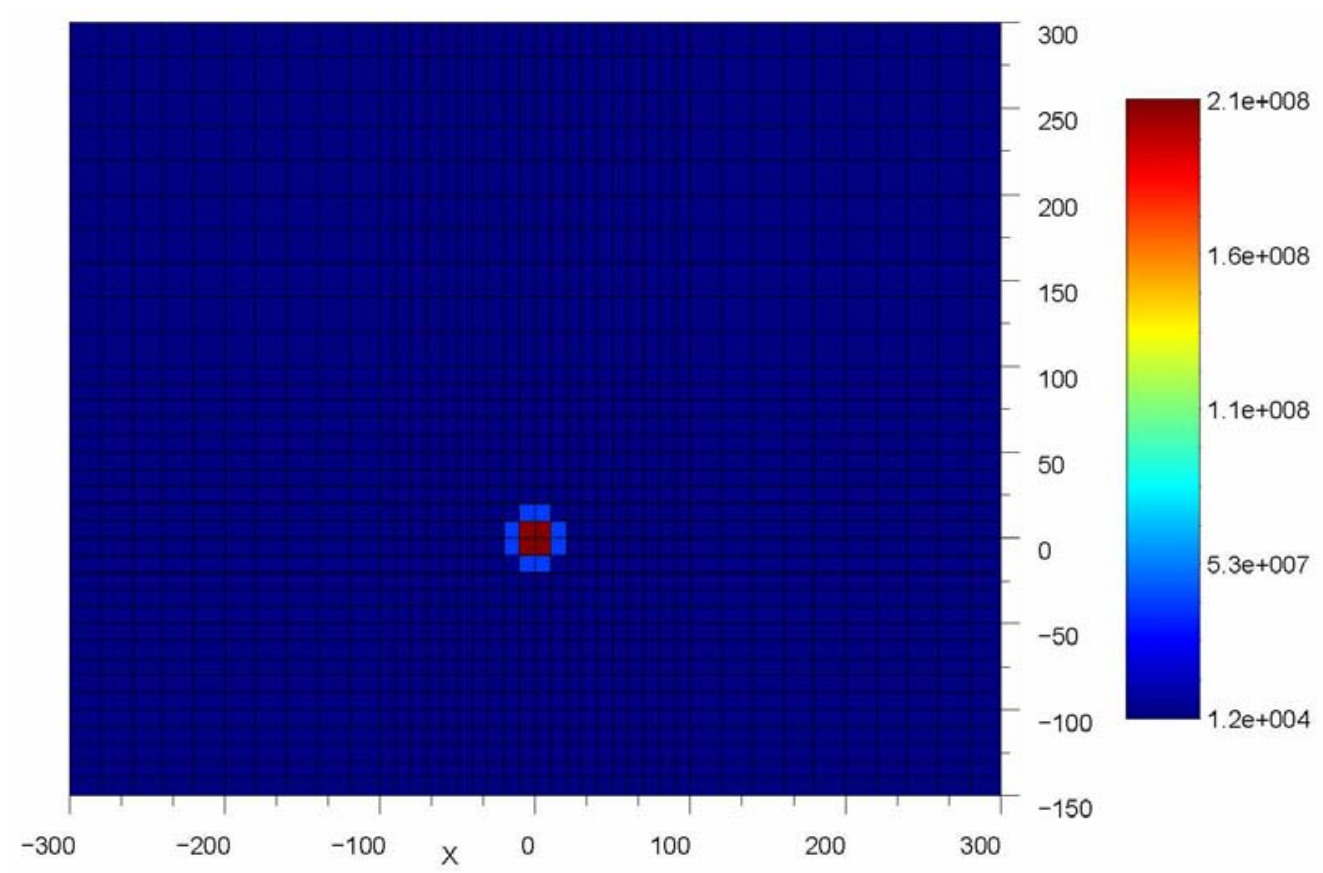

Figure 15. Peak photon flux $\left(\mathrm{cm}^{-2} \mathrm{sec}^{-1}\right)$ above the soil $5 \mathrm{~m}$ from exit of collimator. 


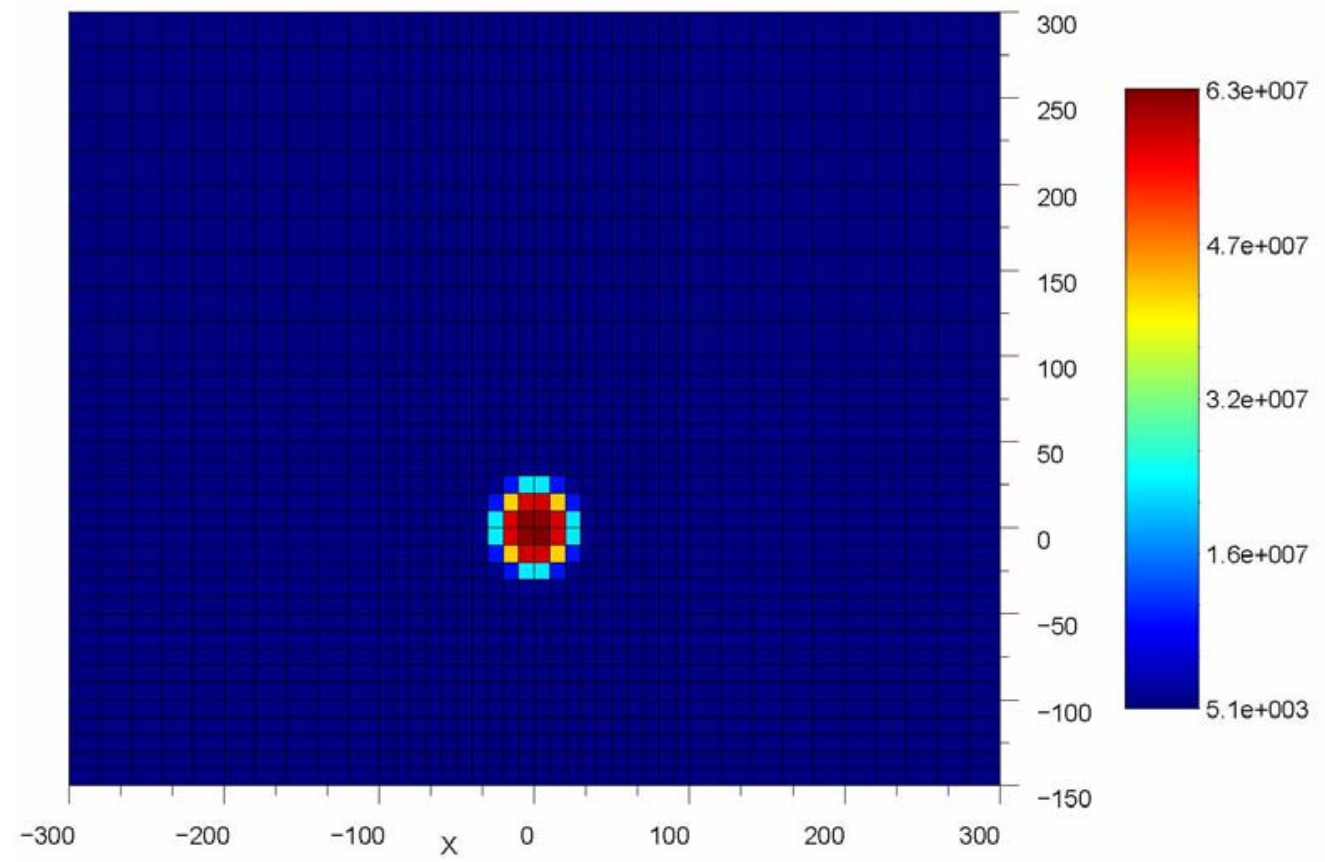

Figure 16. Peak Photon flux $\left(\mathrm{cm}^{-2} \mathrm{sec}^{-1}\right)$ above the soil $10 \mathrm{~m}$ from exit of collimator.

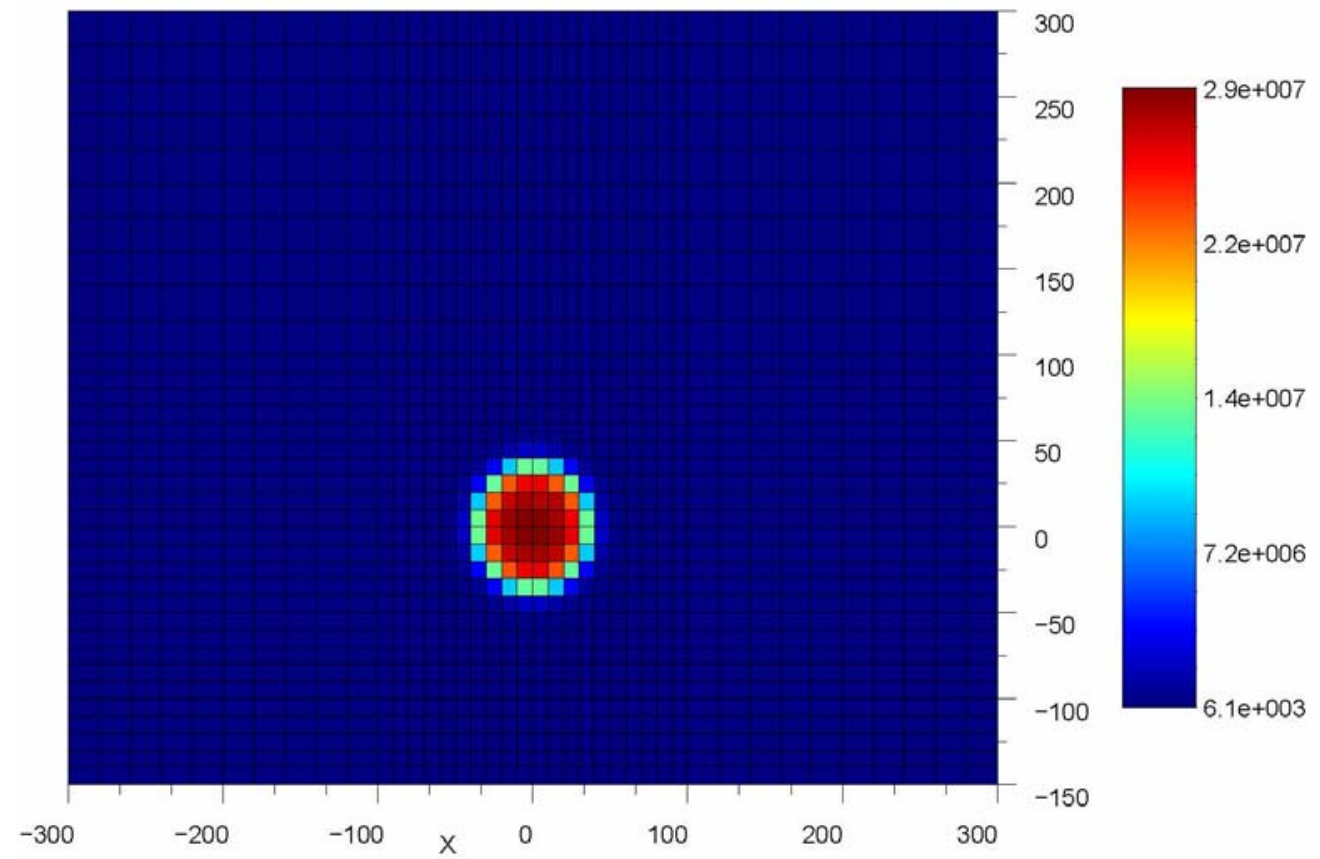

Figure 17. Peak photon flux $\left(\mathrm{cm}^{-2} \mathrm{sec}^{-1}\right)$ above the soil $15 \mathrm{~m}$ from exit of collimator. 


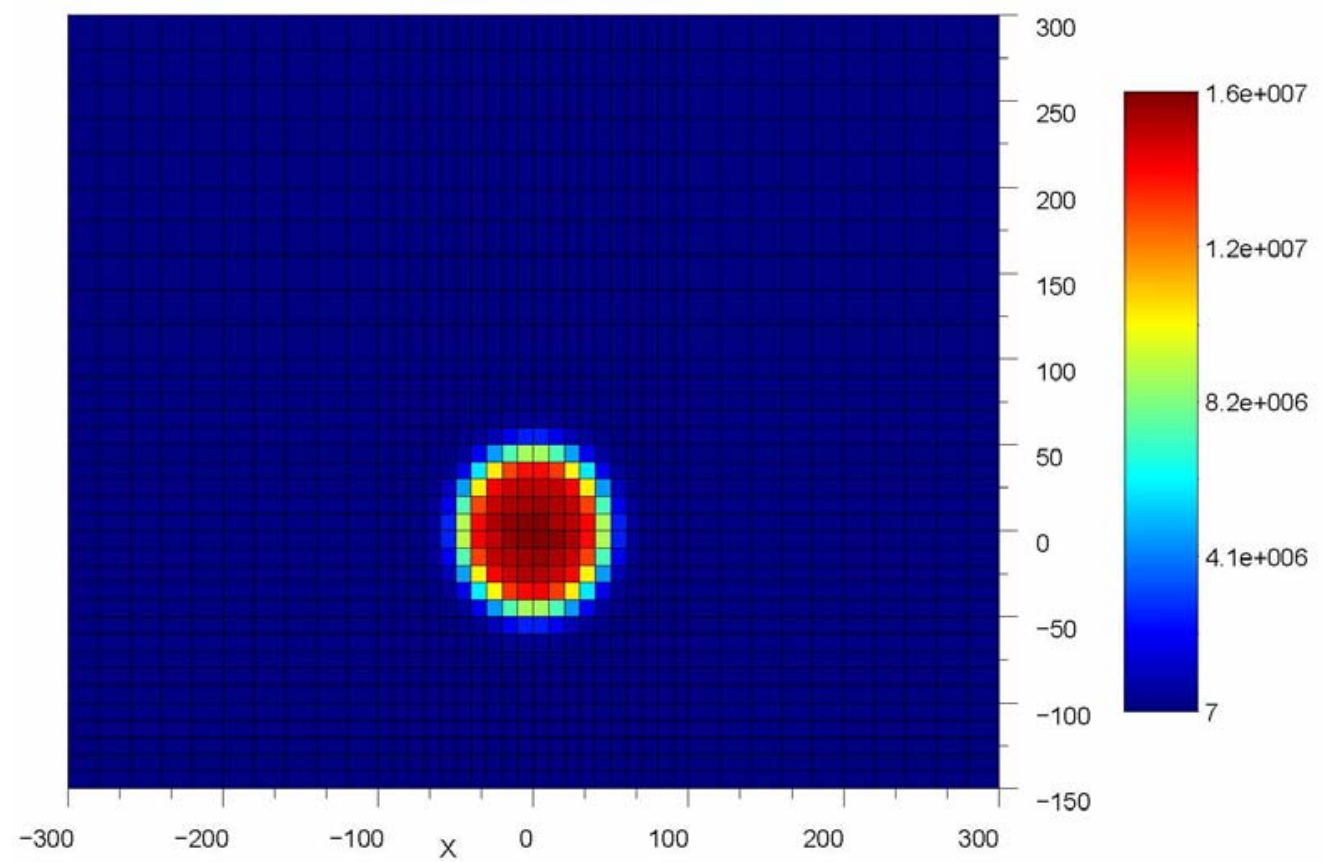

Figure 18. Peak Photon flux $\left(\mathrm{cm}^{-2} \mathrm{sec}^{-1}\right)$ above the soil $20 \mathrm{~m}$ from exit of collimator.

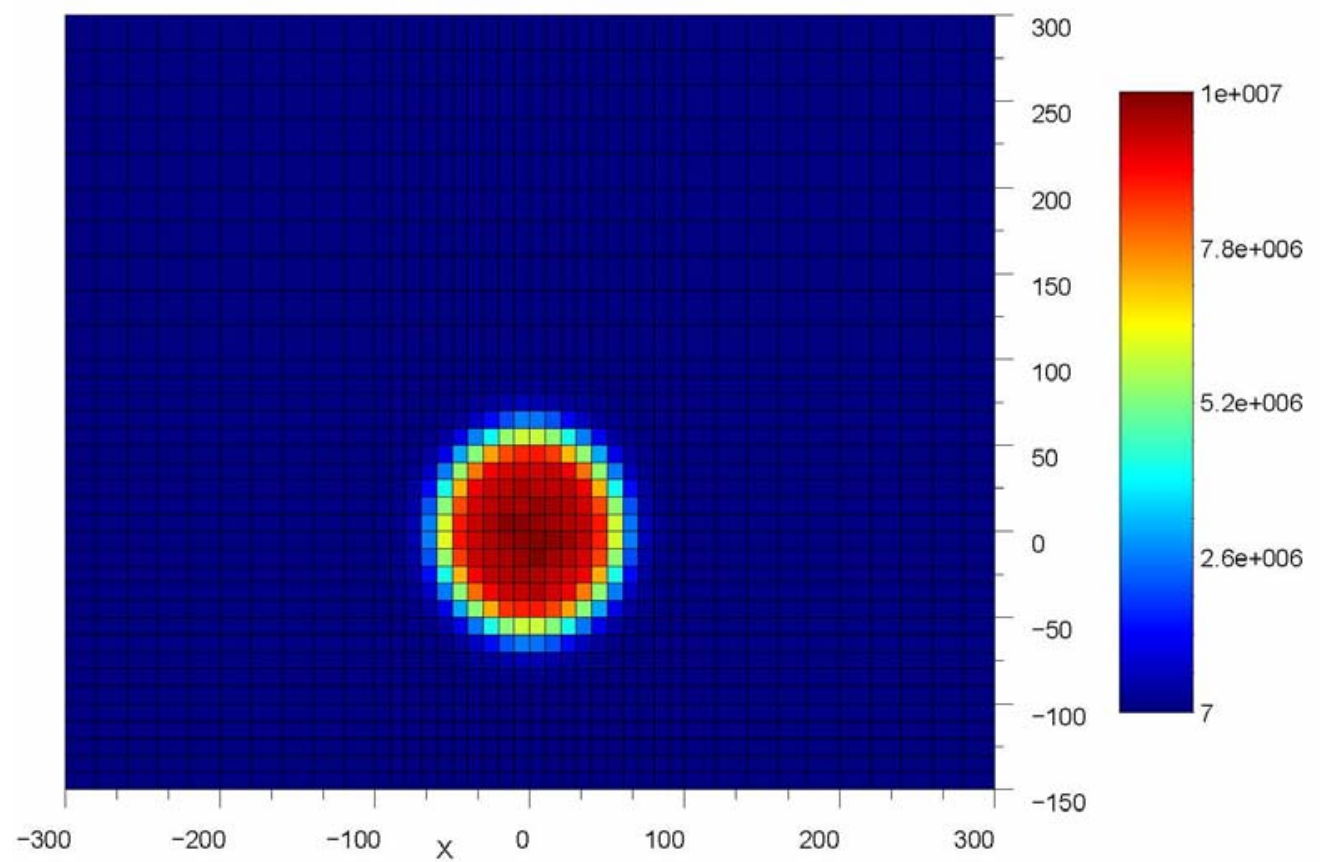

Figure 19. Peak photon flux $\left(\mathrm{cm}^{-2} \mathrm{sec}^{-1}\right)$ above the soil $25 \mathrm{~m}$ from exit of collimator. 


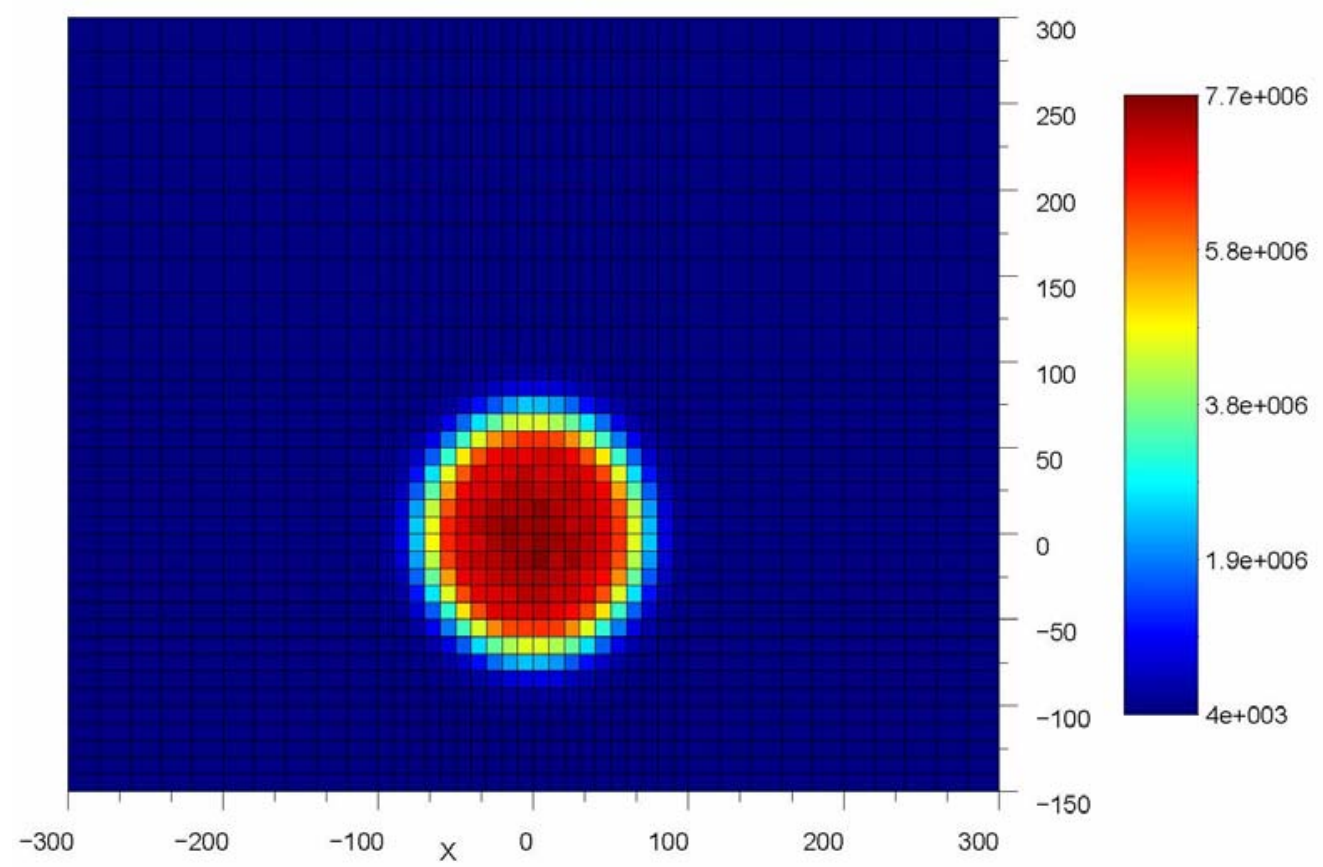

Figure 20. Peak photon flux $\left(\mathrm{cm}^{-2} \mathrm{sec}^{-1}\right)$ above the soil $30 \mathrm{~m}$ from exit of collimator.

In Figure 15 through Figure 20 the peak photon flux downstream from the collimator is still inside a cone that has a half angle between 1.75 and $2.25^{\circ}$. The presence of the ground had practically no effect on the photon flux, at least when compared to the peak flux. This may suggest that it is not necessary to model the ground in these simulations; however, this ultimately is not the case. Indeed, when the neutrons produced in these simulations are considered, the ground will play a more important role.

\section{PROMPT NEUTRON AND PHOTON FLUX MAPS IN MODEL GEOMETRY}

The MCNPX code ver. 2.5 was used for prompt neutron and gamma ray fluence simulations at various horizontal planes in the geometry shown in Figure 2. For the neutron fluence simulations, the mesh cells had a size of $2 \times 2 \times 2 \mathrm{~cm}^{3}$, while for the gamma-ray simulations, the mesh cells were $10 \times 10 \times 10 \mathrm{~cm}^{3}$ in size. The fluence values were averaged over the volume of the mesh cells.

The neutron production relies on photonuclear reactions occurring in the tungsten converter, the collimator, the LINAC shielding, and the depleted uranium target. However, the prompt neutron contribution of the latter is negligible. The primary source of high-energy gamma rays that are required to produce neutrons is bremsstrahlung in the tungsten converter. Bremsstrahlung can also occur in the collimator and shielding; however, many photons produced in these materials will not have sufficient energy to produce photoneutrons.

In order to expedite the simulations, appropriate particle energy cut-offs were applied, identical for both electrons and gamma rays. Specifically, for the neutron fluence simulation the cut-off was set to $4 \mathrm{MeV}$, while for the gamma ray fluence simulations, it was $0.1 \mathrm{MeV}$. 
Figure 21 through Figure 27 show the neutron fluences over horizontal planes at different elevations. The lead collimator, together with the LINAC shielding and the tungsten converter, produce the highest number of prompt neutrons (see Figure 21). This is expected considering the photonuclear cross sections for lead and tungsten. As expected, the neutron fluence decreases with decreasing plane elevation. The minimum values are reached at an elevation of $-149 \mathrm{~cm}$ (see Figure 27); this plane is located next to the ground. Appendix D shows the relative errors corresponding to the neutron fluence maps in Figure 21 through Figure 27. The errors reach relatively high values $(\sim 30 \%)$ only on the periphery of the geometry where statistical accuracy is not important. The values in the central part of the geometry are extremely low.

The flux maps generated with the Monte Carlo model indicate that the detectors should be placed approximately $50 \mathrm{~cm}$ behind the exit of the accelerator (see Figure 22). This position minimizes the number of neutrons coming from the accelerator structure as these neutrons must undergo some scattering collisions first.

Figure 28 through Figure 31 show the gamma ray fluences computed in a similar manner to neutron fluences discussed earlier. However, the mesh cell size was increased to $10 \times 10 \times 10$ $\mathrm{cm}^{3}$. The converter and collimator are the main sources of gamma rays. It is clear from the results that the LINAC shielding made of $3 \mathrm{in}$. of lead is very efficient in eliminating of gamma rays in the directions perpendicular to the primary electron beamline. On the other hand, the collimator efficiently focuses the gamma ray beam towards the target. Appendix D shows the relative errors corresponding to the neutron fluences shown in Figure 28 through Figure 31. As expected, the largest errors $(\sim 80 \%)$ are reached in the corners of the geometry at negative $z$-values where accuracy is of low importance. On the other hand, the error values in the central part of the geometry are extremely low.

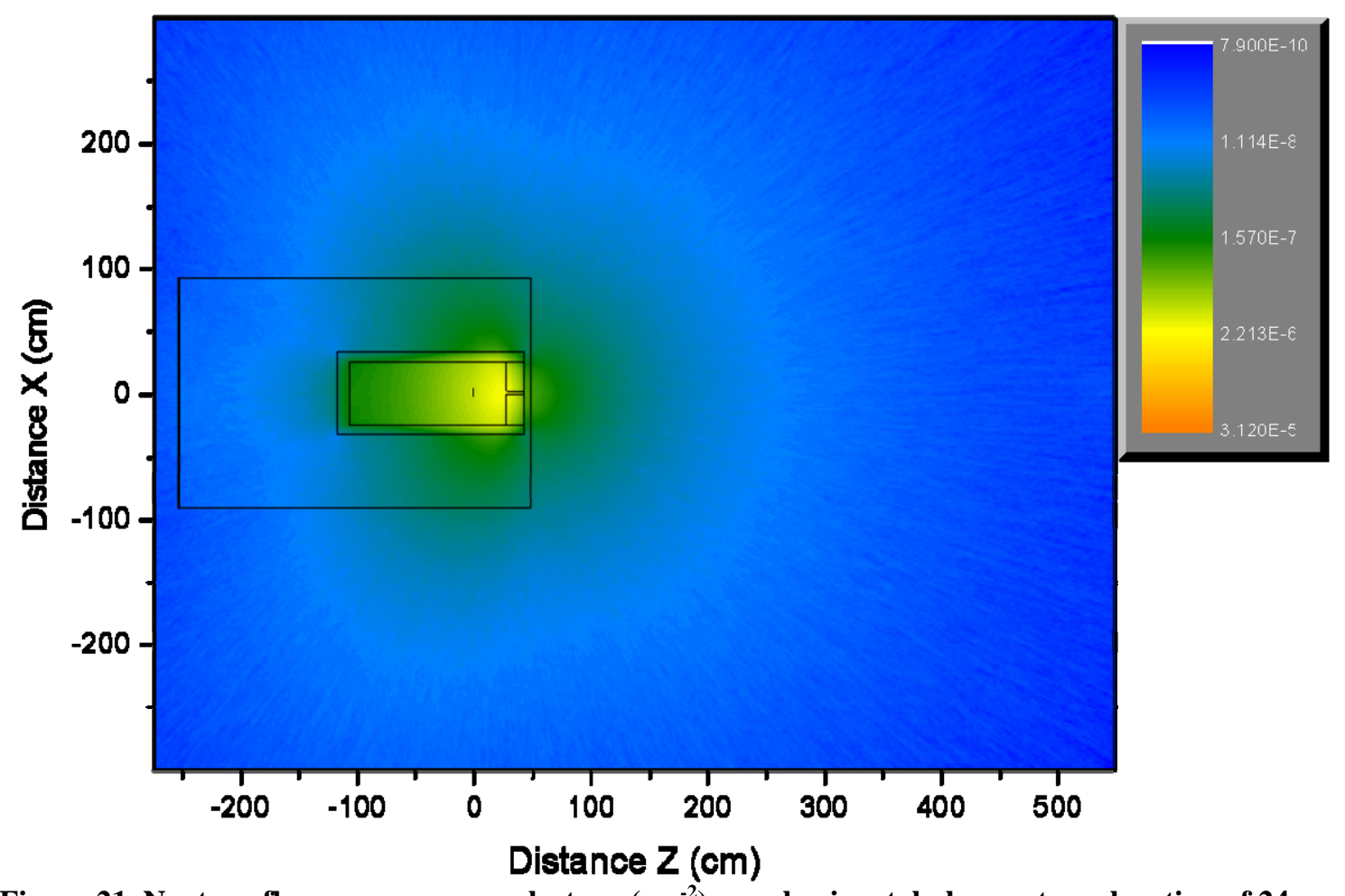

Figure 21. Neutron fluence per source electron $\left(\mathrm{cm}^{-2}\right)$ over horizontal planes at an elevation of $24 \mathrm{~cm}$. 


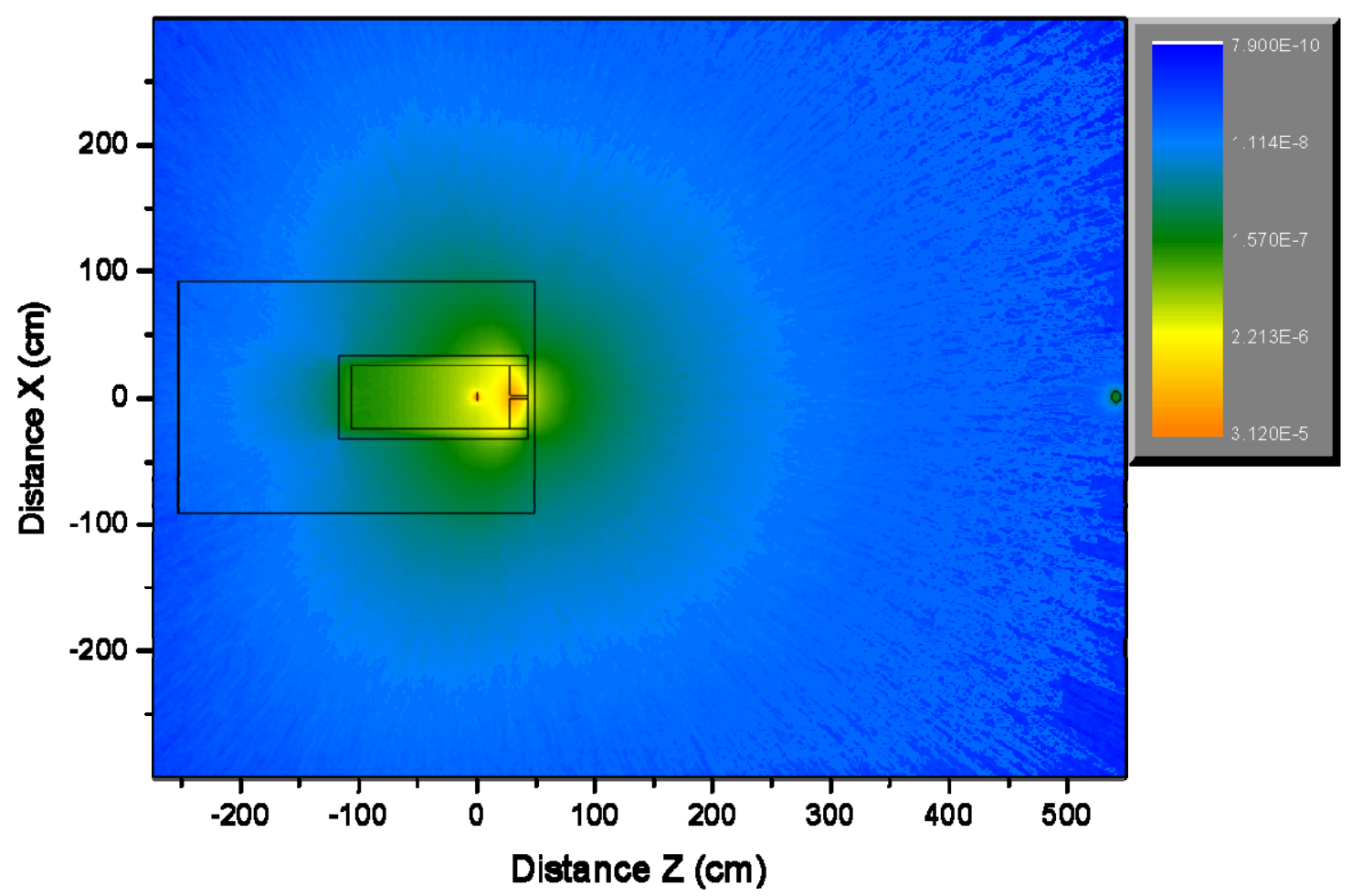

Figure 22. Neutron fluence per source electron $\left(\mathrm{cm}^{-2}\right)$ over horizontal planes at an elevation of $0 \mathrm{~cm}$.

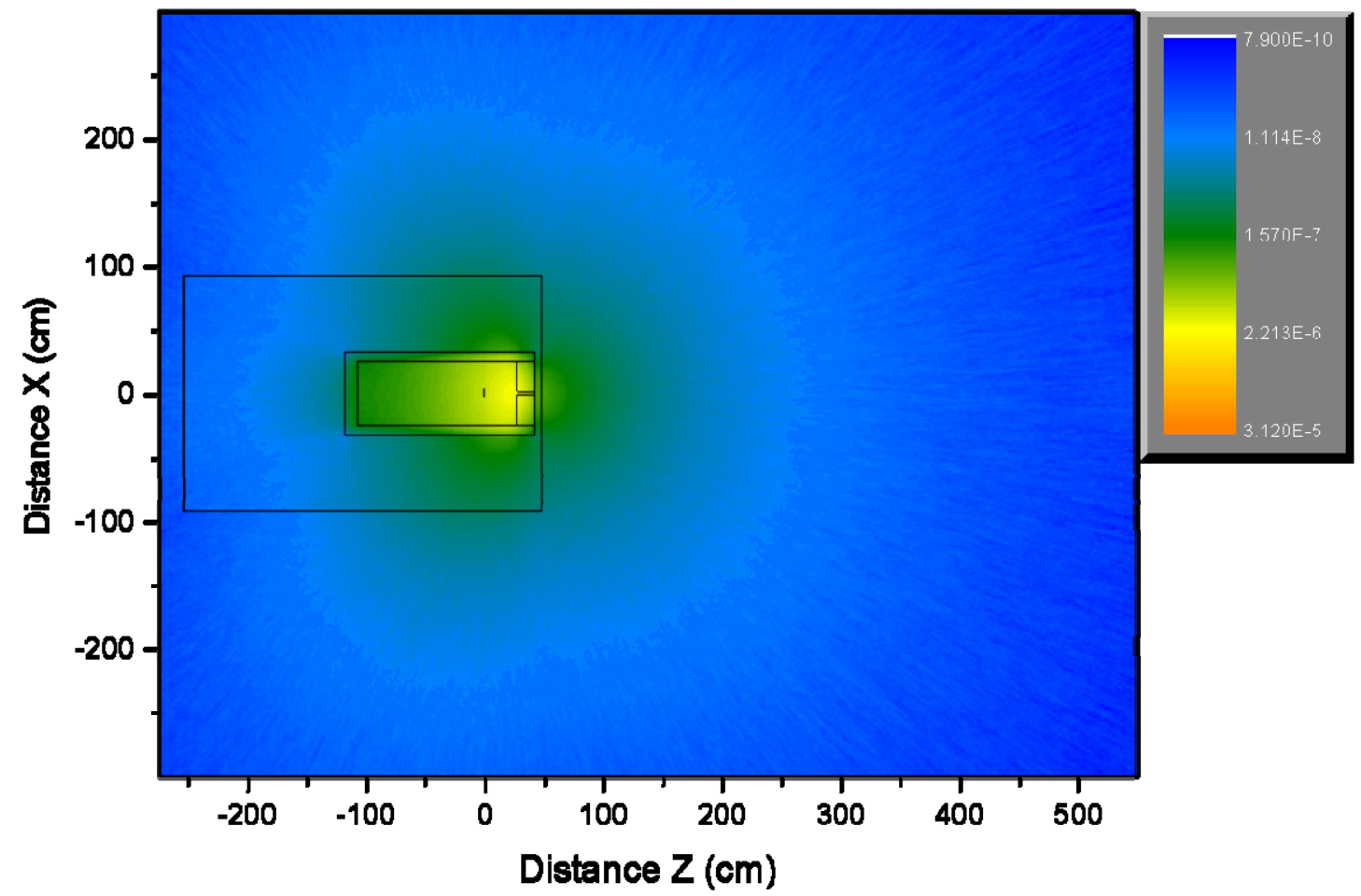

Figure 23. Neutron fluence per source electron $\left(\mathrm{cm}^{-2}\right)$ over horizontal planes at an elevation of $-24 \mathrm{~cm}$. 


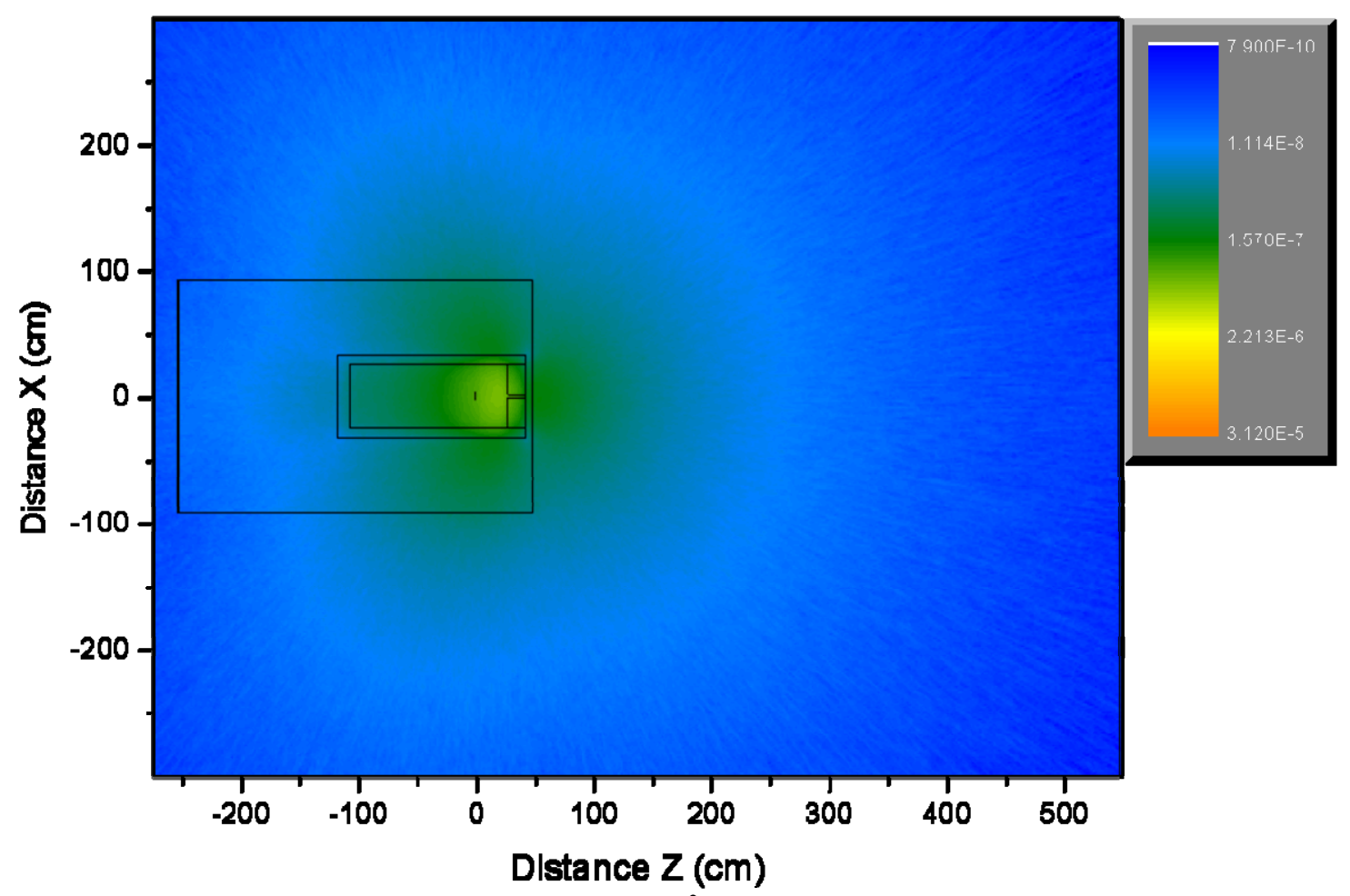

Figure 24. Neutron fluence per source electron $\left(\mathrm{cm}^{-2}\right)$ over horizontal planes at an elevation of $-33.62 \mathrm{~cm}$.

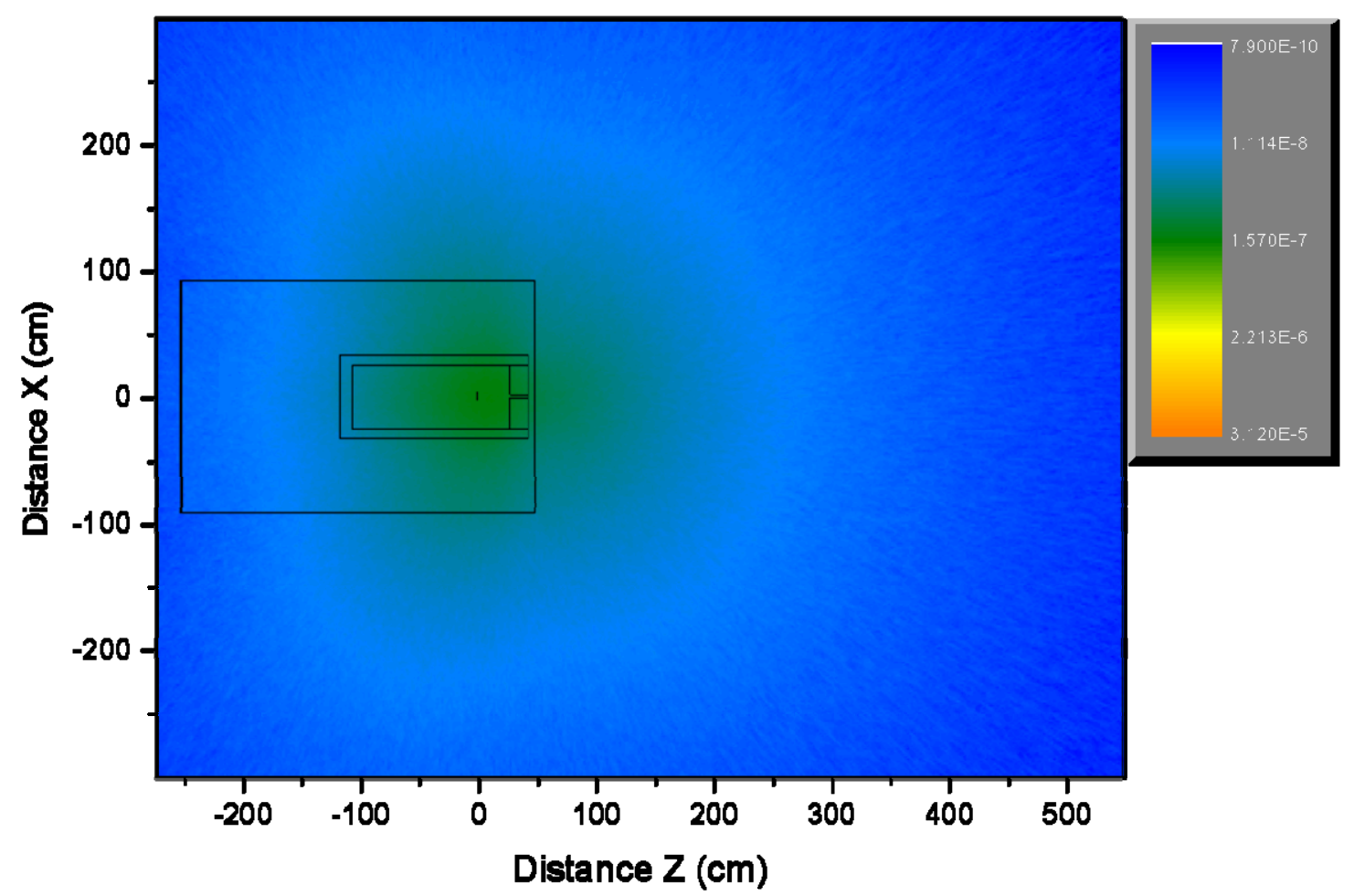

Figure 25. Neutron fluence per source electron $\left(\mathrm{cm}^{-2}\right)$ over horizontal planes at an elevation of $-62 \mathrm{~cm}$. 


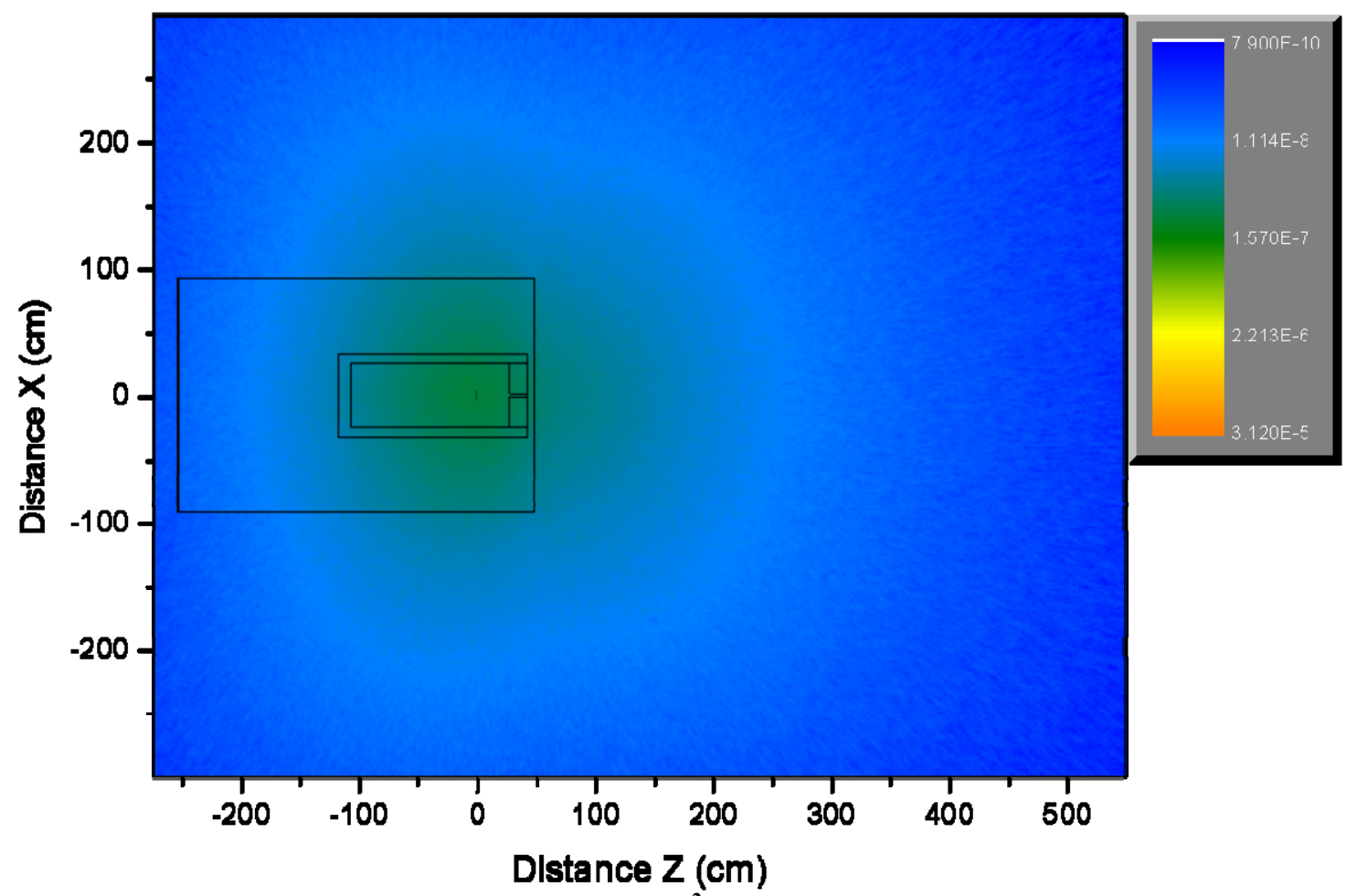

Figure 26. Neutron fluence per source electron $\left(\mathrm{cm}^{-2}\right)$ over horizontal planes at an elevation of $-90.44 \mathrm{~cm}$.

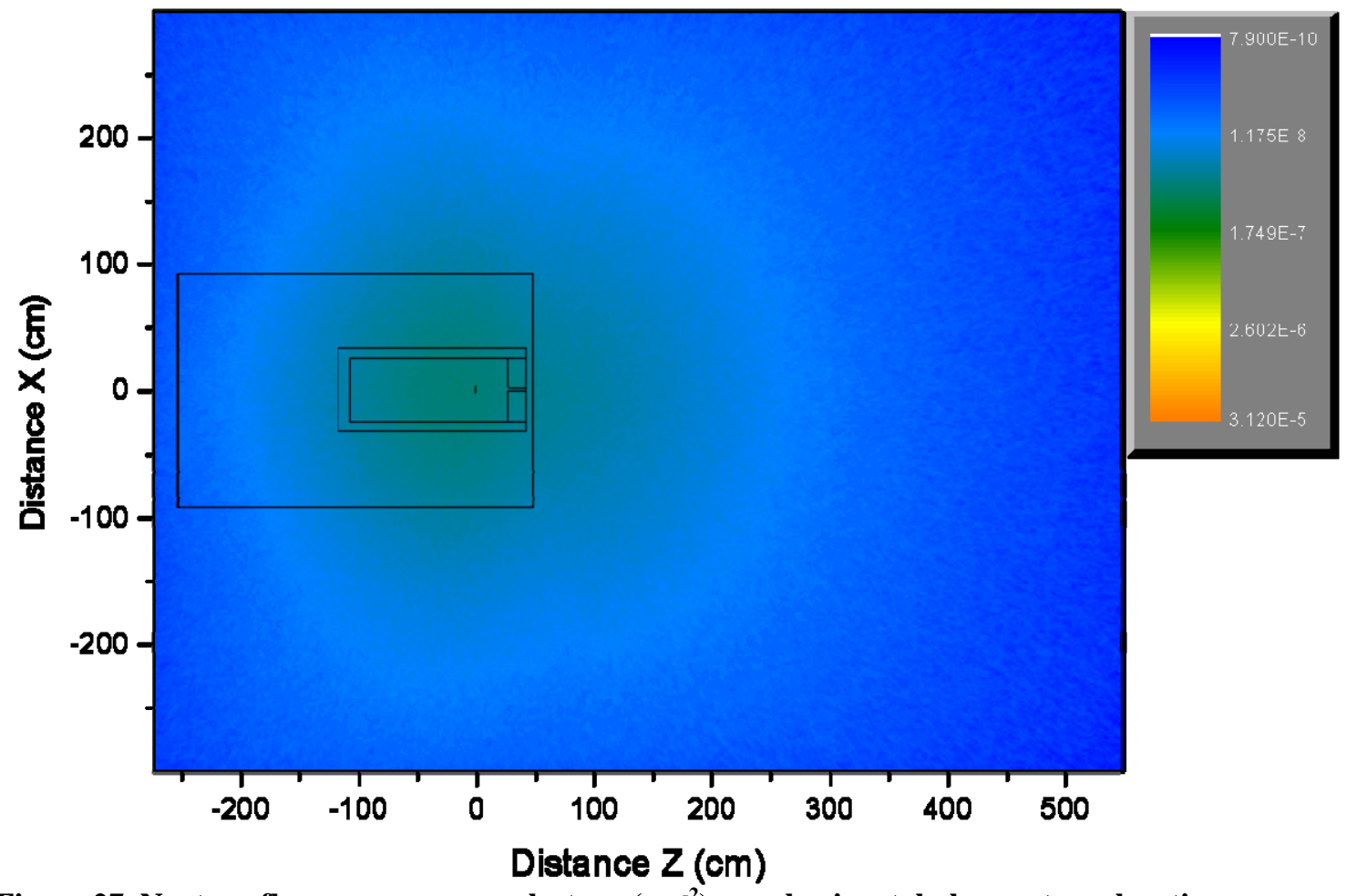

Figure 27. Neutron fluence per source electron $\left(\mathrm{cm}^{-2}\right)$ over horizontal planes at an elevation of $-149 \mathrm{~cm}$. 


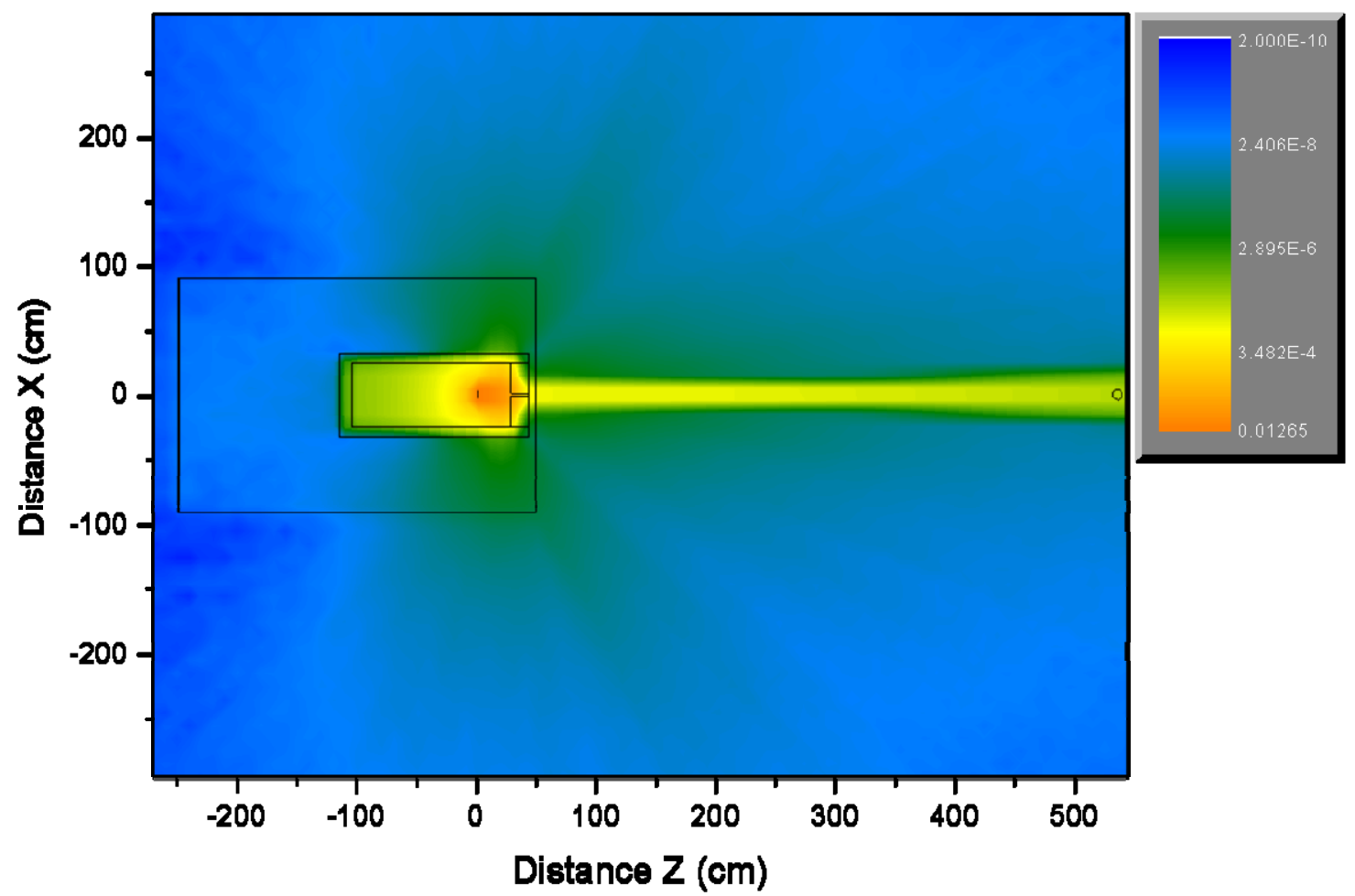

Figure 28. Gamma ray fluence per source electron $\left(\mathrm{cm}^{-2}\right)$ over horizontal planes at an elevation of $\mathbf{0} \mathbf{c m}$.

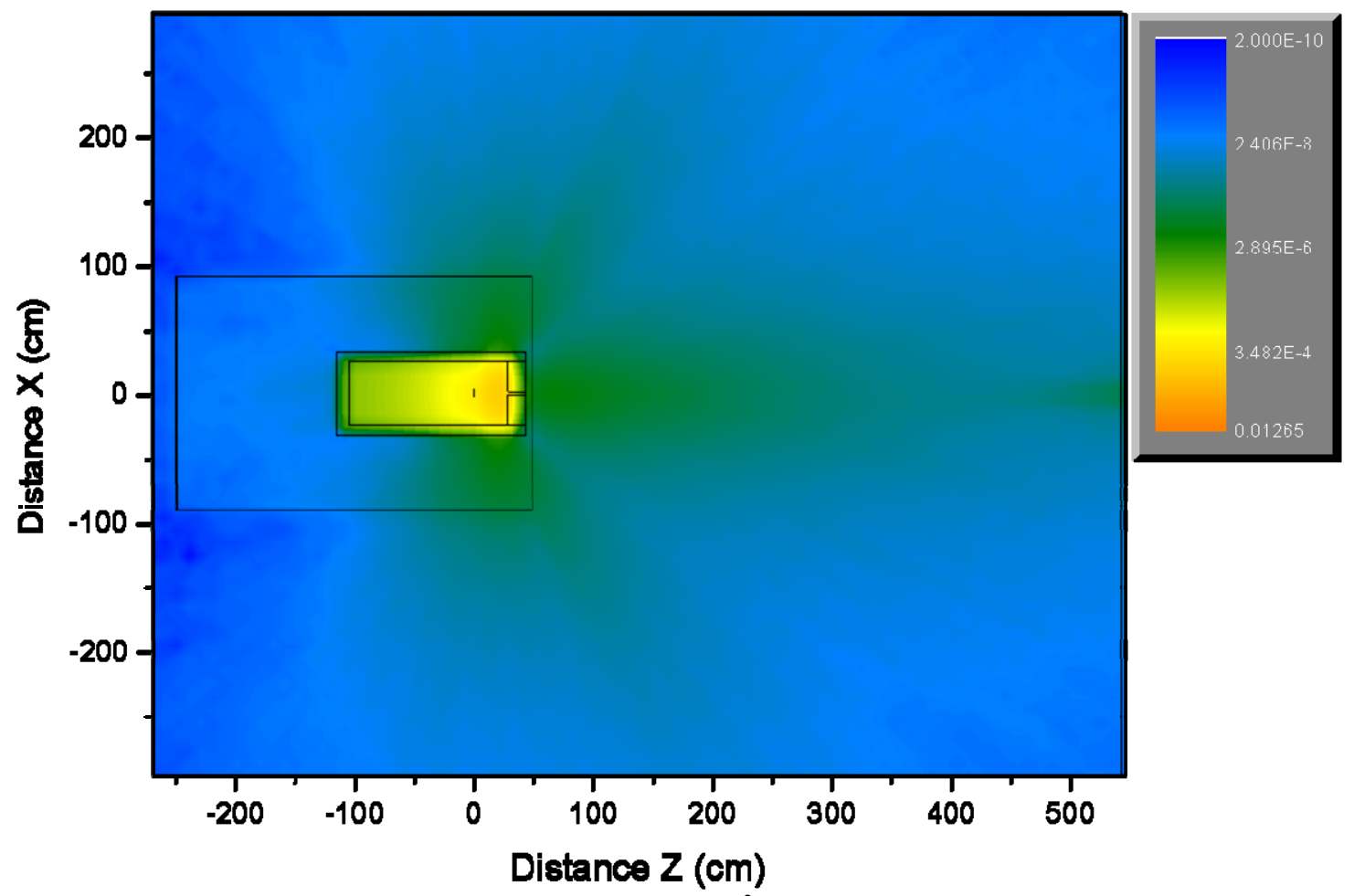

Figure 29. Gamma ray fluence per source electron $\left(\mathrm{cm}^{-2}\right)$ over horizontal planes at an elevation of $-20 \mathrm{~cm}$. 


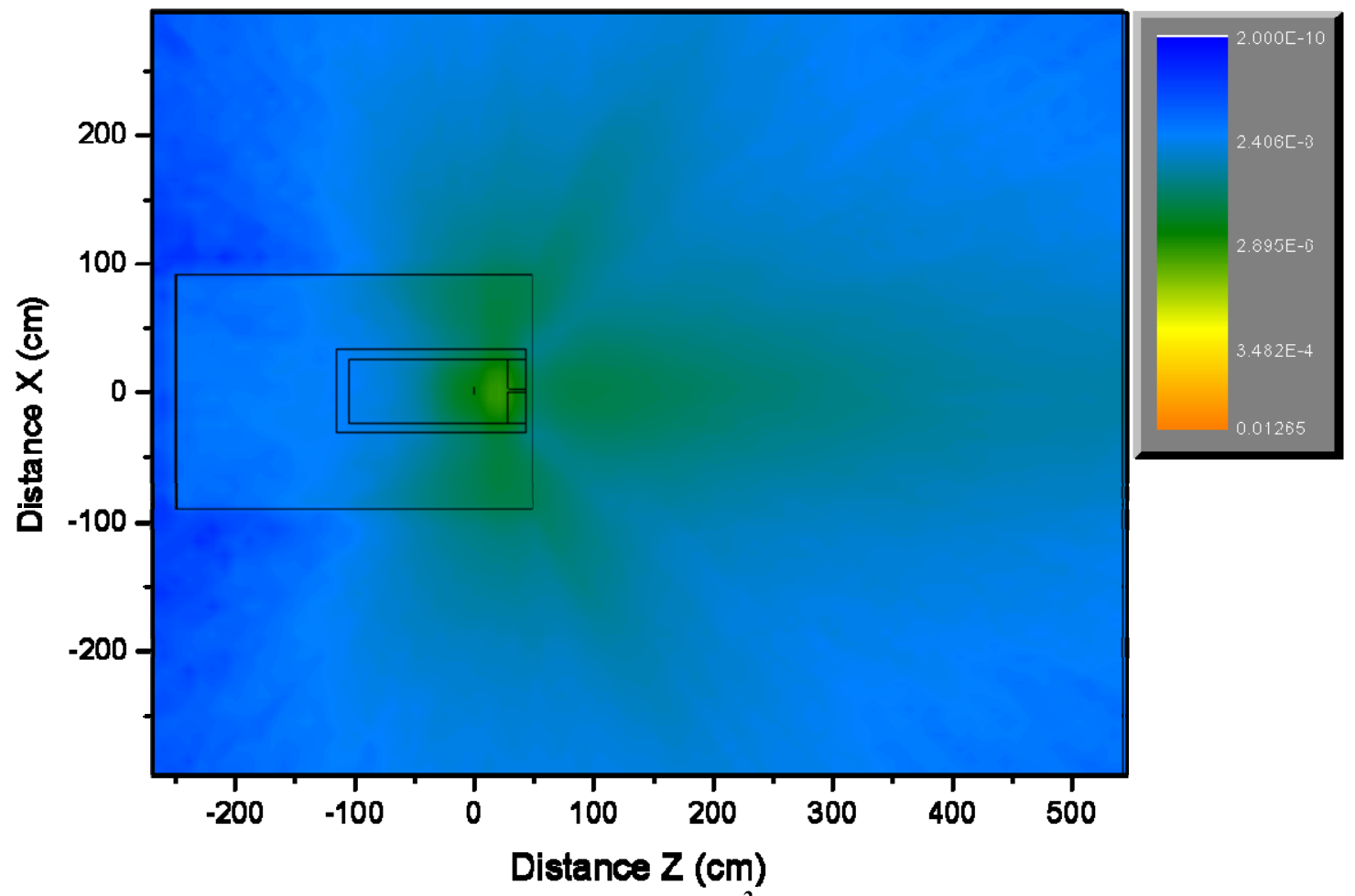

Figure 30. Gamma ray fluence per source electron $\left(\mathrm{cm}^{-2}\right)$ over horizontal planes at an elevation of $-37.62 \mathrm{~cm}$.

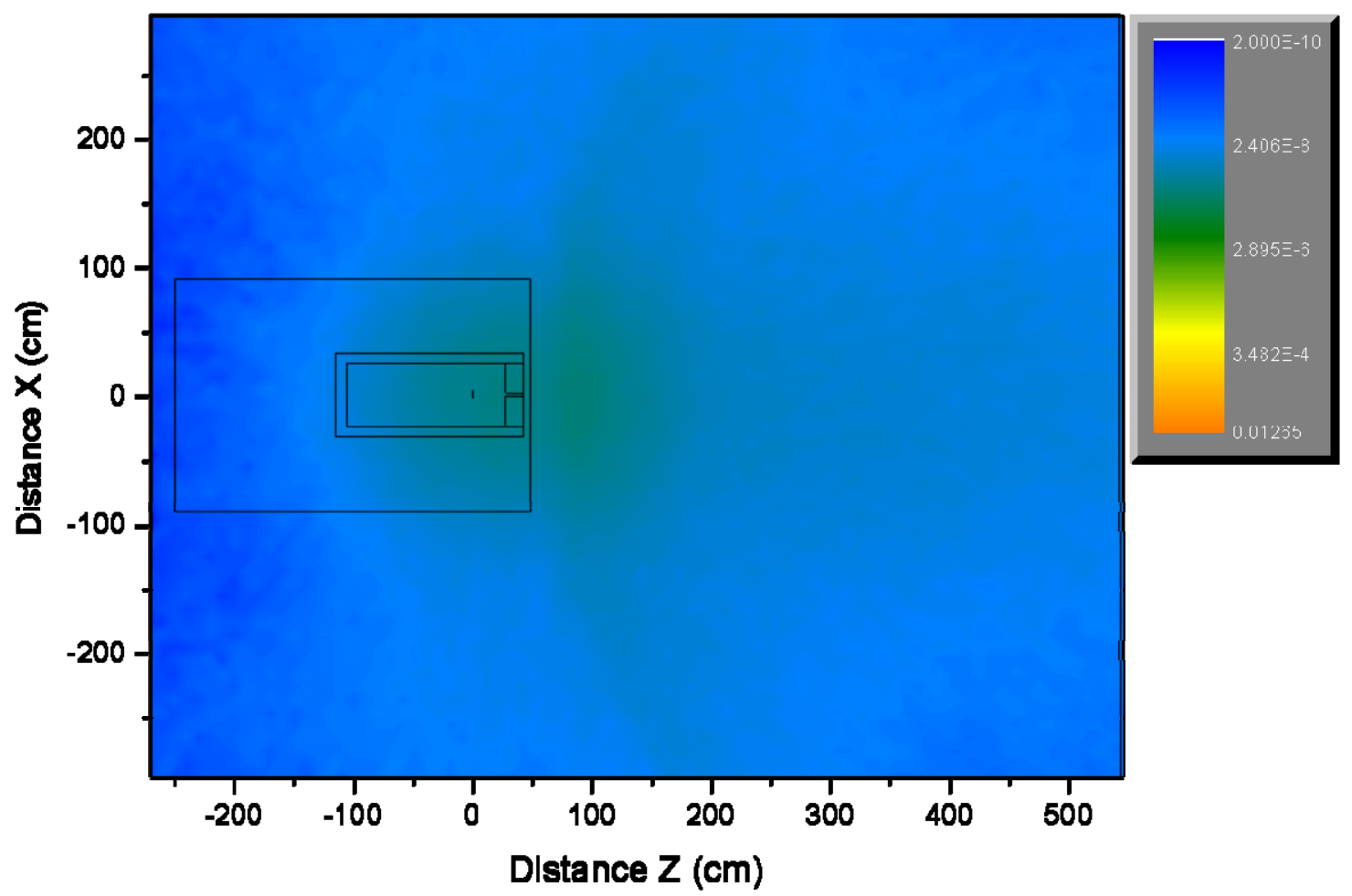

Figure 31. Gamma ray fluence per source electron $\left(\mathrm{cm}^{-2}\right)$ over horizontal planes at an elevation of $-146 \mathrm{~cm}$. 


\section{TIME AND ENERGY DEPENDENCE OF NEUTRON AND PHOTON FLUX}

The MCNPX code ver. 2.5 was used to simulate the number of neutrons that cross the vehicle walls, their energy distribution, as well as their flight time to the vehicle walls. In this section, all results are integrated over the entire surface of the walls. In order to speed up the simulations, appropriate energy cutoffs were again applied. Specifically, an energy cutoff of $4 \mathrm{MeV}$ was used for electrons and gamma rays, while a $10-\mathrm{keV}$ cutoff was applied to the neutrons.

Figure 32 through Figure 34 show the number of neutrons crossing one of the outer walls located vertically along the electron beamline. Only neutrons moving in the outward direction are tallied in these results. The first neutrons created in the converter, the LINAC shielding, or the collimator begin to arrive approximately $20 \mathrm{~ns}$ after initial electron start. Prior to this time, a relatively small number of neutrons are observed. These neutrons arrive at small time delays because of their very large energy, or because they were created directly in the wall by highenergy gamma rays. After $20 \mathrm{~ns}$, the number of neutrons that cross the wall gradually increases, while the peaks of the distributions shift to lower energies. This is due to the arrival of the neutrons, which scattered before reaching the wall. Above $75 \mathrm{~ns}$, the distributions consist of multiple peaks due to multiple neutron scatterings before they reach the wall. The number of neutrons (normalized per ns) decreases approximately by a factor of 70 between the time bins 90 and $95 \mathrm{~ns}$ and 7 and $8 \mu$ s (this bin is not shown).

Similar results are shown in Figure 35 through Figure 37 for the wall located vertically, perpendicular to the beamline in the forward direction. However, this wall is closer to the center of the coordinate system by approximately a factor of two when compared to the side wall discussed earlier. This is reflected in earlier neutron arrival times.

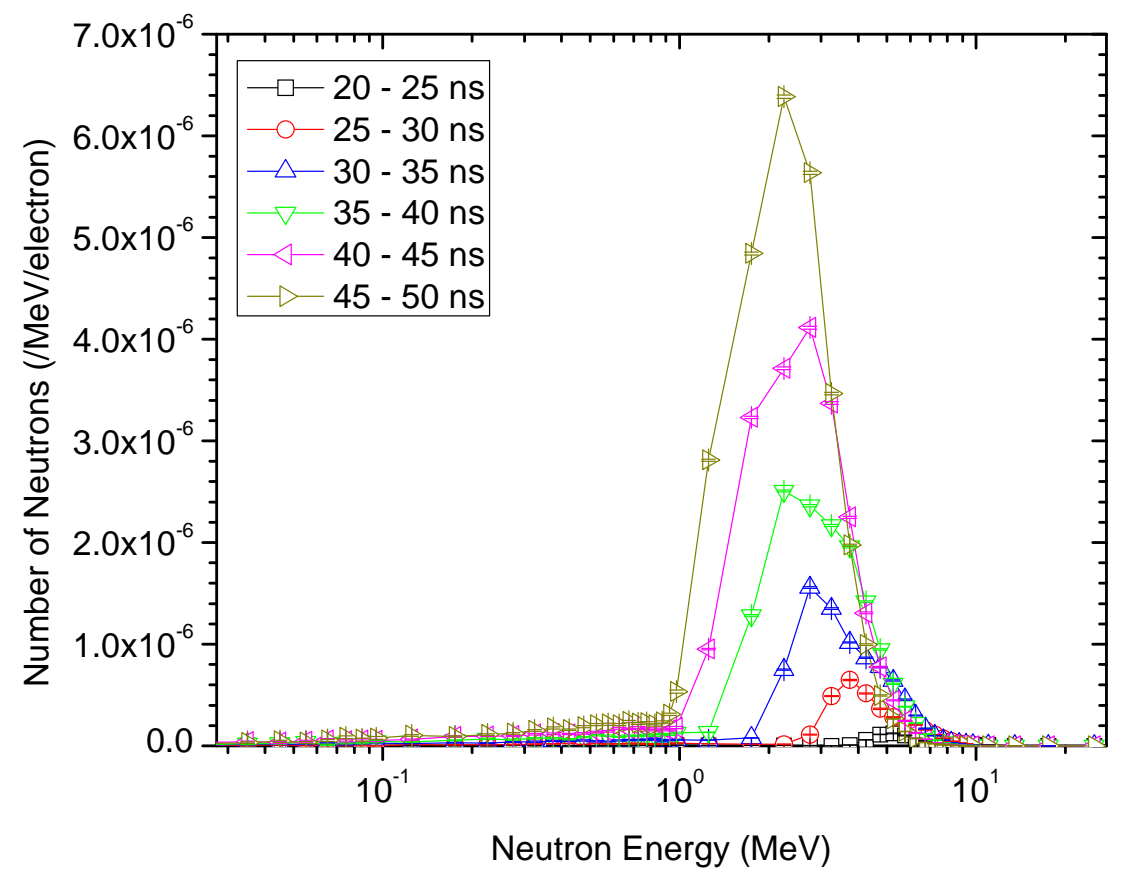

Figure 32. Number of neutrons crossing the vehicle wall located vertically along the beamline for times between 20 and $50 \mathrm{~ns}$. 


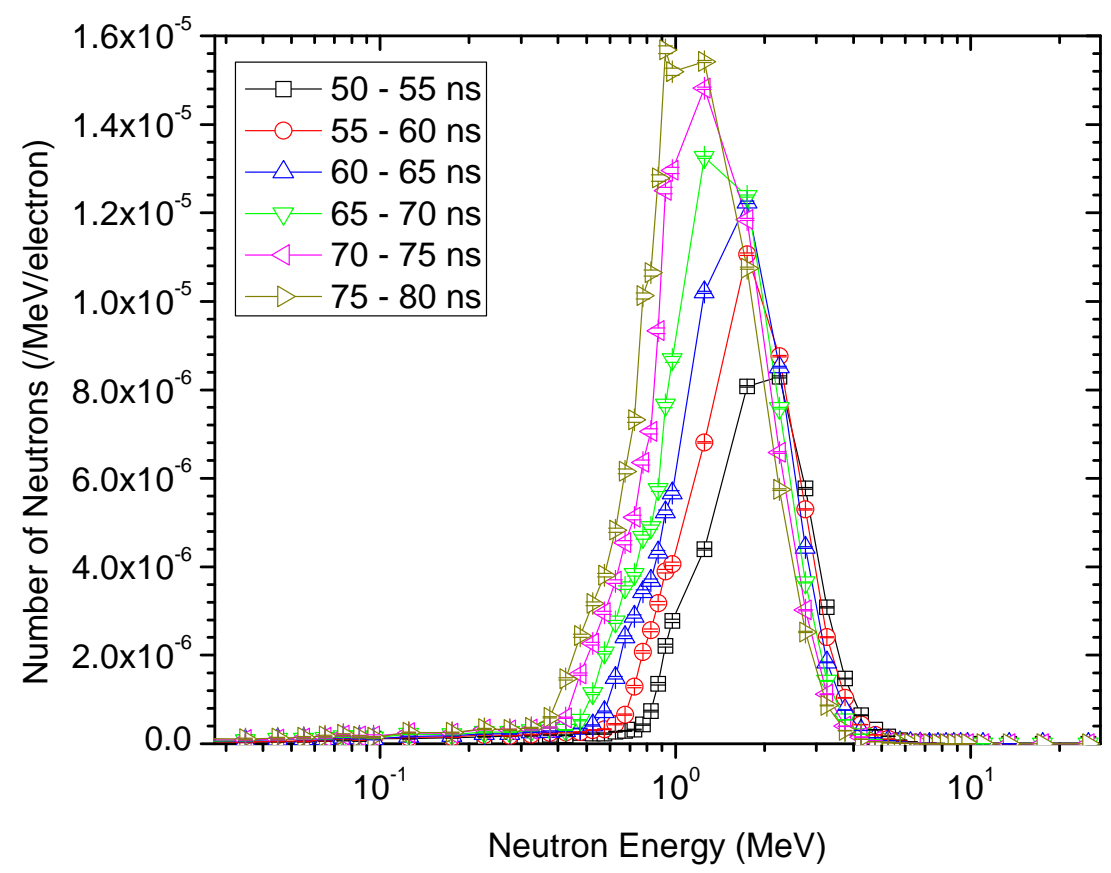

Figure 33. Number of neutrons crossing the vehicle wall located vertically along the beamline between 50 and 80 ns.

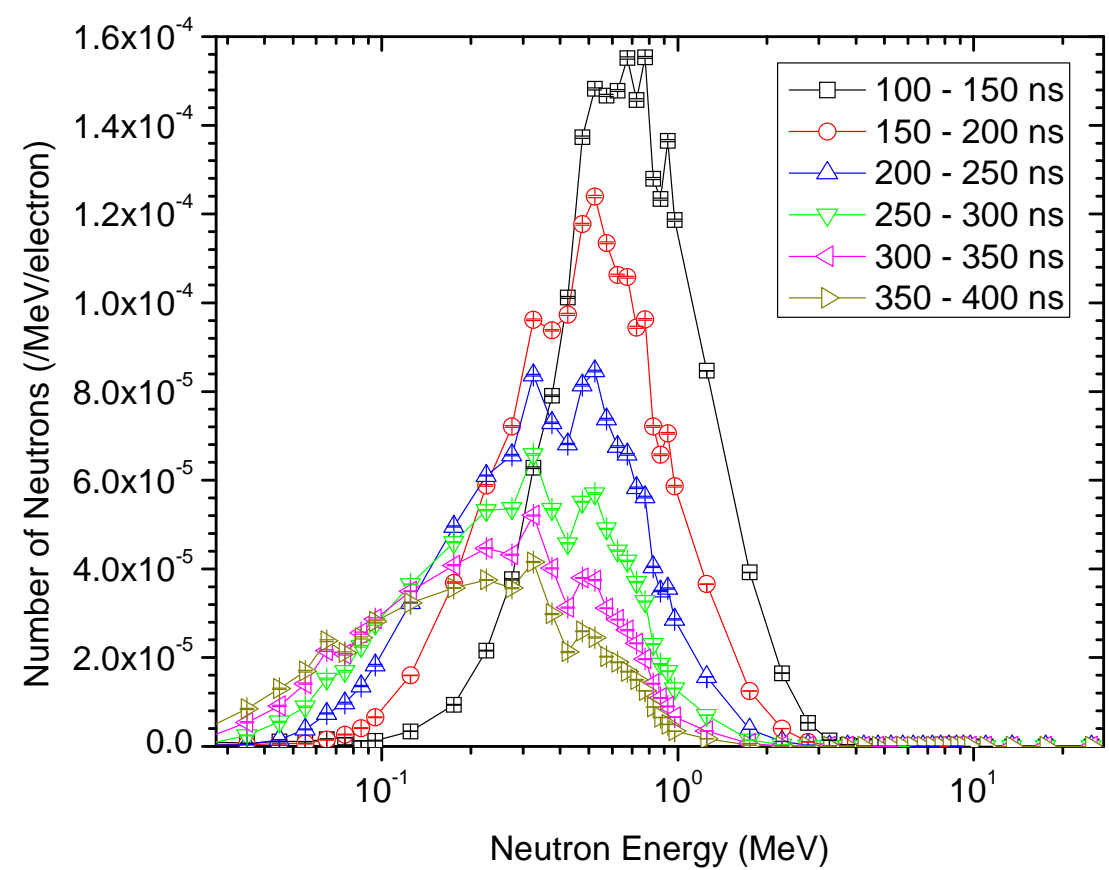

Figure 34. Number of neutrons crossing the vehicle wall located vertically along the beamline between 100 and $400 \mathrm{~ns}$. 


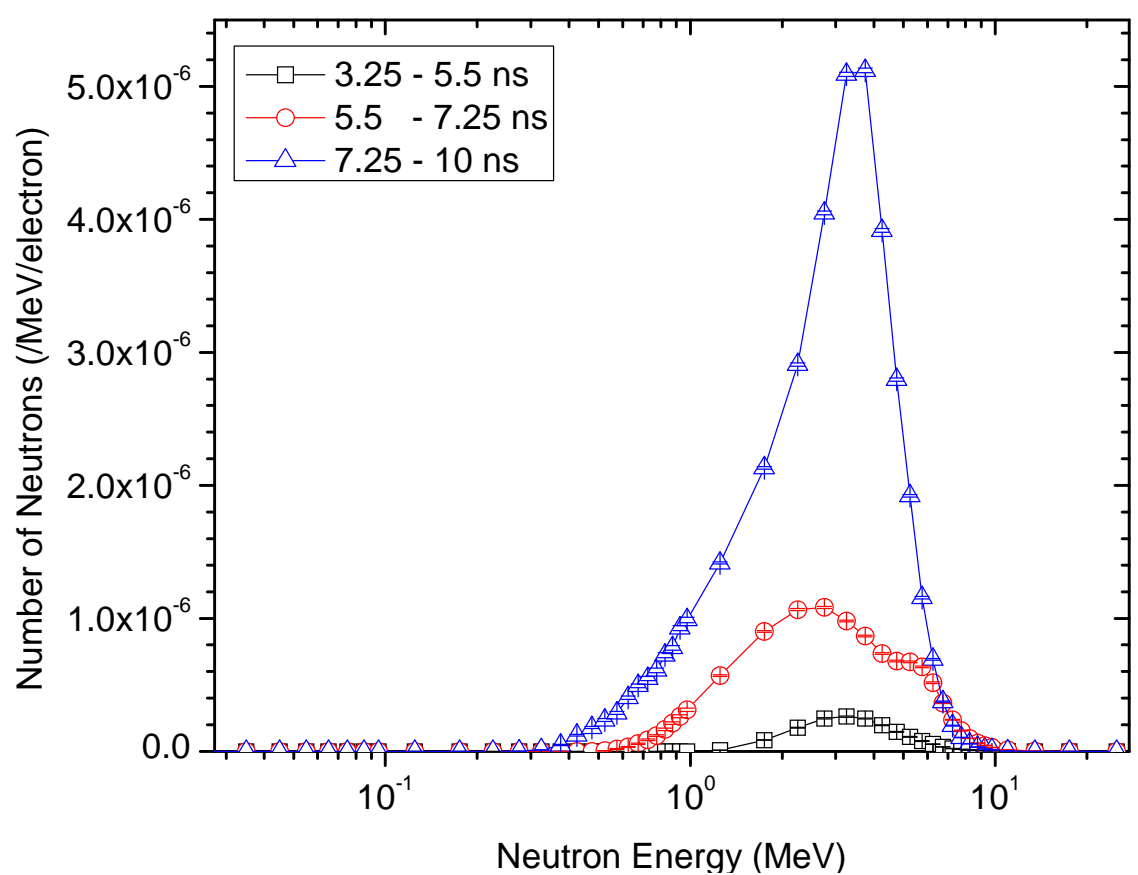

Figure 35. Number of neutrons crossing the vehicle wall located vertically, perpendicular to the beamline in the forward direction, for times between 3.25 and $10 \mathrm{~ns}$.

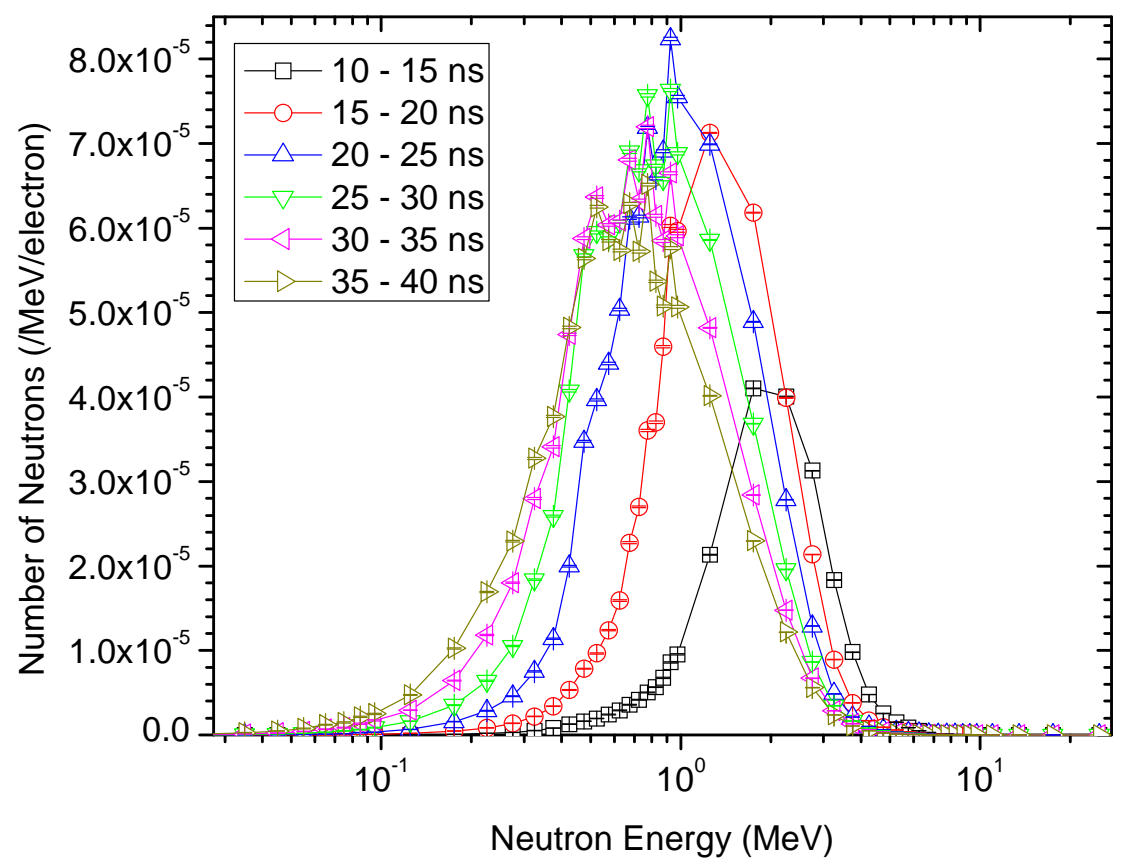

Figure 36. Number of neutrons crossing the vehicle wall located vertically, perpendicular to the beamline in the forward direction, for times between 10 and $40 \mathrm{~ns}$. 


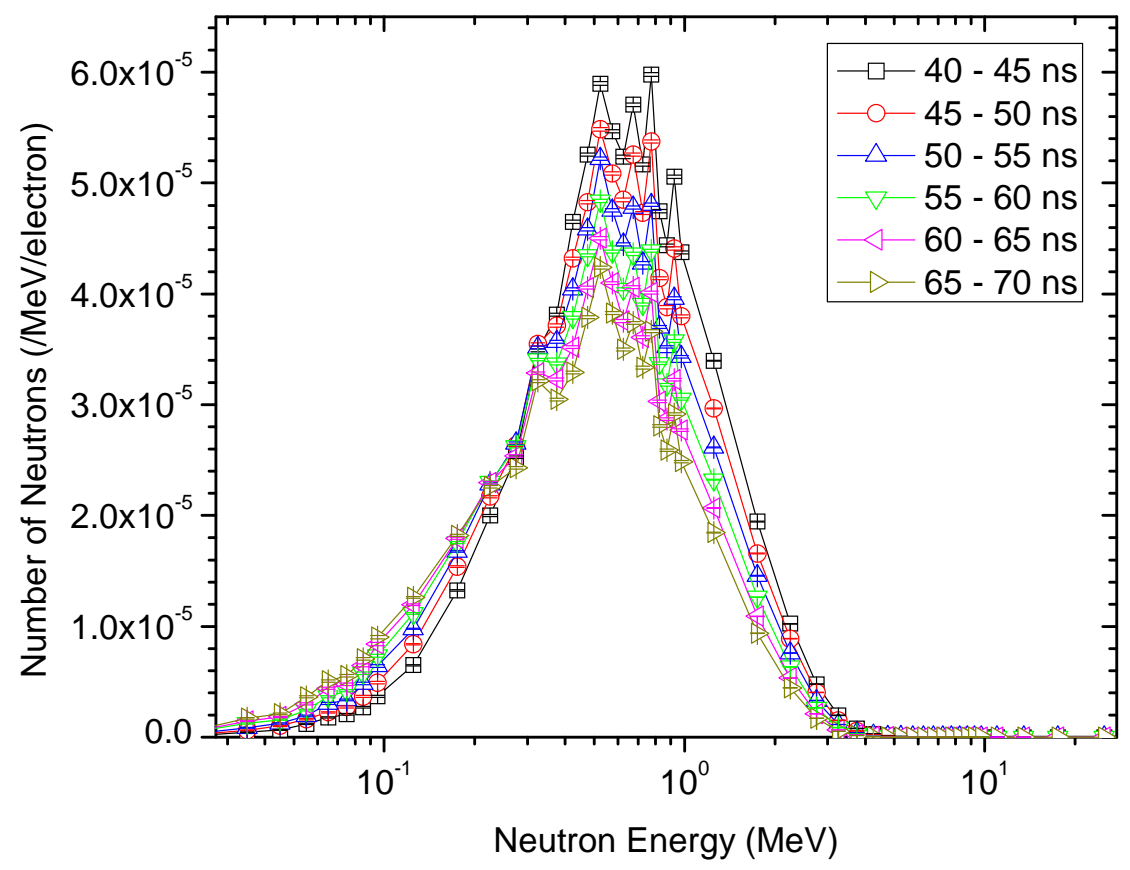

Figure 37. Number of neutrons crossing the vehicle wall located vertically, perpendicular to the beamline in the forward direction, for times between 40 and 70 ns.

Therefore, in order to possibly detect the delayed neutrons, the detectors should be active only after all prompt neutrons have scattered out of the system. These results show this time to be greater than $5 \mu$ s after the accelerator pulse.

\section{PHOTONUCLEAR SOURCE CHARACTERIZATION}

\subsection{Photoneutron Production-Prompt Neutrons Only}

A two-step method was used to simulate the photonuclear reactions in a given target. First, a modified version of MCNPX was used to calculate the location of photonuclear events and to write a source file to be read by MCNP-PoliMi. In the second step MCNP-PoliMi reads this source file, which contains all the necessary information to start a particle history, and performs the full transport of each photonuclear history. In this work, the MCNPX/MCNP-PoliMi codes were used to model the prompt photoneutron production in two targets located $5 \mathrm{~m}$ from the collimator:

1. A 5-kg sphere of depleted uranium (DU): $100 \%{ }^{238} \mathrm{U}$ )

2. A 5 -kg sphere of highly enriched uranium (HEU): $98 \%{ }^{235} \mathrm{U}$ and $2 \%{ }^{238} \mathrm{U}$ )

\subsection{DU Target}

The prompt photoneutron flux was calculated at several different locations, but the results at six planes are included here. The planes are located at $5 \mathrm{~m}$ from the collimator, $3 \mathrm{~m}$ from the collimator, and at the back of the vehicle in which the accelerator is located (approximately 
$6.5 \mathrm{~cm}$ from the collimator). Three other planes were also plotted that show the prompt photoneutron flux along the side of the vehicle. The first of these planes along the side of the vehicle is located $4 \mathrm{~cm}$ in front of the converter plane, the second is $56 \mathrm{~cm}$ behind the converter, and the third is $96 \mathrm{~cm}$ behind the converter. All of the tallies upstream and downstream of the collimator were perpendicular to the beam axis (shown in Figure 2). The tally at each plane was segmented into several rectangular regions. All of the tally regions are $10 \times 10 \mathrm{~cm}^{2}$ squares. However, the tallies do not extend inside the vehicle, so the tallies directly adjacent to the vehicle have been truncated. The DU prompt photoneutron fluxes at these six locations can be seen in Figure 38 through Figure 43. The relative errors for Figure 38 through Figure 43 are less than 0.05 .

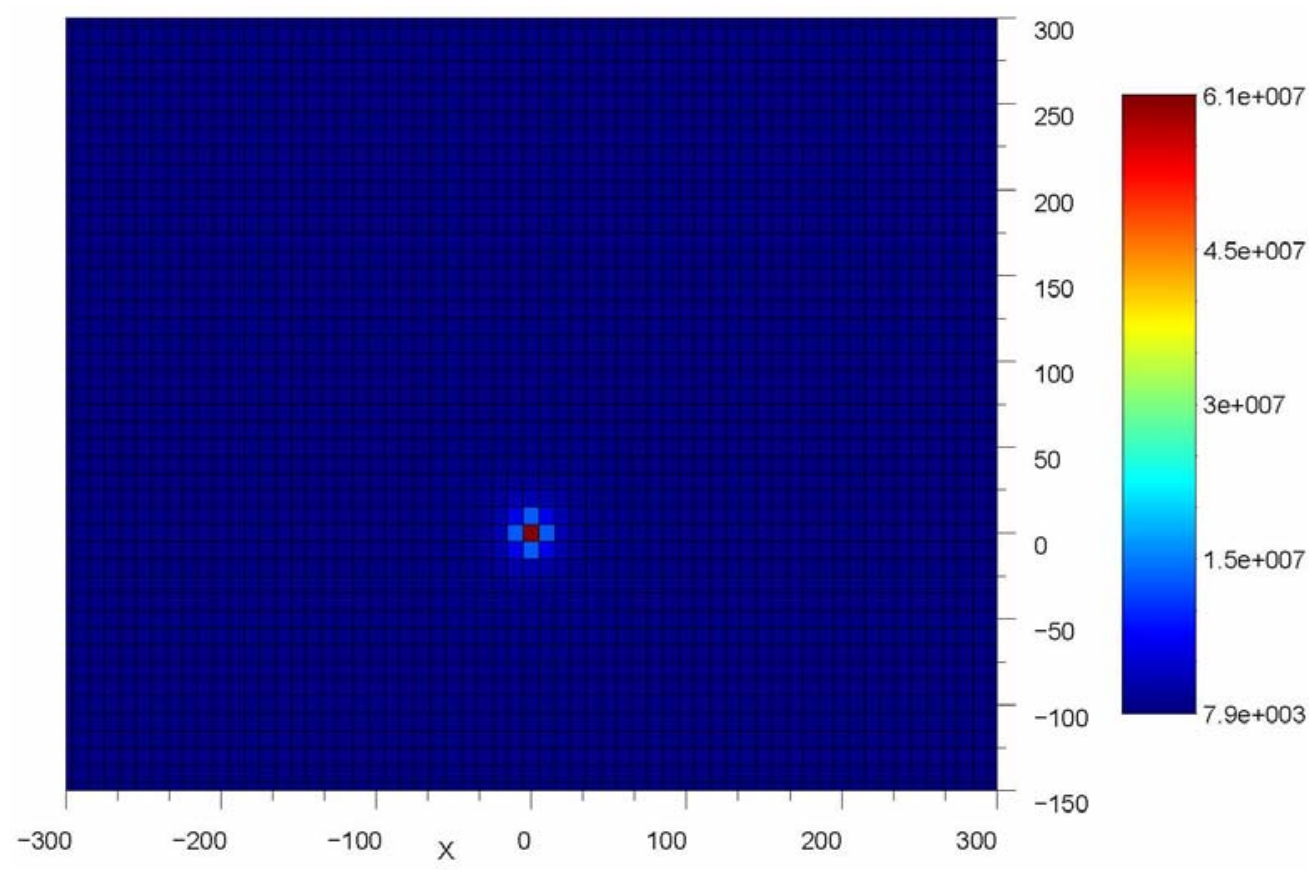

Figure 38. Depleted uranium prompt photoneutron flux $\left(\mathrm{cm}^{-2} \mathrm{sec}^{-1}\right) 5 \mathrm{~m}$ from exit of the collimator (at target). 


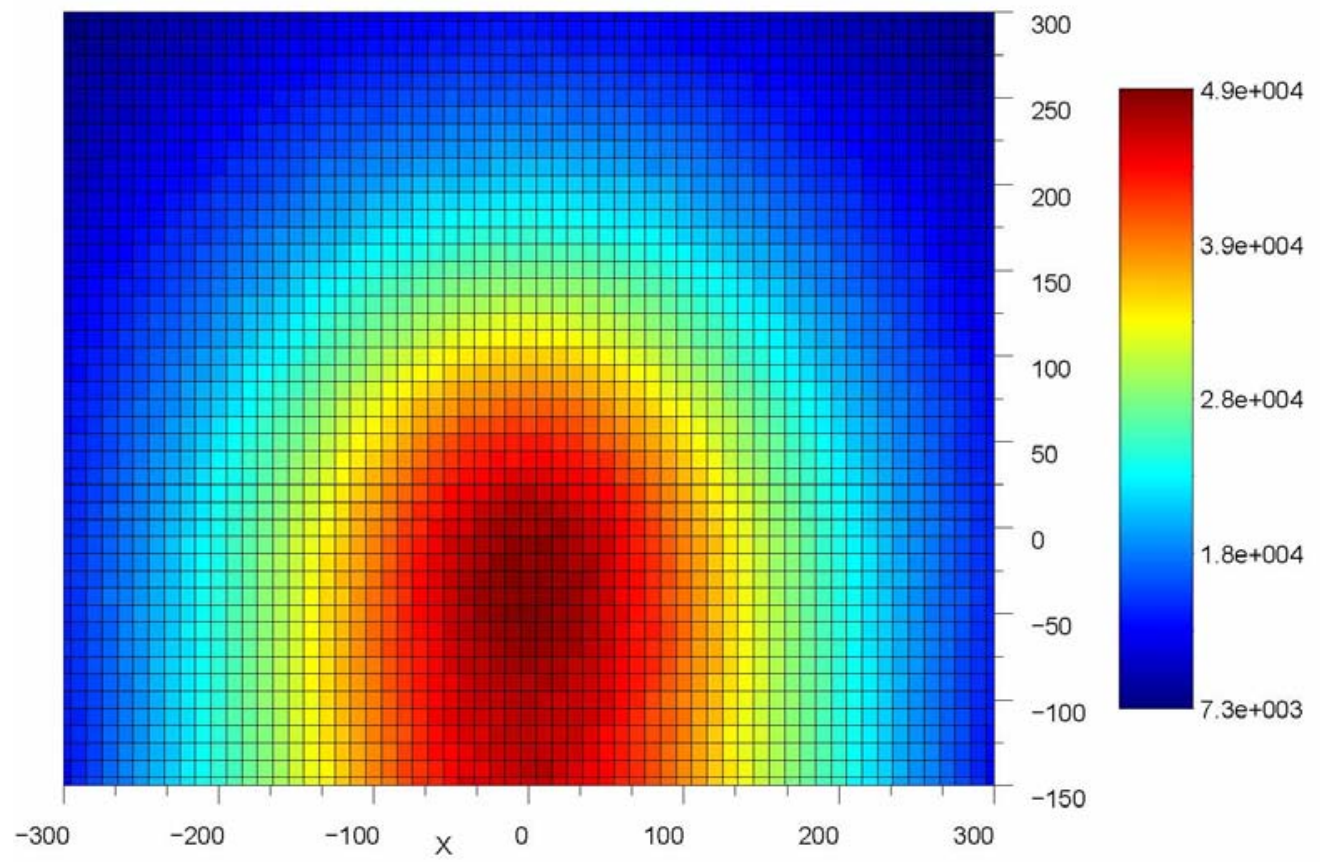

Figure 39. Depleted uranium prompt photoneutron flux $\left(\mathrm{cm}^{-2} \mathrm{sec}^{-1}\right) 3 \mathrm{~m}$ from exit of the collimator.

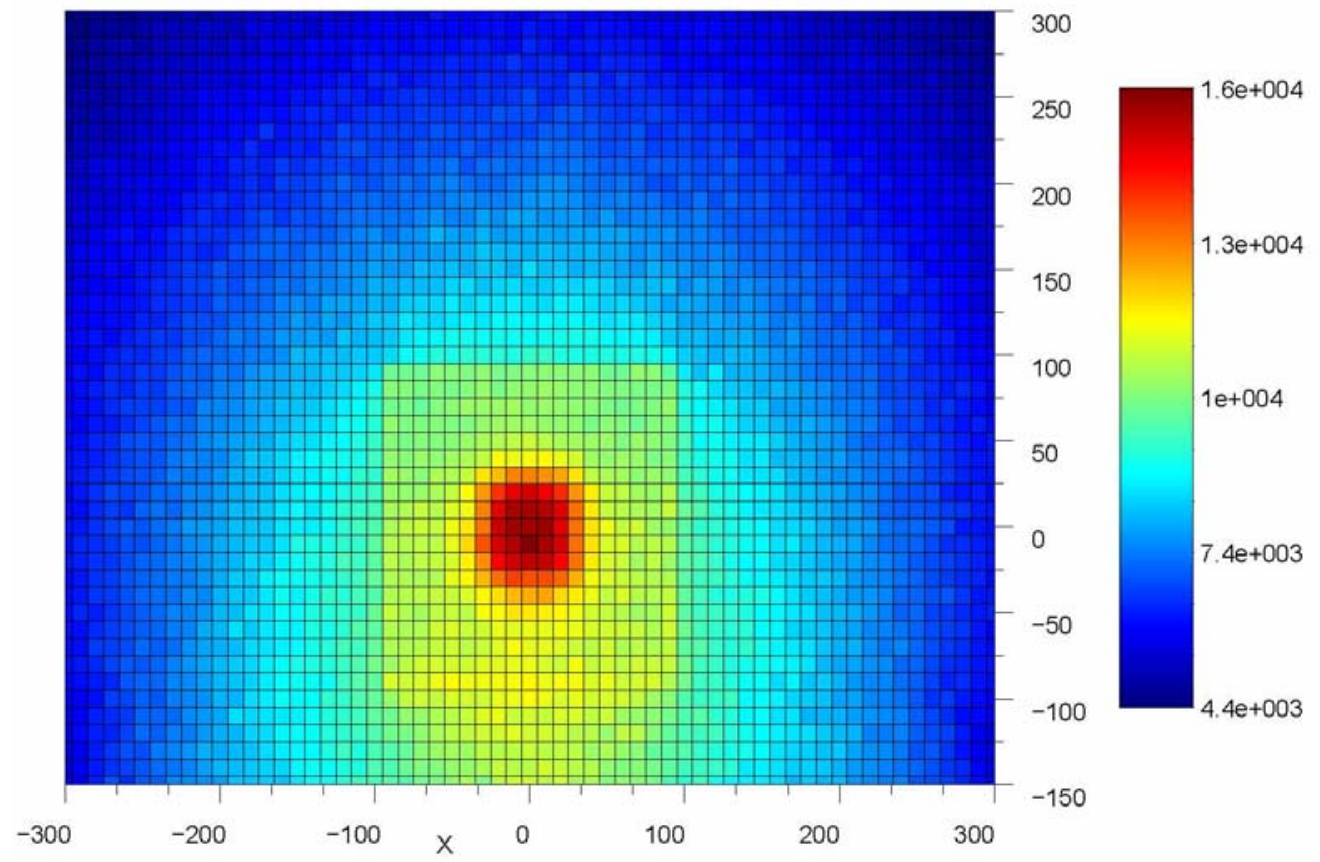

Figure 40. Depleted uranium prompt photoneutron flux $\left(\mathrm{cm}^{-2} \mathrm{sec}^{-1}\right) 6.5 \mathrm{~cm}$ from exit of the collimator. 


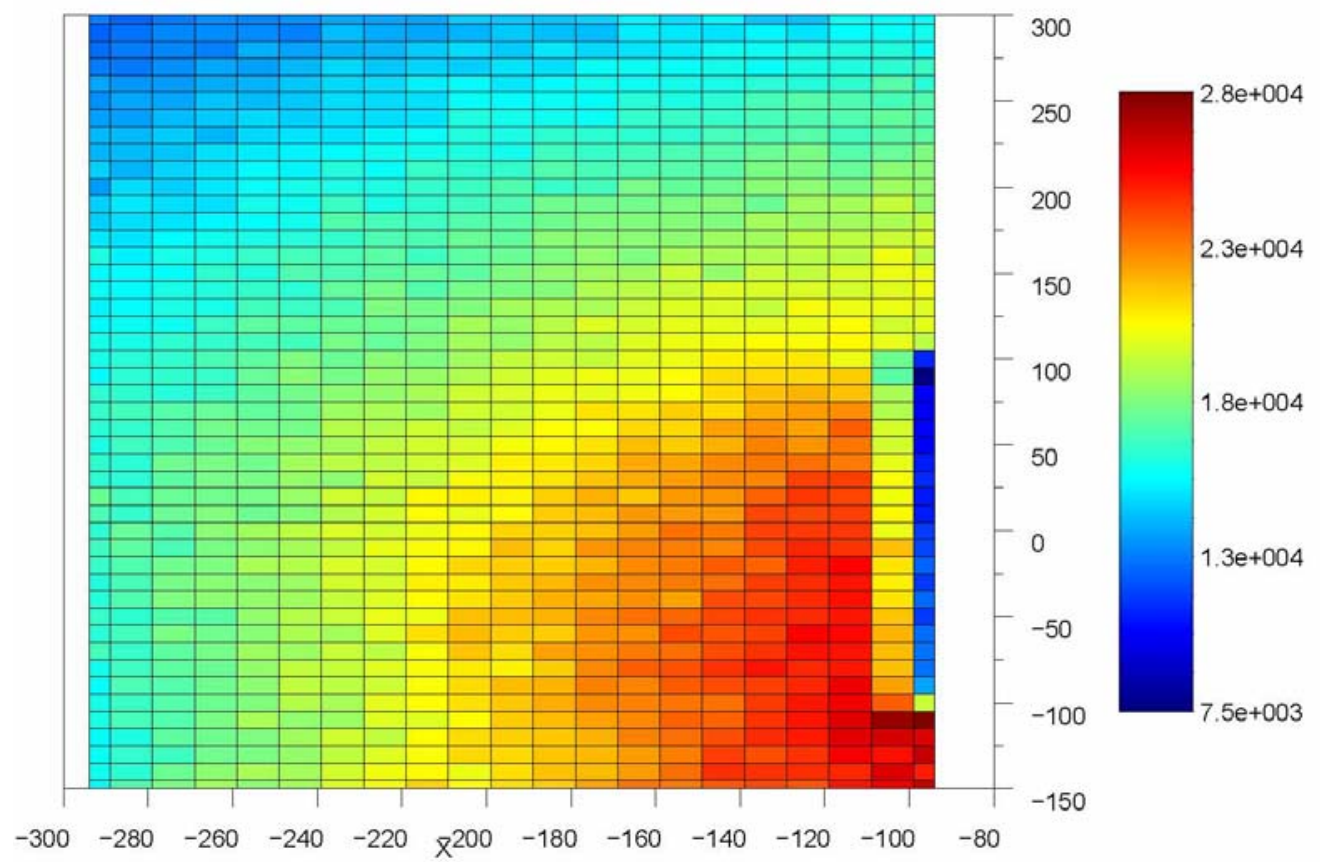

Figure 41. Depleted uranium prompt photoneutron flux $\left(\mathrm{cm}^{-2} \mathrm{sec}^{-1}\right)$ on the side of the vehicle $4 \mathrm{~cm}$ downstream from the converter. Flux on the other side of the vehicle is symmetric.

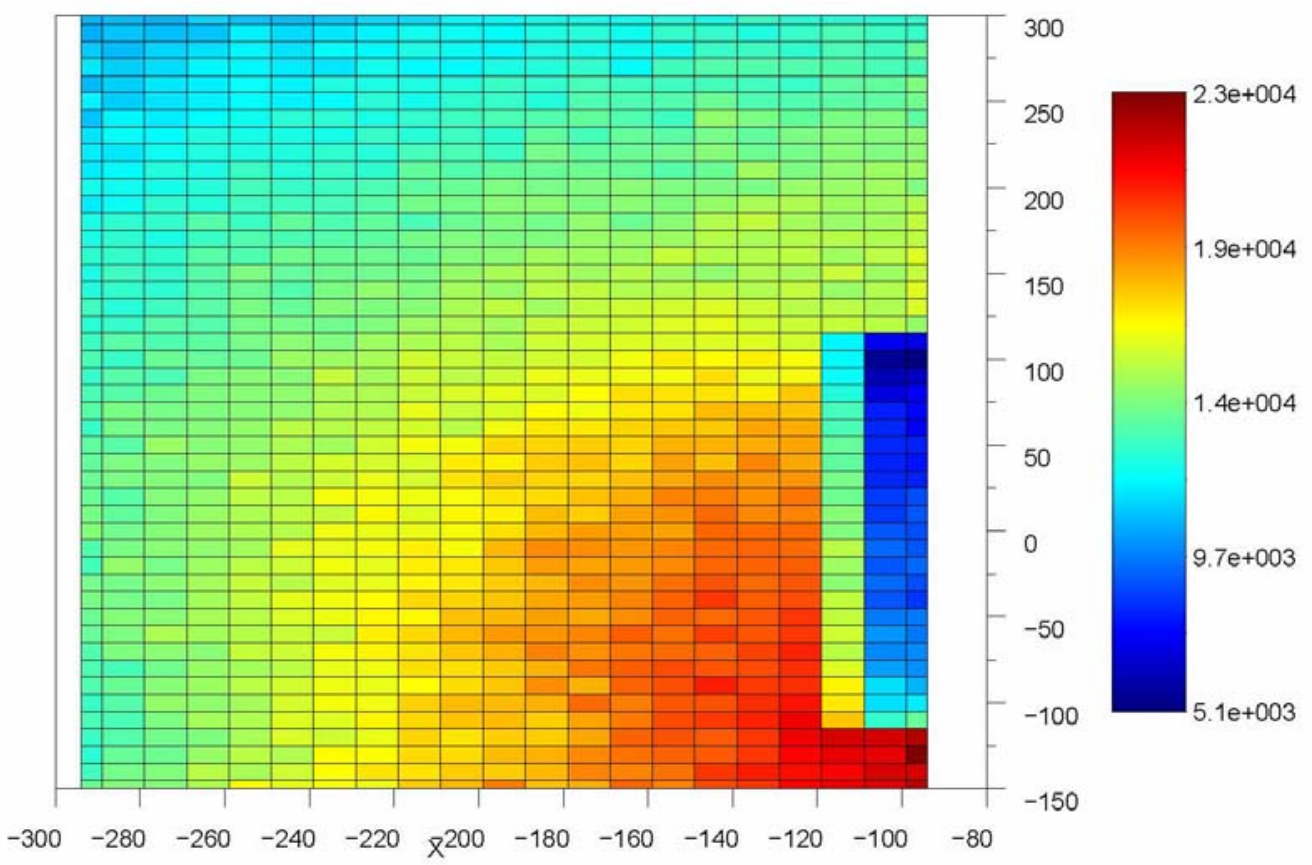

Figure 42. Depleted uranium prompt photoneutron flux $\left(\mathrm{cm}^{-2} \mathrm{sec}^{-1}\right)$ on the side of the vehicle $56 \mathrm{~cm}$ upstream from the converter. Flux on the other side of the vehicle is symmetric. 


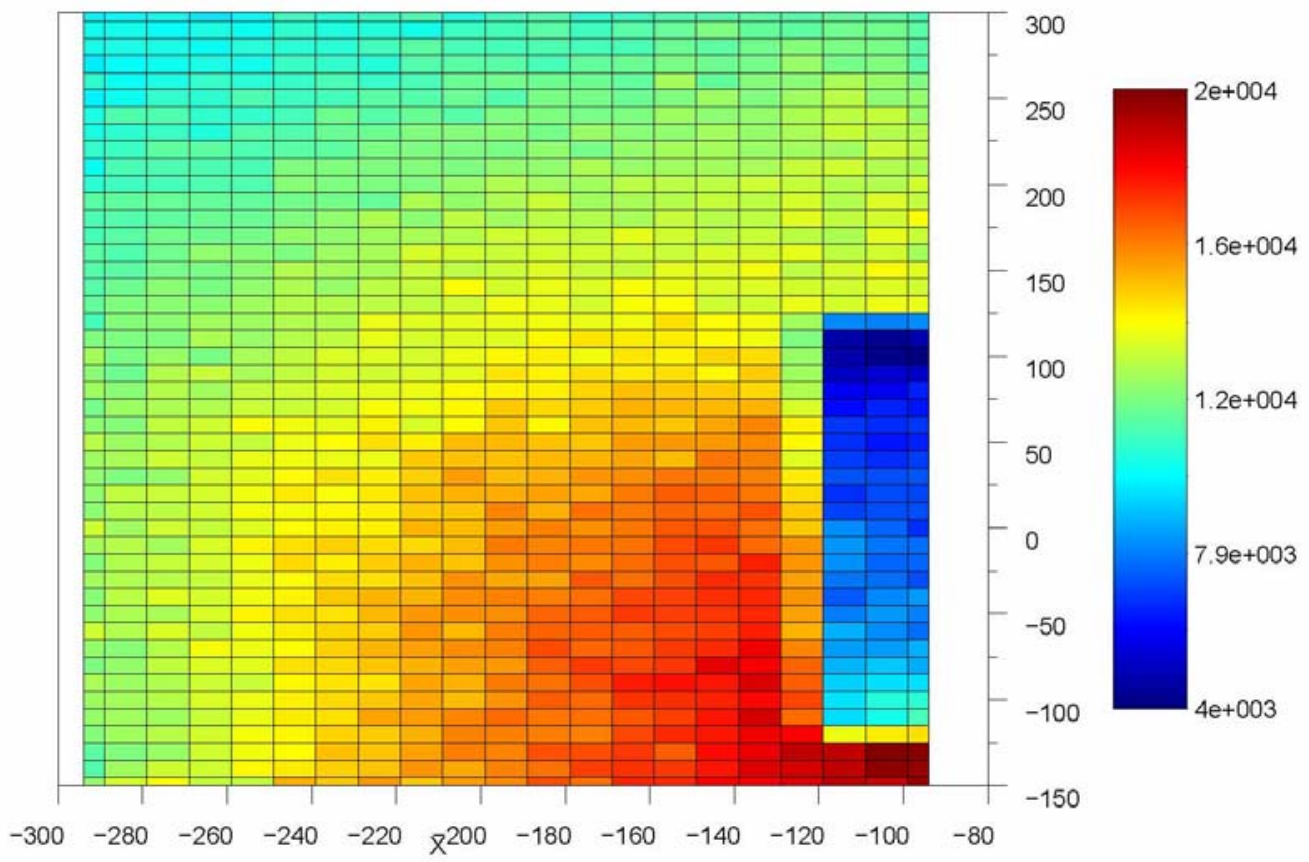

Figure 43. Depleted uranium prompt photoneutron flux $\left(\mathrm{cm}^{-2} \mathrm{sec}^{-1}\right)$ on the side of the vehicle $96 \mathrm{~cm}$ upstream from the converter. Flux on the other side of the vehicle is symmetric.

One interesting observation in Figure 41 through Figure 43 is that the side of the vehicle causes a "shadow." The side of the vehicle is located at about $-95 \mathrm{~cm}$ on the $x$-axis and extends from about $-125 \mathrm{~cm}$ up to $125 \mathrm{~cm}$ along the $y$-axis. As one moves from the back of the vehicle toward the front of the vehicle (Figure 41 through Figure 43) the shadow becomes larger as more cells have their line of sight to the target cutoff. In Figure 38 through Figure 43, the effect of the ground reflecting neutrons is apparent.

\subsection{HEU Target}

The highly enriched uranium prompt photoneutron fluxes at the same six locations can be seen below in Figure 44 through Figure 49. The relative errors for Figure 44 through Figure 49 are again all less than 0.05 . 


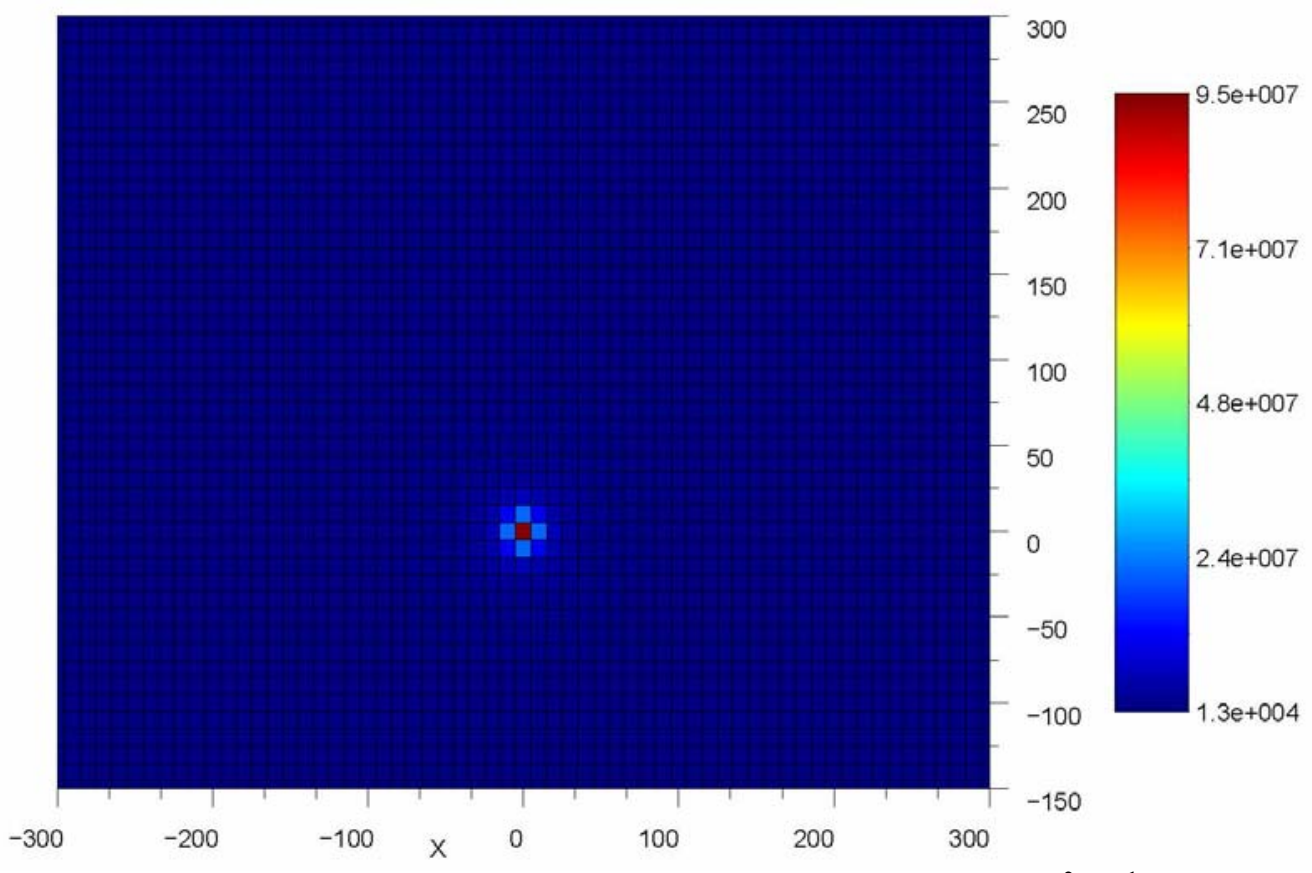

Figure 44. Highly enriched uranium prompt photoneutron flux $\left(\mathrm{cm}^{-2} \mathrm{sec}^{-1}\right) 5 \mathrm{~m}$ from exit of the collimator (at target).

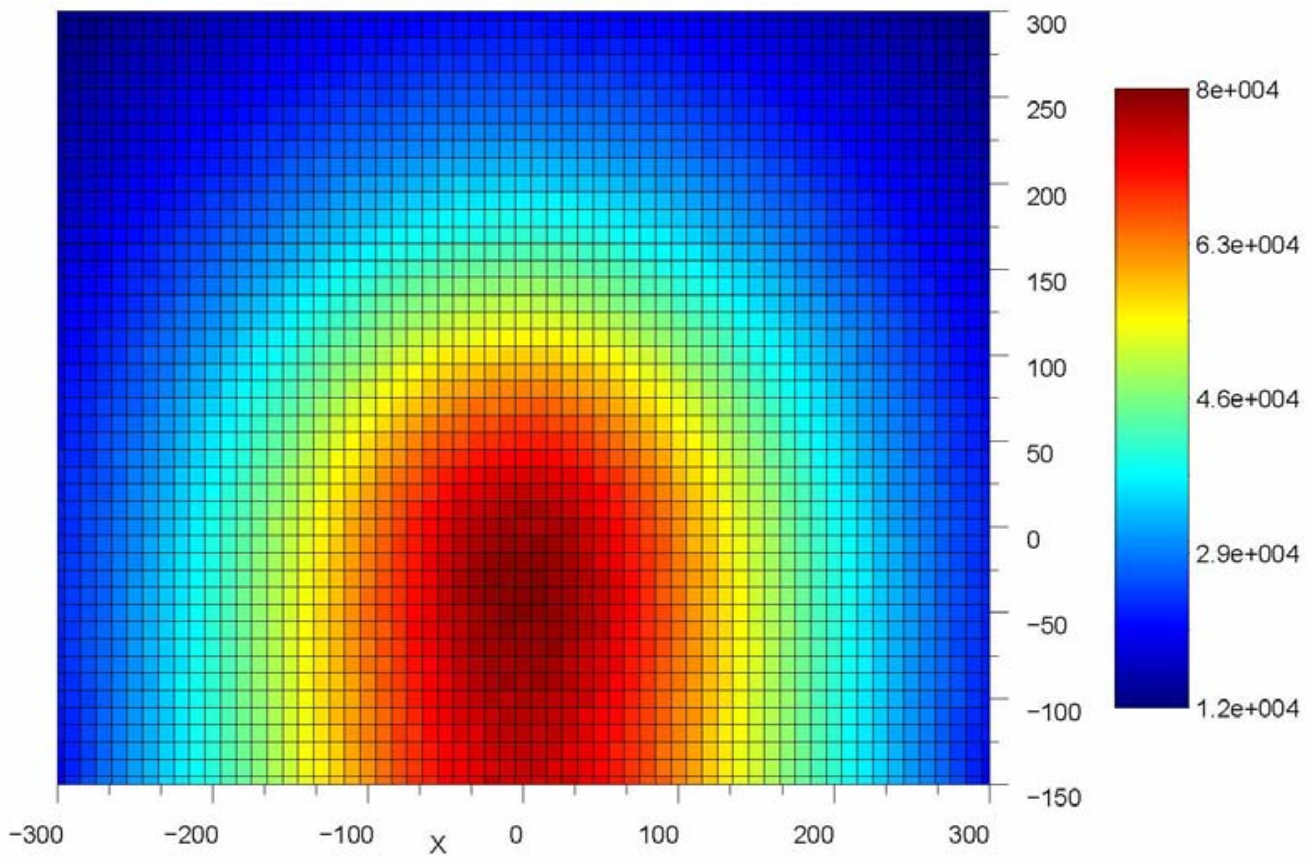

Figure 45. Highly enriched uranium prompt photoneutron flux $\left(\mathrm{cm}^{-2} \mathrm{sec}^{-1}\right) 3 \mathrm{~m}$ from exit of the collimator. 


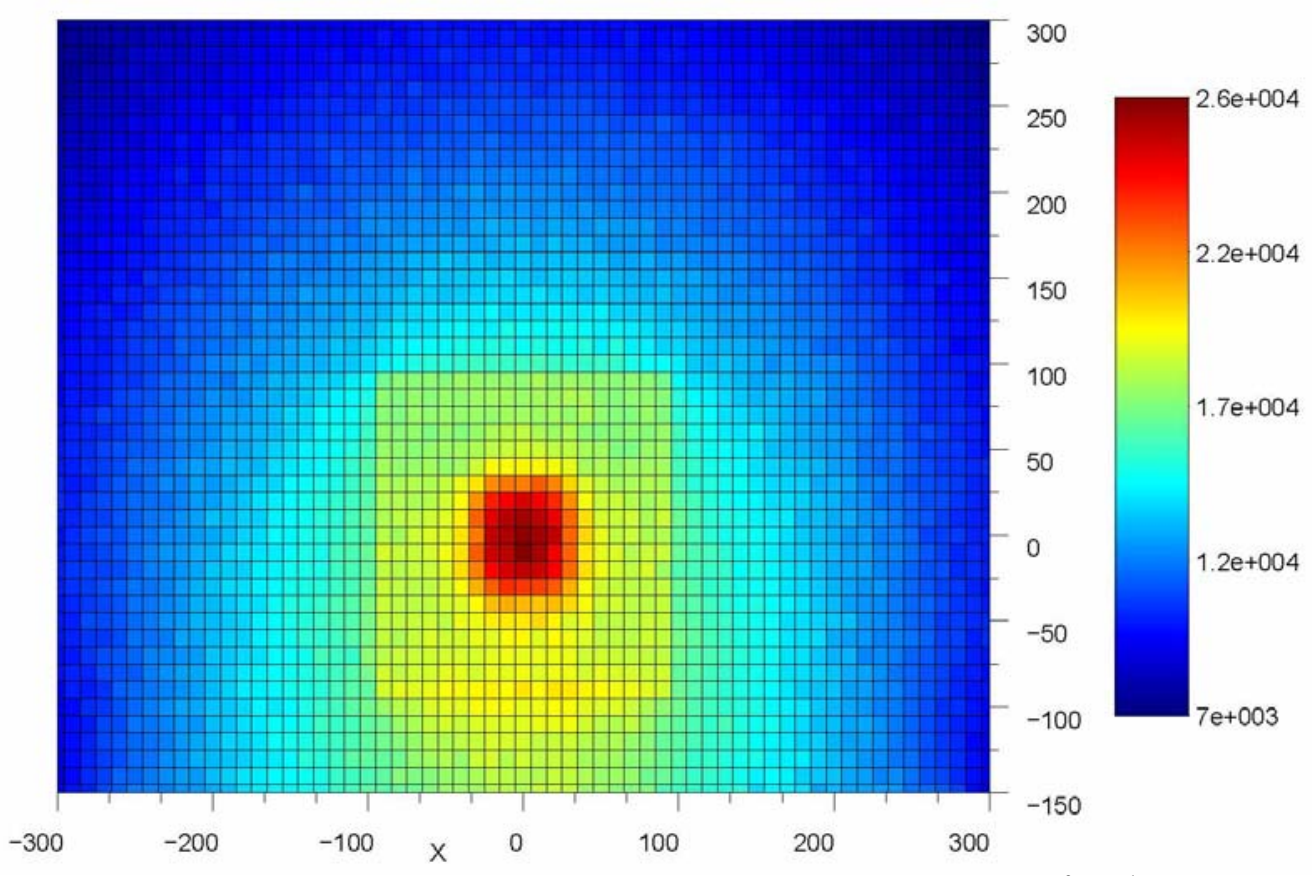

Figure 46. Highly enriched uranium prompt photoneutron flux $\left(\mathrm{cm}^{-2} \mathrm{sec}^{-1}\right) 6.5 \mathrm{~cm}$ from exit of the collimator.

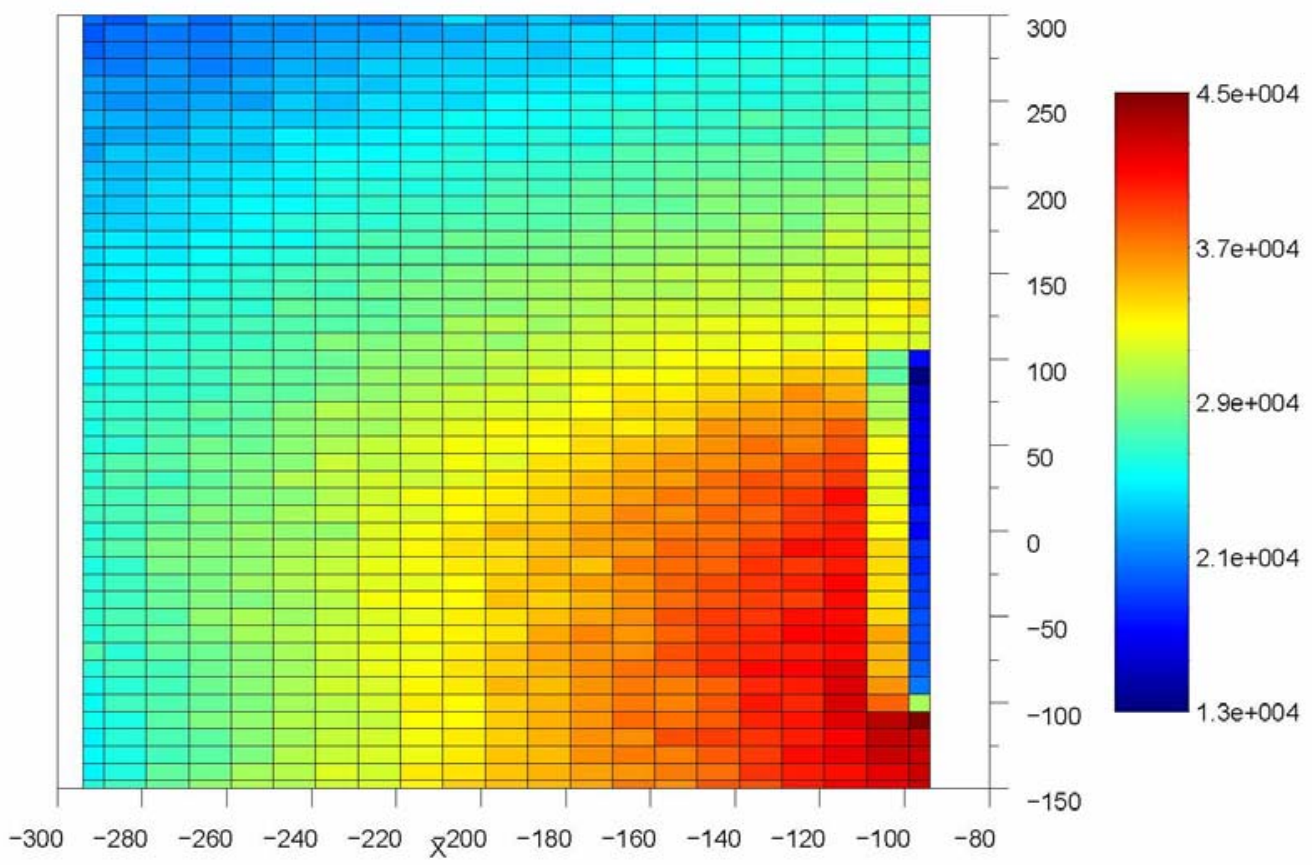

Figure 47. Highly enriched uranium prompt photoneutron flux $\left(\mathrm{cm}^{-2} \mathrm{sec}^{-1}\right)$ along the side of the vehicle $4 \mathbf{c m}$ downstream from the converter. Flux on the other side of the vehicle is symmetric. 


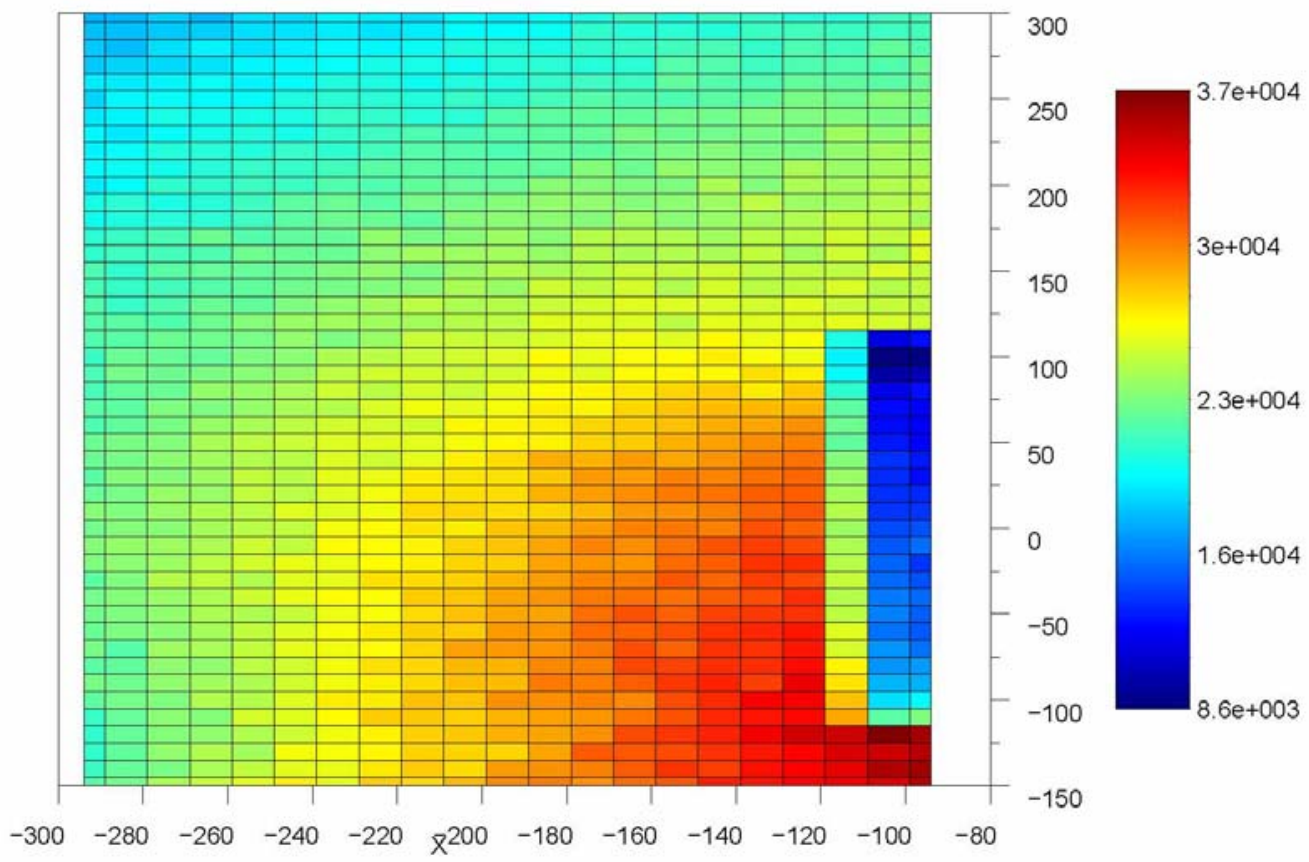

Figure 48. Highly enriched uranium prompt photoneutron flux $\left(\mathrm{cm}^{-2} \mathrm{sec}^{-1}\right)$ along the side of the vehicle $56 \mathrm{~cm}$ upstream from the converter. Flux on the other side of the vehicle is symmetric.

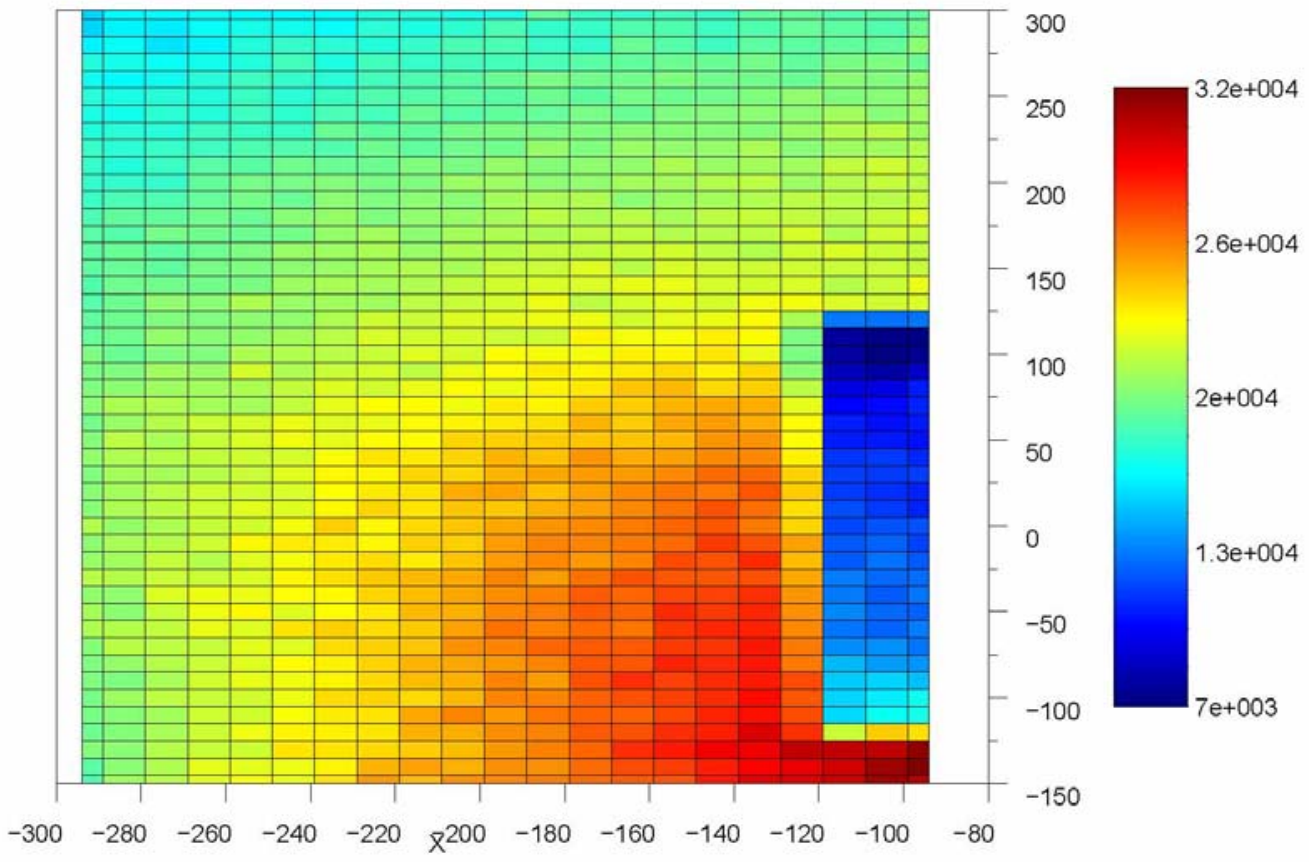

Figure 49. Highly enriched uranium prompt photoneutron flux $\left(\mathrm{cm}^{-2} \mathrm{sec}^{-1}\right)$ along the side of the vehicle $96 \mathrm{~cm}$ upstream from the converter. Flux on the other side of the vehicle is symmetric. 
The primary difference between the response from the HEU target and that of the DU target is the number of neutrons emitted; the flux map is otherwise similar. In fact, the response from the HEU is visually 50 percent greater throughout these results. These flux maps indicate that the detectors should be placed approximately $40 \mathrm{~cm}$ away from the vehicle and $150 \mathrm{~cm}$ above the ground (shown in Figure 48).

\section{DELAYED NEUTRON MODELING}

To approximate the relative contribution of delayed neutrons to the total neutron field, a simple delayed neutron source spectrum model was implemented in the MCNPX model (given in Appendix G). A 400-keV Maxwellian energy spectrum was generated homogeneously within the depleted uranium target and the resulting neutron fluence was tallied in the $x$-z plane at various $y$ values (elevations) throughout the entire geometry. The spectrum, shown in Figure 50, is expressed in units of delayed neutrons per square-centimeter per $\mathrm{MeV}$ for each source-delayed neutron.

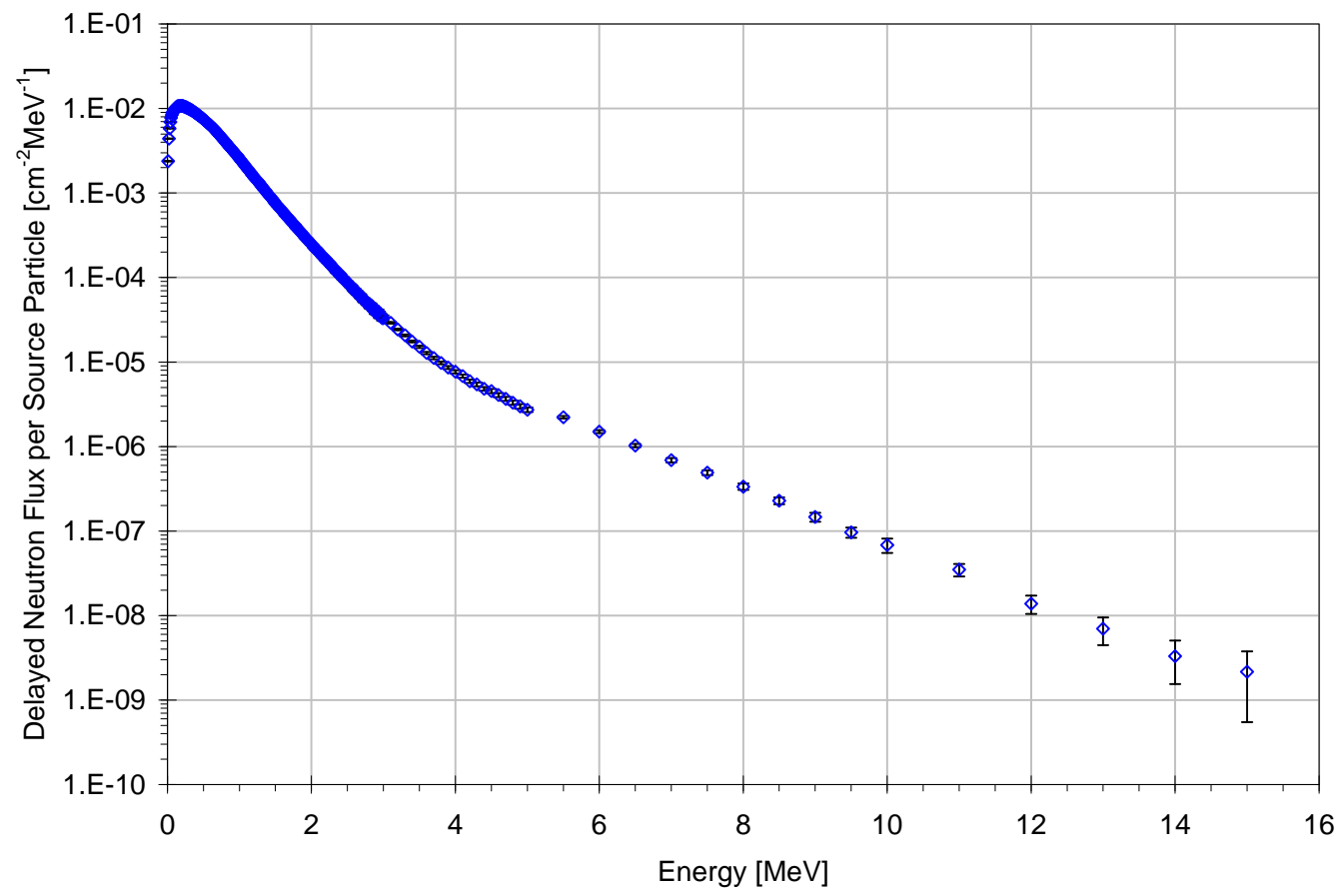

Figure 50. Maxwellian energy spectrum (400 keV) used to approximate the delayed neutron source. The flux is tallied on the surface of the target per source delayed neutron; three-sigma error bars are shown.

Figure 51 shows the results within the $x$-z plane at the geometric midplane $(y=0)$ of the system. The isotropic nature of the source is clearly shown. Also, there is a prominent shadowing effect on the neutron fluence due to the vehicle walls and the LINAC shielding. Since the remainder of the geometry is filled with air, there is a negligible attenuation of the neutrons. Instead, the flux is simply reduced in the typical $1 / r^{2}$ manner. 
Figure 52 and Figure 53 show the neutron field just inside the LINAC shielding above and below the beamline; the color scale is held constant in order to make a direct comparison to all other figures. As expected, these two neutron fields are fully symmetric about the geometric midplane. Figure 54 shows the fluence just below the LINAC shielding; however, the plotting plane is still close enough to the shielding to observe the shadow. Figure 55 and Figure 56 show the neutron fluence at two locations between LINAC shielding and the vehicle wall. The shadowing effect from the LINAC shielding is no longer visible. Figure 57 shows the results between the vehicle and the soil. The shadowing effect from the vehicle walls is no longer visible.

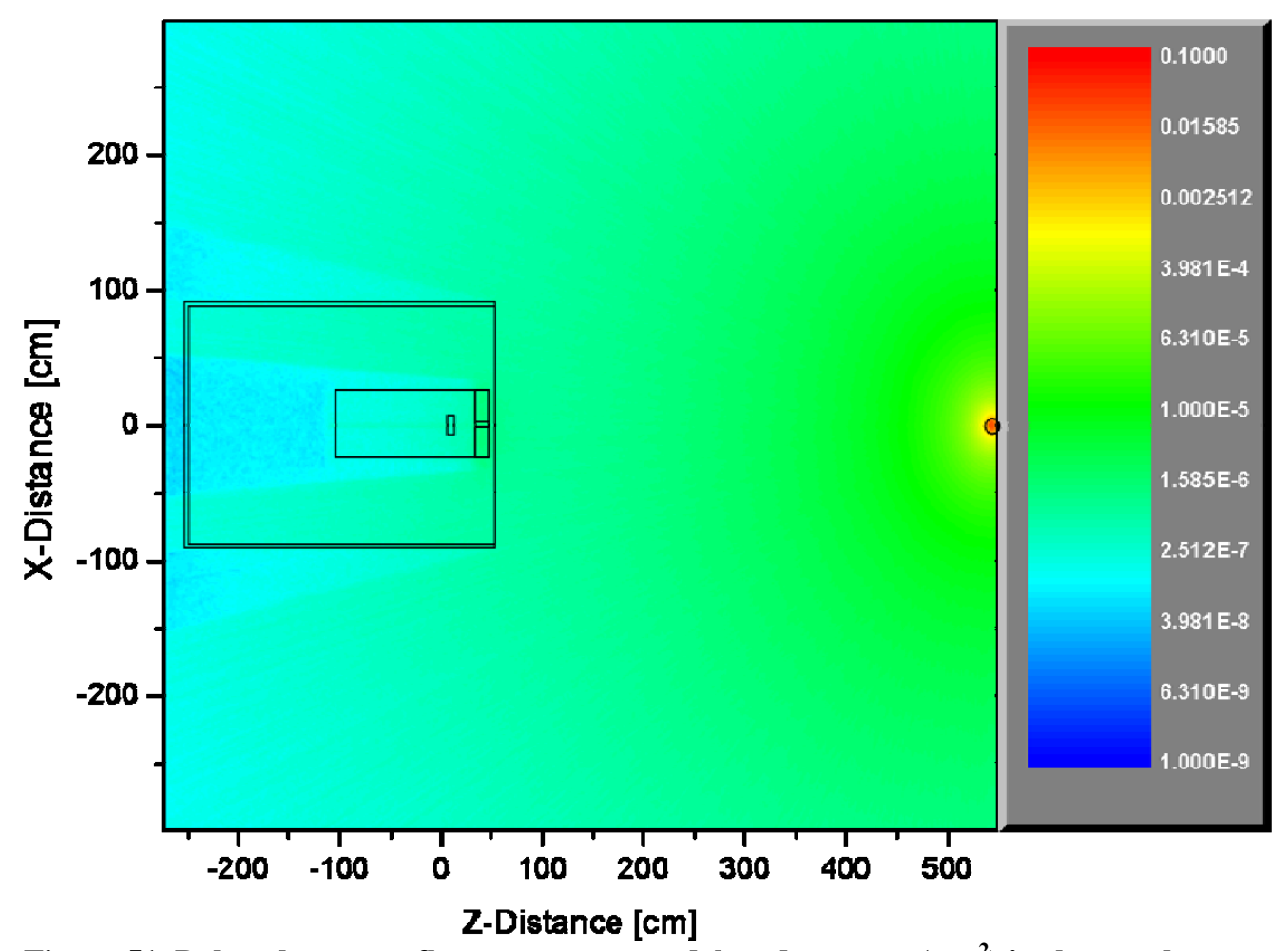

Figure 51. Delayed neutron fluence per source delayed neutron $\left(\mathrm{cm}^{-2}\right)$ in the $x-z$ plane centered about $y=0 \mathrm{~cm}$. This is the center-plane of the geometry. 


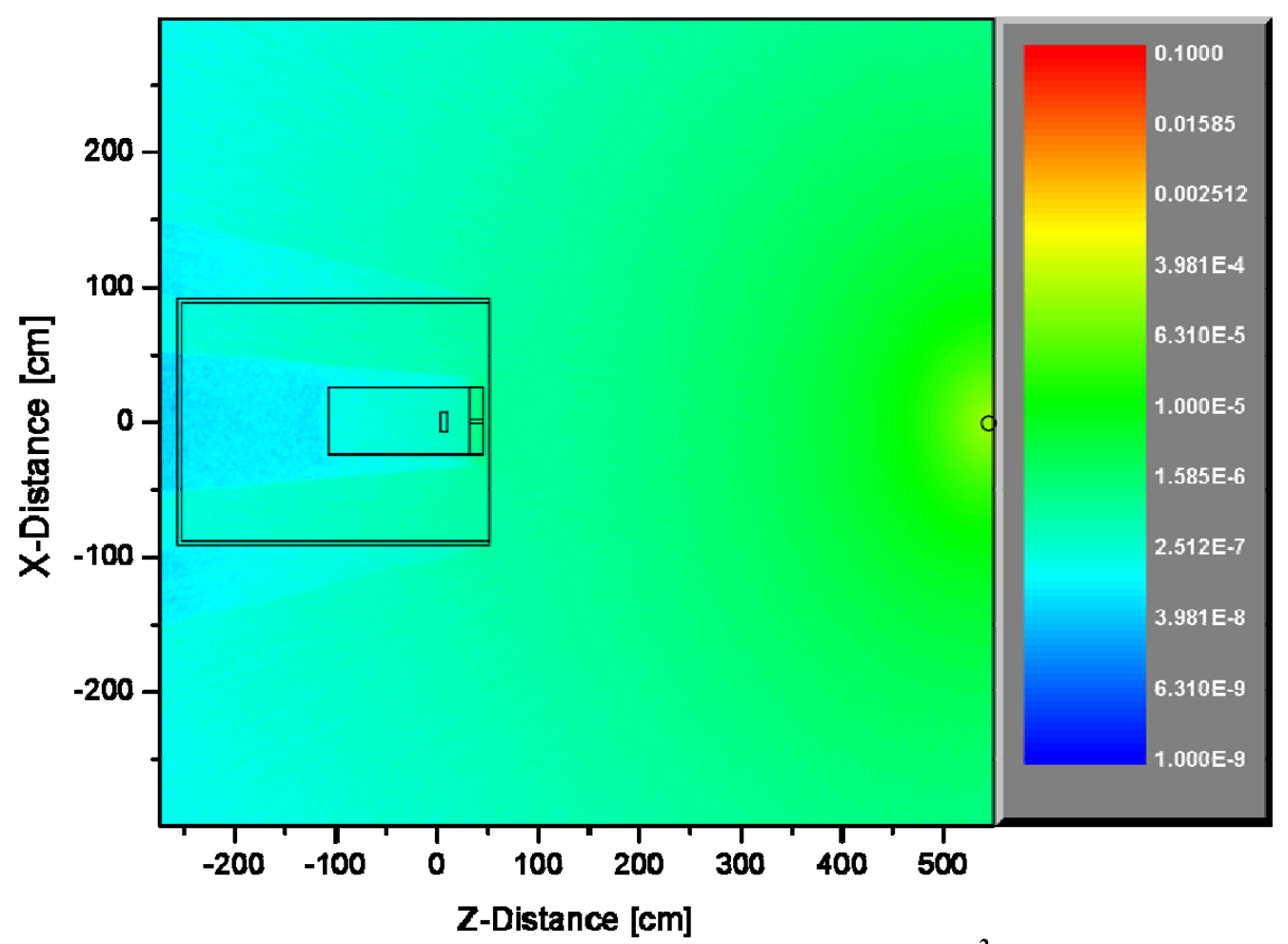

Figure 52. Delayed neutron fluence per source delayed neutron $\left(\mathrm{cm}^{-2}\right)$ in the $x$-z plane centered about $y=24 \mathrm{~cm}$.

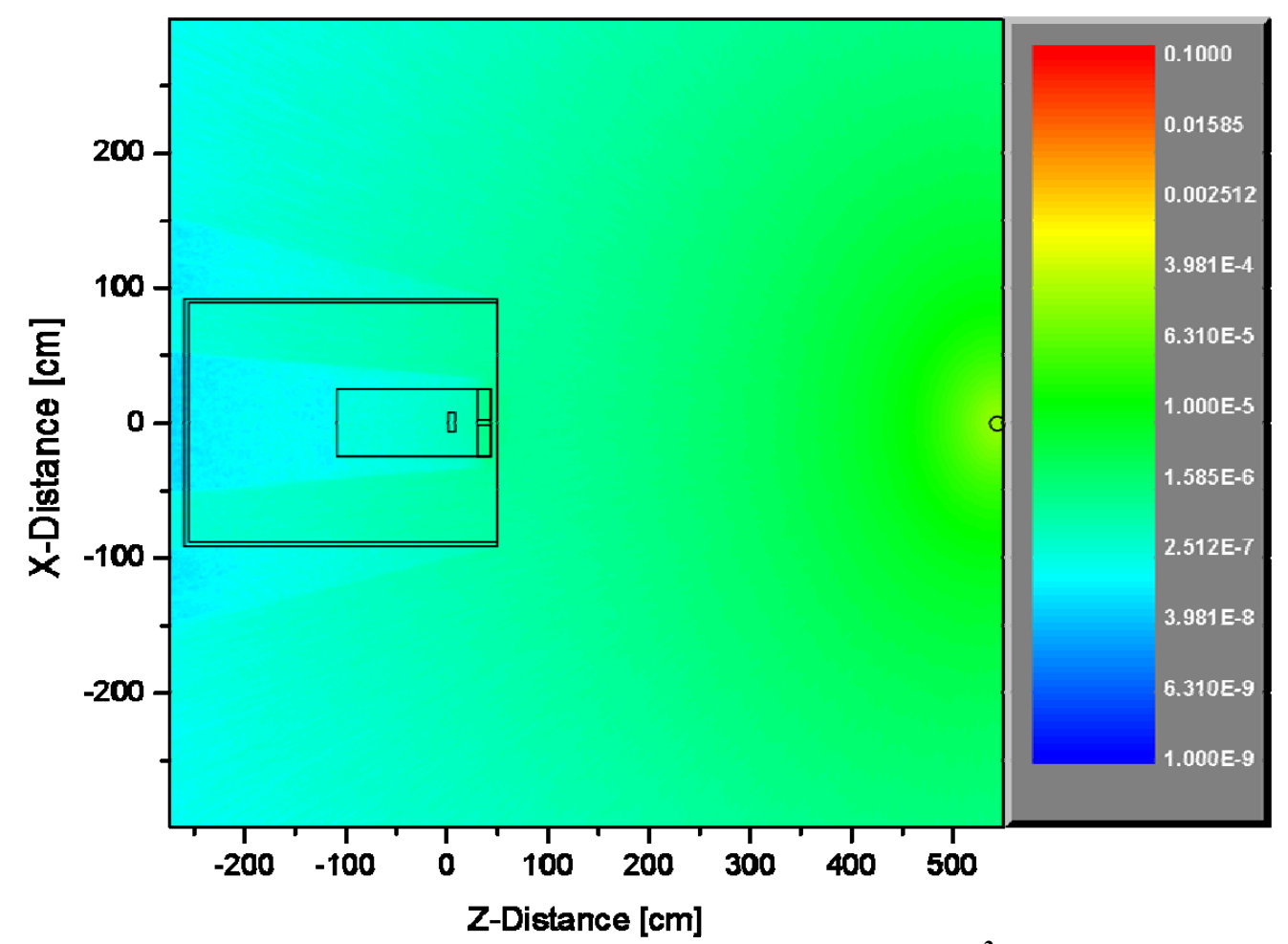

Figure 53. Delayed neutron fluence per source delayed neutron $\left(\mathrm{cm}^{-2}\right)$ in the $x-z$ plane centered about $y=-24 \mathrm{~cm}$. 


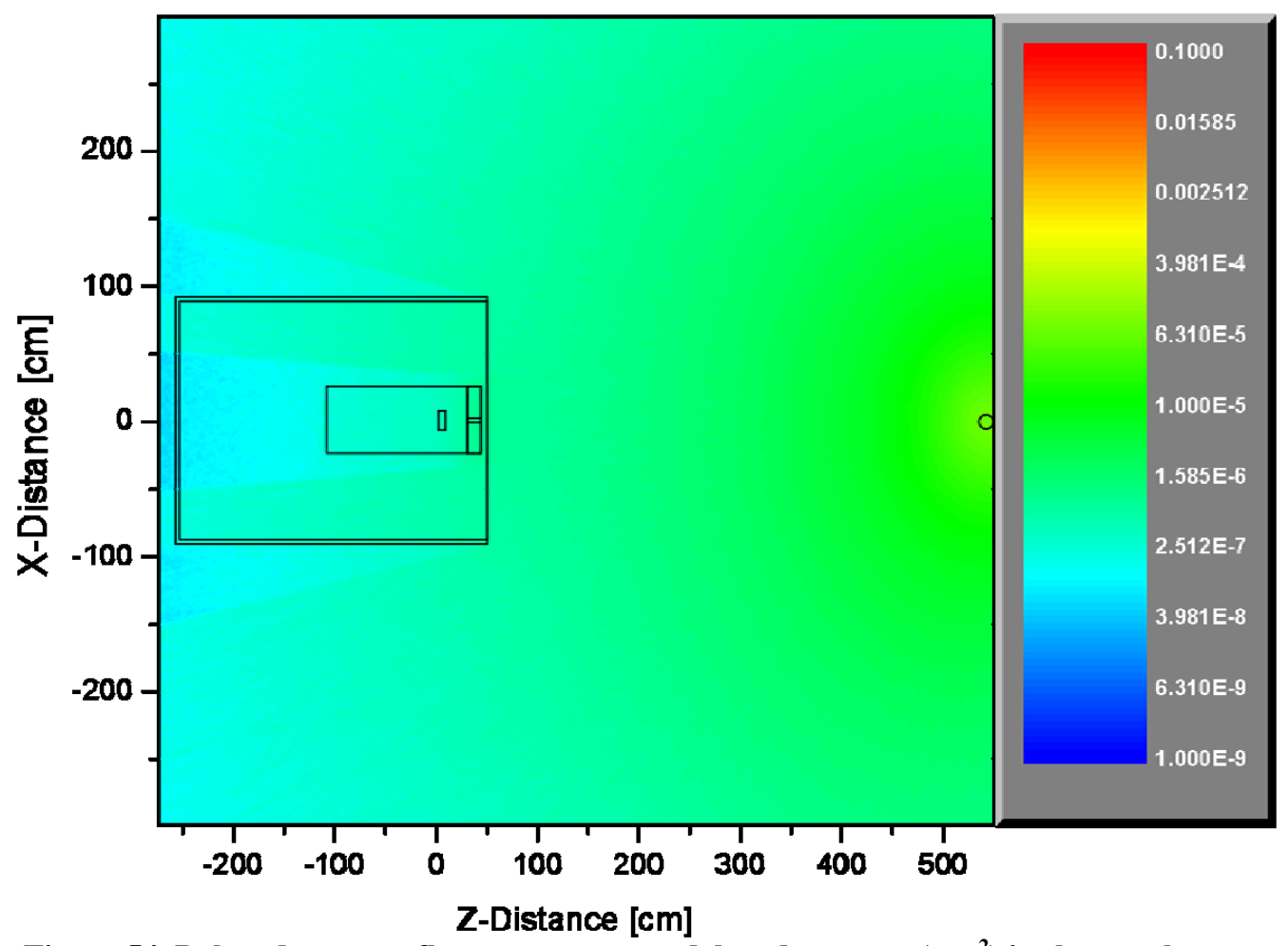

Figure 54. Delayed neutron fluence per source delayed neutron $\left(\mathrm{cm}^{-2}\right)$ in the $x-z$ plane centered about $y=-33.62 \mathrm{~cm}$.

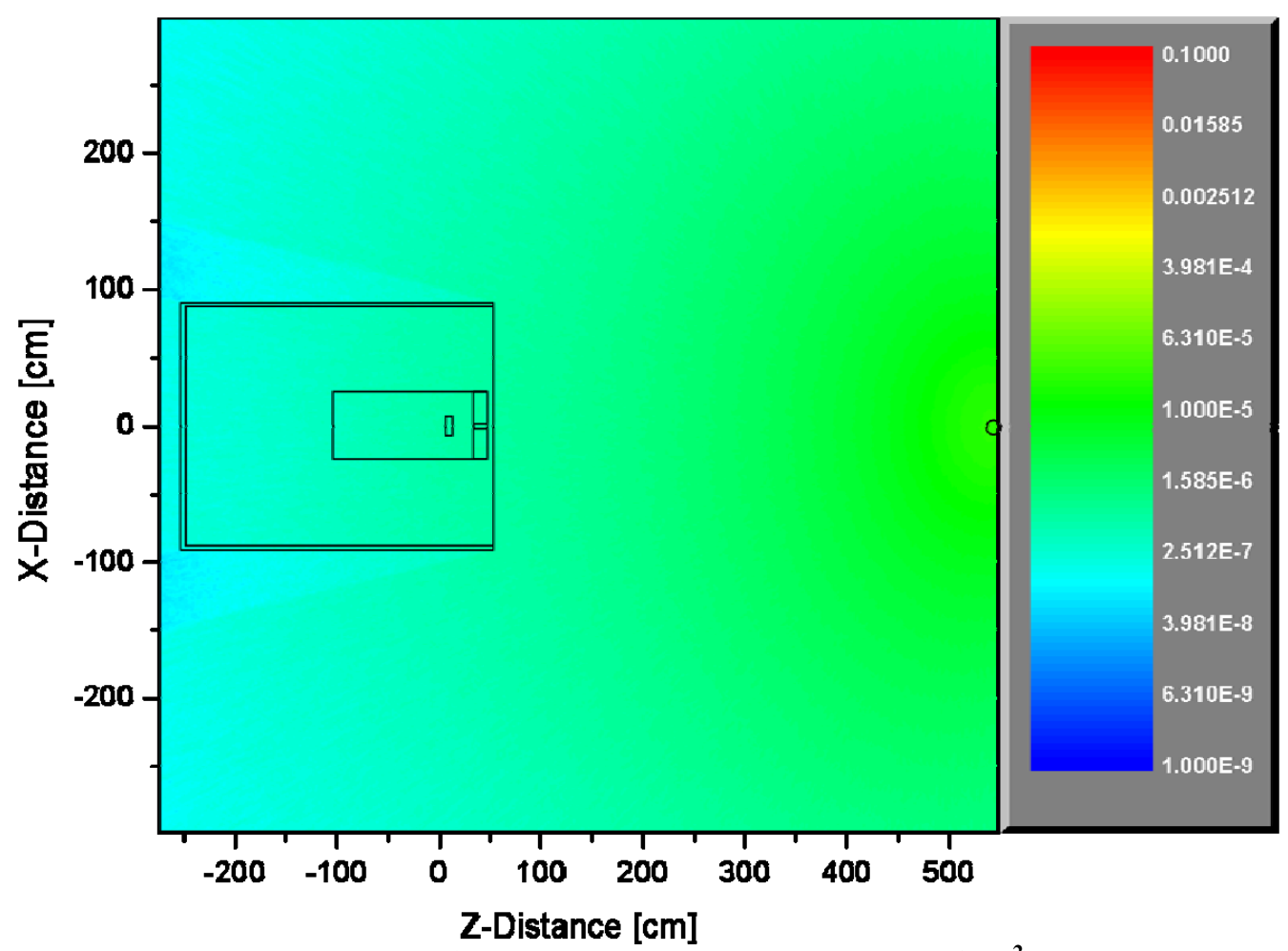

Figure 55. Delayed neutron fluence per source delayed neutron $\left(\mathrm{cm}^{-2}\right)$ in the $x-z$ plane centered about $\mathrm{y}=-62 \mathrm{~cm}$. 


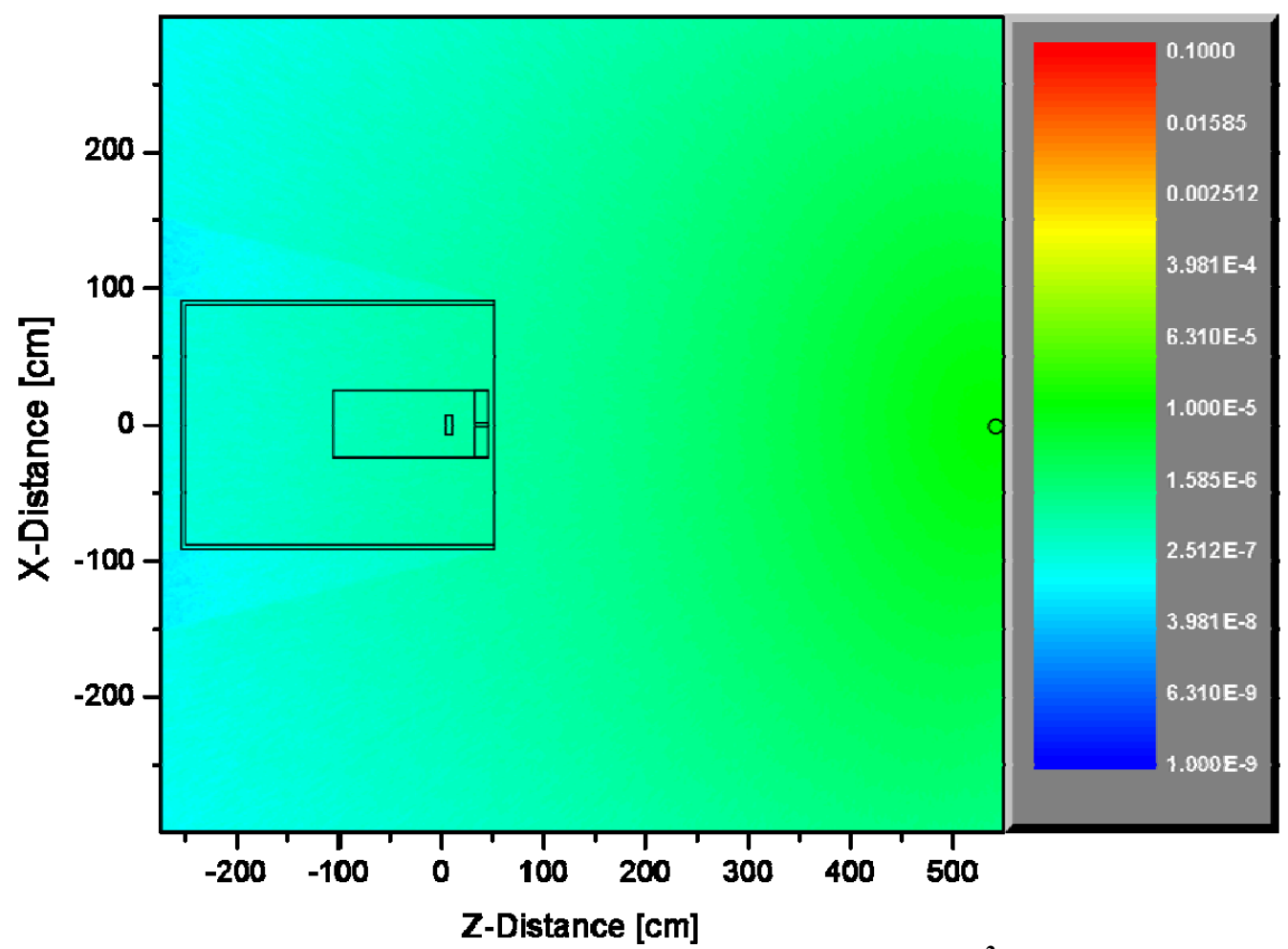

Figure 56. Delayed neutron fluence per source delayed neutron $\left(\mathrm{cm}^{-2}\right)$ in the $x-z$ plane centered about $y=-90.44 \mathrm{~cm}$.

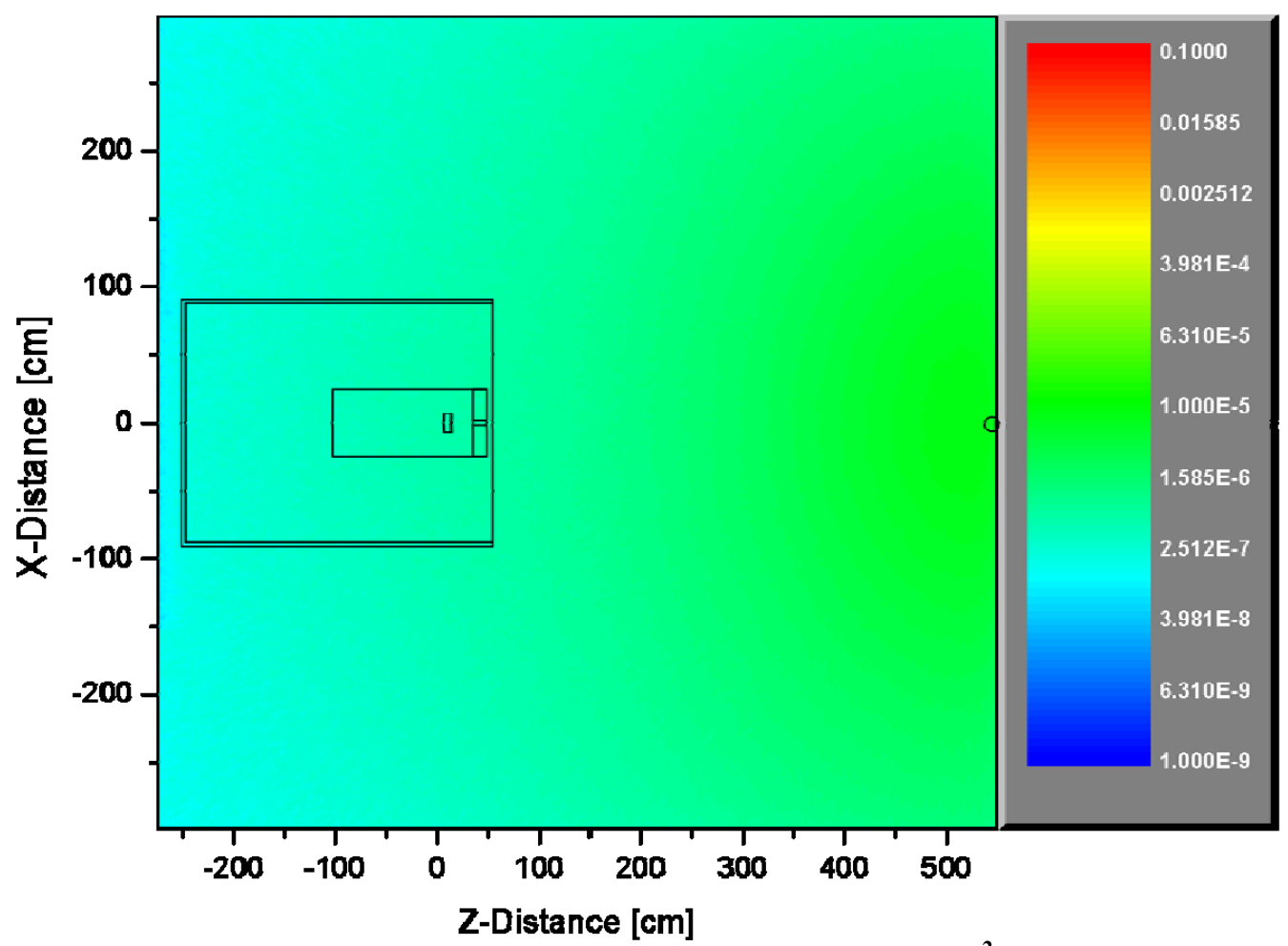

Figure 57. Delayed neutron fluence per source delayed neutron $\left(\mathrm{cm}^{-2}\right)$ in the $x$-z plane centered about $y=-149 \mathrm{~cm}$. 
These results are not directly comparable to the prompt neutron results shown in Section 4 because the units are inconsistent - the prompt results are per source electron, whereas the delayed results are per source delayed neutron. Two primary conversion factors are needed to carry out a reasonable, albeit approximate, comparison of these results:

1. The number of fissions occurring in the target per source electron

2. The number of delayed neutrons released per fission $(v \beta)$ of ${ }^{238} \mathrm{U}$

The second of these factors is readily available for neutron-induced fission [3]: $v=2.60$ and $\beta=0.0157$. As a first approximation, it will be applied here to photon-induced fission. The first factor may be estimated from the MCNPX results to be $4.5 \mathrm{e}-6$ reactions per electron. The delayed neutron fluence is multiplied by the product of these two factors to make a comparison to the prompt results. Figure 58 shows these results for the geometric mid-plane. A visual comparison reveals that the ratio between the delayed neutron field and the prompt neutron field, shown in Figure 22, is approximately $10^{-7}$.

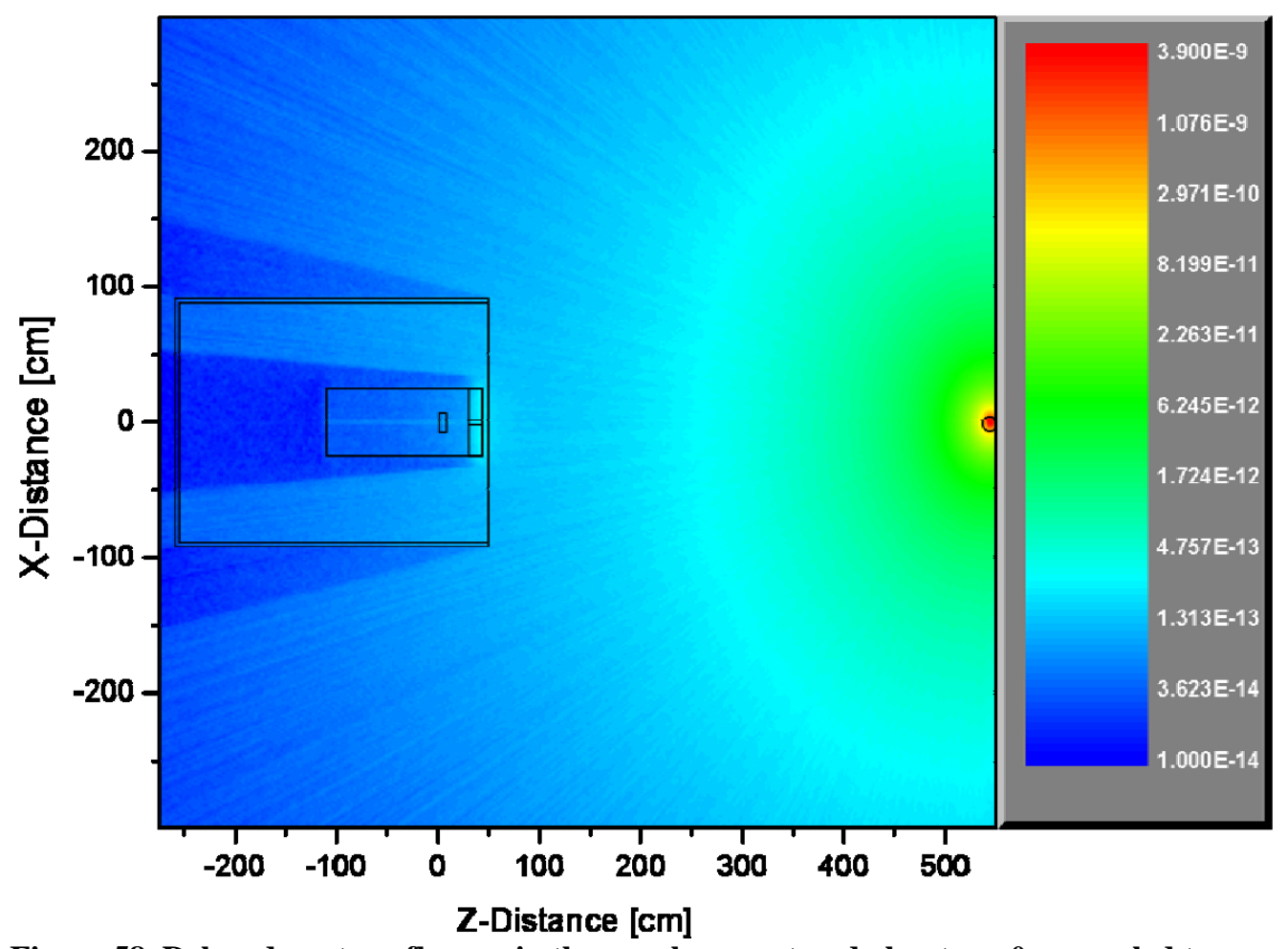

Figure 58. Delayed neutron fluence in the $x$-z plane centered about $y=0$-cm scaled to reflect production per source electron.

\section{CONCLUSIONS}

Monte Carlo models of an active interrogation system, including the accelerator structure, transport vehicle walls, and the surrounding environment (air and soil) have been simulated using specialized versions of MCNPX and MCNP-PoliMi. Both prompt and delayed emissions from 
the system, including the accelerator converter and shielding and a depleted uranium target, have been mapped in three dimensions. The time-dependence of the prompt neutrons in the system has also been characterized

Both the prompt and delayed neutrons and gamma rays from the bare target were characterized. The number of delayed neutrons emitted from the target is approximately seven orders of magnitude less than the total number of prompt neutrons emitted from the system. Therefore, in order to possibly detect the delayed neutrons, the detectors should be active only after all prompt neutrons have scattered out of the system. Preliminary results have shown this time to be greater than $5 \mu$ s after the accelerator pulse.

These models have also been analyzed to determine the most likely location of the detectors. The flux maps generated with the Monte Carlo model indicate that the detectors should be placed approximately $50 \mathrm{~cm}$ behind the exit of the accelerator, $40 \mathrm{~cm}$ away from the vehicle, and $150 \mathrm{~cm}$ above the ground. This position minimizes the number of neutrons coming from the accelerator structure, which must scatter backward and also receives the maximum flux of prompt neutrons coming from the source. The lead shielding around the accelerator minimizes the gamma ray background from the accelerator in this area.

This type of system is illustrative of a host of real-world scenarios of interest to nonproliferation and homeland security, including active interrogation at a standoff. Due to the multistep procedure of the MCNPX/MCNP-PoliMi code system, the analysis of somewhat modularmeaning that changing details such as the detector type, position, or surroundings does not require a recalculation of the source-target interactions. This feature allows for efficient parametric analysis of numerous system parameters without re-computing the constant source-target behavior. Such efficient analysis mechanisms could prove invaluable in the design and future deployment of an active interrogation detection system. In the future a complete analysis of detector performance and source classification is planned. The results will also be validated through comparison with experimental data.

\section{ACKNOWLEDGMENT}

Oak Ridge National Laboratory is managed and operated for the Department of Energy by UTBattelle, LLC, under contract DE-AC05-00OR22725. This work was supported in part by the Defense Threat Reduction Agency (DTRA) and was performed under a Work-for-Others contract with NucSafe, Inc. of Oak Ridge, Tennessee.

\section{REFERENCES}

1. X-5 Monte Carlo Team, MCNP-A General Monte Carlo N-Particle Transport Code, Version 5, vols. 1-3. Los Alamos National Laboratory. LA-UR-03-1987, LA-CP-03-0245, and LA-CP-03-0284 (2003).

2. S. A. Pozzi, E. Padovani, and M. Marseguerra, "MCNP-PoliMi: A Monte Carlo Code for Correlation Measurements," Nuclear Instruments and Methods, A513: 550-558 (2003).

3. Karl O. Ott and Robert J. Neuhold, Introductory Nuclear Reactor Dynamics. American Nuclear Society, La Grange Park, IL (1985). 
APPENDIXES 

APPENDIX A

MCNPX INPUT FILE FOR SOURCE CHARACTERIZATION 



\section{Appendix A. MCNPX Input File for Source Chracterization}

\section{Electron Source:}

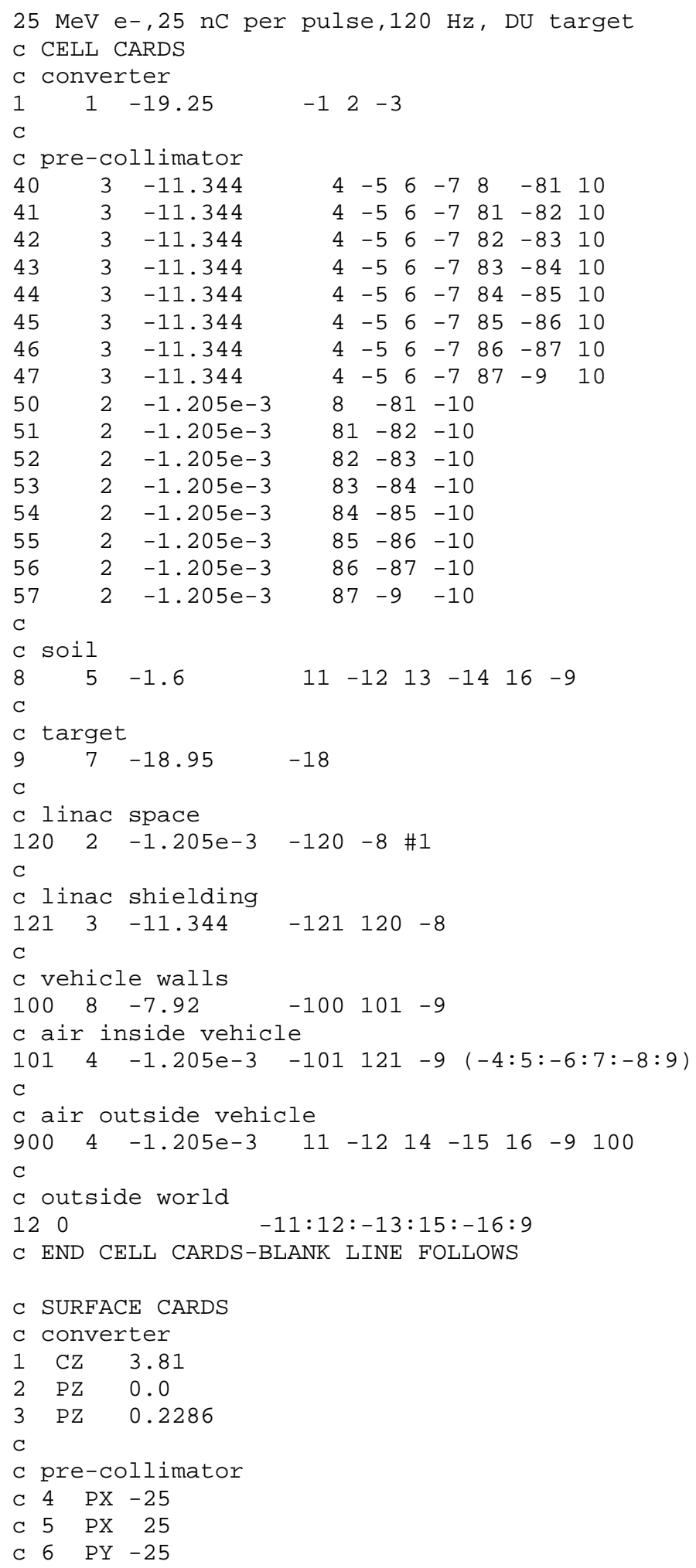




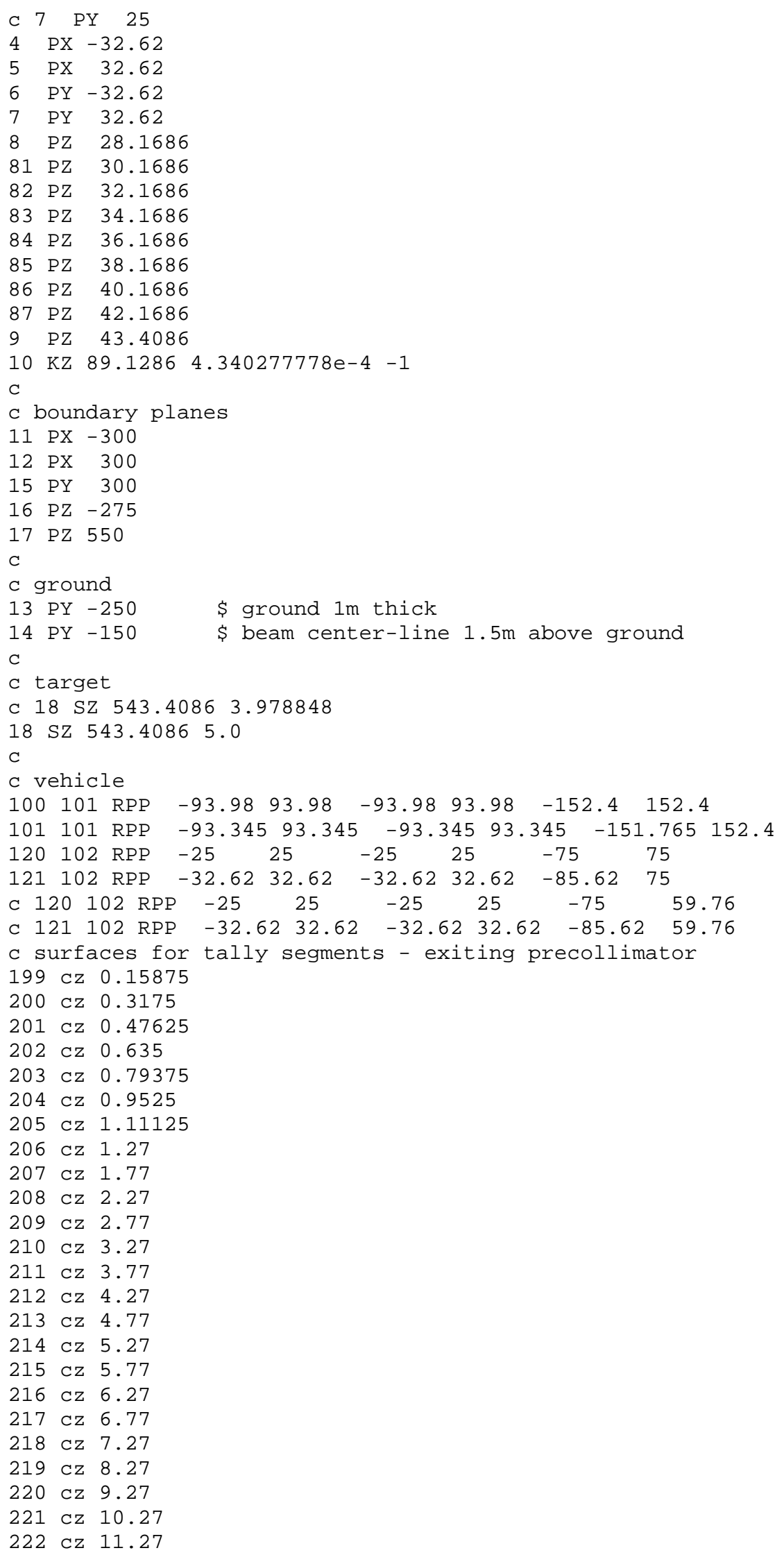




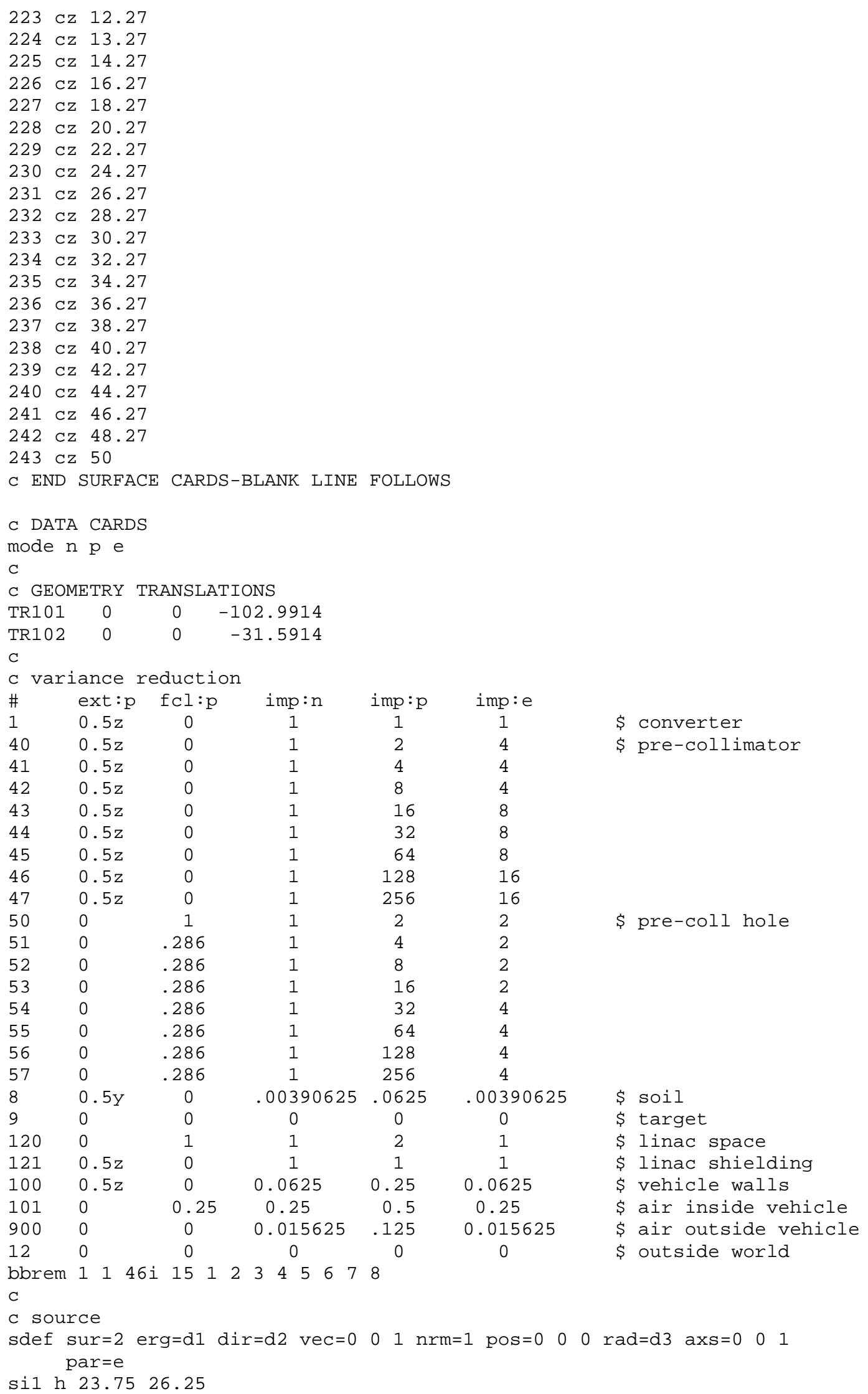


sp1 d $\odot \quad 1$

si2 h $-1 \quad 0.9998476951$

sp2 d $00 \%$

si3 a $\odot \quad 0.25$

sp3 $\odot \quad 0.25$

$\mathrm{C}$

c tallies

c neutron

f1:n 9

fm1 $1.872453 \mathrm{e} 13$

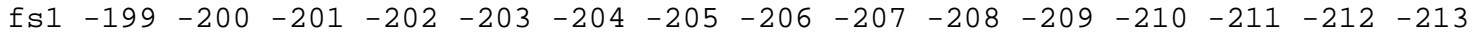

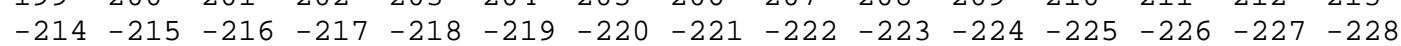

$\begin{array}{lllllllllllllll}-229 & -230 & -231 & -232 & -233 & -234 & -235 & -236 & -237 & -238 & -239 & -240 & -241 & -242 & -243\end{array}$

$\mathrm{t}$

e1 .2 7 i 1 11i 4 4.25 $102 i 30$

c photon

f11:p 9

fm11 $1.872453 e 13$

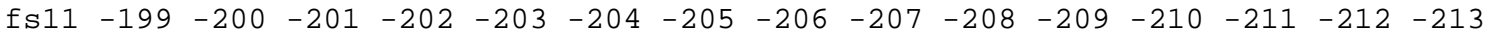

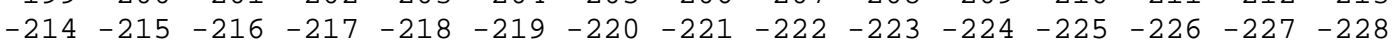

$\begin{array}{lllllllllllllll}-229 & -230 & -231 & -232 & -233 & -234 & -235 & -236 & -237 & -238 & -239 & -240 & -241 & -242 & -243\end{array}$

$\mathrm{t}$

c electron

f21:e 9

fm21 $1.872453 \mathrm{e} 13$

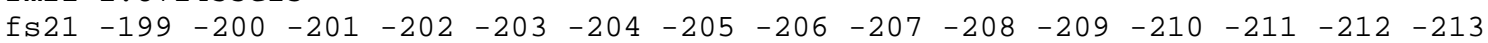

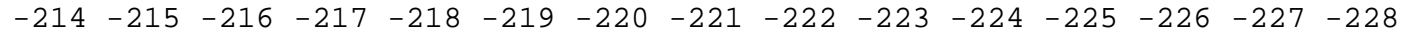

$\begin{array}{lllllllllllllllll}-229 & -230 & -231 & -232 & -233 & -234 & -235 & -236 & -237 & -238 & -239 & -240 & -241 & -242 & -243\end{array}$ $\mathrm{t}$

c all tally parameters

e० $4.25102 i 30$

c * ${ }^{*} 0 \begin{array}{lllllllllllllllllllllllllllll}90 & 80 & 70 & 60 & 50 & 40 & 35 & 30 & 25 & 20 & 15 & 10 & 7.5 & 5 & 2.5 & 2.4 & 2.3 & 2.2 & 2.1 & 2 & 1.9 & 1.8\end{array}$

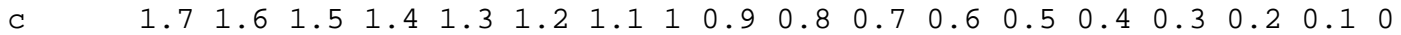

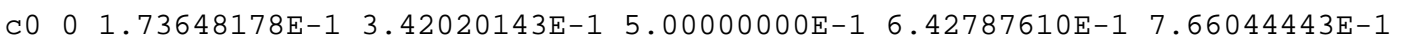
8.19152044E-1 8.66025404E-1 9.06307787E-1 9.39692621E-1 9.65925826E-1 $9.84807753 \mathrm{E}-1 \quad 9.85109326 \mathrm{E}-1 \quad 9.85407898 \mathrm{E}-1 \quad 9.85703469 \mathrm{E}-1$ $9.85996037 \mathrm{E}-1 \quad 9.86285602 \mathrm{E}-1 \quad 9.86572162 \mathrm{E}-1 \quad 9.86855716 \mathrm{E}-1 \quad 9.87136265 \mathrm{E}-1$ 9.87413807E-1 9.87688341E-1 9.87959866E-1 9.88228381E-1 9.88493887E-1 $9.88756381 \mathrm{E}-1$ 9.89015863E-1 9.89272333E-1 $9.89525789 \mathrm{E}-1$ 9.89776231E-1 9.90023658E-1 9.90268069E-1 9.90509463E-1 9.90747840E-1 9.90983200E-1 9.91215540E-1 9.91444861E-1 9.91671162E-1 9.91894443E-1 9.92114701E-1 9.92331938E-1 9.92546152E-1 9.92757342E-1 9.92965508E-1 9.93170650E-1 9.93372766E-1 9.93571856E-1 9.93767919E-1 $9.93960955 \mathrm{E}-1 \quad 9.94150964 \mathrm{E}-1$ 9.94337944E-1 9.94521895E-1 9.94702817E-1 9.94880709E-1 9.95055570E-1 9.95227400E-1 9.95396198E-1 9.95561965E-1 9.95724698E-1 9.95884399E-1 9.96041065E-1 9.96194698E-1 9.96345296E-1 9.96492859E-1 9.96637387E-1 9.96778878E-1 9.96917334E-1 9.97052752E-1 9.97185134E-1 9.97314477E-1 $\begin{array}{lllll}9.97440783 \mathrm{E}-1 & 9.97564050 \mathrm{E}-1 & 9.97684279 \mathrm{E}-1 & 9.97801468 \mathrm{E}-1 & 9.97915618 \mathrm{E}-1\end{array}$ 9.98026728E-1 9.98134798E-1 9.98239828E-1 9.98341817E-1 9.98440764E-1 $9.98536670 \mathrm{E}-1 \quad 9.98629535 \mathrm{E}-1 \quad 9.98719357 \mathrm{E}-1 \quad 9.98806137 \mathrm{E}-1 \quad 9.98889875 \mathrm{E}-1$ 9.98970570E-1 9.99048222E-1 9.99122830E-1 9.99194395E-1 9.99262916E-1 9.99328394E-1 9.99390827E-1 9.99450216E-1 9.99506560E-1 9.99559860E-1 9.99610115E-1 9.99657325E-1 9.99701490E-1 9.99742609E-1 9.99780683E-1 9.99815712E-1 9.99847695E-1 9.99876632E-1 9.99902524E-1 9.99925370E-1 9.99945169E-1 9.99961923E-1 9.99975631E-1 9.99986292E-1 9.99993908E-1 9. $99998477 \mathrm{E}-11 \mathrm{t}$

C

c materials

m1 nlib=66c plib=04p elib=03e pnlib=26u gas=0 estep=4 $\$$ tungsten

$\begin{array}{llllllll}74182 & 0.2642 & 74183 & 0.1428 & 74184.24 u & 0.307 & 74186 & 0.286\end{array}$

m2 nlib=66c plib=๑4p elib=๑3e pnlib=25u gas=1 estep=210 $\$$ air between $W \& P b$

$45 \%$ humidity

1001 1.169196E-02 1002.24U 1.344730E-06 
$70147.681679 \mathrm{E}-0170152.837299 \mathrm{E}-03$

$8016.24 \mathrm{U} \quad 2.121736 \mathrm{E}-0180175.168377 \mathrm{E}-04$

$18000.59 \mathrm{C} 4.611104 \mathrm{E}-03$

c $m \times 2: p \circ 5 j 18040$

mpn2 $\odot 1002701470158016801718040$

m3 nlib=66c plib=04p elib=03e pnlib=24u gas=0 estep=4 $\$$ lead

$82206 \quad 0.25582207 \quad 0.22182208 \quad 0.524$

m4 nlib=66c plib=04p elib=03e pnlib=25u gas=1 estep=12 \$ air everywhere else $45 \%$ humidity

1001 1.169196E-02 1002.24U 1.344730E-๑6

$70147.681679 \mathrm{E}-0170152.837299 \mathrm{E}-03$

$8016.24 \mathrm{U} 2.121736 \mathrm{E}-0180175.168377 \mathrm{E}-04$

$18000.59 \mathrm{c} 4.611104 \mathrm{E}-03$

c $m \times 4: p \circ 5 j 18040$

mpn4 ๑ 1002701470158016801718040

m5 nlib=66c plib=04p elib=03e pnlib=25u gas=0 estep=3 \$ LANL soil + $20 \%$ water 1001 4.832564E-02 1002.24U 5.558087E-06

$8016.24 \mathrm{U} \quad 6.950058 \mathrm{E}-0180171.692978 \mathrm{E}-03$

$110233.155206 \mathrm{E}-03$

$130273.045777 \mathrm{E}-02$

14028 1.934418E-01 $140299.822504 \mathrm{E}-03$ 1403९ $6.475067 \mathrm{E}-\odot 3$

$190004.523070 \mathrm{E}-03$

26054 4.146826E-๑4 $26056 \quad 6.509629 \mathrm{E}-03 \quad 26057 \quad 1.503357 \mathrm{E}-\odot 4$

$260582.000693 E-05$

c $m \times 5: p \circ 8 j 190394 j$

mpn5 ๑ $1002801680171102313027 \quad 14028140291403019039$ 26054260562605726058

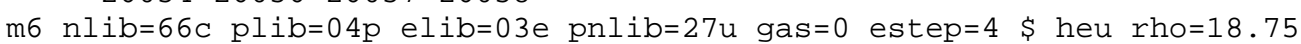
$922350.9892238 \quad 0.02$

m7 nlib=66c plib=04p elib=03e pnlib=27u gas=0 estep=4 $\$$ du rho=18.95 922381

C stainless steel

M8 NLIB $=66 \mathrm{c}$ PLIB $=04 p$ elib=03e pnlib=25u gas= 0 estep=5

$260543.470 \mathrm{e}-3$

$260565.447 \mathrm{e}-2$

$260571.258 \mathrm{e}-3$

$240507.573 e-4$

$240521.450 \mathrm{e}-2$

$240531.656 \mathrm{e}-3$

$24054 \quad 4.122 e-4$

$280585.288 \mathrm{e}-3$

$280602.024 \mathrm{e}-3$

$280647.148 \mathrm{e}-4$

$250551.740 \mathrm{e}-3$

C

TOTNU

$c$ energy and thermal treatment

phys: $n 30$

phys:p $302 j-10$

phys:e 30

c cut off

cut:n j 0.1

cut:p j 4.0

cut:e j 4.0

nps 5 e3

c ctme 7200

c peripheral

prdmp j 1 e8 j 21 e8

print

c dbcn 7j $14 j \mathrm{j}$

rand gen $=2$ stride $=611671$

$c$ end of file 

APPENDIX B

MCNPX INPUT FILE FOR LINAC BEAM CHARACTERIZATION 



\section{Appendix B. MCNPX Input Files for LINAC Beam Characterization}

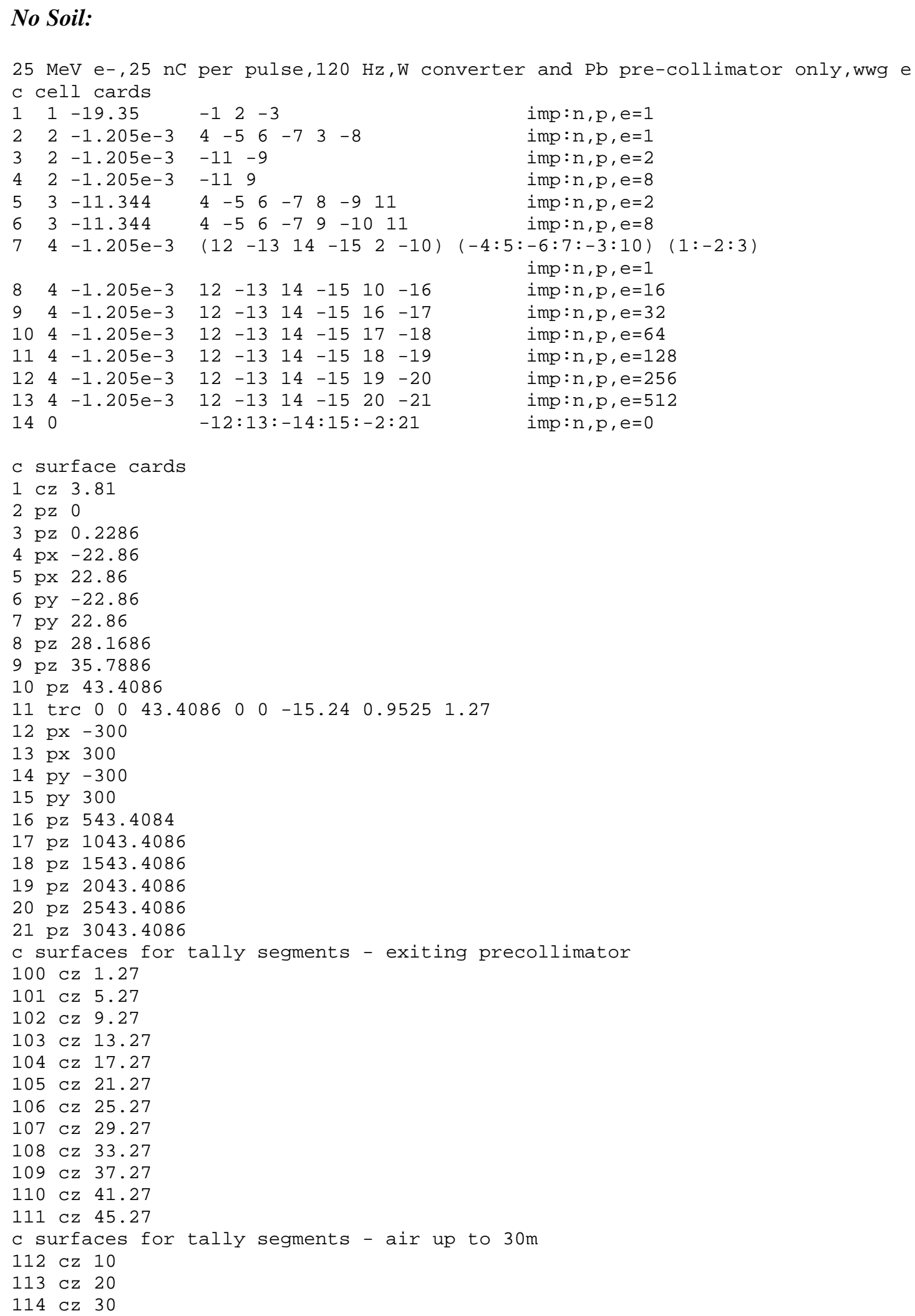




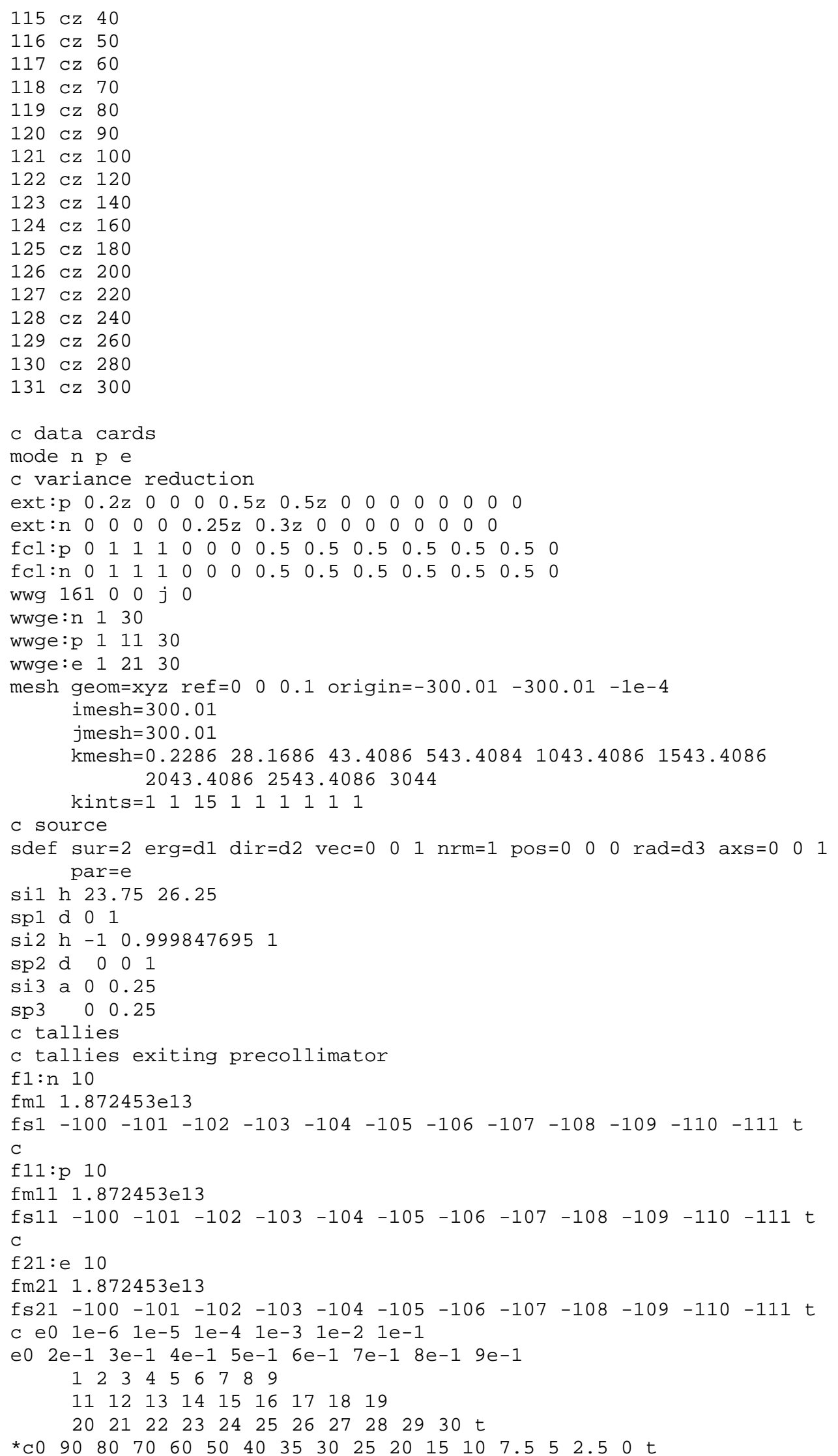




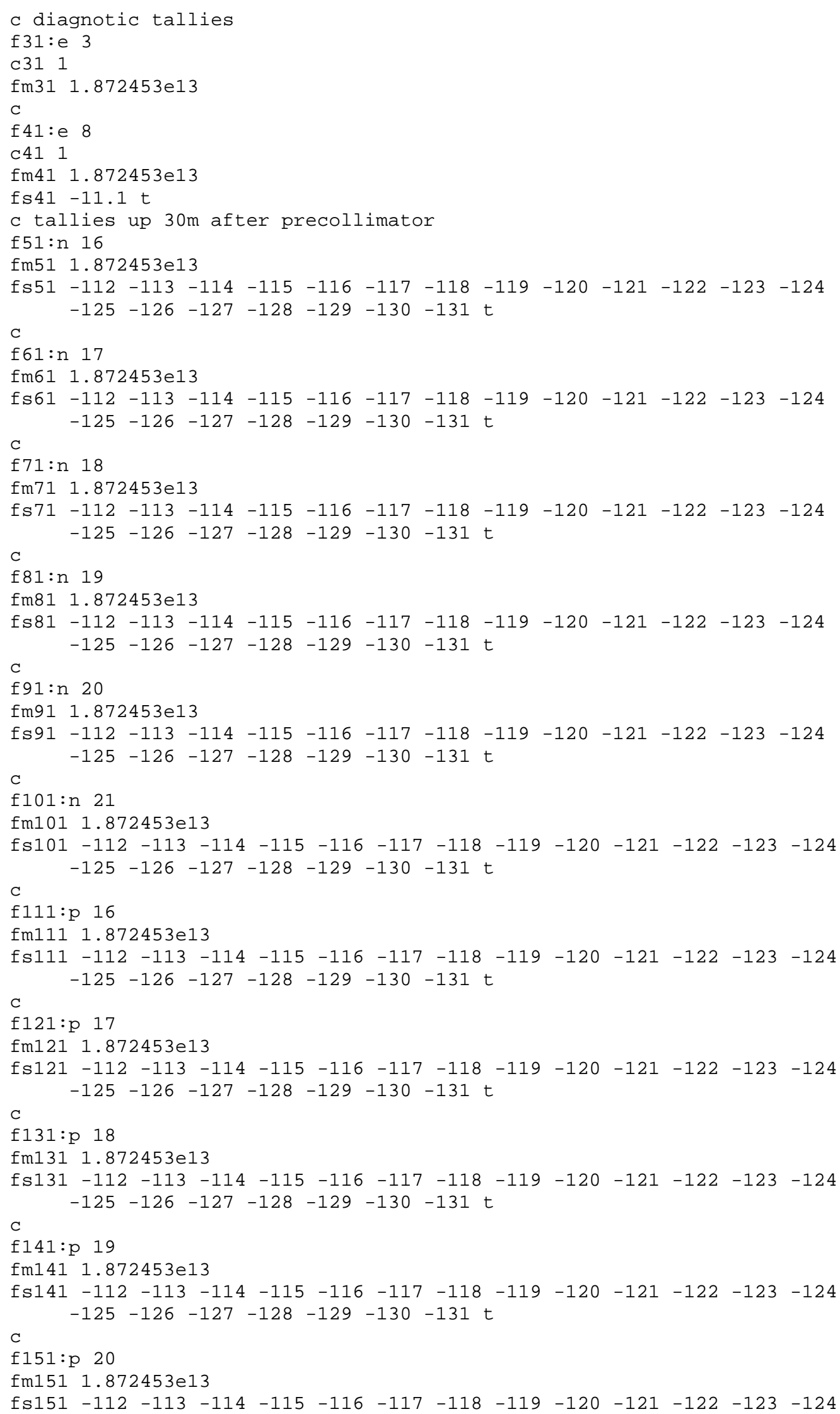




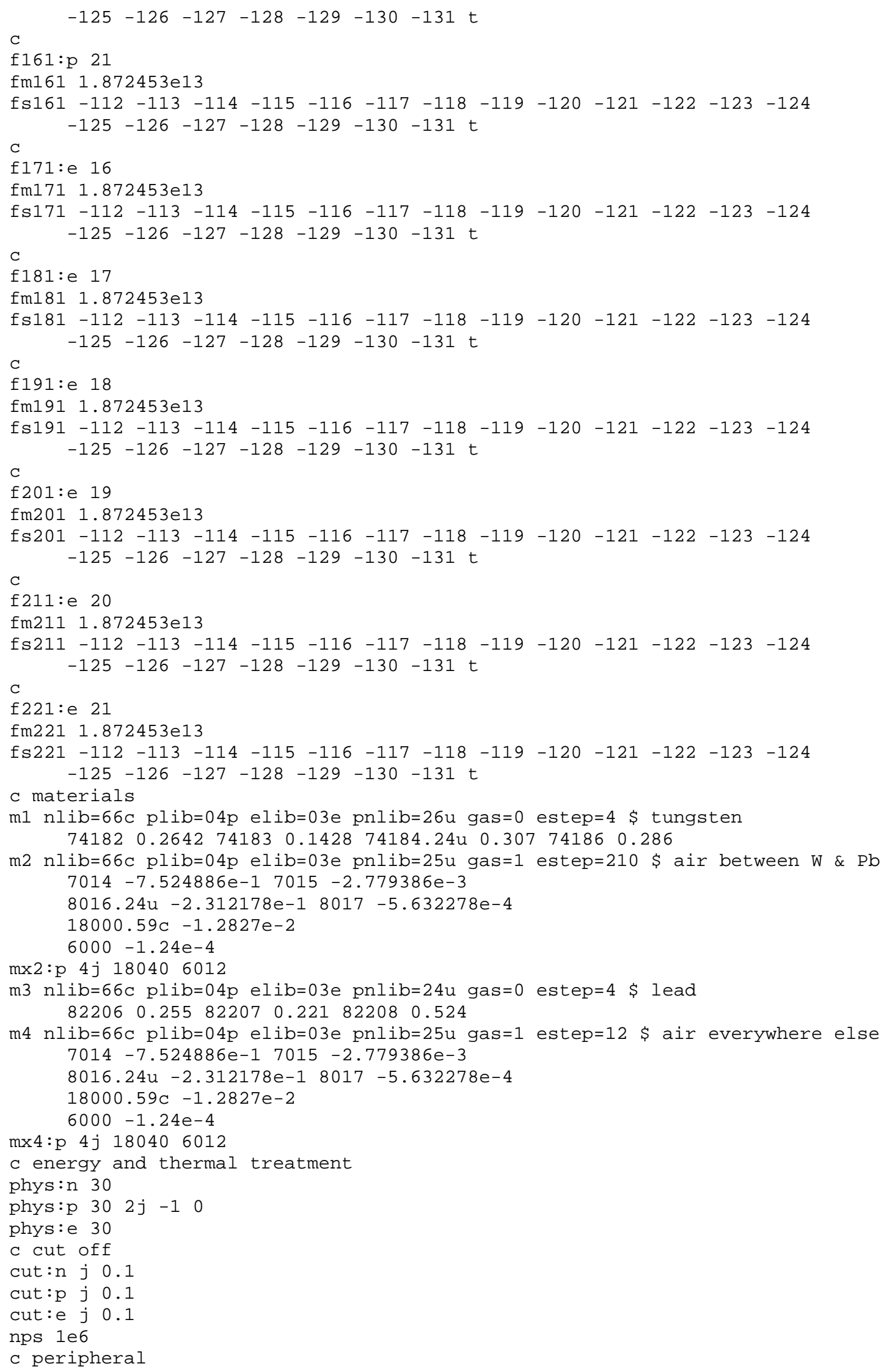


prdmp $3 j 2$

print

dbcn $7 j$ j $4 j 458751$

$c$ end of file

\section{With Soil:}

$25 \mathrm{MeV}$ e-,25 nc per pulse, $120 \mathrm{~Hz}, \mathrm{~W}$ converter Pb pre-collimator \& soil c cell cards

$1 \quad 1-19.35$

$\begin{array}{lll}-1 & 2 & -3\end{array}$

$2-1.205 e-3 \quad-11-9$

$2-1.205 e-3 \quad-119$

$\begin{array}{lllllllll}3 & -11.344 & 4 & -5 & 6 & -7 & 8 & -9 & 11\end{array}$

$\begin{array}{lllllllll}3 & -11.344 & 4 & -5 & 6 & -7 & 9 & -10 & 11\end{array}$

$7 \quad 4-1.205 e-3$

$\left(\begin{array}{llllll}12 & -13 & 14 & -15 & 2 & -10\end{array}\right)$

$84-1.205 e-3$

$\begin{array}{llllll}12 & -13 & 14 & -15 & 10 & -16\end{array}$

$\begin{array}{lllllllll}9 & 4 & -1.205 e-3 & 12 & -13 & 14 & -15 & 16 & -17\end{array}$

$\begin{array}{lllllllll}10 & 4 & -1.205 e-3 & 12 & -13 & 14 & -15 & 17 & -18\end{array}$

$\begin{array}{lllllllll}11 & 4 & -1.205 \mathrm{e}-3 & 12 & -13 & 14 & -15 & 18 & -19\end{array}$

$\begin{array}{lllllllll}12 & 4 & -1.205 e-3 & 12 & -13 & 14 & -15 & 19 & -20\end{array}$

$\begin{array}{lllllllll}13 & 4 & -1.205 e-3 & 12 & -13 & 14 & -15 & 20 & -21\end{array}$

$145-1.6$

$\begin{array}{llllll}12 & -13 & -14 & 22 & 2 & -10\end{array}$

$\begin{array}{llllll}12 & -13 & -14 & 22 & 10 & -16\end{array}$

$\begin{array}{llllll}12 & -13 & -14 & 22 & 16 & -17\end{array}$

$\begin{array}{llllll}12 & -13 & -14 & 22 & 17 & -18\end{array}$

$\begin{array}{llllll}12 & -13 & -14 & 22 & 18 & -19\end{array}$

$\begin{array}{llllll}12 & -13 & -14 & 22 & 19 & -20\end{array}$

$\begin{array}{llllll}12 & -13 & -14 & 22 & 20 & -21\end{array}$

$-12: 13:-22: 15:-2: 21$

imp: $n, p, e=1$

imp: $n, p, e=4$

imp: $n, p, e=16$

imp: $n, p, e=64$

imp: $n, p, e=16$

imp: $n, p, e=64$

$\begin{array}{lll}16 & 5 & -1.6\end{array}$

$\begin{array}{lll}17 & 5 & -1.6\end{array}$

$\begin{array}{lll}18 & 5 & -1.6\end{array}$

$\begin{array}{lll}19 & 5 & -1.6\end{array}$

$\begin{array}{lll}20 & 5 & -1.6\end{array}$

210

( - 4:5:

$-6: 7:-3: 10)$

$(1:-2: 3)$

imp: $n, p, e=128$

imp: $n, p, e=128$

imp: $n, p, e=512$

imp: $n, p, e=512$

imp: $n, p, e=1024$

imp: $n, p, e=1024$

imp: $n, p, e=0$

imp: $n, p, e=128$

imp: $n, p, e=128$

imp: $n, p, e=512$

imp: $n, p, e=512$

imp: $n, p, e=1024$

imp: $n, p, e=1024$

c surface cards

$1 \mathrm{cz} 3.81$

$2 \mathrm{pz} \odot$

$3 \mathrm{pz} \quad 0.2286$

$4 \mathrm{px}-22.86$

5 px 22.86

6 py -22.86

7 py 22.86

8 pz 28.1686

$9 \mathrm{pz} 35.7886$

10 pz 43.4086

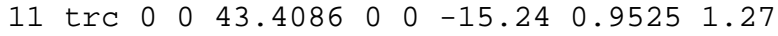

$12 \mathrm{px}-300$

$13 \mathrm{px} 300$

14 py -150

15 py 300

16 pz 543.4086

$17 \mathrm{pz} 1043.4086$

18 pz 1543.4086

$19 \mathrm{pz} 2043.4086$

$20 \mathrm{pz} 2543.4086$

21 pz 3043.4086

22 py $-250 \quad \$$ ground $1 \mathrm{~m}$ thick

c data cards

mode $\mathrm{n} p \mathrm{e}$

$c$ variance reduction

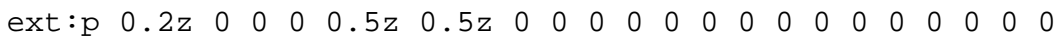

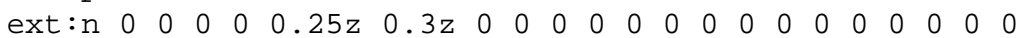

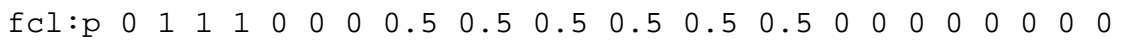


fcl:n $\odot \begin{array}{cccccccccccccccccccccccc} & 1 & 1 & \odot & \odot & \odot & 0.5 & 0.5 & \odot .5 & \odot .5 & \odot .5 & \odot .5 & \odot & \odot & \odot & \odot & \odot & \odot & \odot & \odot\end{array}$

c wwe:n 130

c wwe:p 41130

c wwe:e 42130

c wwp:n 2 j 1000 ○ -10

c wwp:p 2 j 1000 ○

c wwp:e 2 j 1000 ○ -10

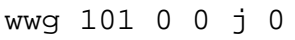

Wwge: $n 130$

Wwge: $p \quad 4 \quad 1130$

wwge:e 42130

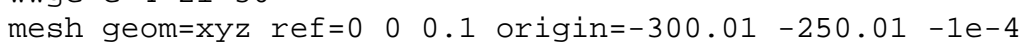

imesh $=-22.8622 .86300 .01$

jmesh $=-170 \quad-165 \quad-150 \quad-22.86 \quad 22.86 \quad 300.01$

jints $=8 \quad 1 \quad 5 \quad 1111$

kmesh $=0.2286 \quad 28.1686 \quad 43.4086 \quad 543.4086 \quad 1043.4086 \quad 1543.4086$ 2043.40862543 .40863044

kints=1 1151511111111

bbrem $11146 i 10122345$

c source

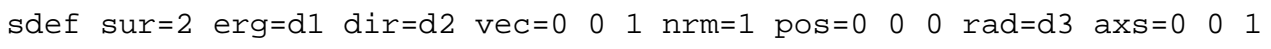
par=e

si1 h 23.7526 .25

sp1 d $\odot$ i

si2 h $-1 \quad 0.9998476951$

sp2 d $00 \%$

si3 a $\odot \quad 0.25$

sp3 $\odot \odot .25$

c tallies

f101: p 21

fm101 $1.872453 \mathrm{e} 13$

fs $14 \mathrm{t}$

tf101 $3 j 1$

e० 2e-1 3e-1 4e-1 5e-1 6e-1 7e-1 8e-1 9e-1 $1115 i 30$

$\begin{array}{llllllllllllllllll}{ }^{*} \mathrm{c} \odot & 90 & 80 & 70 & 60 & 50 & 40 & 35 & 30 & 25 & 20 & 15 & 10 & 7.5 & 5 & 2.5 & 0 & \mathrm{t}\end{array}$

c tallies up $30 \mathrm{~m}$ after precollimator

tmesh

rmesh1:p flux

cora1 $-3009 i \quad-100 \quad 19 i \quad 100 \quad 9 i 300$

corb1 -150 4i -100 19i 100 9i 300

corc1 543.4086 543.408601

rmesh11:p flux

cora11 -300 9i -100 19i 100 9i 300

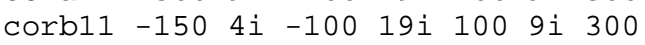

corc11 1043.4086 1043.408601

rmesh21:p flux

cora21 -300 9i -100 19i 100 9i 300

corb21 -150 4i -100 19i 100 9i 300

corc21 1543.4086 1543.408601

rmesh31:p flux

cora31 -300 9i -100 19i 100 9i 300

corb31 $-150 \quad 4 i \quad-100 \quad 19 i \quad 100 \quad 9 i \quad 300$

corc31 2043.4086 2043.408601

rmesh41:p flux

cora41 -300 9i -100 19i 100 9i 300

corb41 $-150 \quad 4 i-100 \quad 19 i \quad 100$ 9i 300

corc41 2543.4086 2543.408601

rmesh51:p flux

cora51 -300 9i -100 19i 100 9i 300

$\begin{array}{llllllllll}\text { corb51 } & -150 & 4 i & -100 & 19 i & 100 & 9 i & 300\end{array}$

corc51 3043.408599 3043.4086

endmd 
fm1 $1.872453 \mathrm{e} 13$

fm11 $1.872453 \mathrm{e} 13$

fm21 1.872453e13

fm31 $1.872453 \mathrm{e} 13$

fm41 $1.872453 \mathrm{e} 13$

fm51 1.872453e13

c materials

m1 nlib=66c plib=04p elib=03e pnlib=26u gas=0 estep=4 $\$$ tungsten

$\begin{array}{lllllll}74182 & 0.264274183 & 0.1428 & 74184.24 u & 0.307 & 74186 & 0.286\end{array}$

m2 nlib=66c $p l i b=04 p$ elib=03e pnlib=25u gas=1 estep=210 $\$$ air between $W$ \&b $7014-7.524886 \mathrm{e}-17015-2.779386 \mathrm{e}-3$

$8016.24 \mathrm{u}-2.312178 \mathrm{e}-18017-5.632278 \mathrm{e}-4$

$18000.59 \mathrm{c}-1.2827 \mathrm{e}-2$

$6000-1.24 \mathrm{e}-4$

$m \times 2: p \quad 4 j 180406012$

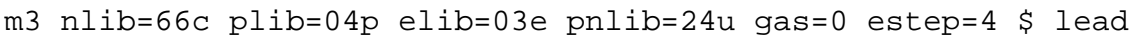
$\begin{array}{lllll}82206 & 0.25582207 & 0.22182208 & 0.524\end{array}$

m4 nlib=66c plib=04p elib=03e pnlib=25u gas=1 estep=12 $\$$ air everywhere else $7014-7.524886 e-17015-2.779386 e-3$

$8016.24 \mathrm{u}-2.312178 \mathrm{e}-18017-5.632278 \mathrm{e}-4$

$18000.59 c-1.2827 e-2$

$6000-1.24 \mathrm{e}-4$

$m \times 4: p$ 4j 180406012

m5 nlib=66c plib=04p elib=03e pnlib=25u gas=0 estep=3 \$ LANL soil

$1001-1.99977 \mathrm{e}-3$ 1002.24u $-2.3 \mathrm{e}-7$

$8016.24 \mathrm{u}-.5247228017-.001278$

$11023-.021$

$13027-.061$

$14028-.31819214029-.01615714030-.010651$

$19000-.029$

$26054-9.352 e-426056-1.468064 e-2 \quad 26057-3.3904 e-426058-4.512 e-5$

$m \times 5: p \circ 8 j 190394 j$

$c$ energy and thermal treatment

phys: $n 30$

phys:p $302 j-10$

phys:e 30

$c$ cut off

cut: $n$ j 0.1

cut: $p$ j 4.0

cut:e j 4.0

nps 1 e 6

c ctme 15

c peripheral

prdmp $3 j 2$

print

dbcn 7j 1 4j 458751

$c$ end of file 



\section{APPENDIX C}

\section{MCNPX INPUT FILES FOR NEUTRON AND}

PHOTON FLUX MAPS 



\section{Appendix C. MCNPX Input Files for Neutron and Photon Flux Maps}

\section{Neutron Field Input File:}

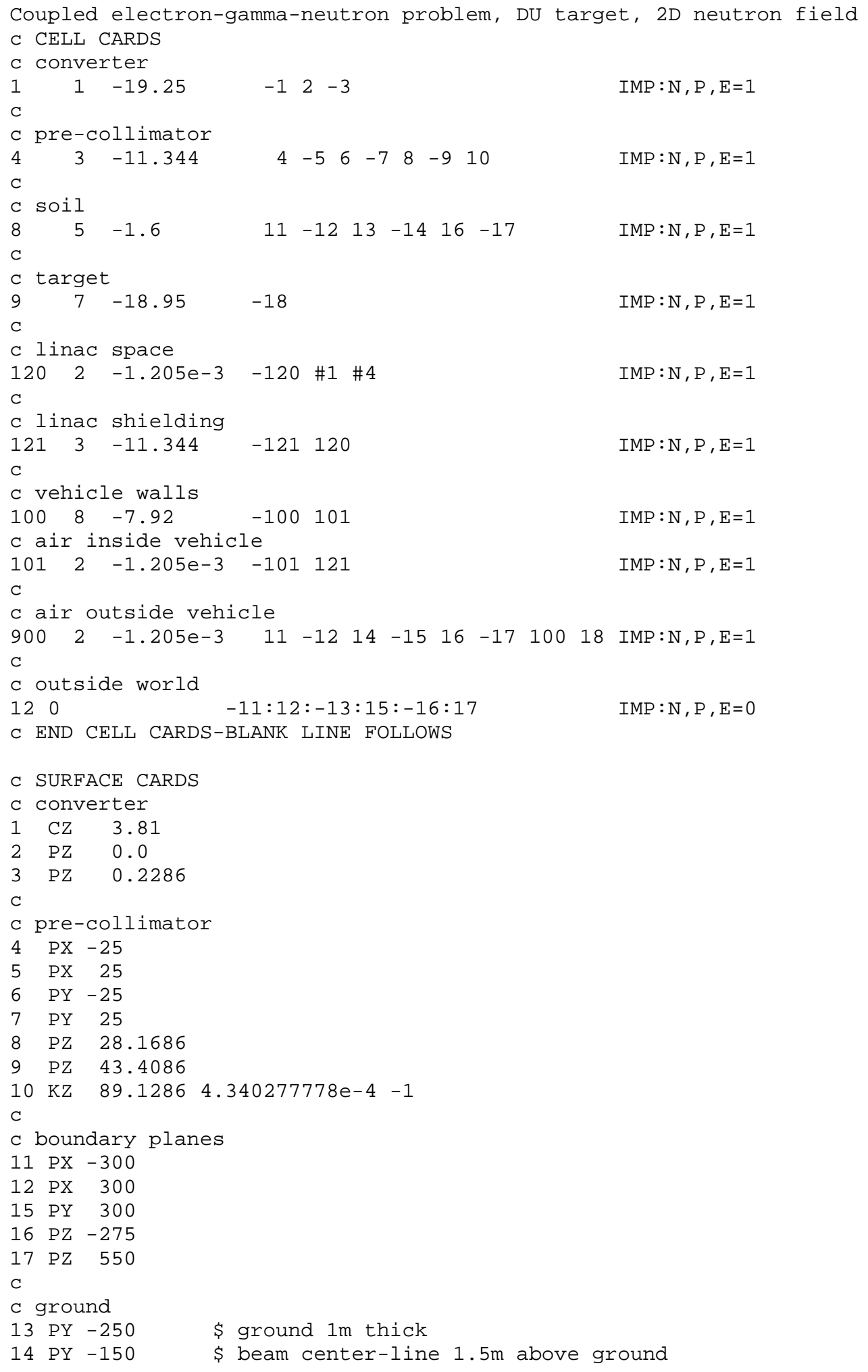




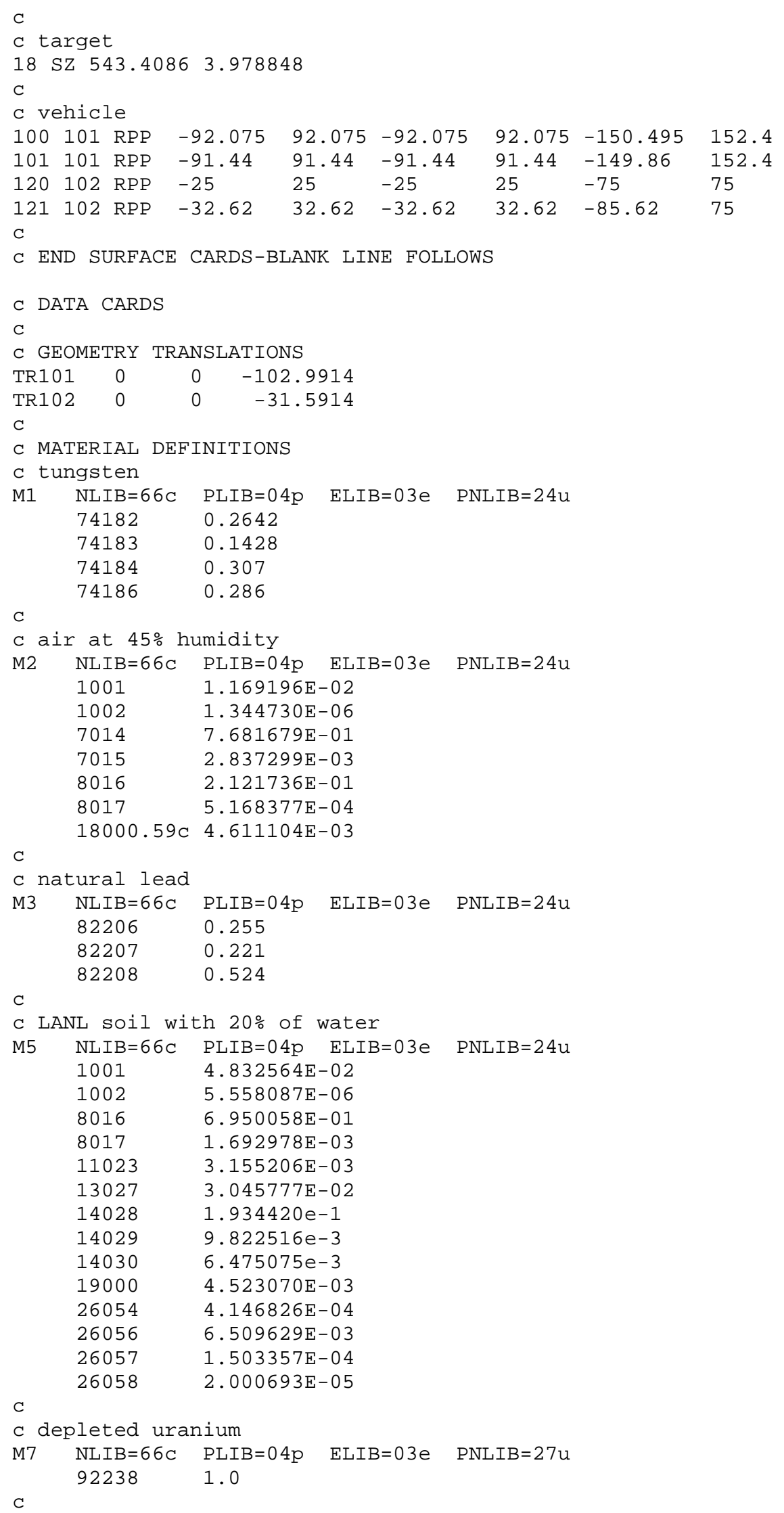




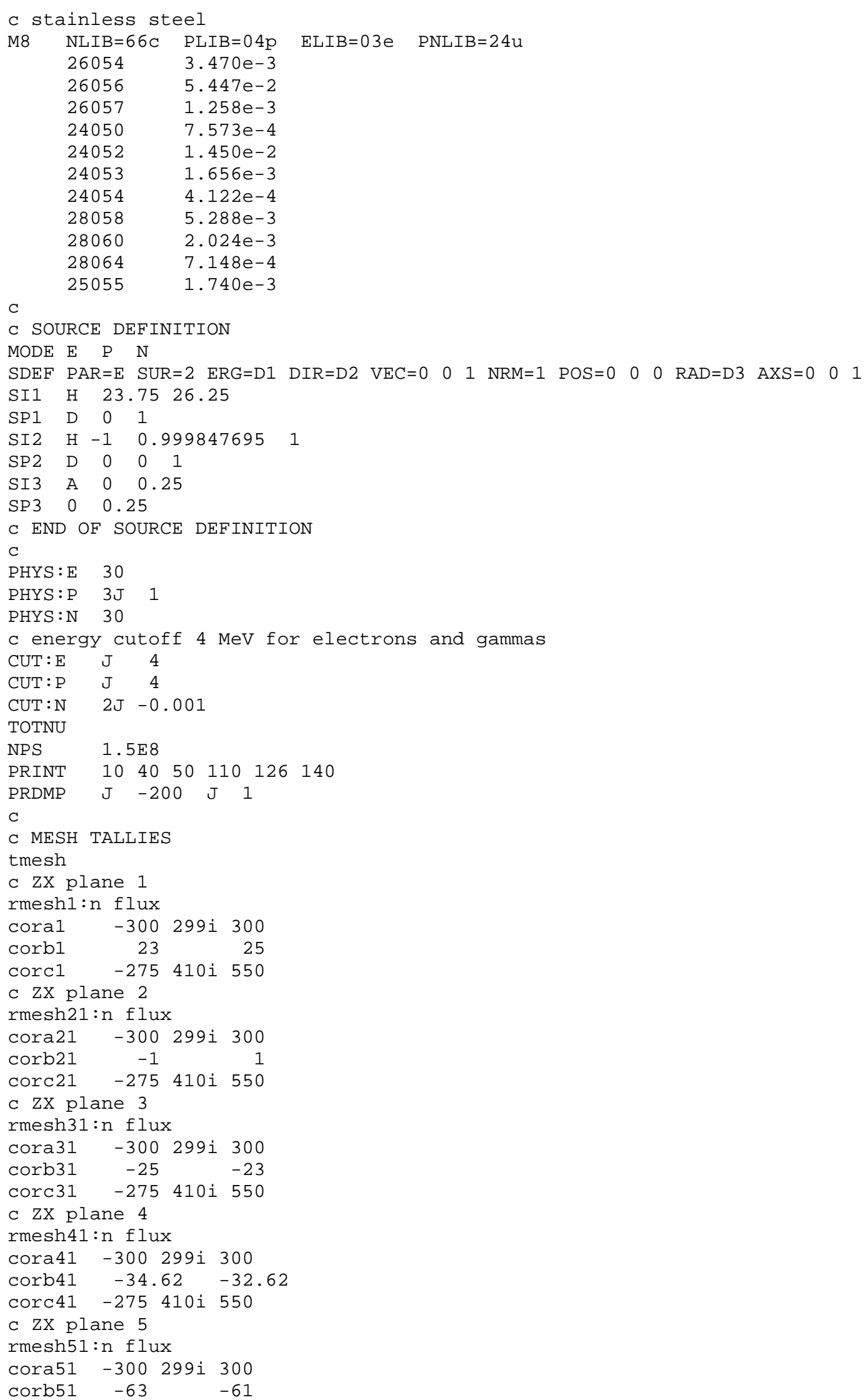




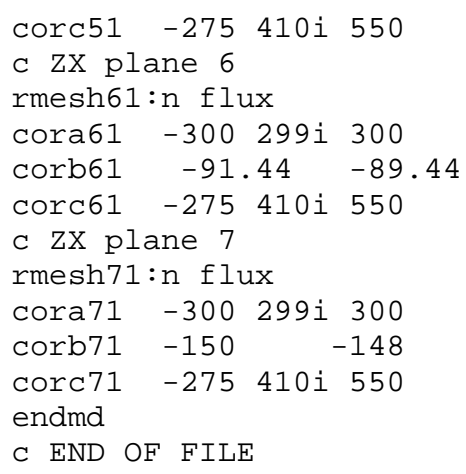

\section{Gamma Ray Field Input File:}

Coupled electron-gamma-neutron problem, DU target, 2D gamma field c CELL CARDS

C converter

$\begin{array}{lllllll}1 & 1 & -19.25 & -1 & 2 & -3 & \text { IMP : N, } P, E=1\end{array}$

C pre-collimator

$\begin{array}{lllllllllll}4 & 3 & -11.344 & 4 & -5 & 6 & -7 & 8 & -9 & 10 & \text { IMP : N, } P, E=1\end{array}$

C

c soil

$\begin{array}{llllllllll}8 & 5 & -1.6 & 11 & -12 & 13 & -14 & 16 & -17 & \text { IMP :N, } P, E=1\end{array}$

C

c target

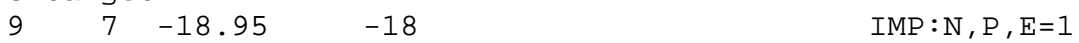

c linac space

$1202-1.205 e-3-120 \# 1 \# 4 \quad$ IMP : N, $P, E=1$

c

c linac shielding

$\begin{array}{lllll}121 & 3 & -11.344 & -121 & 120\end{array} \quad$ IMP : N, P, E=1

C

c vehicle walls

$\begin{array}{lllll}100 & 8 & -7.92 & -100 & 101\end{array} \quad$ IMP : N, $P, E=1$

c air inside vehicle

$\begin{array}{lllll}101 & 2 & -1.205 e-3 & -101 & 121\end{array}$

C

c air outside vehicle

$9002 \begin{array}{lllllllllll}9 & -1.205 e-3 & 11 & -12 & 14 & -15 & 16 & -17 & 100 & 18 & \text { IMP:N, } P, E=1\end{array}$

c

c outside world

$12 \odot-11: 12:-13: 15:-16: 17 \quad$ IMP $: N, P, E=0$

C END CELL CARDS-BLANK LINE FOLLOWS

c SURFACE CARDS

c converter

$1 \mathrm{CZ} \quad 3.81$

$P Z \quad 0.0$

$P Z \quad 0.2286$

pre-collimator

PX -25

PX 25

PY -25

PY 25

PZ 28.1686

$9 \mathrm{PZ} \quad 43.4086$

$10 \mathrm{KZ} 89.1286 \quad 4.340277778 \mathrm{e}-4-1$ 
$c$

C boundary planes

11 PX -300

$12 \mathrm{PX} 300$

15 PY 300

$16 \mathrm{PZ}-275$

$17 \mathrm{PZ} 550$

C

C ground

13 PY $-250 \quad \$$ ground $1 \mathrm{~m}$ thick

14 PY -150 \$ beam center-line $1.5 \mathrm{~m}$ above ground

c target

18 SZ $543.4086 \quad 3.978848$

$\mathrm{c}$

c vehicle

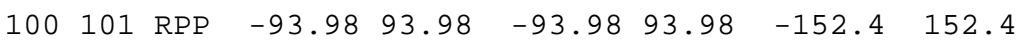

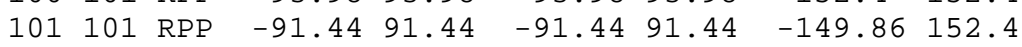

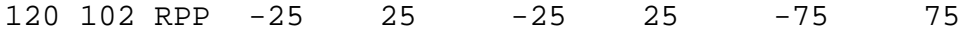

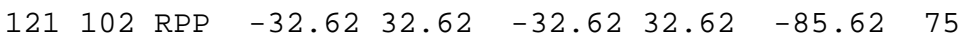

C

c END SURFACE CARDS-BLANK LINE FOLLOWS

C DATA CARDS

C

C GEOMETRY TRANSLATIONS

$\begin{array}{llll}\text { TR101 } & \odot & \odot & -102.9914\end{array}$

$\begin{array}{llll}\text { TR102 } & \odot & \odot & -31.5914\end{array}$

C

C MATERIAL DEFINITIONS

C tungsten

M1 NLIB=66c PLIB=๑4p ELIB=03e PNLIB=24u

$74182 \quad 0.2642$

$74183 \quad 0.1428$

$74184 \quad 0.307$

C

$74186 \quad 0.286$

C air at $45 \%$ humidity

M2 NLIB $=66 \mathrm{c} \quad \mathrm{PLIB}=04 \mathrm{p} \quad \mathrm{ELIB}=03 \mathrm{e} \quad \mathrm{PNLIB}=24 \mathrm{u}$

$1001 \quad 1.169196 \mathrm{E}-02$

$1002 \quad 1.344730 \mathrm{E}-06$

$7014 \quad 7.681679 \mathrm{E}-01$

$7015 \quad 2.837299 \mathrm{E}-03$

$8016 \quad 2.121736 \mathrm{E}-01$

$8017 \quad 5.168377 \mathrm{E}-04$

$18000.59 \mathrm{C} \quad 4.611104 \mathrm{E}-03$

$C$ natural lead

M3 NLIB=66c PLIB $=04 p \quad E L I B=03 e \quad P N L I B=24 u$

$82206 \quad 0.255$

$82207 \quad 0.221$

$82208 \quad 0.524$

C

C LANL soil with $20 \%$ of water

M5 NLIB $=66 \mathrm{c} \quad \mathrm{PLIB}=04 \mathrm{p} \quad \mathrm{ELIB}=03 \mathrm{e} \quad \mathrm{PNLIB}=24 \mathrm{u}$

$1001 \quad 4.832564 \mathrm{E}-02$

$1002 \quad 5.558087 \mathrm{E}-06$

$8016 \quad 6.950058 \mathrm{E}-01$

$8017 \quad 1.692978 \mathrm{E}-03$

$11023 \quad 3.155206 \mathrm{E}-03$

$13027 \quad 3.045777 \mathrm{E}-02$

$14028 \quad 1.934420 \mathrm{e}-1$

$140299.822516 \mathrm{e}-3$ 


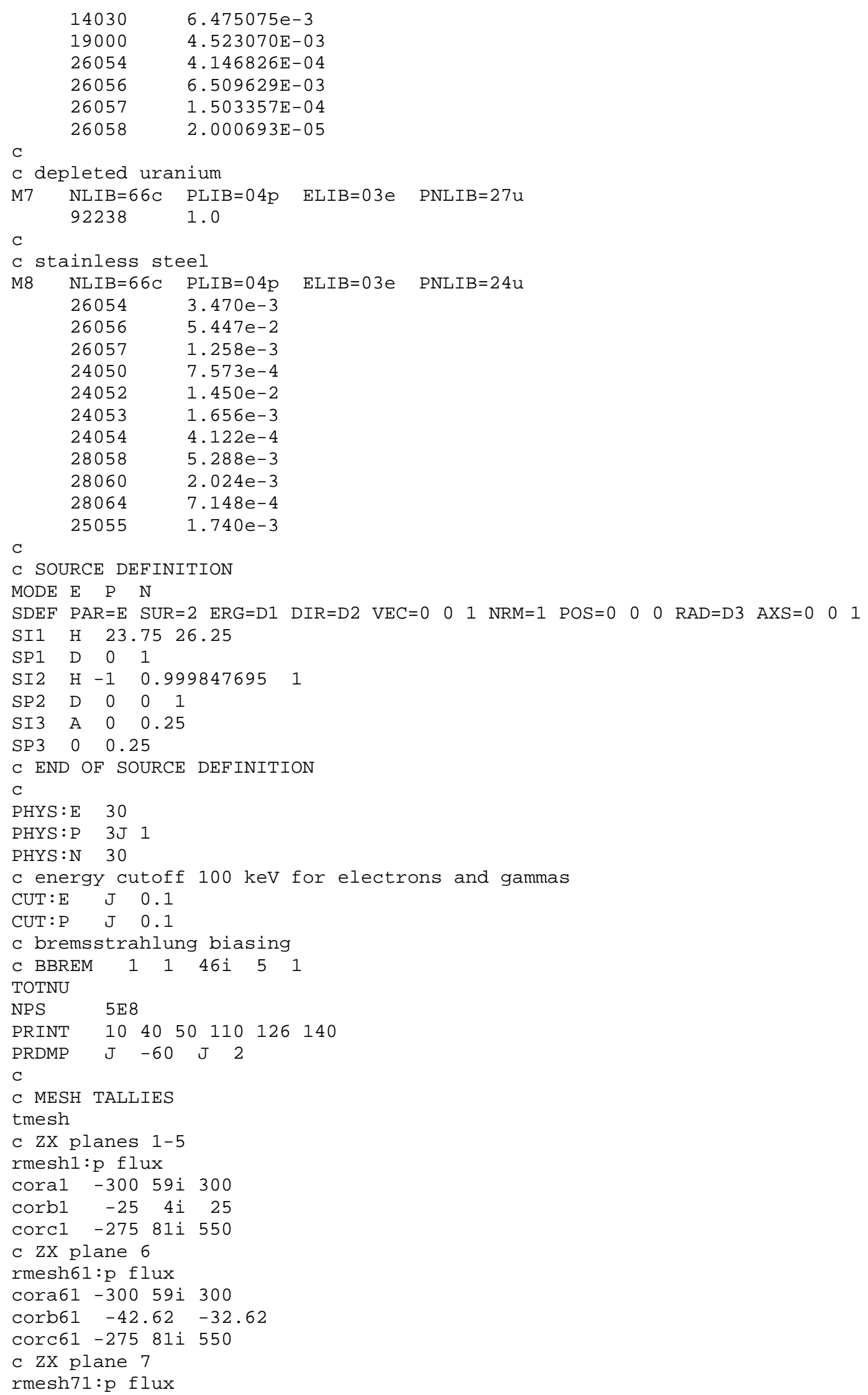


cora71 $-300 \quad 59 i \quad 300$

$\begin{array}{lll}\text { corb71 } & -67 & -57\end{array}$

corc71 -275 81i 550

c ZX plane 8

rmesh81:p flux

cora81 -300 59i 300

corb81 $-91.44 \quad-81.44$

corc81 -275 81i 550

c ZX planes 9-14

rmesh91:p flux

cora91 -300 59i 300

corb91 $-150 \quad 5 i-93.98$

corc91 -275 81i 550

endmd

c END OF FILE 

APPENDIX D

NEUTRON AND PHOTON FLUENCE MAP ERRORS 

Appendix D. Neutron and Photon Fluence Map Errors

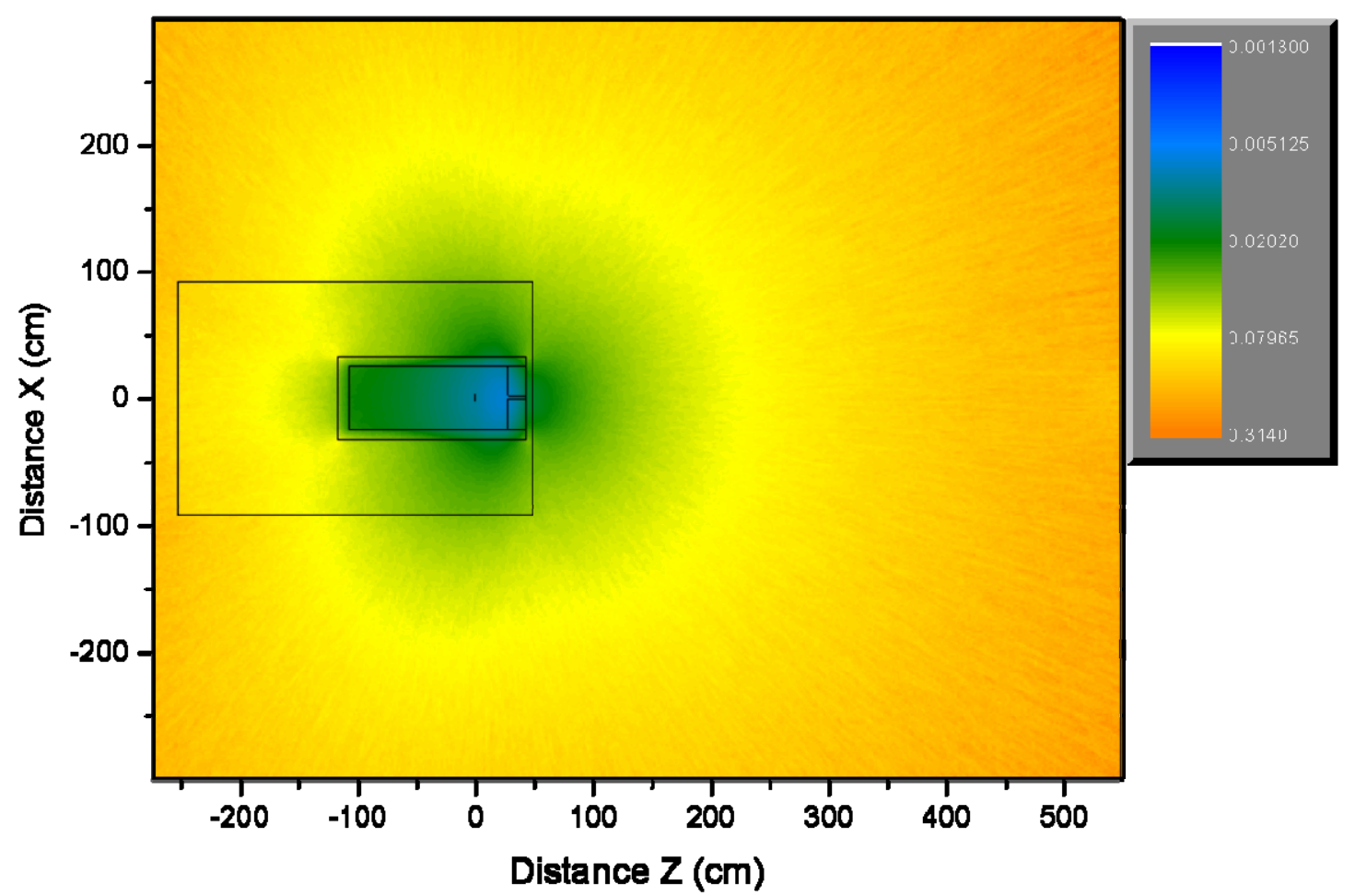

Figure D-1. Relative errors for the neutron fluence map shown in Figure 21.

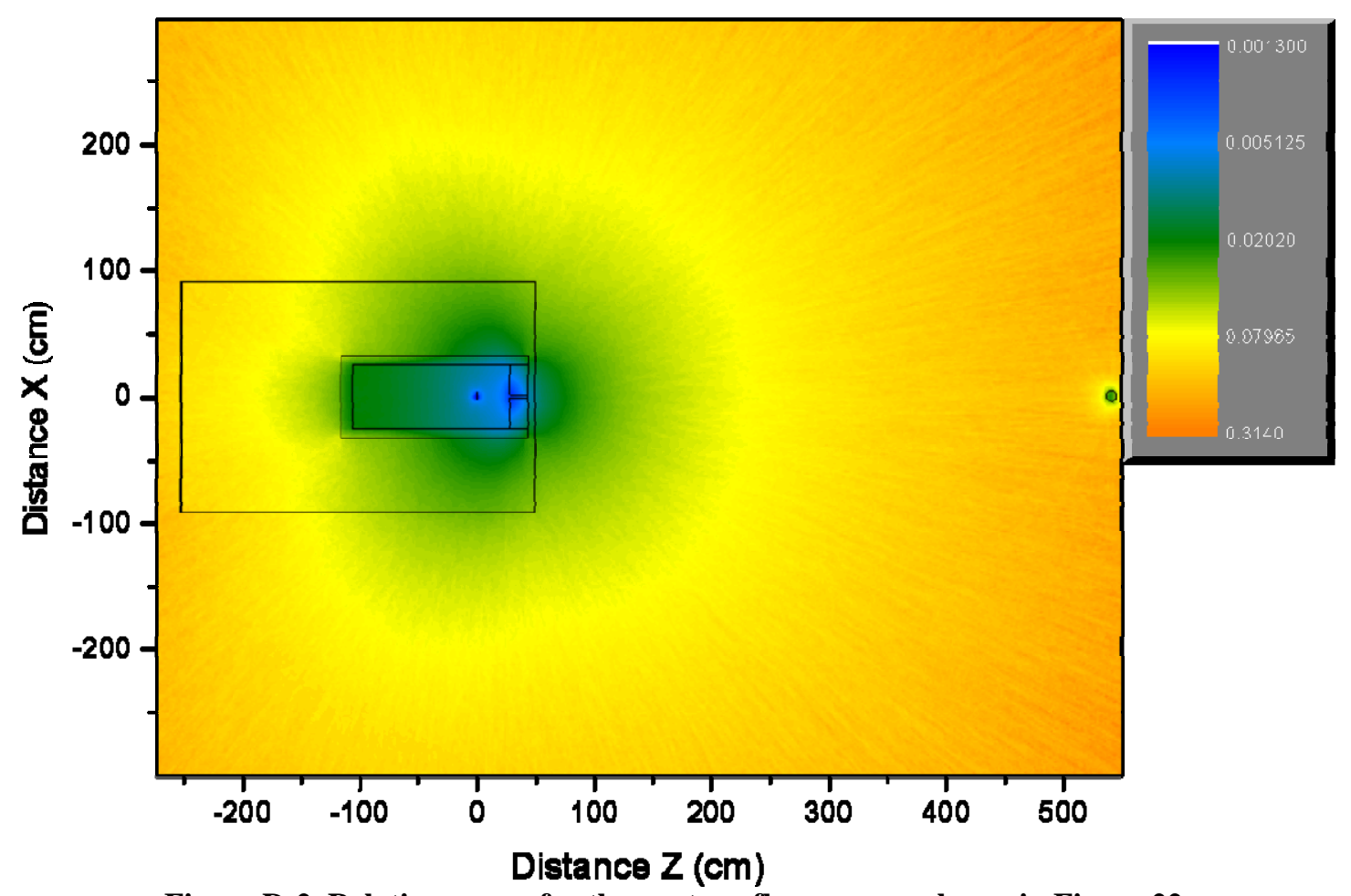

Figure D-2. Relative errors for the neutron fluence map shown in Figure 22. 


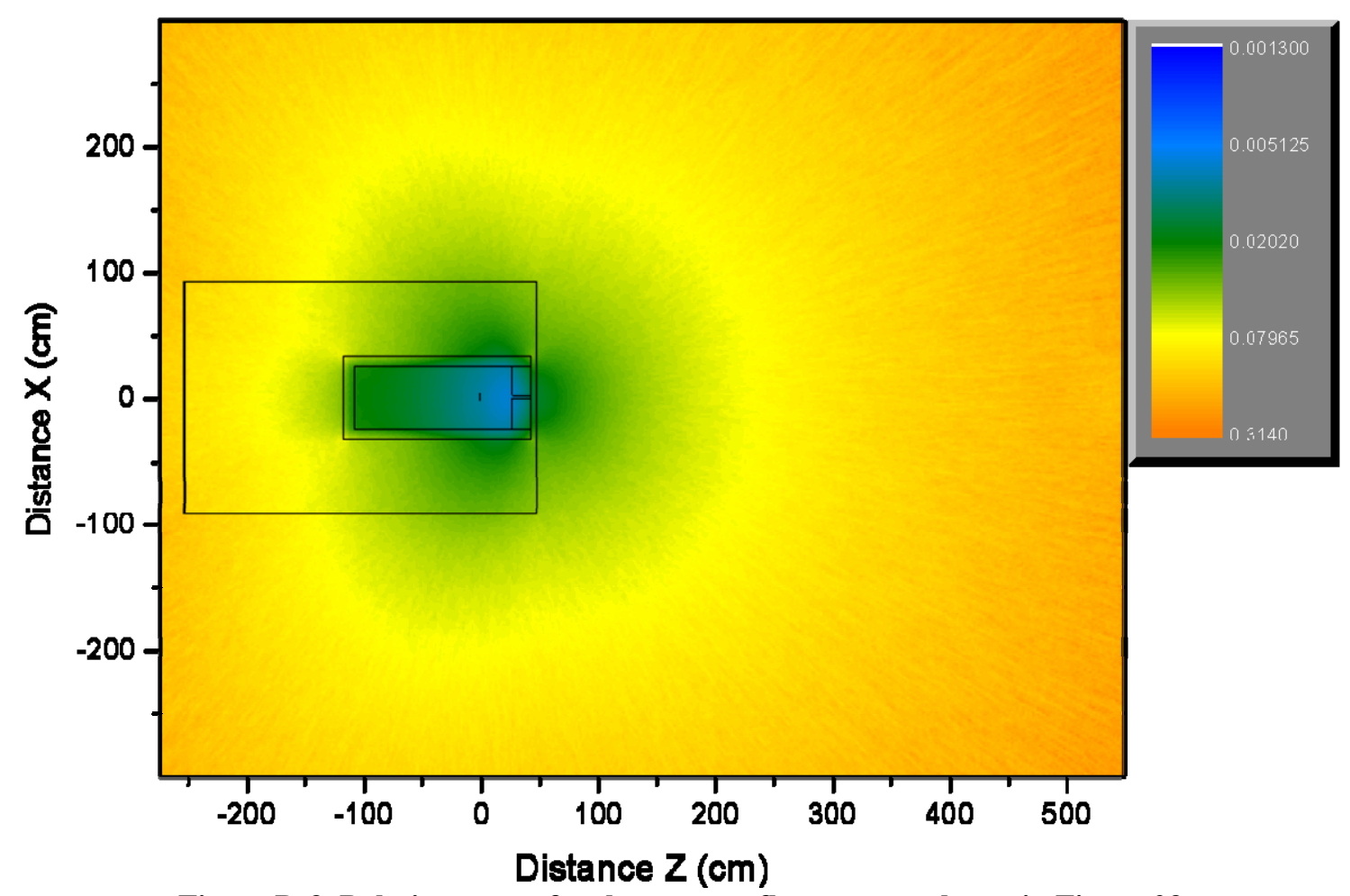

Figure D-3. Relative errors for the neutron fluence map shown in Figure 23.

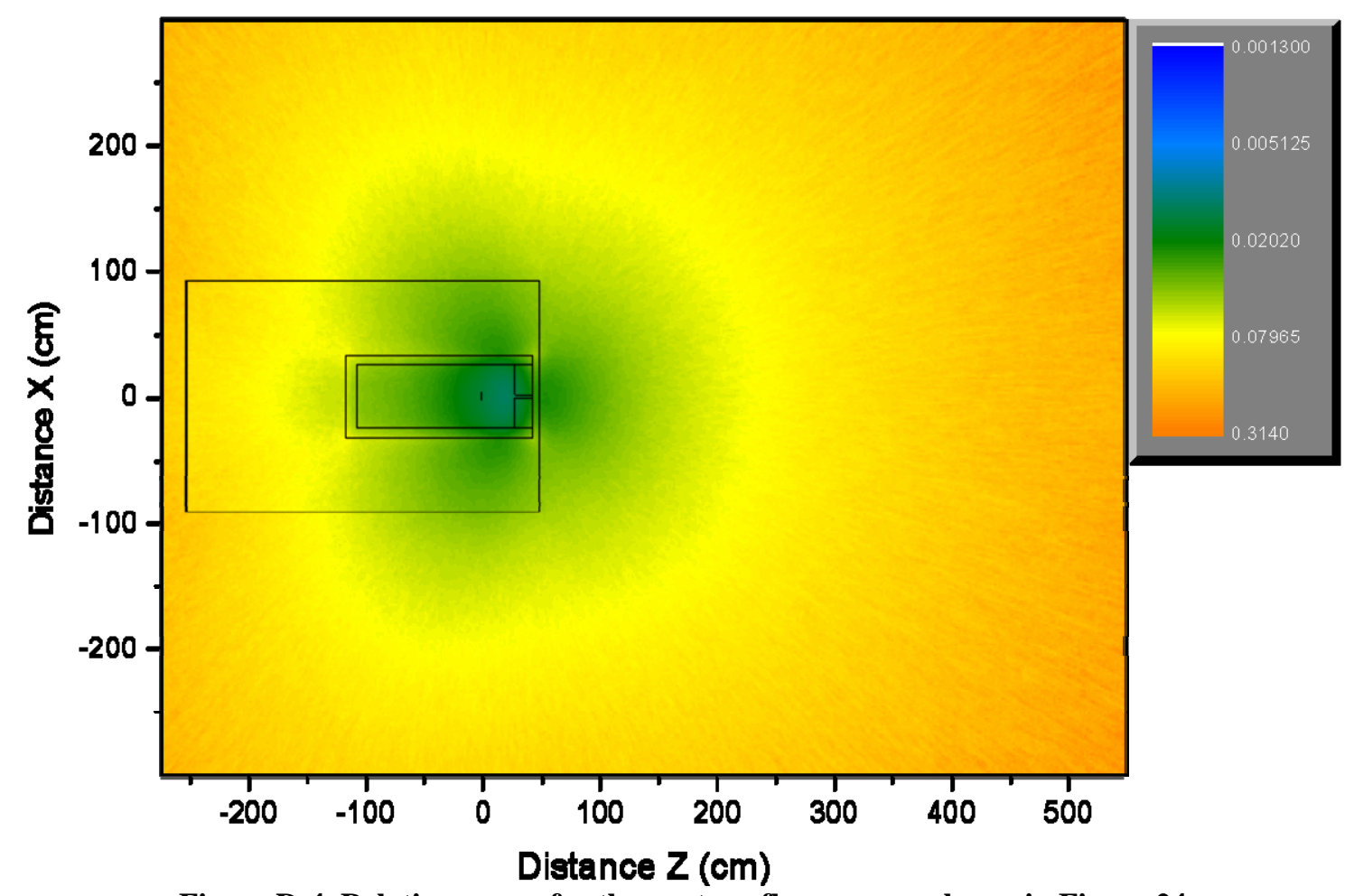

Figure D-4. Relative errors for the neutron fluence map shown in Figure 24. 


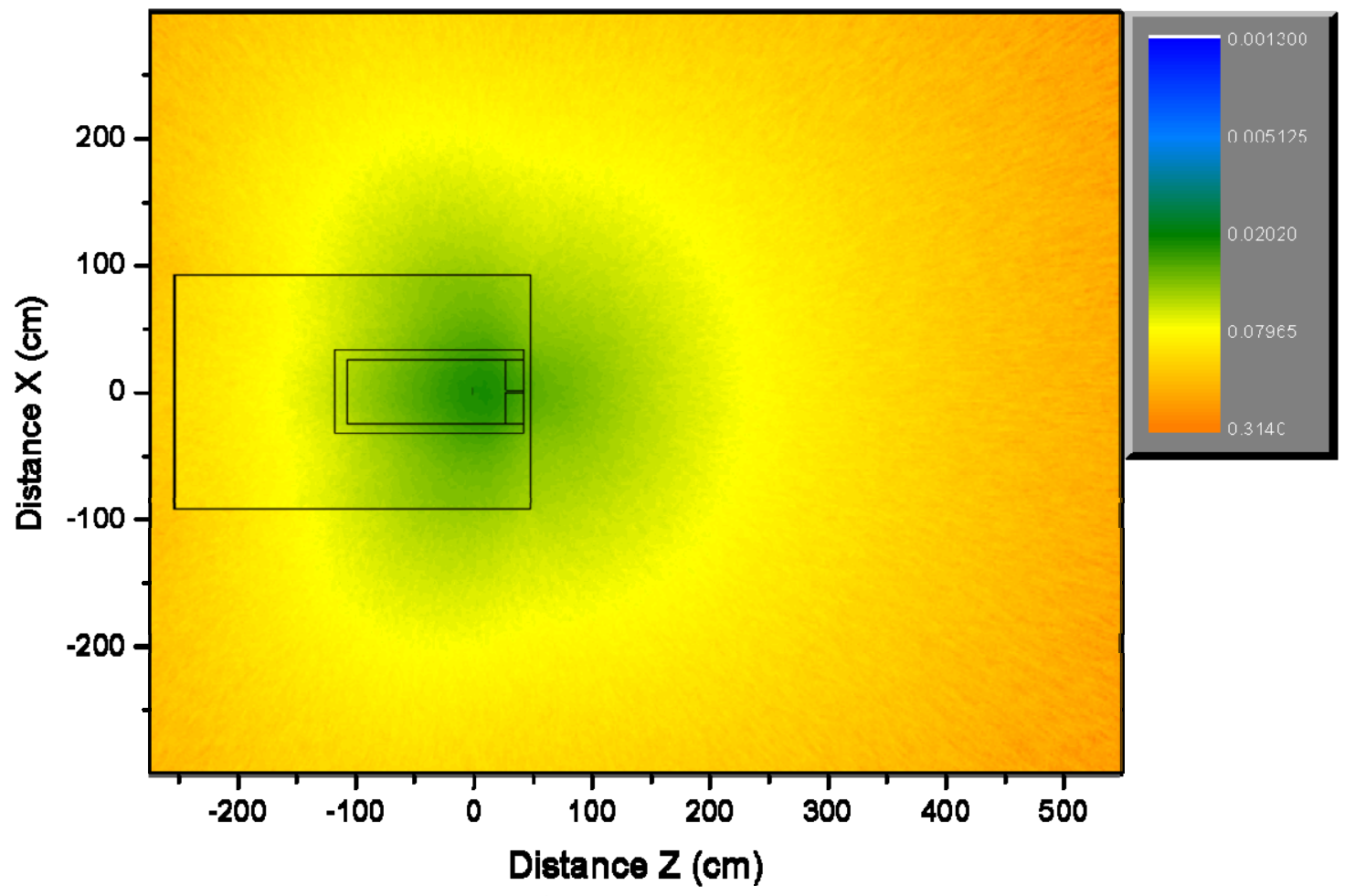

Figure D-5. Relative errors for the neutron fluence map shown in Figure 25.

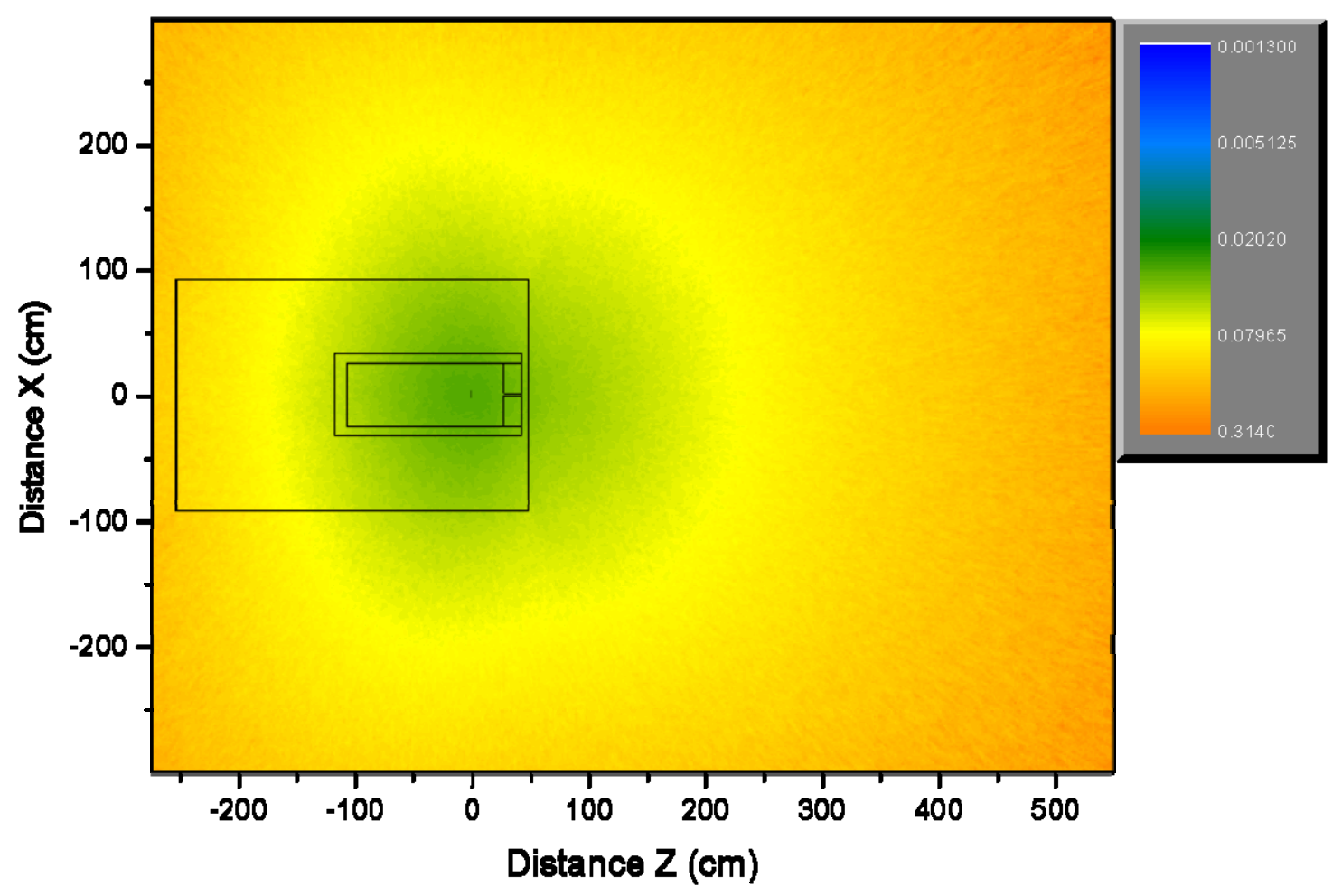

Figure D-6. Relative errors for the neutron fluence map shown in Figure 26. 


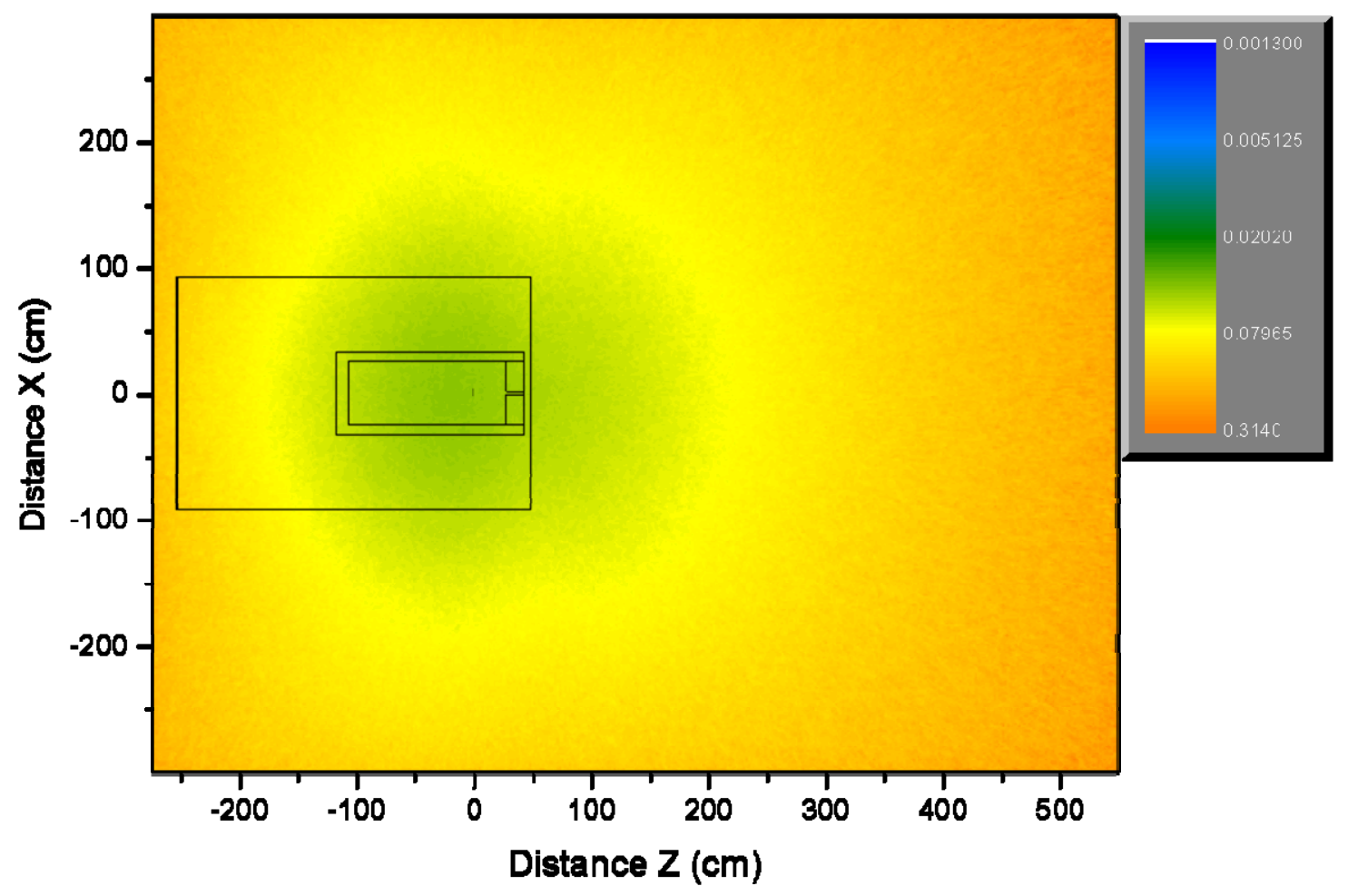

Figure D-7. Relative errors for the neutron fluence map shown in Figure 27.

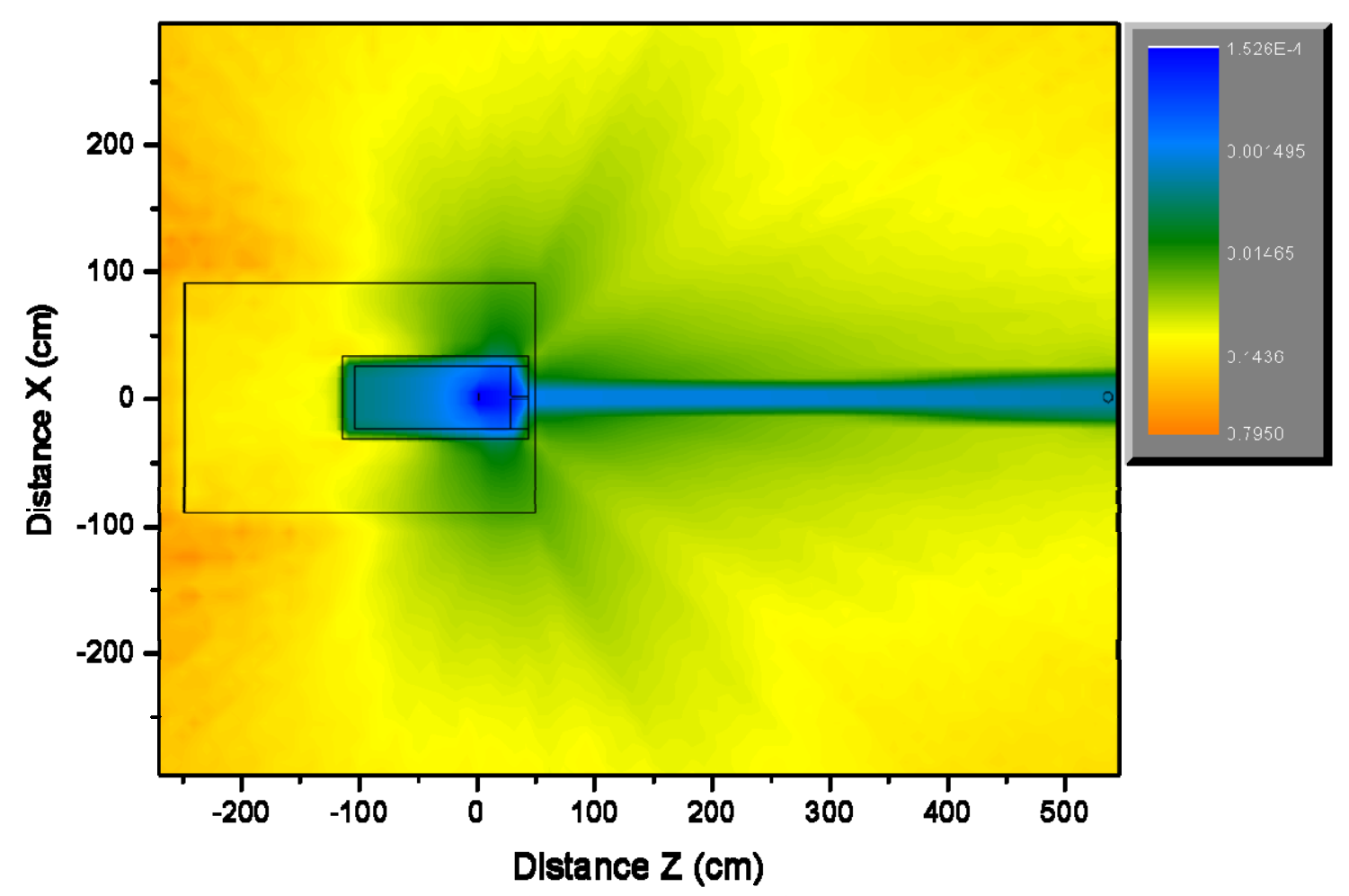

Figure D-8. Relative errors for the gamma ray fluence map shown in Figure 28. 


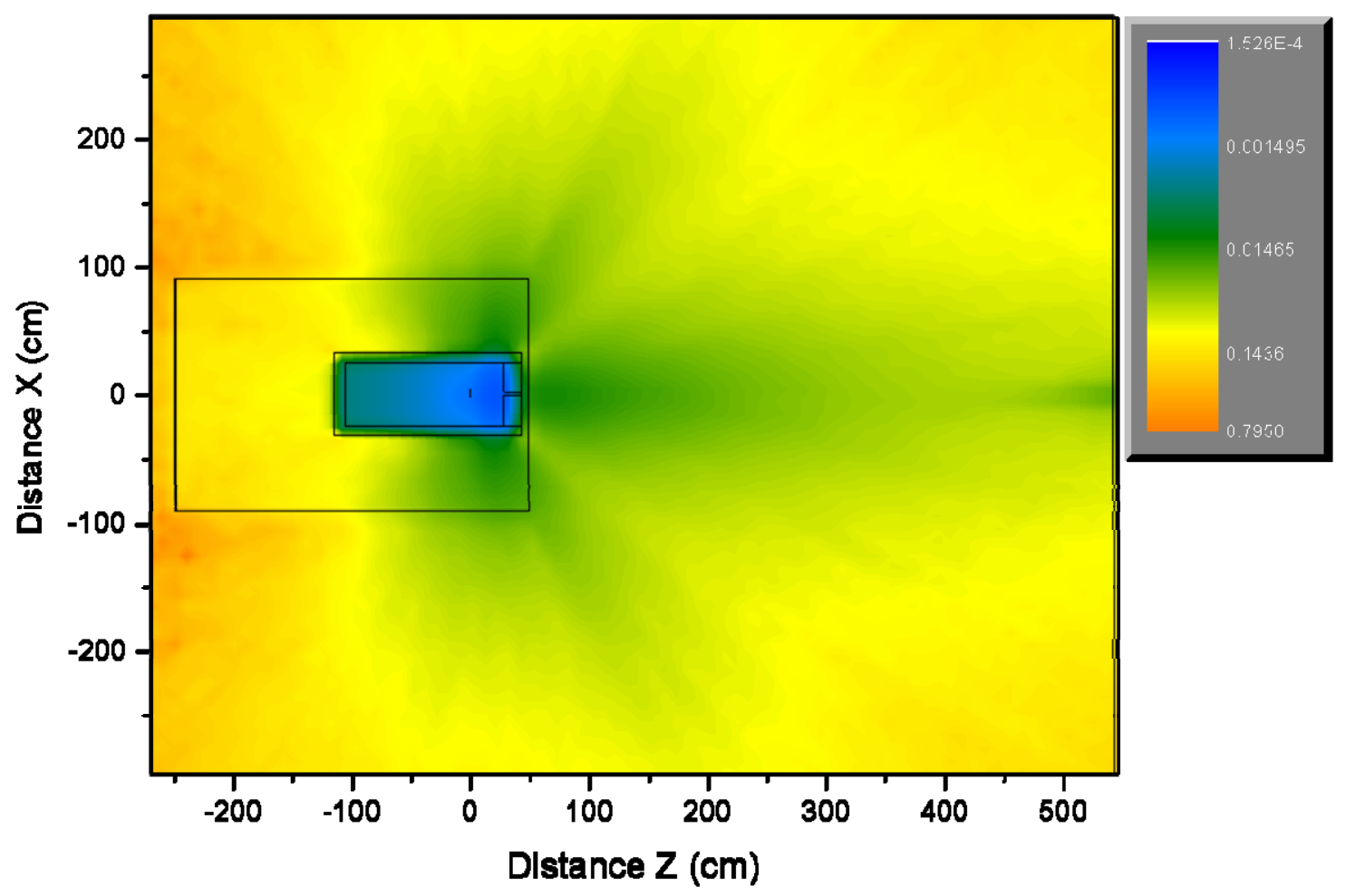

Figure D-9. Relative errors for the gamma ray fluence map shown in Figure 29.

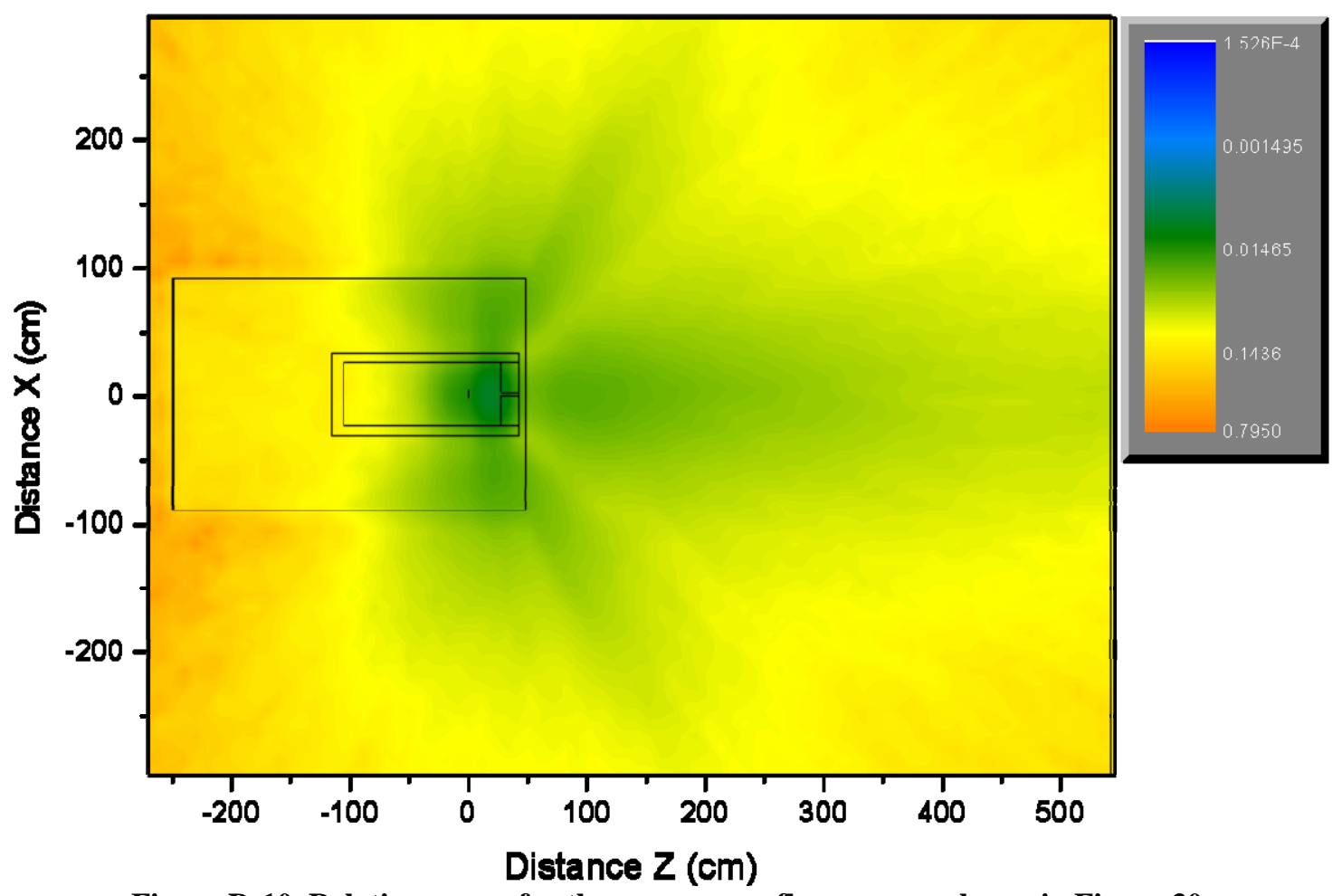

Figure D-10. Relative errors for the gamma ray fluence map shown in Figure 30. 


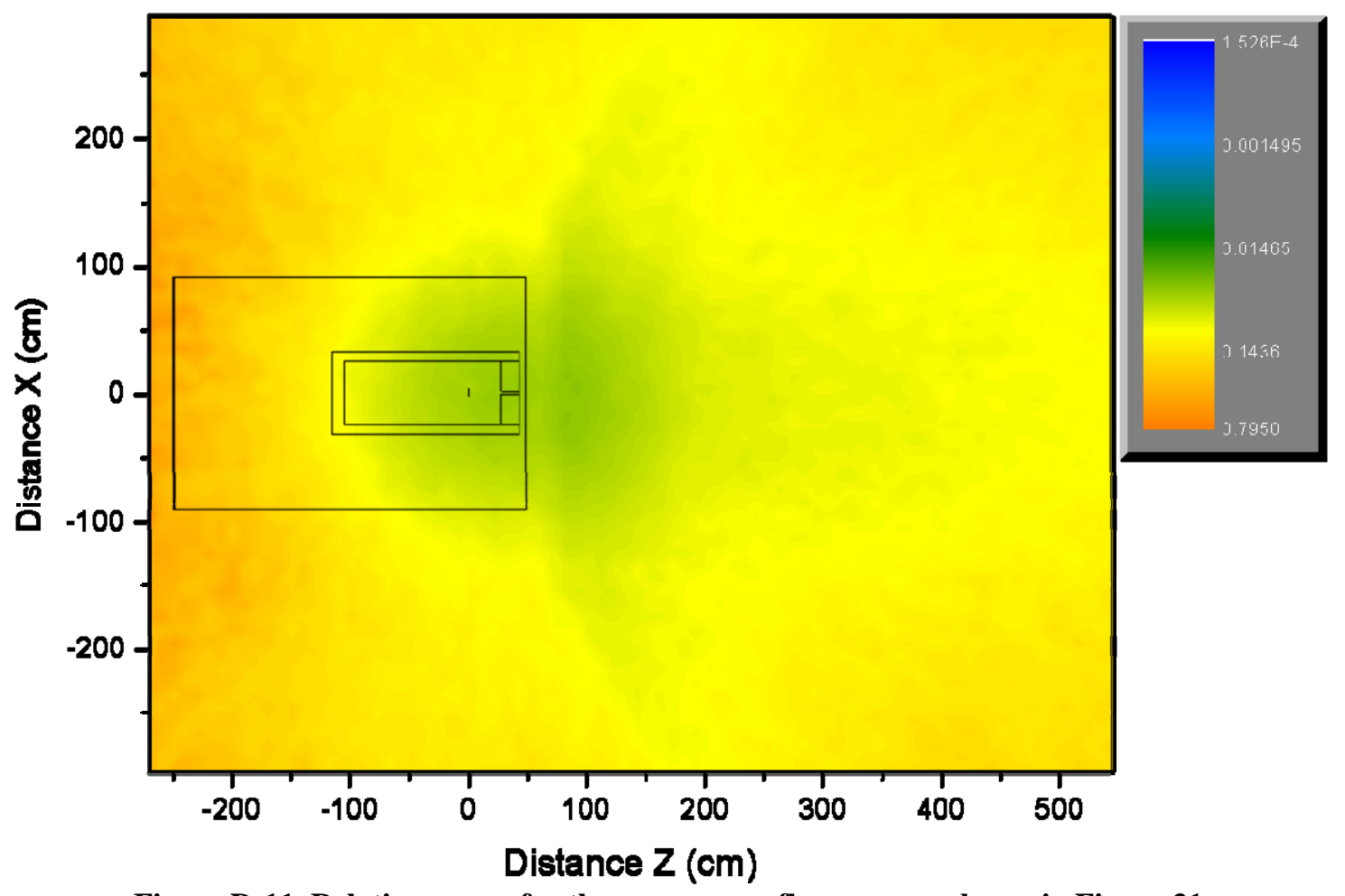

Figure D-11. Relative errors for the gamma ray fluence map shown in Figure 31. 
APPENDIX E

MCNPX INPUT FILE FOR TIME-ENERGY DEPENDENCE 



\section{Appendix E. MCNPX Input File for Time-Energy Dependence}

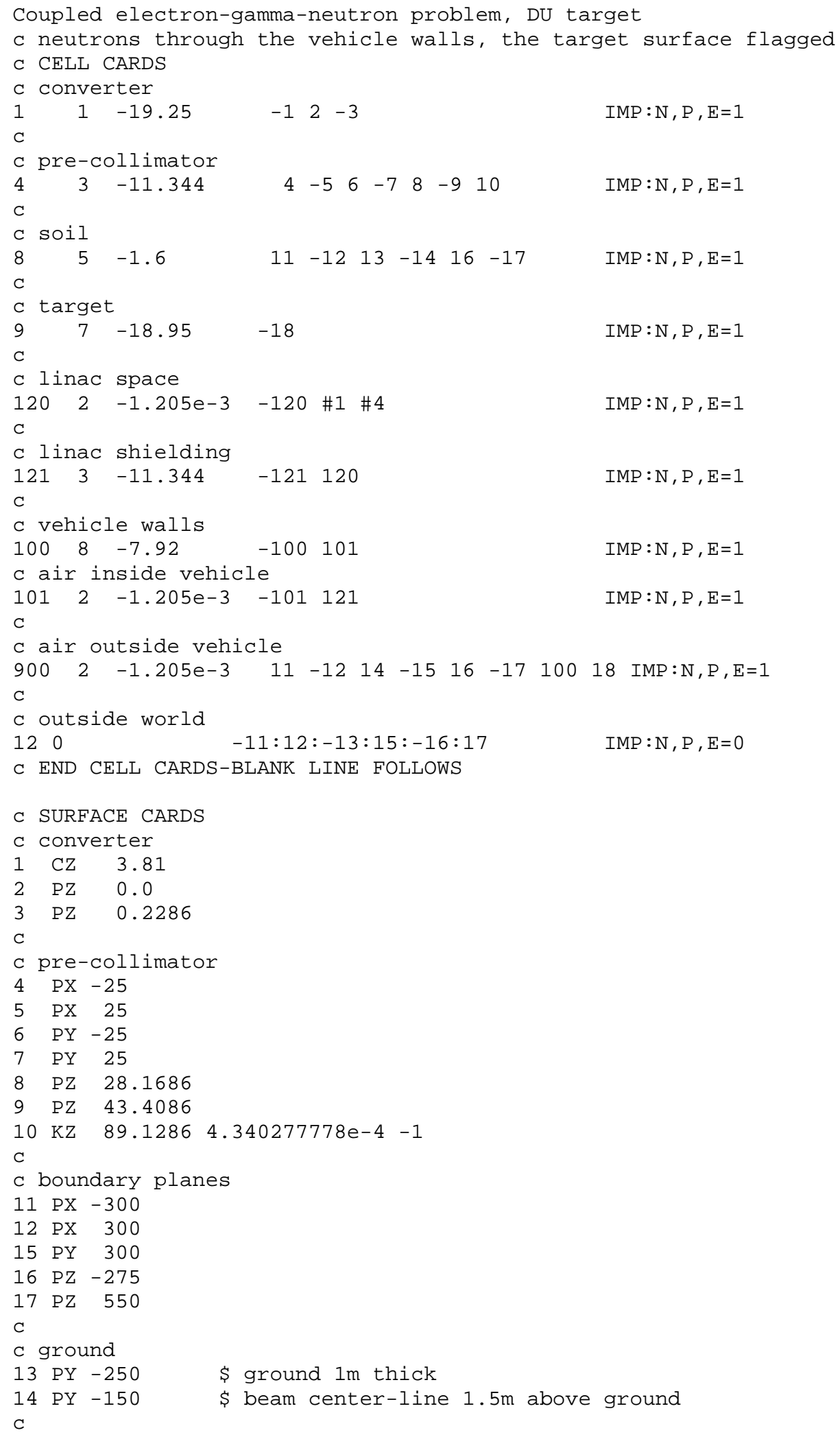




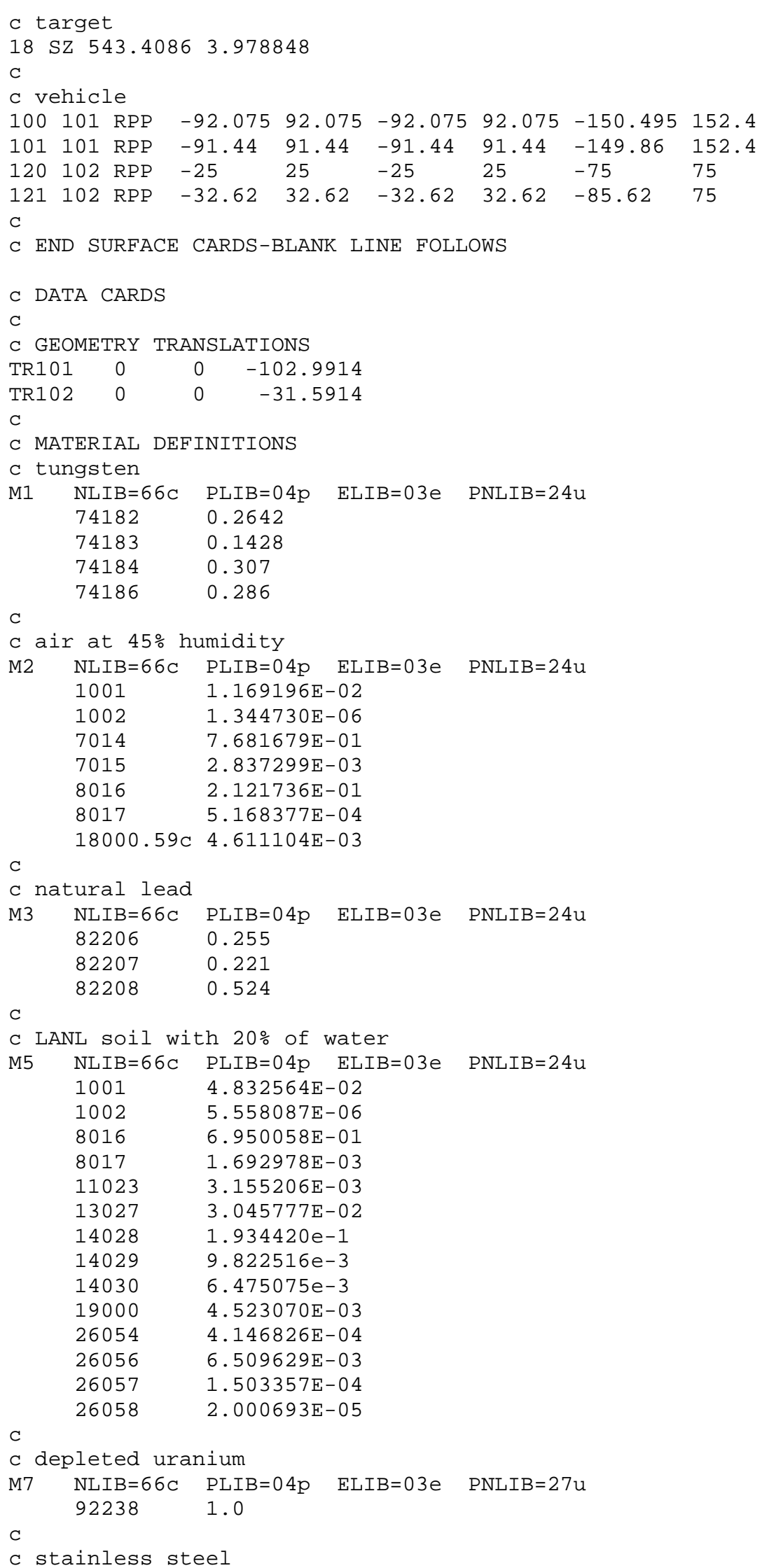




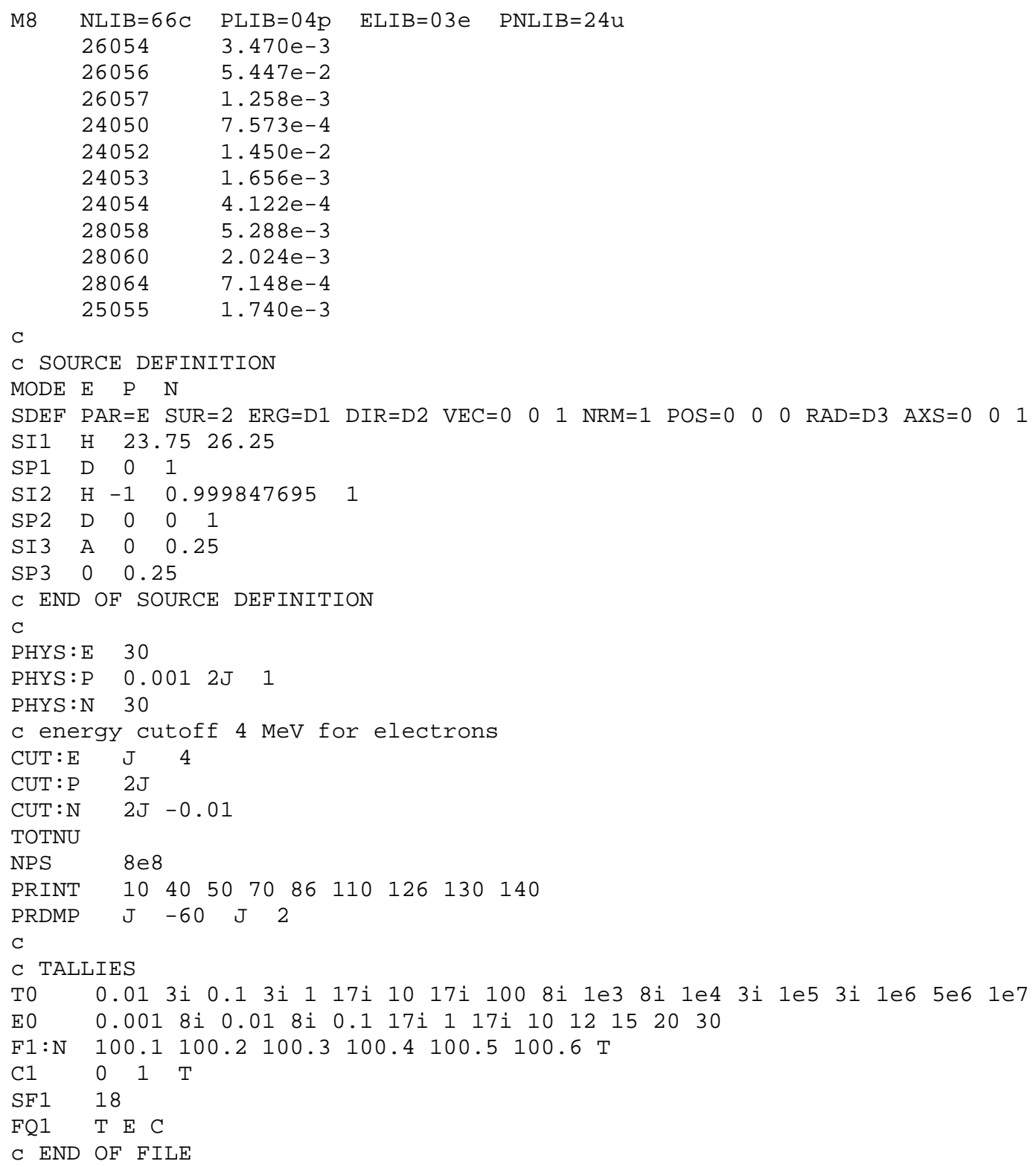



APPENDIX F

MCNP-POLIMI INPUT FILES FOR PHOTONUCLEAR SOURCES 



\section{Appendix F. MCNP-PoliMi Input Files for Photonuclear Sources}

\section{DU Target:}

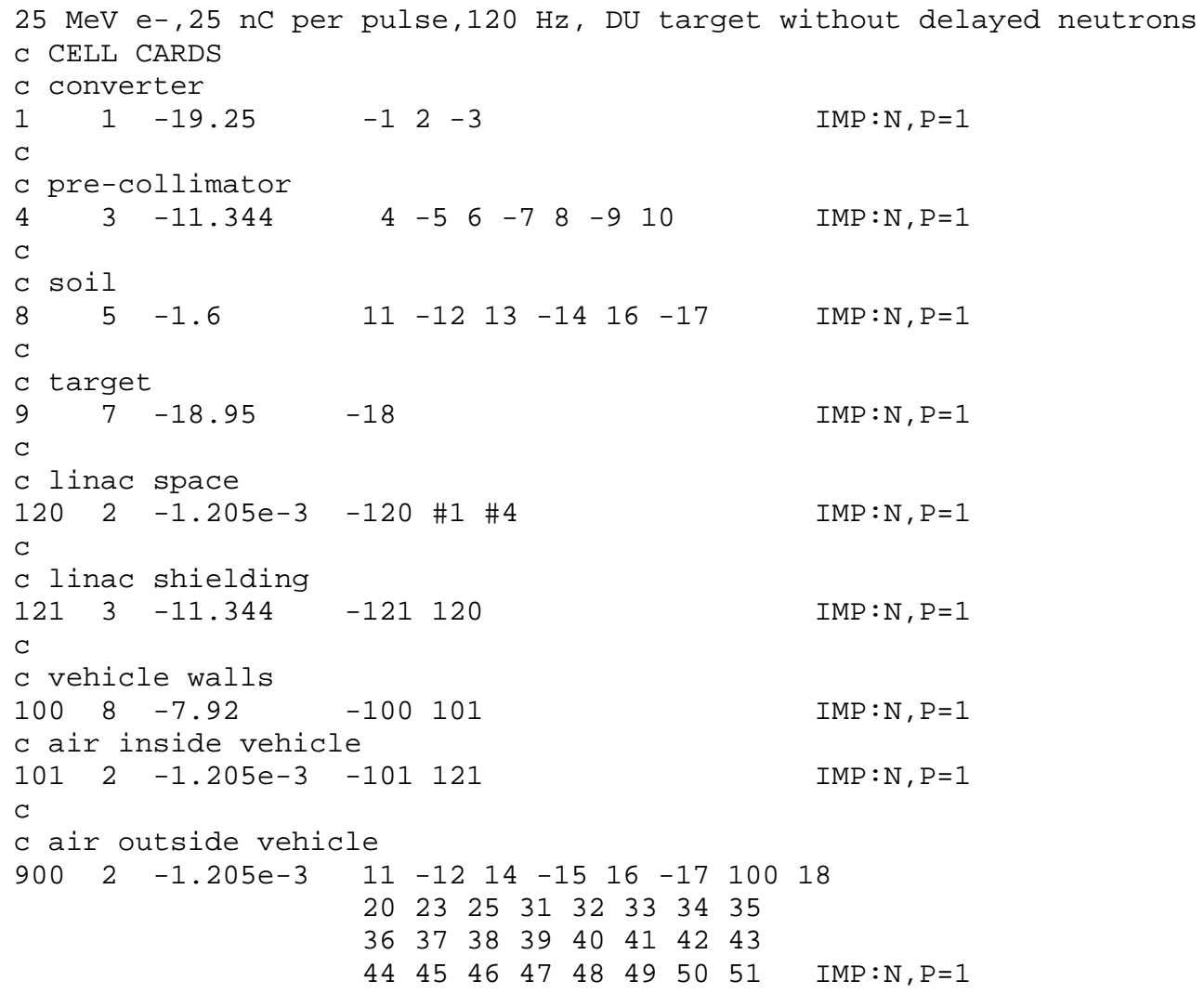




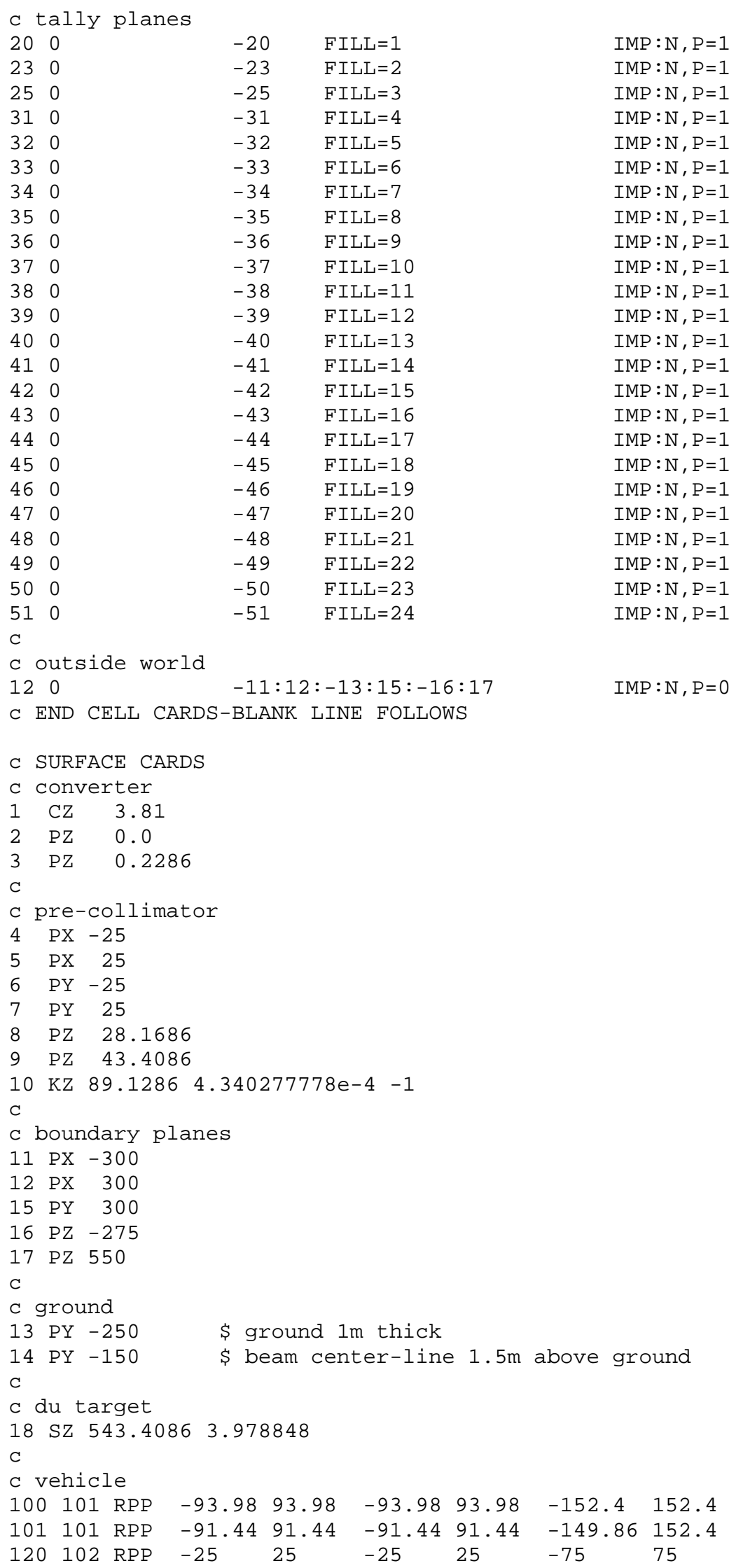




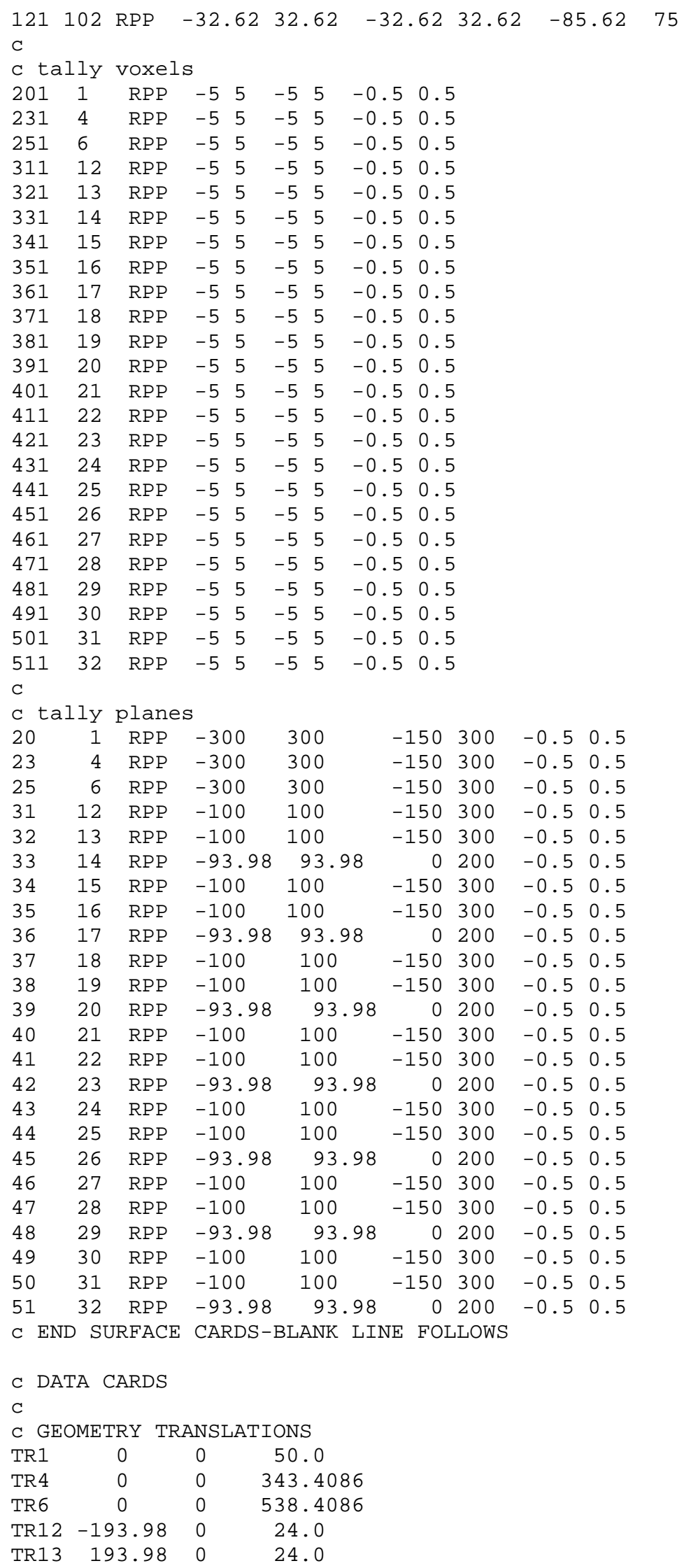




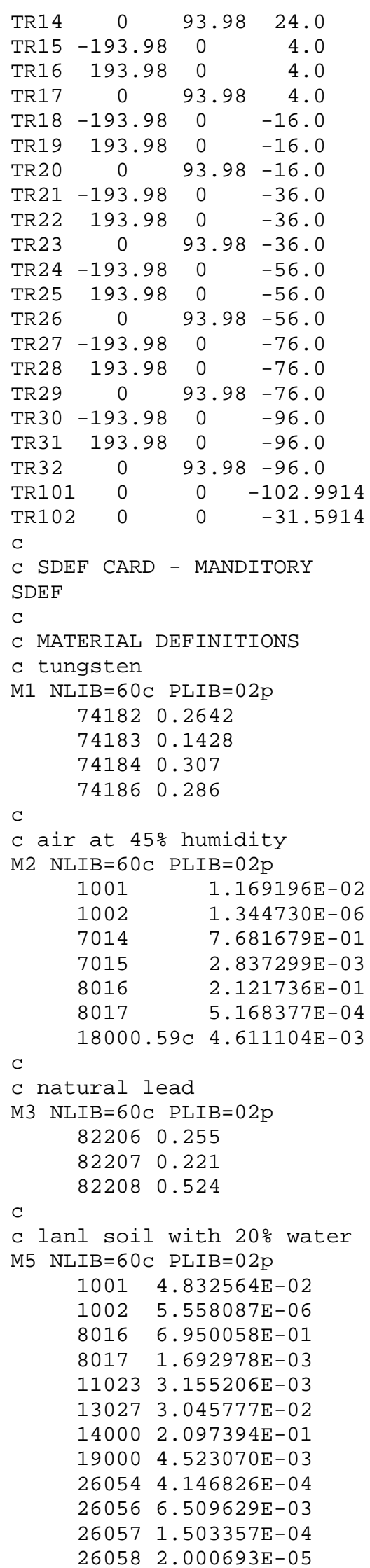




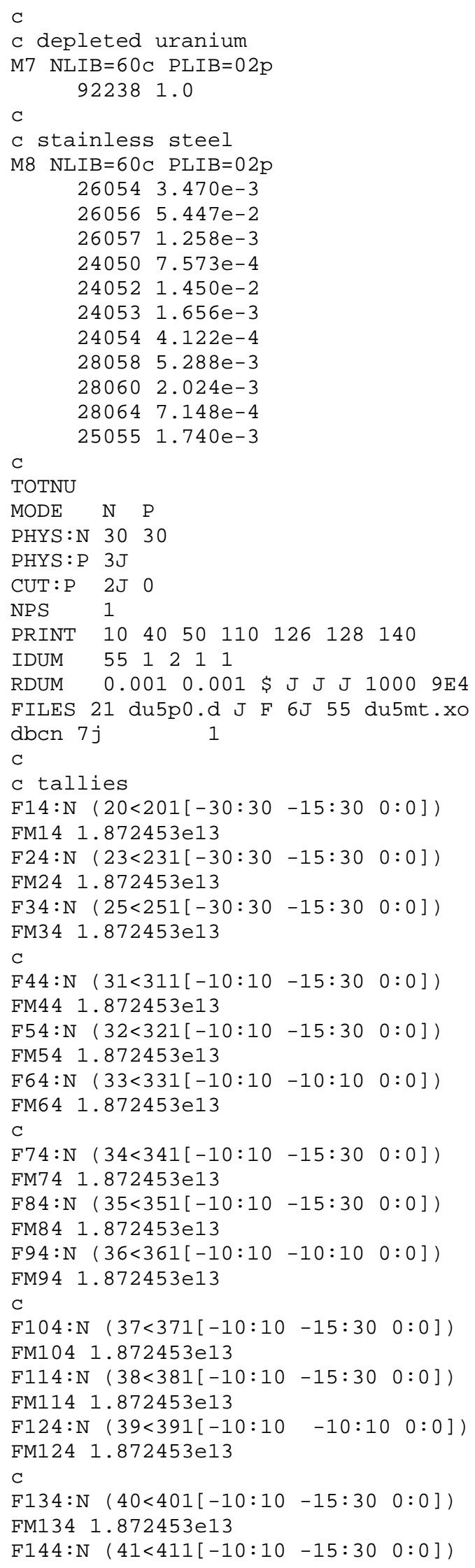


FM144 1.872453e13

F154:N $(42<421[-10: 10-10: 10 \quad 0: 0])$

FM154 1.872453e13

$\mathrm{C}$

F164:N $(43<431[-10: 10 \quad-15: 30 \quad 0: 0])$

FM164 1.872453e13

F174:N $\left(44<441\left[\begin{array}{llll}-10: 10 & -15: 30 & 0: 0\end{array}\right)\right.$

FM174 $1.872453 \mathrm{e} 13$

F184:N $(45<451[-10: 10-10: 10 \quad 0: 0])$

FM184 1.872453e13

$\mathrm{C}$

F194:N $(46<461[-10: 10 \quad-15: 30 \quad 0: 0])$

FM194 1.872453e13

F204:N $(47<471[-10: 10-15: 30 \quad 0: 0])$

FM204 $1.872453 \mathrm{e} 13$

F214:N $(48<481[-10: 10-10: 10 \quad 0: 0])$

FM214 1.872453e13

$\mathrm{C}$

F224:N $(49<491[-10: 10-15: 30 \quad 0: 0])$

FM224 1.872453e13

F234: N $(50<501[-10: 10-15: 30 \quad 0: 0])$

FM234 1.872453e13

F244: N $(51<511[-10: 10-10: 10 \quad 0: \odot])$

FM244 $1.872453 \mathrm{e} 13$

$\mathrm{C}$

C

F254:P $(20<201[-30: 30-15: 30 \quad 0: 0])$

FM254 1.872453e13

F264:P $(23<231[-30: 30-15: 30 \quad 0: 0])$

FM264 1.872453e13

F274:P $\left(25<251\left[\begin{array}{llll}-30: 30 & -15: 30 & 0: \odot\end{array}\right)\right.$

FM274 $1.872453 \mathrm{e} 13$

$\mathrm{C}$

F284:P $(31<311[-10: 10 \quad-15: 30 \quad \odot: \odot])$

FM284 1.872453e13

F294:P $(32<321[-10: 10 \quad-15: 30 \quad 0: 0])$

FM294 1.872453e13

F304:P $(33<331[-10: 10 \quad-10: 10 \quad 0: 0])$

FM304 1.872453e13

$\mathrm{C}$

F314:P $(34<341[-10: 10 \quad-15: 30 \quad \odot: 0])$

FM314 1.872453e13

F324:P $(35<351[-10: 10-15: 30 \quad 0: 0])$

FM324 1.872453e13

F334:P $(36<361[-10: 10 \quad-10: 10 \quad 0: 0])$

FM334 $1.872453 \mathrm{e} 13$

$\mathrm{C}$

F344:P $(37<371[-10: 10 \quad-15: 30 \quad \odot: \odot])$

FM344 1.872453e13

F354:P $\left(38<381\left[\begin{array}{llll}-10: 10 & -15: 30 & 0: 0\end{array}\right)\right.$

FM354 $1.872453 \mathrm{e} 13$

F364:P $(39<391[-10: 10 \quad-10: 10 \quad 0: 0])$

FM364 1.872453e13

$\mathrm{C}$

F374:P $(40<401[-10: 10-15: 30 \quad 0: \odot])$

FM374 1.872453e13

F384:P $(41<411[-10: 10-15: 30 \quad 0: 0])$

FM384 1.872453e13

F394:P $(42<421[-10: 10 \quad-10: 10 \quad 0: 0])$

FM394 $1.872453 \mathrm{e} 13$

$\mathrm{C}$

F404:P $(43<431[-10: 10-15: 30 \quad 0: \odot])$

FM404 $1.872453 \mathrm{e} 13$ 


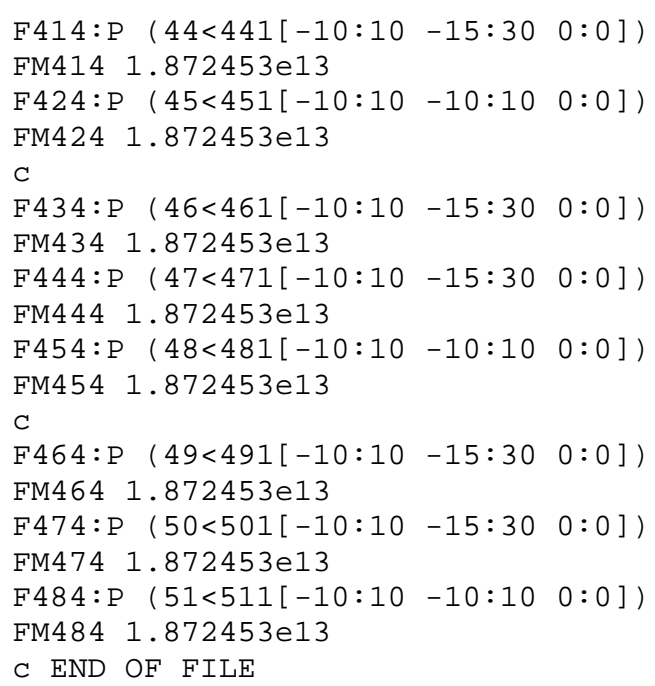

\section{HEU Target:}

$25 \mathrm{MeV}$ e-,25 nC per pulse, $120 \mathrm{~Hz}$, HEU target without delayed neutrons 


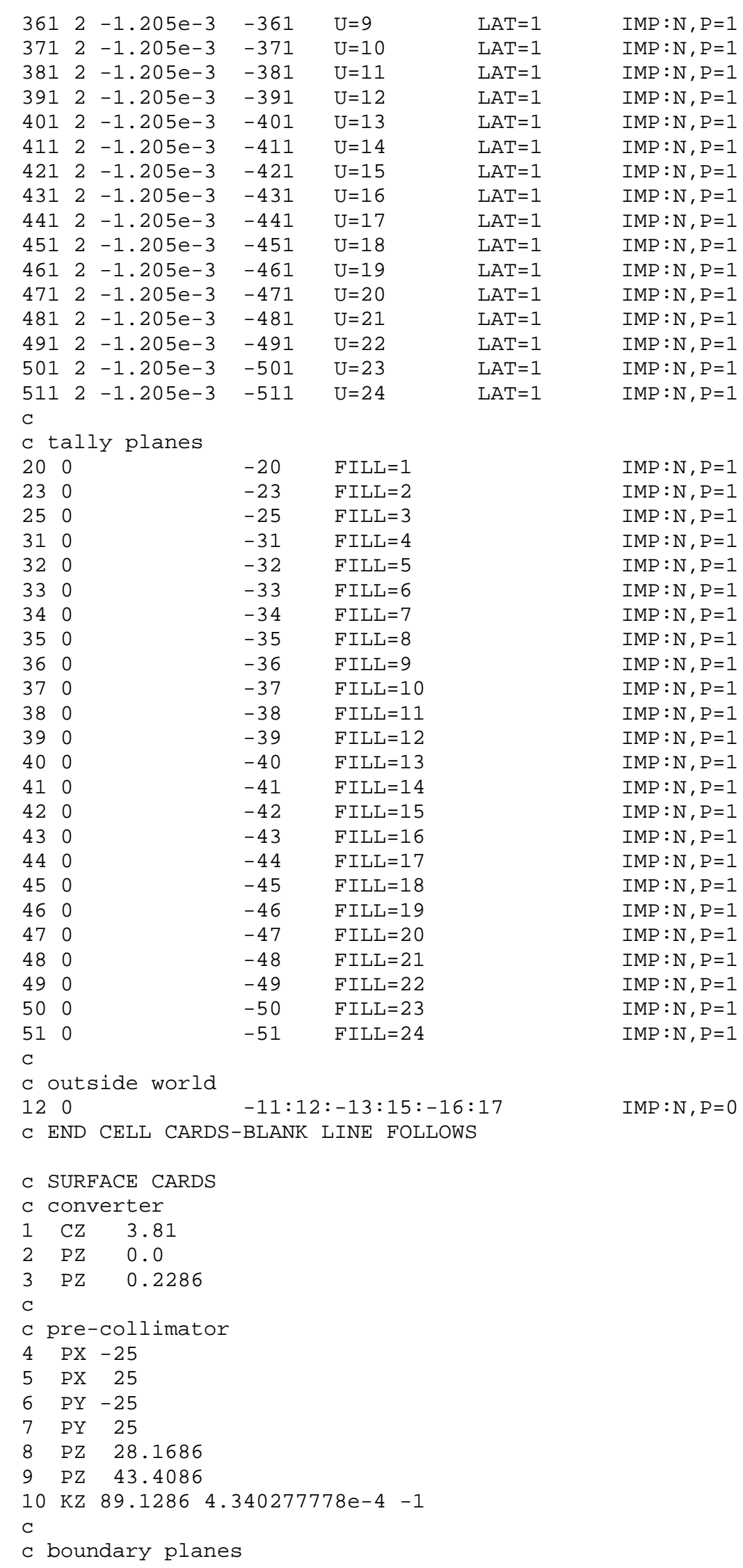




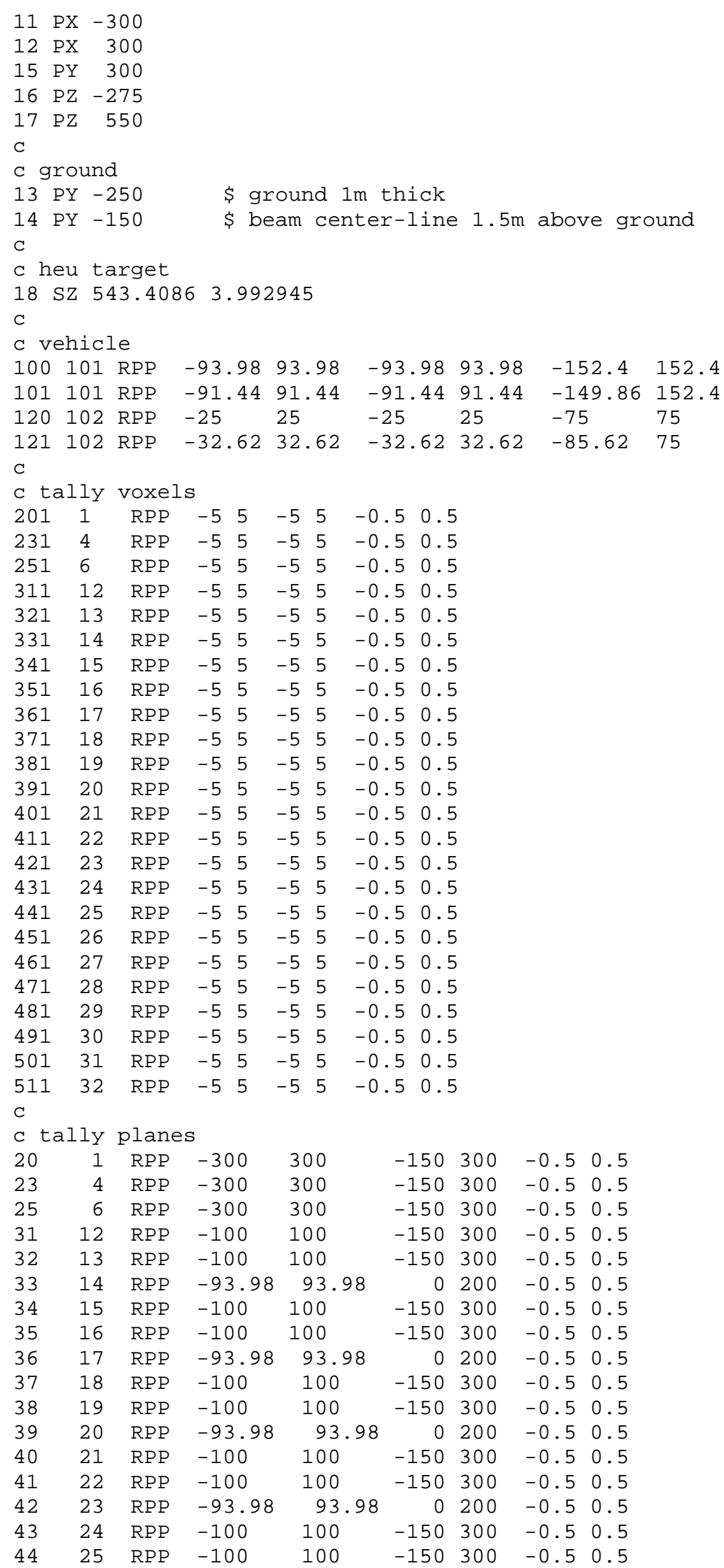




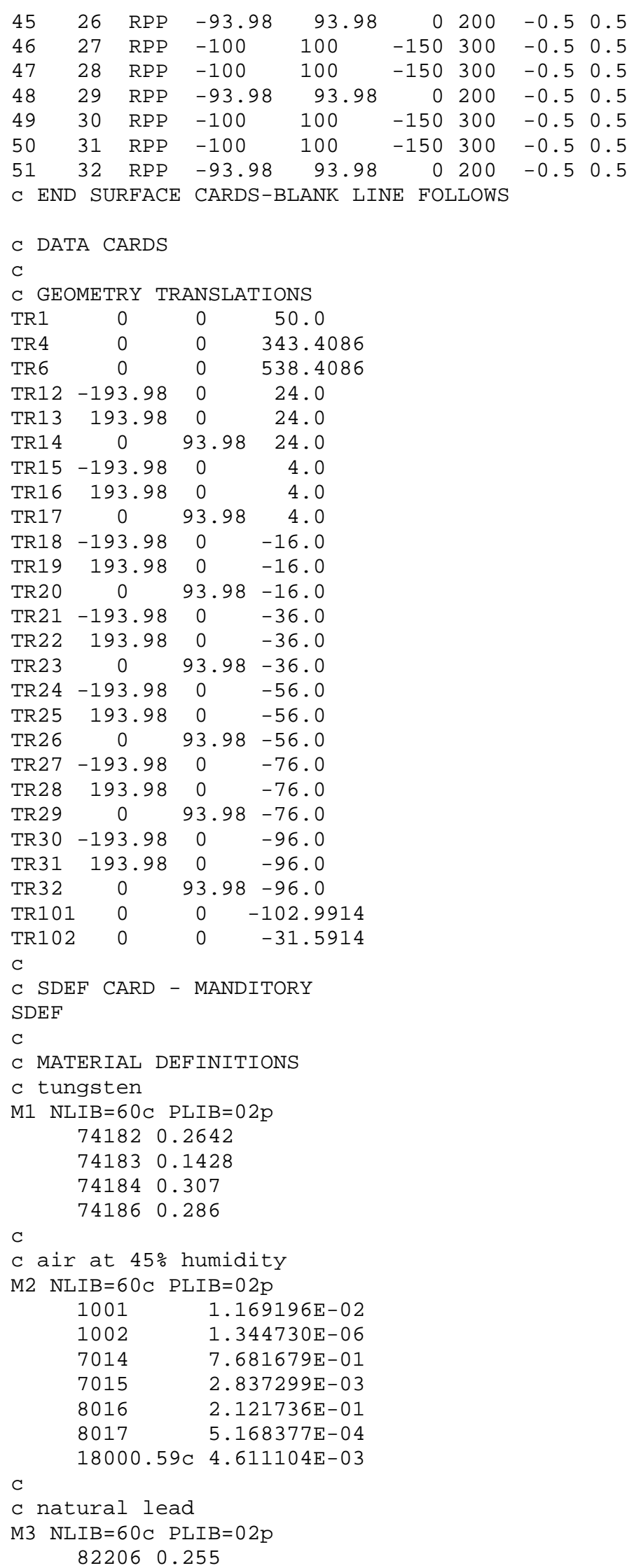




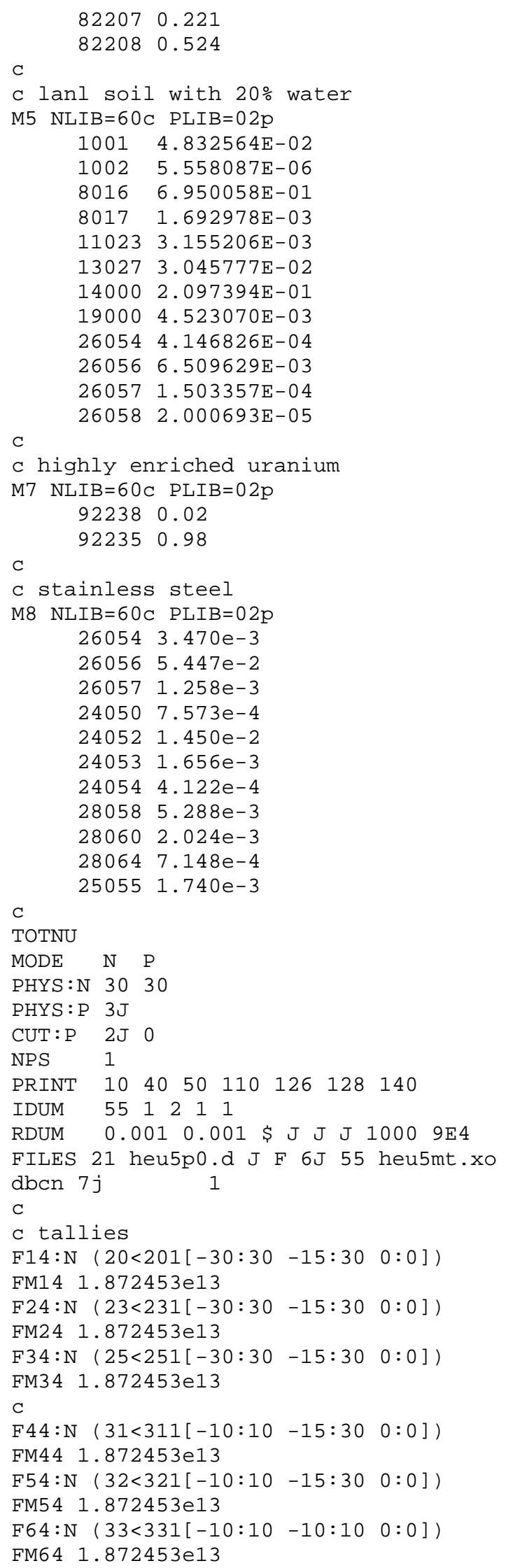




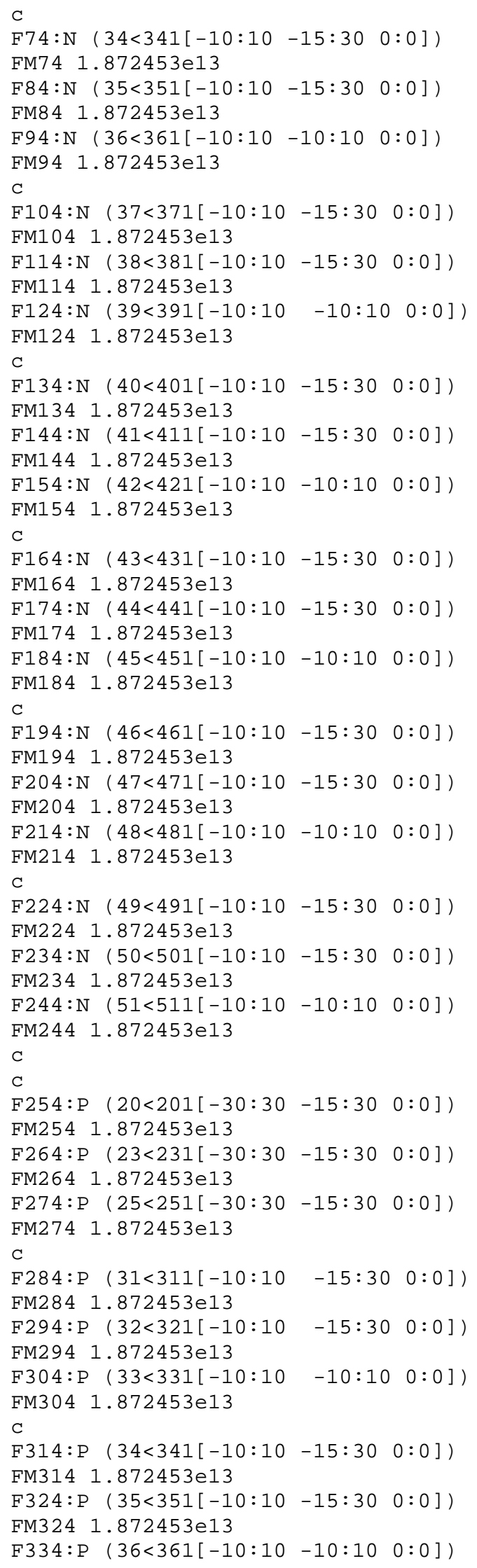


FM334 1.872453e13

$\mathrm{C}$

F344:P $(37<371[-10: 10 \quad-15: 30 \quad 0: 0])$

FM344 1.872453e13

F354:P $(38<381[-10: 10-15: 30 \quad 0: 0])$

FM354 1.872453e13

F364:P $(39<391[-10: 10 \quad-10: 10 \quad 0: 0])$

FM364 $1.872453 \mathrm{e} 13$

$\mathrm{C}$

F374:P $(40<401[-10: 10 \quad-15: 30 \quad 0: 0])$

FM374 1.872453e13

F384:P $(41<411[-10: 10 \quad-15: 30 \quad 0: 0])$

FM384 1.872453e13

F394:P $(42<421[-10: 10 \quad-10: 10 \quad 0: 0])$

FM394 1.872453e13

$\mathrm{C}$

F404:P $(43<431[-10: 10-15: 30 \quad 0: \odot])$

FM404 1.872453e13

F414:P $\left(44<441\left[\begin{array}{llll}-10: 10 & -15: 30 & 0: 0\end{array}\right)\right.$

FM414 1.872453e13

F424:P $(45<451[-10: 10-10: 10 \quad 0: 0])$

FM424 $1.872453 \mathrm{e} 13$

C

F434:P $(46<461[-10: 10 \quad-15: 30 \quad 0: 0])$

FM434 1.872453e13

F444:P $(47<471[-10: 10 \quad-15: 30 \quad 0: 0])$

FM444 1.872453e13

F454:P $(48<481[-10: 10 \quad-10: 10 \quad 0: 0])$

FM454 $1.872453 \mathrm{e} 13$

C

F464:P $(49<491[-10: 10 \quad-15: 30 \quad 0: 0])$

FM464 1.872453e13

F474:P $\left(50<501\left[\begin{array}{llll}-10: 10 & -15: 30 & 0: 0\end{array}\right)\right.$

FM474 1.872453e13

F484:P $(51<511[-10: 10 \quad-10: 10 \quad 0: 0])$

FM484 1.872453e13

C END OF FILE 

APPENDIX G

MCNPX INPUT FILE FOR THE DELAYED NEUTRON SOURCE 



\section{Appendix G. MCNPX Input File for the Delayed Neutron Source}

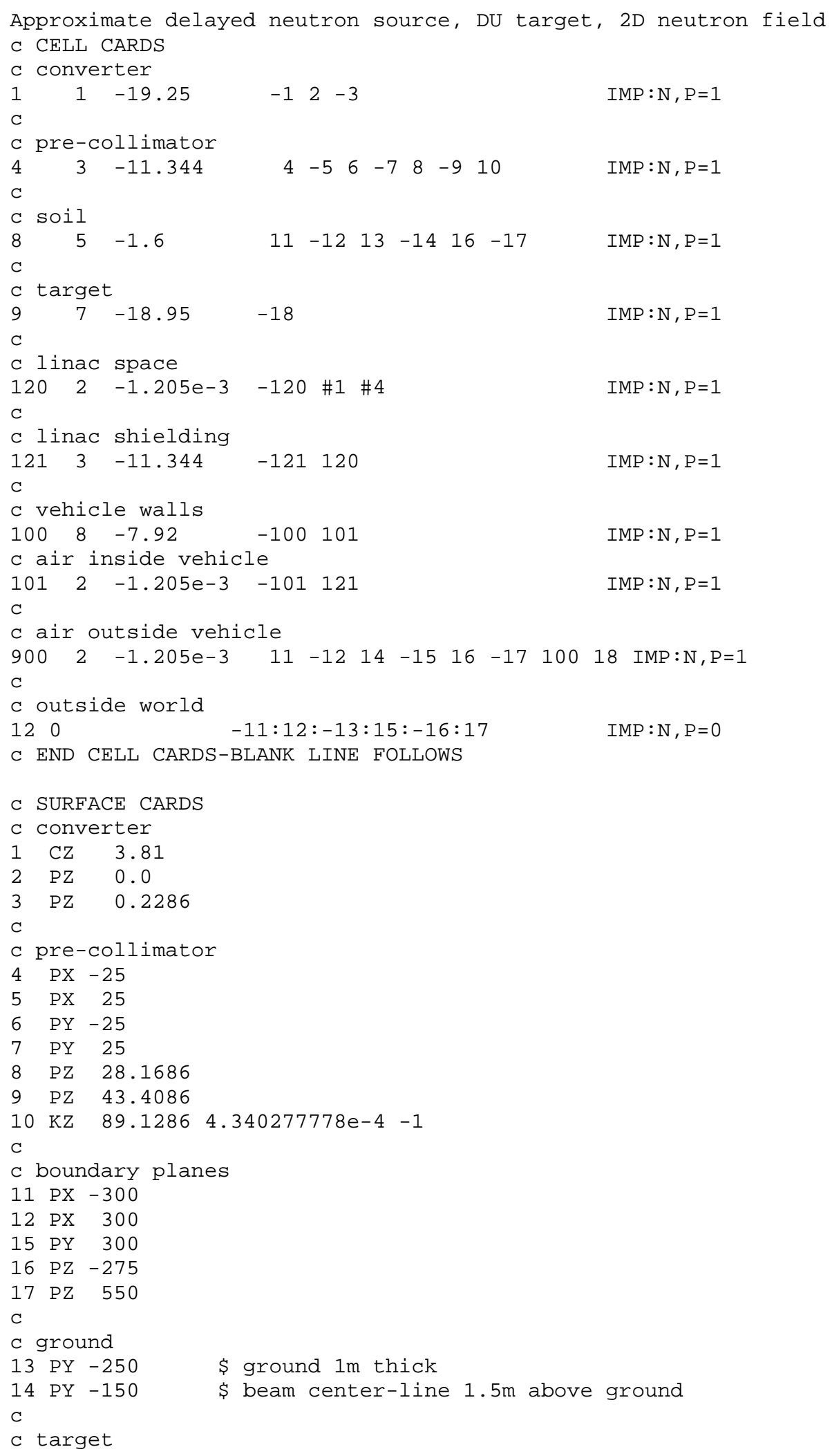




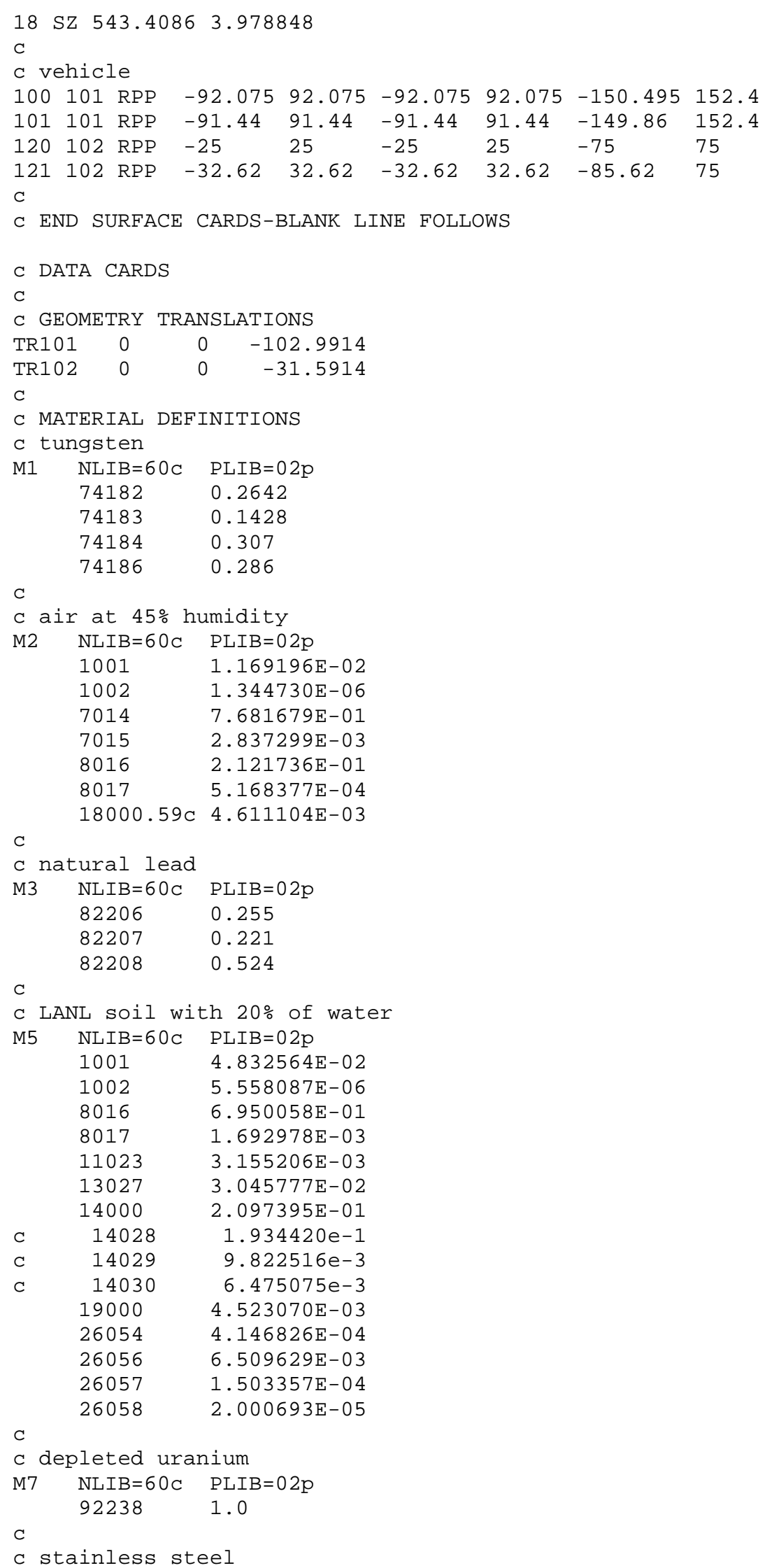




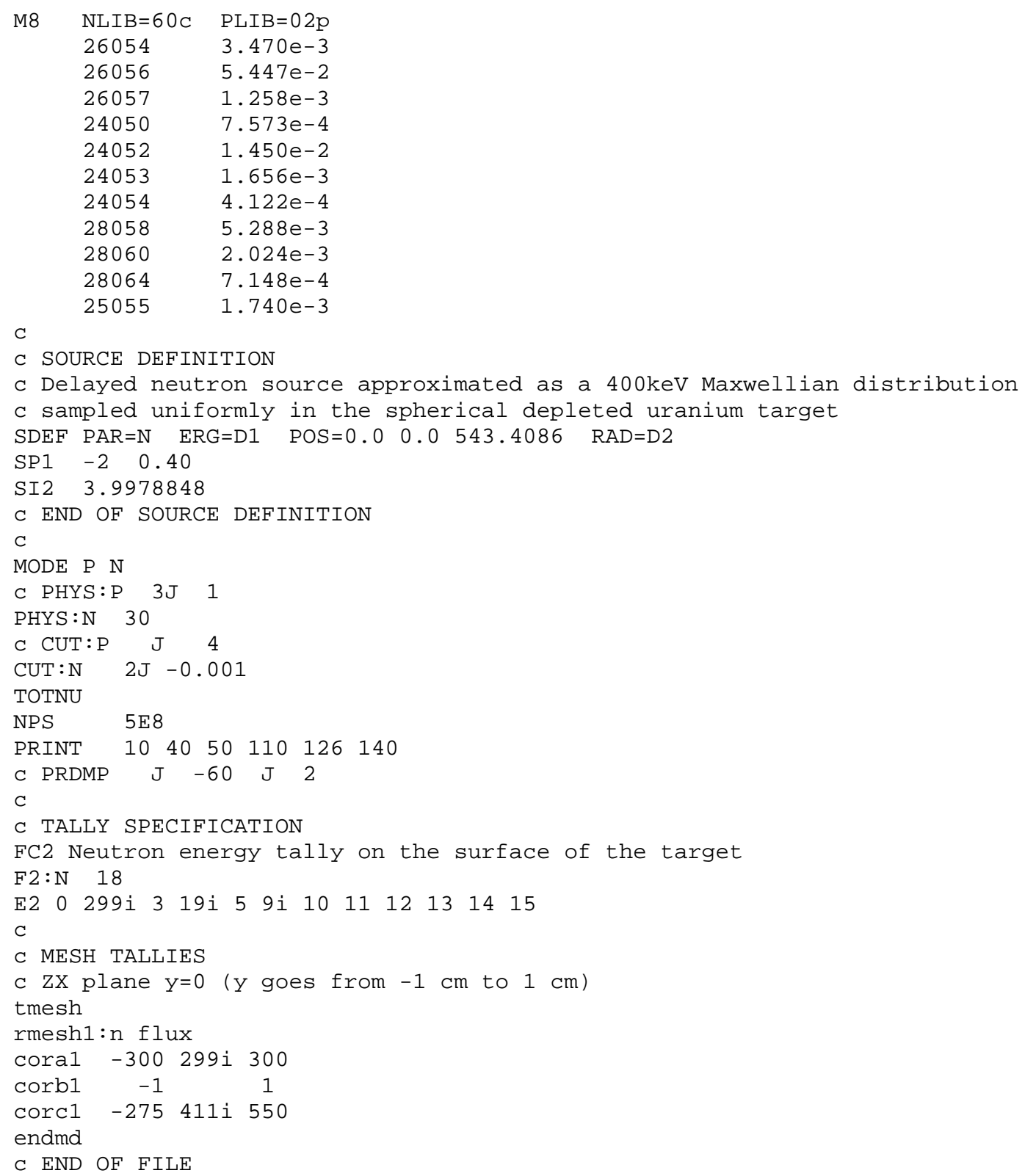



APPENDIX H

RELATIVE ERRORS ON DELAYED NEUTRON FLUENCE MAPS 

Appendix H. Relative Errors on Delayed Neutron Fluence Maps

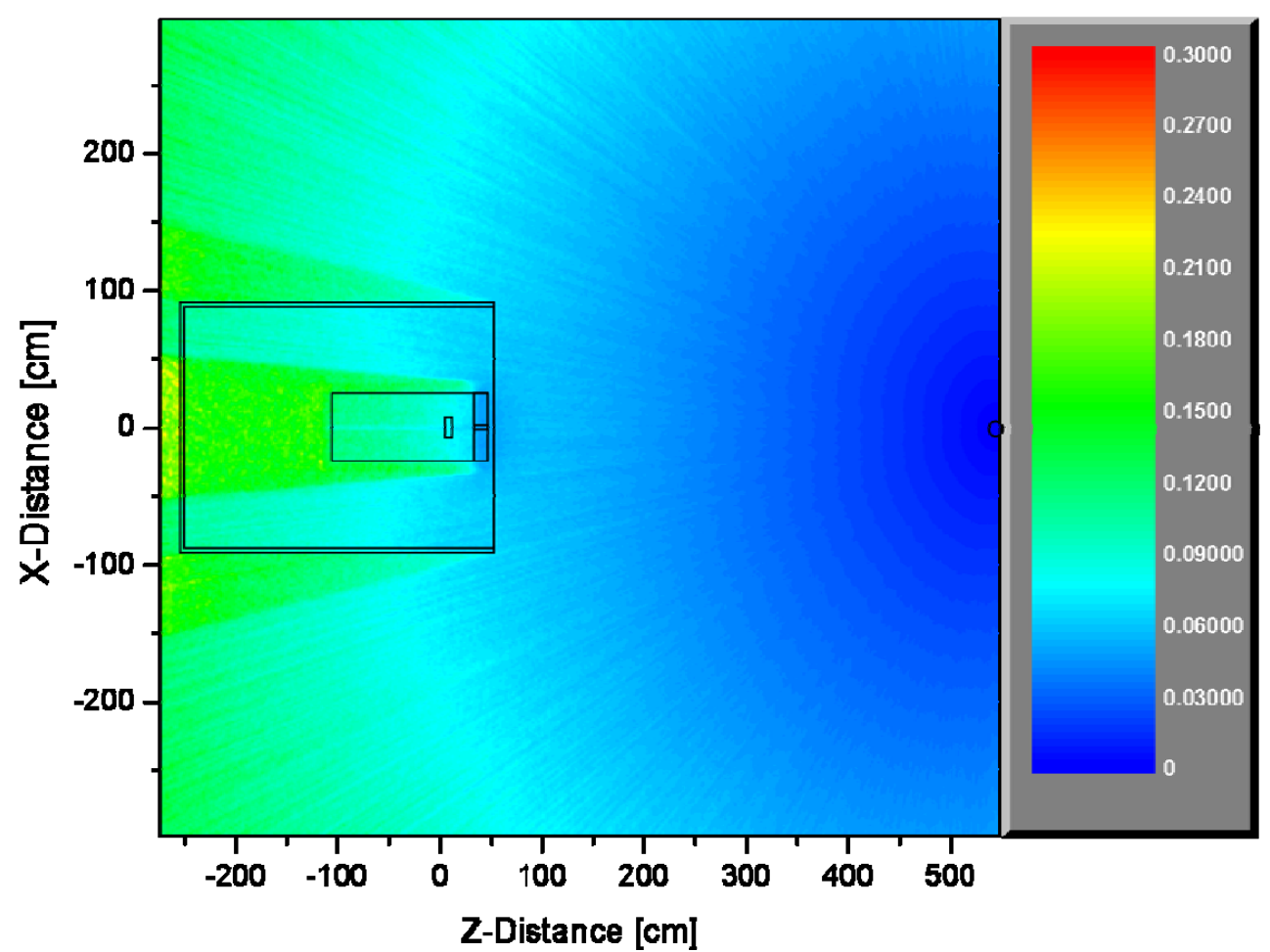

Figure H-1. Relative errors for the delayed neutron fluence map shown in Figure 51.

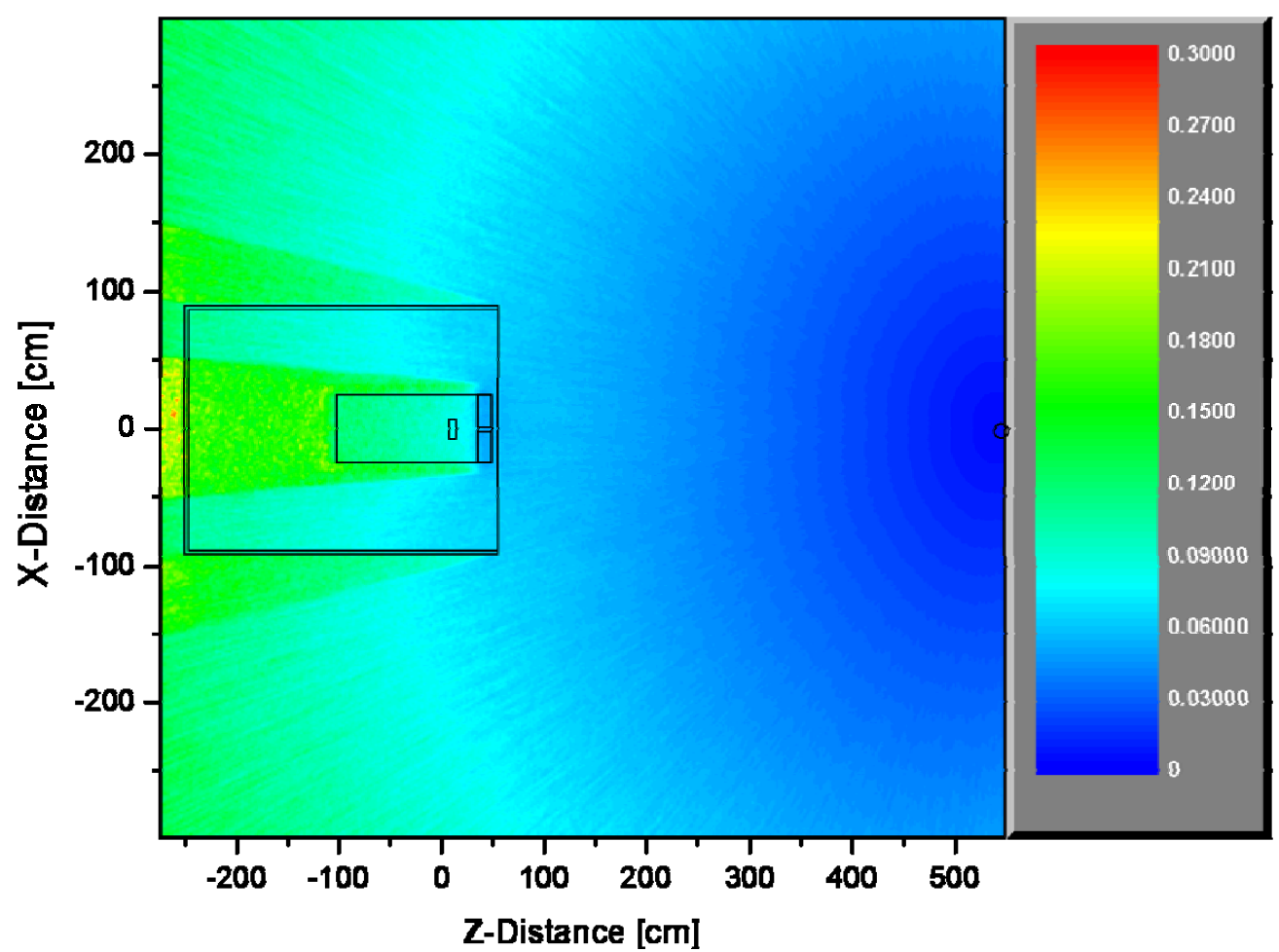

Figure H-2. Relative errors for the delayed neutron fluence map shown in Figure 52. 


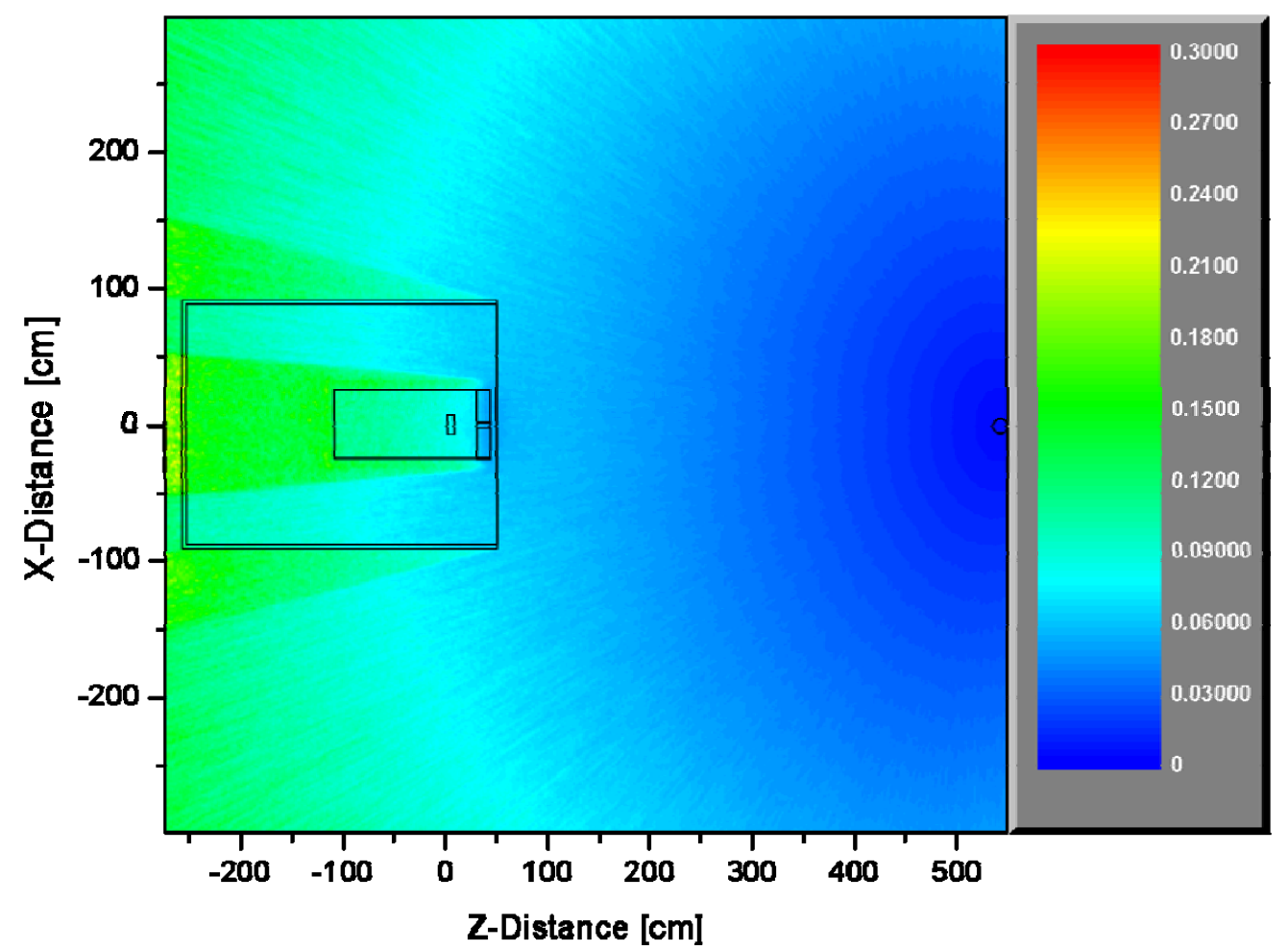

Figure H-3. Relative errors for the delayed neutron fluence map shown in Figure 53.

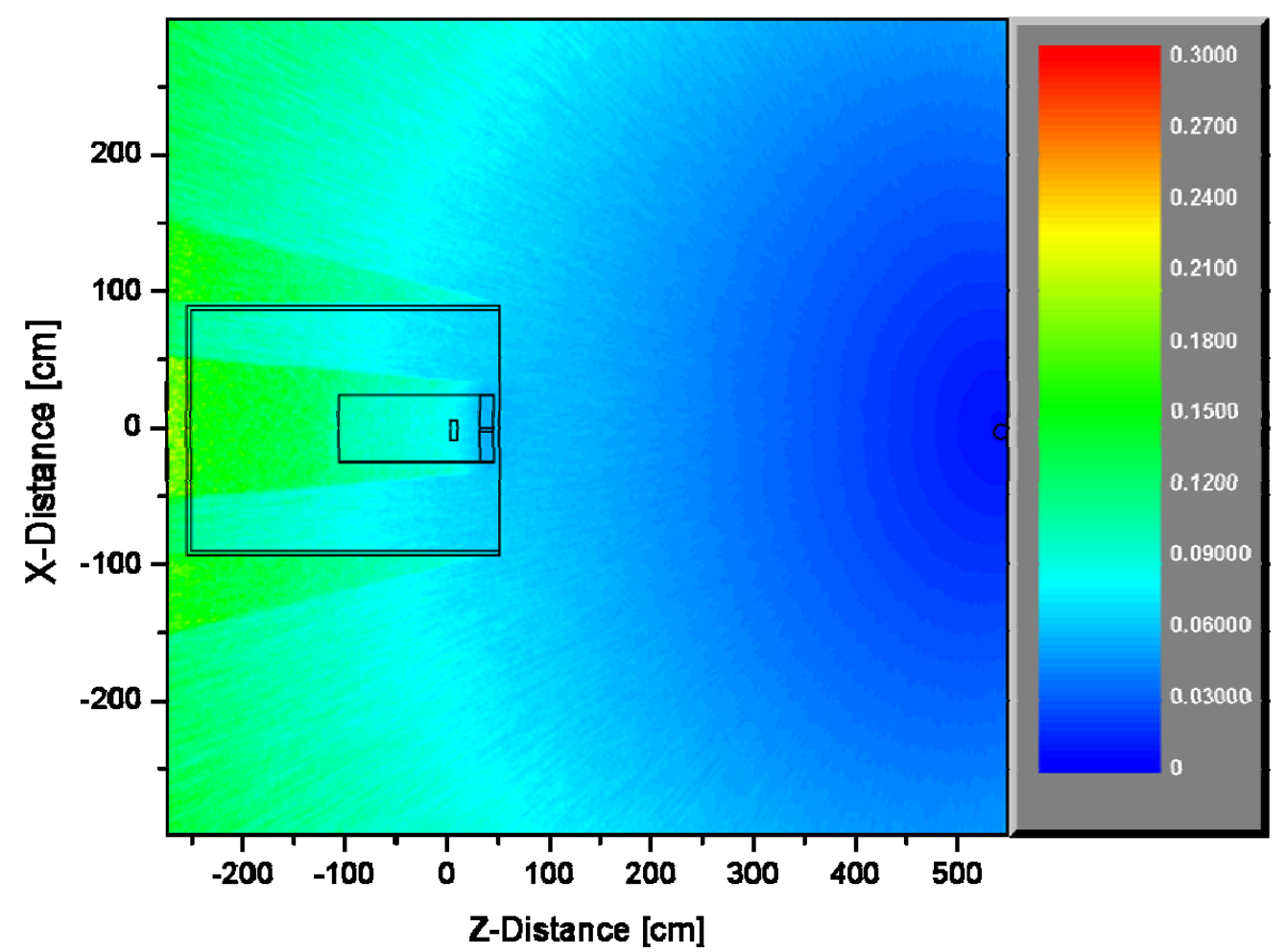

Figure H-4. Relative errors for the delayed neutron fluence map shown in Figure 54. 


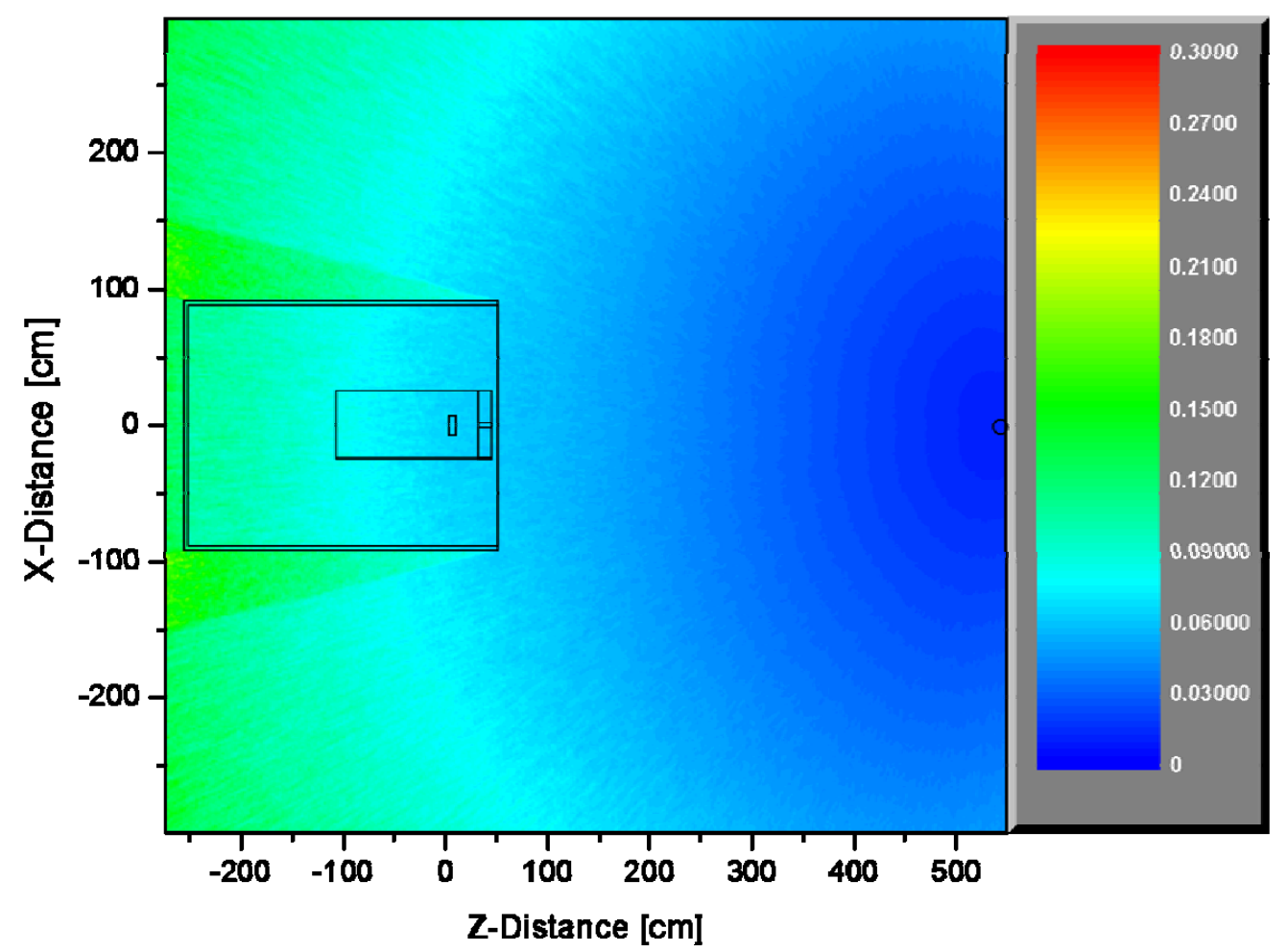

Figure H-5. Relative errors for the delayed neutron fluence map shown in Figure 55.

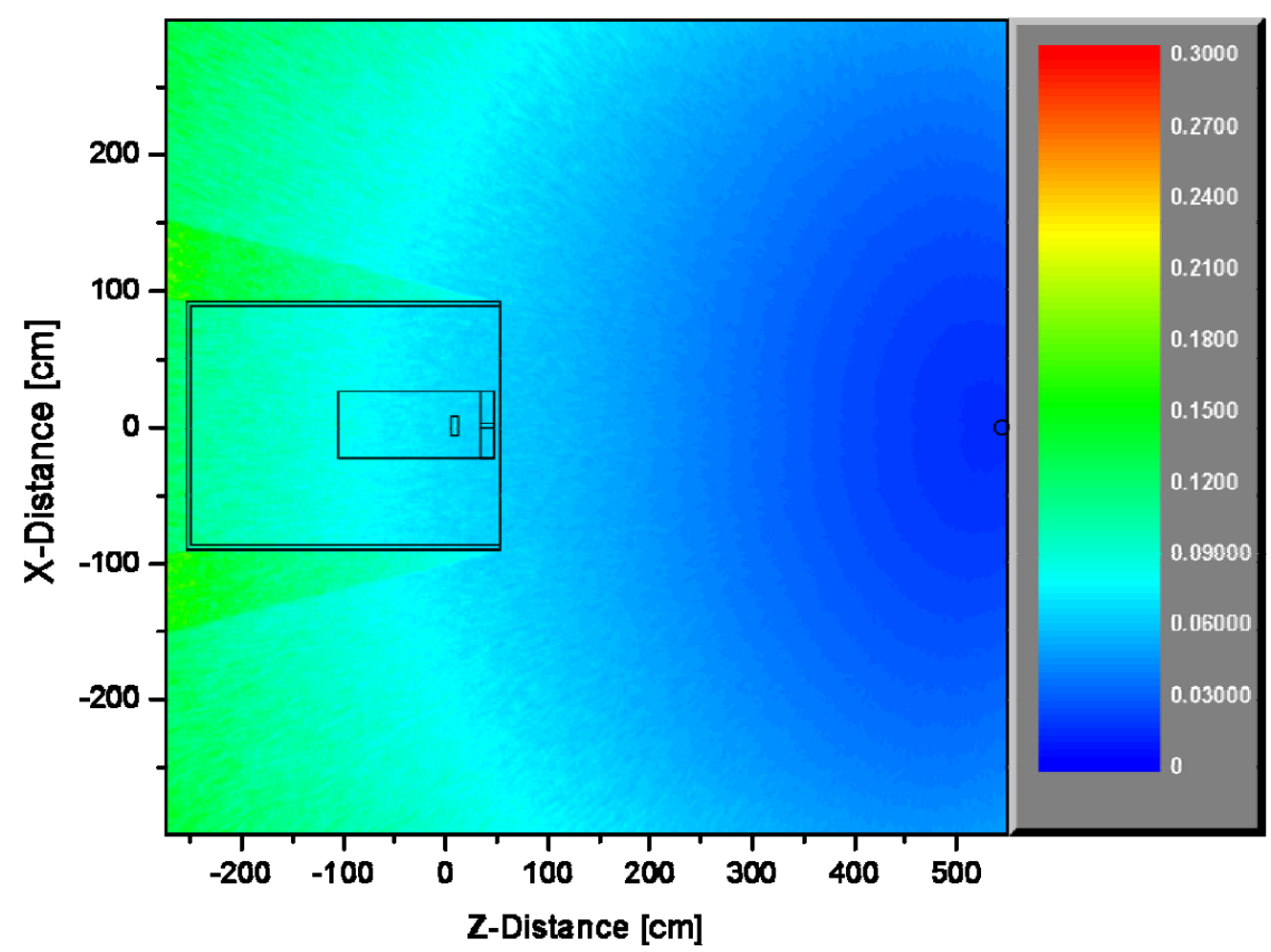

Figure H-6. Relative errors for the delayed neutron fluence map shown in Figure 56. 


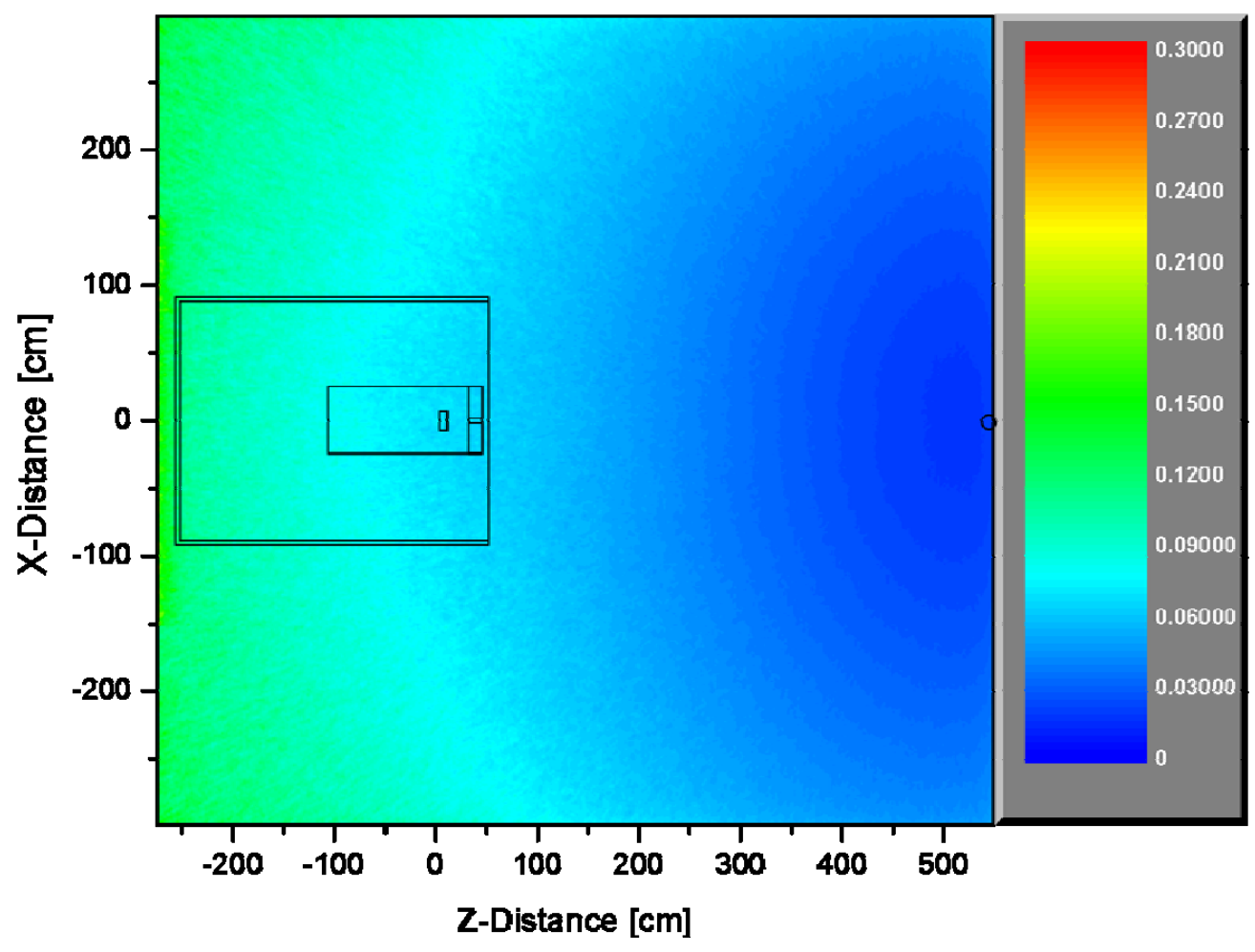

Figure H-7. Relative errors for the delayed neutron fluence map shown in Figure 57. 


\section{INTERNAL DISTRIBUTION}
1. Sara Pozzi
2. Marek Flaska
3. Thomas Miller
4. Vladimir Protopopescu 

